\title{
Acoustics of ancient Greek and Roman theaters in use today
}

\section{Gade, Anders Christian; Angelakis, Konstantinos}

Published in:

Acoustical Society of America. Journal

Publication date:

2006

Document Version

Publisher's PDF, also known as Version of record

Link back to DTU Orbit

Citation (APA):

Gade, A. C., \& Angelakis, K. (2006). Acoustics of ancient Greek and Roman theaters in use today. Acoustical Society of America. Journal, 120(5), 3148-3148.

\section{General rights}

Copyright and moral rights for the publications made accessible in the public portal are retained by the authors and/or other copyright owners and it is a condition of accessing publications that users recognise and abide by the legal requirements associated with these rights.

- Users may download and print one copy of any publication from the public portal for the purpose of private study or research.

- You may not further distribute the material or use it for any profit-making activity or commercial gain

- You may freely distribute the URL identifying the publication in the public portal

If you believe that this document breaches copyright please contact us providing details, and we will remove access to the work immediately and investigate your claim. 


\title{
Session 3aAAa
}

\section{Architectural Acoustics, Structural Acoustics and Vibration, Noise and Engineering Acoustics: Recent Developments in Acoustical Materials and Structures}

\author{
Brandon D. Tinianov, Cochair \\ Quiet Solution, 1250 Elko Dr., Sunnyvale, CA 94089-2213 \\ Kimihiro Sakagami, Cochair \\ Kobe Univ., Environmental Acoustics Lab., Faculty of Engineering, Rokkodai, Nada, Kobe 657-8501, Japan \\ Kirill V. Horoshenkov, Cochair \\ Univ. of Bradford, School of Engineering Design and Technology, Bradford, BD7 1DP, U.K. \\ Chair's Introduction-7:30 \\ Invited Papers \\ $7: 35$ \\ 3aAAa1. Characterizing viscoelastic and anisotropic porous materials. Laurens Boeckx, Poonam Khurana, Gerrit Vermeir, \\ Walter Lauriks (Laboratorium voor Akoestiek en Thermische Fysica, Celestijnenlaan 200D, BE-3001, Leuven, Belgium, \\ laurens.boeckx@fys.kuleuven.be), and Wim Desmet (Katholieke Universiteit Leuven, BE-3001, Belgium) \\ A wide range of commercial applications (building acoustics, food industry, automotive industry) can be found for porous \\ materials. Accurate material characterization and modeling is vital for the use of these materials in multilayered systems due to \\ increasing demands in acoustic comfort, noise legislation, and quality control during production. There is, however, a distressing lack \\ in raw, accurate material data and measurement methods concerning the characterization of these materials. Measuring and modeling \\ methods for the characterization of these materials will be presented. The experimental technique for the determination of the elastic \\ properties is based upon the excitation of waveguides in porous materials. The other parameters of the Biot-Allard model are predicted \\ using ultrasound. Acoustical properties can be predicted by using the measured structural material properties and incorporating them \\ into a transfer-matrix-based multilayered model.
}

3aAAa2. Linking microstructure and acoustic properties of open-cell foams. Camille Perrot, Raymond Panneton (GAUS, Dept. of Mech. Eng., Universite de Sherbrooke, QC, Canada, J1K 2R1, camille.perrot@ usherbrooke.ca), and Xavier Olny (ENTPE DGCB URA CRNS, Audin 69518, Vaulx en Velin, France)

A research program has been initiated in 2002 in order to link microstructure of high porosity open-cell foams to their acoustic properties. This paper is intended to highlight the main results of this study. The general objective of the research program is the determination of the acoustical macro-behavior from the physics at the local scale. A real rigid-framed porous media is studied. To this end, one needs first to determine the local geometry of the media, and second to solve over this geometry the partial differential equations that govern dissipation phenomena by thermal and viscous effects. The first step has been overcome by the technique of computed microtomography. This leads to experimental identification of the parameters of an idealized periodic unit-cell. The second step, solving harmonic heat and viscous fluid equations, is performed using Brownian motion and finite element simulations, respectively. Then, macroscopic behavior is obtained by spatial averaging of the local and frequency-dependent thermal and velocity fields. Results are presented in terms of two dynamic characteristic functions (viscous and thermal permeabilities) compared to impedance tube measurements. This computational methodology may be seen as a first step to optimize the microstructure of foams from a bottom-up approach for better sound proofing.

\section{8:15}

3aAAa3. Interlaboratory experiments on the characterization of the acoustical and related nonacoustical properties of porous media. Kirill Horoshenkov, Amir Khan (Univ. of Bradford, Bradford, UK), Frank Sgard, Francois Xavier, BecoLuc Jaouen, Amlie Renault, Nesrine Amirouche (Ecole Nationale des Travaux, Lyon, France), Jorn Hubelt (Gesellschaft fr Akustikforschung Dresden mbH (AFD), Germany), Francesco Pompoli, Nicola Prodi, Paolo Bonfiglio (Universita di Ferrara, Ferrara, Italy), Walter Lauriks, Laurens Boeckx (Katholieke Universiteit Leuven, Belgium), Giulio Pispola, Francesco Asdrubali (Univ. of Perugia, Perugia, Italy), Noureddine Atalla, Celse Amdin (Univ. of Sherbrooke, Sherbrooke, Canada), K. Mark Gmerek, and Adam Weston (The Boeing Co., Chicago, IL)

A series of reproducibility experiments on the characterization of acoustical parameters of selected samples of porous media is carried out on a range of porous samples in several independent laboratories in Europe and North America. The data on the characteristic acoustic impedance and complex propagation constant are presented in this work. In addition, the assessment of the related geometrical parameters required for modeling the acoustic performance of porous media, namely the steady-state flow resistivity, porosity, tortuosity, viscous and thermal characteristic lengths, and thermal permeability, is carried out. Detailed procedures related to sample preparation, and installation are discussed together with data on the material property variation observed between individual material samples and laboratories. 
3aAAa4. Sound absorption characteristics of a honeycomb-backed microperforated panel (MPP) absorber. Kimihiro Sakagami, Kosuke Nakajima, Masayuki Morimoto (Environ. Acoust. Lab., Faculty of Eng., Kobe Univ., Rokkodai, Nada, Kobe, 657-8501, Japan), Motoki Yairi, and Atsuo Minemura (Kajima Corp., Chofu, Tokyo, 182-0036, Japan)

Microperforated panels (MPPs) are typically made of a thin metal or plastic panel and are often unsuitable for an interior finish because thin limp panels do not have enough strength. In particular, an interior finish of room walls requires appropriate strength. In order to solve this problem, a honeycomb structure is attached behind MPPs to stiffen the construction. Thus, it is possible to stiffen an MPP without increasing its thickness, which is important to keep MPPs at their best absorption performance. Furthermore, a honeycomb can increase MPPs' absorption coefficient in a similar way as a porous layer backed by a honeycomb. In this study, an experiment was performed to gain insight into the acoustical effect of a honeycomb structure behind MPPs and a simple theoretical model to interpret the experimental effects is presented. The experimental results show that the honeycomb affects the absorption characteristics of MPPs: the absorption peak increases and shifts to lower frequencies. This effect becomes more significant as the thickness of the honeycomb increases. The results from the theoretical model show the same tendency. This is attributed to the fact that the honeycomb makes a similar condition to local reaction in the back cavity.

\section{8:55}

3aAAa5. Development of new sound insulators with perforated board and honeycomb layer systems. Masahiro Toyoda and Daiji Takahashi (U. and E. Eng., Grad. School of Eng., Kyoto Univ., Katsura C1-4-392, Nishigyo-ku, Kyoto 615-8540, Japan, ae.toyoda@archi.kyoto-u.ac.jp)

Two newly developed types of sound insulators are introduced in this study. The first type is derived from an analytical model of a vibrating surface with an impedance facing. The model is investigated theoretically; the results indicate the possibility of reducing radiation from the vibrating surface by giving appropriate impedance. To realize this effect, a model using a perforated board with a subdivided air cavity is proposed. It is shown theoretically and experimentally that this insulator can achieve radiation reduction at an arbitrary frequency. The second type is proposed from the viewpoint of the subdivision strategy. It presents the possibility of improving insulation by restricting the air-particle motion at the interface between the vibrating surface and air. This method for noise control has an attractive simplicity and considerable practical benefit. The attenuation mechanism is discussed theoretically and experimentally. Results of this investigation show that the effect of this insulator is characterized by reduction of acoustic radiation at low frequencies near and below the critical frequency of coincidence. Wide use of these two insulators in many fields is anticipated.

\section{9:15}

3aAAa6. The effects of damped panels on sound transmission loss through lightweight framed construction. Brandon Tinianov (Quiet Solution, 1250 Elko Dr., Sunnyvale, CA 94089)

The incorporation of damping into the design of materials has been long understood as an effective method for improving the transmission loss of undamped, lightweight structures. Classic examples of its application include marine vessels, aircraft, and ground transportation such as trains, trucks, and buses. However, damping has rarely been incorporated into the materials used for the lightweight construction techniques found in modern buildings. This paper will review the basic principles of designing improved damping into construction materials using the well-known RKU model. The presentation will also review the mechanisms of energy transfer through lightweight building systems and present the improvements in acoustical performance due to damped materials. Typical gains are from 3-18 points in single number ratings (STC, Rw) and greater than $20 \mathrm{~dB}$ in some $1 / 3$-octave bands. Extensive laboratory transmission loss data will be presented.

\section{Contributed Papers}

9:35

3aAAa7. Resilient ceiling construction in residential buildings. Anthony Nash (Charles M. Salter Assoc., 130 Sutter St., San Francisco, CA 94104, anthony.nash@cmsalter.com)

In multifamily buildings, the floor/ceiling assembly between living units needs to be sound rated. In North America, a common light-frame floor construction involves wood or cold-formed metal joists spaced at 400 $\mathrm{mm}$. The typical practice is to attach a gypsum board ceiling to the underside of the floor framing using narrow strips of "Z"-shaped thin metal called "resilient channels." If the resilient channels or the ceiling are installed incorrectly, the low-frequency sound insulation of the assembly can be seriously degraded. Based on our experience with resilient ceiling construction, there are two significant factors that have not yet been documented in an acoustical laboratory. One factor is to maintain a small separation around the entire perimeter of the ceiling where it intersects vertical wall surfaces (this void is later sealed airtight with a flexible caulking material). A second is the particular design of the Z-shaped resilient channel. The paper will discuss findings from two acoustical test series of floor/ceiling assemblies in wood-frame buildings. In both cases, the assembly was tested before and after modifications to the perimeter of the existing resilient ceiling. Each of the series also included tests of a complete ceiling reconstruction using an improved resilient channel design.
9:50

3aAAa8. The effect of damping materials on heavy-weight floor impact in apartment buildings. Jin Yong Jeon, Seung Yup Yoo, and Pyoung Jik Lee (School of Architectural Eng., Hanyang Univ., Seoul 133-791, Korea, jyjeon@hanyang.ac.kr)

Floating floors, in which resilient isolators are inserted between the structural slab and the upper layer of the floor, are generally used because of their effectiveness in controlling structure-borne and airborne noise. However, these isolators amplify low-frequency noises below $100 \mathrm{~Hz}$ that are generally produced by heavyweight impacts. Viscoelastic damping materials are widely used to reduce noise in settings such as vehicles, ships, and machinery; however, there has been no report of their use in apartment building structures for reducing floor impact sounds. The addition of damping can be embodied using viscoelastic liquid such as silicone gasoline; energy is absorbed with superior characteristics being a solid matter of viscoelastic materials. In this study, optimized thickness of the damping layer was investigated for the efficient reduction of low-frequency noise generated by heavy-weight floor impact. Effects of temperature variations on the characteristics of storage modulus and loss factor of the material were also investigated. 


\section{0:05-10:15 Break}

\section{0:15}

3aAAa9. Sound absorbing material made of polyester nonwovens. Shin-ichi Koga (Fukoku Co., Ltd., 254 Nakayama, Mitsuhashi-machi, Yanagawa, 832-0811 Japan), Kazutoshi Fujimoto, and Ken Anai (Kyushu Univ., Hakozaki, Higashi-ku, Fukuoka 812-8581 Japan)

Polyester nonwovens (PW) comprise fine fibers, similarly to glass wool $(\mathrm{GW})$. Therefore, PW is expected to have high sound-absorption capabilities. In recent years, application of PW, an entirely recyclable material, as an absorbent material has attracted much attention from the viewpoints of resource recycling and waste reduction. In some cases, manufactured PW has been used as an interior material in an actual acoustic laboratory. Reportedly, however, PW has slightly inferior sound absorption capabilities compared to GW. For wider use as a sound-absorbing material, PW must have better sound absorption. In this study, sound absorptions of PW were measured for various fiber compositions. Specifications that engender high sound absorption capabilities were examined. Results revealed PW specifications that provide similar sound absorption as GW with similar thickness and density. The PW presented in this paper has both high sound absorption and good environmental properties. Therefore, it is useful as a sound absorbing material not only for architectural use, but also in various other fields. Finally, two examples are introduced of an actual anechoic chamber that was finished using PW.

\section{$10: 30$}

3aAAa10. Sound-absorbing material made of recycled polyester nonwovens using scrapped pieces. Kazutoshi Fujimoto, Ken Anai, Tatsurari Nakano (Faculty of Human-Environment Studies, Kyushu Univ., 6-10-1 Hakozaki, Higashi-ku, Fukuoka 812-8581 Japan), and Shin-ichi Koga (Fukoku Co., Ltd., Mitsuhashi-machi, Yanagawa, 832-0811 Japan)

The authors developed polyester nonwovens (PW) that have nearly equivalent sound absorption to glass wool with similar thickness and density. Unfortunately, scraps amounting to $20 \%$ of crude polyester are generated during PW manufacturing and cutting. They must be discarded as waste materials. Therefore, the authors contrived an original recycling technique to use such scrap pieces. Sound absorption characteristics of recycled materials manufactured using this method (RPW) are inferred to differ from those of PW because of their different fiber compositions. In this study, sound absorption of RPW is measured and the following points are examined experimentally: the relationship between manufacturing processes and sound absorption of RPW; sound absorption of RPW when two kinds of scrap pieces are mixed; and effects of mixing crude materials on RPW sound absorption. Results indicate that RPW has sufficient sound absorption for practical use. Comprising scrapped pieces that have been discarded as waste, RPW addresses the social importance of good resources usage and waste reduction; it also offers advantages of lower cost than PW. This RPW material is anticipated for use in various fields as a sound-absorbing material.

\section{0:45}

3aAAa11. Propagation characteristics of airborne ultrasonic wave in porous materials. Kenichi Aoki and Tomoo Kamakura (The Univ. of Electro-Commun., 1-5-1 Chofugaoka, Chofu-shi, Tokyo 182-8585, Japan, aoki@ee.uec.ac.jp)

The attenuation coefficient and propagation speed of airborne ultrasound are measured for highly porous open-cell polyurethane foams and fibers at frequencies of $1 \mathrm{kHz}$ to $1.7 \mathrm{MHz}$. A theoretical model is proposed to explain the frequency responses of the transmission loss and speed of an air coupled wave physically in porous materials. The model is derived from Biot's flow resistance and density [M. A. Biot, J. Acoust. Soc. Am. 28, 179-191 (1956)], Lambert's bulk modulus of fluids in pores [R. F. Lambert, J. Acoust. Soc. Am. 72, 879-887 (1982)], and Zwikker and Kosten's concept for compliance of the side holes with entrance resistance [Sound Absorbing Materials (Elsevier, Amsterdam, 1949)]. Using measured data of static flow resistance to determine the mean pore size and the proposed model, theoretical prediction is made for the transmission losses and sound speeds in porous materials. Good agreement of theory and results of experiments in the whole frequency range supports the usefulness of the present model. Additionally, the model provides some findings for the extra attenuation coefficient of a slow wave in cemented glass bead specimens and sandstone for high-frequency ranges [P. B. Nagy, J. Acoust. Soc. Am. 93, 3224-3234 (1993)]

\section{1:00}

3aAAa12. Comparative results of field impact isolation testing of numerous resilient underlayment systems for hard-surfaced flooring. Marlund Hale (Advance Eng. Acoust., 663 Bristol Ave., Simi Valley, CA 93065, mehale@aol.com)

There is a strong ongoing interest in replacing carpet and pad flooring in existing multi-family residences and during apartment conversions to condominiums with hard surface flooring. Installing such flooring often results in a significant decrease in the impact isolation performance of the floor/ceiling assembly. Some municipalities and/or homeowners associations have enacted regulations requiring that floor/ceiling assemblies meet specific minimum laboratory and/or field acoustical performance standards. This paper presents published vendor data and field performance test results for two condominiums in a multi-family residential complex for which both the city and the homeowners association have such performance requirements. A series of field tests was undertaken to assist the homeowners in selecting qualifying underlayment systems for their preferred hard surface flooring replacement of their existing quality carpet and pad flooring. The test flooring systems were each installed by a flooring contractor. All aspects of these field tests were maintained as identical as possible, with the only variable being the different commercial resilient underlayment products. Published vendor data of certified laboratory IIC test results are compared with the FIIC field test results.

\section{$11: 15$}

3aAAa13. Reflectivity of absorptive surfaces. Jonathan Rathsam and Peter D'Antonio (RPG Diffusor Systems, Inc., 651-C Commerce Dr., Upper Marlboro, MD 20774)

The random incidence absorption coefficient is a very coarse metric. In many small and large room applications, a directional absorption coefficient more accurately describes potential problems from first-bounce specular reflections. An impedance tube is useful for evaluating normal incidence absorption, but a more general approach to determining a material's specular reflectivity or first-bound attenuation is needed. The AES4id-2001 information document describes such a method. This study compares the "first-bounce specular attenuation" of $1 \mathrm{ft}(\mathrm{H}) \times 3 \mathrm{ft}(\mathrm{W})$ and 4 $\mathrm{ft}(\mathrm{H}) \times 8 \mathrm{ft}(\mathrm{W})$ samples of 2-in.-thick, 6 pcf fiberglass absorbers (with and without fabric covering) with that of a rigid reflector, using a $2 \mathrm{D}$ boundary method goniometer. The boundary wave's arrival is calculated and identified in the impulse response measurements. The use of small and large samples allows evaluation of both the fabric covering and boundary wave effects on the specular attenuation, for normal and 45-deg incidence. Time and frequency domain results are presented. 


\title{
Session $3 \mathbf{a A A b}$
}

\section{Architectural Acoustics: Acoustics of Large Indoor and Outdoor Performance Spaces II}

\author{
Daniel R. Raichel, Cochair \\ 2727 Moore Ln., Fort Collins, CO 80526 \\ Toshiki Hanyu, Cochair \\ Nihon Univ., Narashinodai, Funabashi, Chiba 274-8501, Japan
}

Contributed Papers

8:00

3aAAb1. Acoustics of ancient Greek and Roman theaters in use today. Anders Christian Gade and Konstantinos Angelakis (Acoust. Technol., Tech. Univ. of Denmark, Bldg. 352, DK 2800 Lyngby, Denmark, acg@oersted.dtu.dk)

In the Mediteranan area a large number of open, ancient Greek and Roman theatres are still today facing a busy schedule of performances including both classical and contemporary works of dance, drama, concerts, and opera. During the EU funded "Erato" project and a subsequent master thesis project, extensive measurement data have been collected from three well-preserved theatres, which represent three different stages of enclosing the audience in an open-air environment: (1) the Epidaurus Theatre in Greece without skenae wall or columnade behind the cavea; (2) the Jerash South theatre in Jordan with skenae wall but no columnade; and (3) the Aspendos Theatre in Turkey having both a full skenae building and a columnade around the cavea. In the paper the acoustic characteristics of these theatres will be compared and discussed in the light of acoustic performance requirements for modern use.

\section{$8: 15$}

3aAAb2. Acoustical design issues for contemporary places of worship. Jason R. Duty and David R. Schwind (Charles M. Salter Assoc., Inc., 130 Sutter St., Ste. 500, San Francisco, CA 94104, jason.duty@cmsalter.com)

This presentation intends to discuss the acoustical design of contemporary places of worship in the range of 500 to 3000 seats. The wide variety of programming requirements, concurrent uses, space constraints, and undesirable adjacencies in contemporary churches can be a similar challenge to many performing arts spaces. Many contemporary churches have loud, amplified music in their services and children's classrooms. In addition, religious study courses in adjacent classrooms are being held at the same time as the main worship service. During off-hours the spaces are used concurrently for teaching, conferences, weddings, prayer, and rehearsals. In many cases, the rooms outside of the main worship space are undersized and have undesirable adjacencies to each other as well as the main worship space. Using case studies we will discuss sound isolation design to reduce interference between concurrent unrelated uses, as well as room shaping and room finish treatments (including variable acoustics) to address room acoustics and the large sound systems.
$8: 30$

3aAAb3. Acoustical design of Sejong Performing Arts Center recital hall. Jin Yong Jeon and Yong Hee Kim (School of Architectural Eng., Hanyang Univ., Seoul 133-791, Korea, jyjeon@hanyang.ac.kr)

Acoustical design of a convention center into a recital hall of Sejong Performing Arts Center located in Seoul, Korea was carried out to renovate its function. The area of the convention space is about $612 \mathrm{~m}^{2}$ and it is mainly used for meetings, symposia, and so on. The volume of the existing hall is limited to $3100 \mathrm{~m}^{3}$, which causes lack of late reflections, due to its structural limitations. Therefore, the existing ceiling slab was designed to be pulled up so that a new ceiling can create extra volume of $1000 \mathrm{~m}^{3}$. A leaf-shaped floor plan and tilted side balconies within 16-m width between side walls were designed to adequately increase enough lateral reflection and spatial impression. The inclined side wall of 1:10 slope was proposed through the comparison with different angles of walls. The saw-tooth-shaped GFRC (glass fiber reinforced concrete) wall reflectors and center protruded stage rear wall are designed for sound diffusion. From the results of computer and scale modeling, it was predicted that the new space will have adequate reverberation time of $1.5 \mathrm{~s}$ and a uniform distribution of sound-pressure levels within $3 \mathrm{~dB}$.

\section{$8: 45$}

3aAAb4. A study on the influence of geometrical characters to the acoustical performance in vineyard terraced concert halls. Yi-run Chen and Wei-hwa Chiang (Dept. of Architecture, Natl. Taiwan Univ. of Sci. and Technol., \#43, Sec.4, Keelung Rd., Taipei, 106, Taiwan, d9413002@mail.ntust.edu.tw)

There are two distinctive features defining vineyard terraced concert hall: audiences surrounding the stage and vineyardlike seating arrangement. Besides that, people may find more differences than similarities within those limited built cases. This paper investigated the influence of geometrical characters to acoustical performance in vineyard halls using computer simulation. Through case studies of nine vineyard terraced halls, some geometrical characters were selected as research variables, namely volume, overall proportion, dimensions of key elements, seating capacity, arrangement of vineyard terraces, terraced side box shaping, and ceiling shaping. Acoustical parameters EDT, LF, C80, and G were analyzed by varying architectural features of a prototype developed based on the main geometrical characters of the nine halls. It has been found that raising terraced side boxes would help increase G and LF. For a constant room volume, increasing the number of terraces with sufficient height would raise the LF value in the nearby area mostly. Changing ceiling shape only had influence on the rear part of the front terrace. 
3aAAb5. Alternatives to the traditional concert hall experience. Bobby GibbsII, Jonas Braasch, and Ted Krueger (Rensselaer Polytechnic Inst., 110 8th St., Troy, NY 12180)

As relates to live performance, the purview of sound quality in architectural acoustics is mostly limited to concert halls. While the concert hall is perhaps the most common setting in which to hear live Western classical music in our present day, this was not always the case historically. As will be shown from analysis of published text as well as interviews with music critics, artists, and concert-goers, a variety of alternatives to the concert hall exists currently in the form of domestic settings, casual settings like coffee shops, as well as large outdoor settings like farms and parks. The advantages and disadvantages of such alternative venues, as concerns the overall concert experience and the quality metric of intimacy particularly, will be discussed. Moreover, design issues relating to the acoustical environment as well as the social environment in these venues will be discussed based on the data. This paper will ideally foster discussion in the acoustical and the architectural community on ways to expand the traditional concert hall experience as well as address design issues in alternative venues. [Work supported by ASA Minority Fellowship and RPI Humanities, Arts, Architecture, and Social Sciences Fellowship.]
3aAAb6. New approach of the model experiment in room acousticsadvantage of $\frac{1}{\mathbf{1 6}}$ scale model. Hidemaro Shimoda (Shimizu Corp., 3-4-17 Etchujima, Koto-Ku, Tokyo, 135-8530 Japan) and Yasuhiko Tahara (Tohoku Bunka Gakuen Univ., Aoba-Ku, Sendai, 981-8551 Japan)

In the construction of the auditoria, the examination by the acoustic model experiment has been often performed. This paper describes the outline of the result of investigating various features of the method of the experiment by $\frac{1}{16}$ scale models compared with models of $\frac{1}{10}$ and $\frac{1}{20}$ scales that have been generally adopted up to now. That is, the following advantages are found in the case study of the $\frac{1}{16}$ scale model experiment, and the new approach to the model experiment method is proposed here. (1) full cover of the band to $4 \mathrm{kHz}$ enables the quantification of a practical physical acoustic parameters. (2) The parameter as the air absorption coefficient in the supersonic wave band by $\frac{1}{10}$ scale models can be used. (3) Cheap digital sound recording system can be used considering the microphone characteristics. (4) The model construction cost can be largely saved compared with $\frac{1}{10}$ models.

THURSDAY MORNING, 30 NOVEMBER 2006

LANAI ROOM, 9:45 TO 11:30 A.M.

\title{
Session 3aAAc
}

\section{Architectural Acoustics: Acoustic Design Factors for Performing and Recording Spaces}

\author{
David L. Adams, Cochair \\ David L. Adams Associates, Inc., 1701 Boulder St., Denver, CO 80211 \\ Kanako Ueno, Cochair \\ Univ. of Tokyo, Inst. of Industrial Science and Technology, 4-6-1 Komaba, Meguroku, Tokyo 153-8505, Japan
}

Chair's Introduction-9:45

Contributed Papers

9:50

3aAAc1. Quantitative evaluation of characteristics of an auditorium shape in horizontal cross section. Kazuma Hoshi (Grad. School of Sci. and Technol., Nihon Univ., 1-8-14, Kandasurugadai, Chiyoda-ku, 101-8308, Tokyo, Japan, hoshi@arch.jcn.nihon-u.ac.jp), Toshiki Hanyu, and Katsuaki Sekiguchi (Nihon Univ., Chiyoda-ku, 101-8308, Tokyo, Japan)

The room shape of an auditorium is often classified as a "shoe-box type," "fan-shape type," "round type," "surrounding (vineyard) type," or other shape. The surface diffusivity index (SDI), which is an evaluation index of surface irregularities, has been proposed by Haan and Fricke, but the SDI is based on visual surveys of photographs or drawings [Appl. Acoust. 51(1), 53-69 (1997)]. Clarification of the relationship between an auditorium's shape and its acoustic characteristics would be simplified if the auditorium shape were understood objectively rather than subjectively. Therefore, in this study, we tried to objectively obtain characteristics of a room shape in horizontal cross section using an elliptic Fourier descriptor, which can detect the periodicity of a two-dimensional contour. Room shapes were expressed as spatial frequency characteristics calculated from their Fourier descriptors. Based on them, we can understand the amplitude of irregularities and their scale. In addition, by using principal component analysis, it is possible to understand, quantitatively, the main differences of room shapes in horizontal cross section.
10:05

3aAAc2. Design and construction of a small sound testing room in an office building. Edward C. Duncan and Kenneth Kaliski (Resource Systems Group, Inc. 55 Railroad Row, White River Junction, VT 05001)

A small sound-testing room has been constructed in the basement of an office building located within $50 \mathrm{ft}$. of railroad tracks. The room was designed to be multifunctional with both reverberant and semianechoic settings. The sound isolation of the room was designed with the expectation of conducting active noise reduction tests with sound-pressure levels of $120 \mathrm{~dB}$ and higher. The room is also being used to roughly estimate absorption coefficients of various materials. The room is $10.5 \times 9.75$ $\times 7.5 \mathrm{ft}$. with a volume of $768 \mathrm{ft}^{3}$. It is composed of a concrete floor and gypsum walls and ceiling. The walls are isolated from the floor with rubber isolation pads. The ceiling is constructed of 5/8-in. gypsum, 1/2-in. plywood, and a 2-in. layer of sand. The wall construction is $2 \times 4$ wood studs, two layers of 5/8-in. gypsum resiliently attached, and 3 in. of fiberglass. The entrance to the room is made of double solid core wood doors with a composite isolation and absorption layer in between both doors. Other design components, actual sound transmission testing results, reverberation times, modal issues, and uses for the room are discussed. 
of these modalities as critically linked and ultimately inseparable. Research investigating expected levels of reverberance given 2-D visual representations of several environments shows that perception of reverbera-

3aAAc3. Introduction of auditory filter into evaluation of characteristics of small enclosures. Saori Shimada, Hisaharu Suzuki, and Akira Omoto (Omoto Lab., Dept. of Acoust. Design, Faculty of Design, Kyushu Univ., Shiobaru 4-9-1, Minami, Fukuoka 815-8540, Japan)

Physical measures, which are useful for evaluating small enclosures such as studios and car cabins, are examined. Especially, auditory filtering using Zwicker's loudness model is introduced for calculating the measures. Most current measures used in evaluating the large sound field such as concert halls are based on measured or calculated impulse responses. Instead, the music signals of some genres are used in this study. Using the auditory model, the amount of information can be reduced, even when complicated signals are used as stimuli: the signals are convertible to information in 25-bark bands. Correspondence of such physical quantities and subjective judgments are considered from the viewpoint of sound-field similarity. Subjects are asked to judge the similarity of two successive sounds whose frequency characteristics are modified artificially using the equalizer. The analysis results obtained using the auditory filter were compared with the subjective evaluation. Results indicate that analysis using the auditory filter can yield fairly good correspondence with the subjective evaluation, especially in a middle frequency band. Regarding the lowfrequency band, the degree of correspondence depends on the musical genre. Expanding the effective frequency bands is an important theme of our ongoing research.

\section{0:35}

3aAAc4. Cross-modal perception of room acoustics. Anthony McCreery and Paul Calamia (Rensselaer Polytechnic Inst., 110 8th St., Troy, NY 12180, mccrea@rpi.edu)

The development of perceptually accurate audio-visual systems has been hindered by the fact that traditional scientific investigations into auditory and visual perception in humans have separated the two senses into separate and distinct modalities. It is important to consider the perception tion is heavily dependent on the visual appearance of a room. For the experiments to be discussed here, subjects were asked to adjust the level of reverberant sound that accompanied video of a person speaking in three different rooms: a library, a playhouse, and a cathedral. The results show that reverberance levels chosen by subjects rarely match those measured from impulse responses recorded in the rooms being presented. Experimental methodology and results as well as implications for the development of future audiovisual systems will be discussed.

\section{0:50}

3aAAc5. Considering source directivity in a recital hall with seats surrounding the platform. Wei Lin, Weihwa Chiang, and Yi-run Chen (Dept. of Architecture, Natl. Taiwan Univ. of Sci. and Technol., \#43, Sec.4, Keelung Rd., Taipei, 106, Taiwan, d9313001@mail.ntust.edu.tw)

The impact of source directivity can be significant for chamber music performances, in particular for arena-type halls that have visual intimacy. This study has been carried out to understand the physical and perceptual aspects of the seats surrounding the platform in recital halls. Effects of architectural features were evaluated using computer simulations that accounted for source directivity and diffusive reflections. Both live and recorded subjective evaluations of chamber music performances were made in an actual hall while acoustical measurements were also taken. As a result, computer simulations using a singer source showed significant decrease in both clarity and strength in the $2-\mathrm{kHz}$ band for the seats surrounding a centered platform. A wall $6 \mathrm{~m}$ in front of the platform was effective in mitigating the problems. Sound levels calculated from the recordings taken from various viewing angles were correlated with subjective evaluations, especially in the high frequencies. Timbre appeared to be the principal factor influencing solo performances and balance was the most important in judging ensembles. Nevertheless, live evaluation showed no significant difference among the listening positions.

\section{Contributed Poster Paper}

Poster paper 3aAAc6 will be on display from 8:00 a.m. to 11:30 a.m. The author will be at the poster from 11:05 a.m. to 11:30 a.m.

3aAAc6. Acoustic design of studio to record effect sounds in. Shinichi Oda, Junko Yokoyama, and Junichi Mikami (Dept. of Eng. Administration, Japan Broadcasting Corp. (NHK), 2-2-1 Jinnan, Shibuya-ku, Tokyo 150-8001, Japan)

We reconstructed a studio that has a space in which to record effect sounds. In this space, we record walking sounds on wooden floors, tile floors, sand, grass, and so on. This studio was planned on a floor with a light allowable load because the floor was formerly the roof of the building. A recording studio is usually constructed with a floating structure. The thin floating floor causes vibration of floorboards. A thick floor is heavy. In addition, we recorded the sound when we jumped on the floor. The noise from the structure disturbs the effect sounds that we need if the vibration caused by jumping stimulates the studio structure. For those reasons, we planned the following design to record pure effect sounds: The effect sound space is insulated from other spaces with the room structure. A floating ceiling is hung from the fixed ceiling, and parts of floating walls are hung from overhead beams. Only the floating floor and the other parts of floating walls are set upon the fixed floor. Consequently, the floating floor in the effect sound space was made thicker than would be possible otherwise. 


\title{
Session $3 \mathbf{a A B}$
}

\section{Animal Bioacoustics: Acoustic Scene Analysis and Communication in Animals}

\author{
Cynthia F. Moss, Cochair \\ Univ. of Maryland, Dept. of Psychology, Inst. for Systems Research, College Park, MD 20742 \\ Hiroshi Riquimaroux, Cochair \\ Doshisha Univ., 1-3 Tatara Miyakotani, Kyotanabe, Kyoto 610-0321, Japan \\ Invited Papers
}

3aAB1. Auditory streaming and the spectrotemporal analysis of sound in the cortex. Shihab Shamma and Mounya Elhilali (Elec. and Comput. Eng. \& Inst. for Systems Res., Univ. of Maryland, College Park, MD 20742)

Auditory streaming is a phenomenon that manifests itself in the everyday ability of humans and animals to parse complex acoustic information arising from multiple sound sources into meaningful auditory streams. For instance, the ability to follow a conversation at a noisy cocktail party or hear the violin in the orchestra both rely on the formation of auditory streams. While seemingly effortless, the neural mechanisms underlying auditory streaming remain a mystery. In this talk, we shall discuss how this perceptual ability may emerge as a consequence of the multiscale spectrotemporal analysis of sound in the auditory cortex. A simplified model of this process is developed to demonstrate how auditory streaming could underlie the separation of complex sounds from two sources (e.g., speech or music). The model reveals that certain perceptual attributes are critical for the simultaneous separation of sounds (pitch and onset sensiticity), whereas others are important for the sequential binding of the segregated sound features (timbre and location).

8:05

3aAB2. Time-sharing acoustic stream analysis to recognize multiple objects in space by echolocating bats. Hiroshi Riquimaroux (Doshisha Univ., 1-3 Tatara Miyakotani, Kyotanabe, Kyoto 610-0321, Japan)

Echolocating bats need to catch prey while they have to avoid obstacles and escape from their predators at the same time. Therefore, they have to perceive precise information about multiple objects from their surrounding space while they are flying. However, there are strong restrictions in the system; the neural system is too slow to perform real-time processing, and the bat has only one sound source emission and only two ears to receive echoes. The purpose of the present study was to investigate how echolocating bats perceive important information about different objects in space during their flight by analyzing acoustic characteristics of emitted pulses and returning echoes. Pulses and echoes of CF-FM and FM bats were recorded by a wireless telemetry microphone system (Telemike) on their head. In order to reconstruct their flight trajectory, a dual high speech video camera system was used. Obtained data showed multiple streams for temporal change in echo delay, Doppler-shift compensation, and echo intensity compensation. The present findings indicate that echolocating bats may adopt a time-sharing analysis, which is practically fast enough to recognize multiple objects in space during flight. [Work partly supported by the Innovative Cluster Creation Project promoted by MEXT and by a grant to RCAST at Doshisha University from MEXT.]

3aAB3. Echolocating bats control the directionality of sonar signals to sort out the auditory scene. Annemarie Surlykke (Inst. of Biol., Univ. of Southern Denmark, DK-5230 Odense M., Denmark) and Cynthia F. Moss (Univ. of Maryland, College Park, MD 20742)

A primary function of hearing is the localization and identification of sound sources in the auditory scene. Results from humans and other animals have shown that spectral and temporal features, as well as source direction, are important parameters used for organizing acoustic sources. Echolocating bats control the features of vocalizations to shape information carried by echoes from auditory objects. Our research on big brown bats, Eptesicus fuscus, suggests the importance of vocal control for scene analysis: When a bat performs a dual task, requiring both obstacle avoidance and prey capture, it solves the task sequentially, adapting the temporal pattern of its cries to produce sonar strobe groups, strobing first the obstacle and then the prey. Directional control over the sonar beam pattern confirms this sequential processing: The bat alternates fixation of the beam axis on the obstacle and prey. The importance of directionality is corroborated by studies in another bat, Trachops cirrhosus, where behavior and morphology optimize sonar directionality for different tasks. When hanging from a perch T. cirrhosus emits sounds for general orientation with limited demands for directionality. When hunting, prey localization necessitates high directionality associated with sound emission through the nostrils surrounded by the moveable nose leaf. 
3aAB4. Wild dugongs' vocal responses to conspecific calls. Kotaro Ichikawa, Nobuaki Arai (Biosphere Informatics, Grad. School of Kyoto Univ., Yoshida-honmachi, Sakyo-ku, Kyoto, 606-8501, Japan, ichikawa@bre.soc.i.kyoto-u.ac.jp), Tomonari Akamatsu (Natl. Res. Inst. of Fisheries Eng., Kamisu, Ibaraki, 314-0408, Japan), Tomio Shinke (System Intech Co., Ltd., Shizuoka, Shizuoka, 424-8610, Japan), and Kanjana Adulyanukosol (Phuket Marine Biological Ctr., 83000, Phuket, Thailand)

Wild dugongs were found to call back more to conspecific calls than to artificially synthesized sounds. The population was exposed to four different playback stimulii a wild dugong chirp, a synthesized down-sweep sound similar to the dugong chirp, a synthesized constant-frequency sound, and no sound as a control. Vocalizing dugongs were localized using an array of stereounderwater-recording systems. Wild dugongs vocalized more frequently after the playback of dugong chirps ( 2.8 calls/min) than those of constant-frequency ( 0.55 calls $/ \mathrm{min})$ and control $(0.2$ calls $/ \mathrm{min}),(p>0.01$, Kruskal-Wallis test). Dominant frequencies of response calls were $4810 \mathrm{~Hz}$ to dugong chirps and $4470 \mathrm{~Hz}$ to down-sweep sounds. These were higher than those to other stimuli (3794 and $4044 \mathrm{~Hz})$. Distances of calling-back dugongs from the playback speaker were significantly shorter for dugong chirps $(10.19 \mathrm{~m})$ and down-sweep $(19.02 \mathrm{~m})$ than that for constant frequency $(105.84 \mathrm{~m})(p>0.001)$. The observed dominant frequencies of response calls $(4510 \mathrm{~Hz})$ were above the cutoff frequency of Lloyd's mirror effect in shallow waters of the present study. Frequency-modulated narrow-band sounds like chirps travel longer and enable accurate measurements of source directions by binaural receivers. These suggest that chirps could be used for conspecific recognition in noisy acoustic scenes in tropical shallow waters.

\section{9:05}

3aAB5. How does a green leafhopper male induce a female's willingness to mate? Masao Fukui (Lab. of Insect Physiol.., Grad. School of Agriculture, Kyoto Univ., Sakyo-ku, 606-8502, Japan)

When on their host plants, male leafhopper Nephotettix nigropictus (Insecta; Hemiptera) produces substrate-borne signals of two kinds, which are associated with different behavioral activities: (i) a calling signal and (ii) a duetting signal in response to a female. These signals both influence pair formation and the female's willingness to mate. After receiving a vibrational reply from a female responding to the calling signal, the calling male increases the first component of the calling signal from one trill to two or more trills, then continues in a duet with the female. During more duets, males wanting females to mate increase the number of chirps that compose a buzz as the second component of the calling signal. This increment of the first component is almost always one stable trill, but the increment of the second component apparently depends on the strength of the female's reply. This paper presents discussion of the biological significance and role of male signals to induce females to mate.

\section{Contributed Papers}

9:25

3aAB6. An avian cocktail party: Masking and unmasking in birds. Micheal Dent, Elizabeth McClaine (Dept. of Pyschology, B76 Park Hall, Unversity at Buffalo, Buffalo, NY 14260, mdent@buffalo.edu), Barbara Shinn-Cunningham, Virginia Best, Erol Ozmeral, Frederick Gallun, Kamal Sen, and Rajiv Narayan (Boston Univ., Boston, MA 02215)

The ability to effectively communicate in a noisy environment is an auditory skin that all animals must develop for basic survival. Birds are one group of animals that use auditory communication for everything from mating to prey capture. In general, how well humans and other animals are able to hear signals in their environment is a function of the properties of the signal and the properties of the noise, and include spatial, temporal, and spectral characteristics. Here, the abilities of birds to correctly identify zebra finch songs embedded in different types of maskers that were emitted from the same location or from separate locations (and presented at different signal to noise ratios) were measured using operant conditioning techniques. In general, zebra finches and budgerigars showed very small differences in thresholds between songs embedded in the three types of maskers: broadband noise, modulated noise, and choruses of zebra finch songs. When the calls were emitted from one location and the maskers from another, a condition that elicits spatial unmasking in humans (Best et al., 2005), zebra finches had lower thresholds while budgerigars generally did not. These results suggest some species-specific advantages to listening in noisy environments.
9:40

3aAB7. Assessing the relative importance of source distance determined by acoustic cues and noise level as predictors of disturbance in a study of the Mexican spotted owl. Ann E. Bowles, Stefanie K. Moffat, Samuel L. Denes (Hubbs-SeaWorld Res. Inst., 2595 Ingraham St., San Diego, CA 92109, abowles@hswri.com), Christopher M. Hobbs (Wyle Labs., Arlington, VA 22202), and Dana H. Banwart (GeoMarine, Inc., Hampton, VA 23666)

In forested areas, acoustic localization of source distance and noise level are potentially reliable indicators of an approaching source. The relative importance of these cues was examined during a study of Mexican spotted owl responses to human-made noise. The study was conducted in mixed-conifer forest on steep northeast facing slopes. Owls had no or limited experience with the experimental paradigm. Shaped noise bursts were projected from a Sound Projections portable stereo speaker system with line of sight to the target owl(s). Signals were shaped noise bursts with $10-15-\mathrm{dB} / \mathrm{s}$ onset rate projected to produce received maximum A-weighted levels in two ranges, $55-65 \mathrm{~dB}$ and $75-85 \mathrm{~dB}$, at distances of 20,40 , and $80 \mathrm{~m}$. Levels were measured a short distance from the target owl(s) using a calibrated recording system (DAT or digital recorder equipped with an ACO 7013 microphone, $20 \mathrm{~Hz}$ to $20 \mathrm{kHz}$ ). They were also estimated using a single-event sound propagation model (NMSIM,Wyle Laboratories). Preliminary analysis showed that both source level and distance were significantly related to owl response, but that distance explained twice as much variance in behavior as level. [Work supported by ACC/CEVP; USFWS Permit No. TE024429.] 


\section{9:55-10:10 Break}

\section{0:10}

3aAB8. Comparisons of song perception in male and female songbirds utilizing cardiac responses. Maki Ikebuchi (Human Information System Labs., Kanazawa Inst. of Technol., Hakusan, Ishikawa 924-0893, Japan, mikebuch@his.kanazawa-it.ac.jp) and Kazuo Okanoya (RIKEN Brain Sci. Inst., Wako, Saitama 351-0198, Japan)

In most songbirds, males sing to defend territories and to attract females, and females evaluate the quality of males based on songs. This should lead to differences in song perception between the sexes, but it has been difficult to devise an assay that can evaluate song perception without introducing motivational bias. For example, the copulation solicitation assay is useful for evaluating female perception of songs, but this assay cannot be utilized for males. We found that birds react to a sound of interest by increasing the heart rate, and this response was used to compare song perception in Bengalese and zebra finches. We used conspecific and hetero-specific songs as stimuli and these were presented in a planned order. In Bengalese finches, only females responded to the changes in conspecific song repertoire. In zebra finches, both males and females reacted to such changes. Bengalese finches are a domesticated species and males do not establish breeding territories. Conversely, male zebra finches establish a small territory when breeding. In both species, songs are an important trait by which females select males. These ecological differences concur with the species and sex differences in the cardiac response. [Work supported by JSPS and JST.]

\section{0:25}

3aAB9. Detection of objects in complex environments by echolocating big brown bats. Caroline M. DeLong, Sarah A. Stamper, and James A. Simmons (Dept of Neurosci., Brown Univ., Box 1953, Providence, RI 02912)

In previous psychophysical experiments, big brown bats detected or discriminated objects (monopole and dipole targets comprised of $15 \mathrm{~mm}$ diameter cylinders) presented standing on smooth surfaces with little clutter. Performance was determined by mutual masking of echoes from the targets themselves. In new experiments, bats did two-alternative (left/ right) forced choice tasks to detect a two-cylinder dipole target in complex, cluttered environments. In experiment 1 , the targets were set inside 14-mm-deep, 20-mm-diam holes in a layer of foam $25 \mathrm{~mm}$ thick. Cylinders of different heights protruded 13,5 , or $2 \mathrm{~mm}$ above the surface of the foam, or were recessed $1 \mathrm{~mm}$ below the surface. The bats performance varied as a function of protruding height. In experiment 2, the dipole target was embedded within arrays of distractor objects that varied in shape, size, orientation, and material. Both experiments explore how bats isolate the echoes from the dipole target from the mixture of echoes returning from the clutter-whether distractor objects or echoes from the sides of the holes. Big brown bats must engage in this acoustic scene analysis when catching insects, such as beetles, against a backdrop of foliage.

\section{0:40}

3aAB10. The sonar beam of big brown bats (Eptesicus fuscus) during landing. Jens C. Koblitz, Peter Stilz, Wiebke Pflästerer, Mariana Melcon, and Hans-Ulrich Schnitzler (Tierphysiologie, Universität Tübingen, Auf der Morgenstelle 28, 72076 Tübingen, Germany, jens.koblitz@web.de)

The parameters signal duration, pulse interval, and frequency have previously been used to describe the approach behavior of landing bats. An array with 16 microphones was used to investigate additional parameters during the approach flight of landing big brown bats (Eptesicus fuscus). For each signal emitted the position of the landing bat in relation to the microphone array was determined using a 3-D video system, and the width, maximum intensity, and aiming direction of the sonar beam were calculated. The approach to the landing site begins at a distance of about
$1.4 \mathrm{~m}$ when the bats pick up the landing site with their sonar beam. This focusing on the landing site is indicated by a clear decrease of the deviation between beam aiming and direction to the landing position. The maximum intensity of the sonar beam is reduced by about $6 \mathrm{~dB}$ per half distance during the last $2 \mathrm{~m}$ of the approach, which indicates a gain control mechanism. The width of the sonar beam remains constant throughout the whole approach.

\section{$10: 55$}

3aAB11. Spatial release from masking of aerial tones in a California sea lion (Zalophys californianus). Marla M. Holt and Ronald J. Schusterman (UCSC Long Marine Lab., 100 Shaffer Rd., Santa Cruz, CA 95060)

Spatial release from masking (SRM) occurs when a signal and masker are spatially separated, resulting in improvement of signal detection relative to when they are spatially co-located. Sea lions forage in the water, breed on land, produce airborne vocalizations that are associated with social and reproductive activities, and possess highly reduced pinnae. In this study, SRM was measured at 1,8 , and $16 \mathrm{kHz}$ in a California sea lion who had to detect an aerial tone in the presence of an octave band of white noise centered at the tone frequency. Testing was conducted in a hemianechoic chamber. While the masker always occurred in front of the subject $(0 \mathrm{deg})$, the tone occurred at 0,45 , or $90 \mathrm{deg}$ in the horizontal plane. Absolute thresholds were also measured at these angles to account for differences in hearing sensitivity based on source azimuth. Masked thresholds were lower by as much as $12 \mathrm{~dB}$ at $1 \mathrm{kHz}$ when the signal and masker were separated by $90 \mathrm{deg}$. These results were compared with those of a harbor seal who, like all true seals, naturally lacks pinnae. Performance differences between the two subjects at the highest frequency likely reflect variations in pinna anatomy

\section{$11: 10$}

3aAB12. Underwater noise and zones of masking with respect to hopper dredging and manatees in the St. Johns River in Jacksonville, FL. Edmund R. Gerstein, Joseph E. Blue (Leviathan Legacy Inc., 1318 SW 14th St., Boca Raton, FL 33486), Gerard F. Pinto, and Seth Barr (Jacksonville Univerisity, Jacksonville, FL 32211)

Underwater noise radiating from dredging can effectively obscure or mask biological and other important sounds. This study recorded underwater acoustic characteristics of hopper dredging in the St. Johns River, Jacksonville, FL, to evaluate noise impacts in the waterway with respect to the endangered West Indian manatee. Of particular interest was the extent and range that dredging noise may mask the sounds of approaching commercial and recreational vessels. Vertical hydrophone arrays and a multichannel PC-based recording system were used to measure dredging noise at various distances. Ambient noise surveys, active propagation of calibrated sources, and controlled boat noise measurements were conducted along the waterway. These data were integrated with behavioral hearing data to estimate zones of masking surrounding dredging. Three discernable noise sources that masked boat noise were (1) cavitation from dredge propellers, (2) draghead vacuuming, and (3) noise from submerged slurry pipelines. Sustained high ambient noise levels from dredging can significantly increase the risk of manatee-boat collisions by masking the sounds of approaching vessels over large radii (up to 2.5 miles). Mitigations suggested include ship quieting, reducing propeller cavitation, insulating or elevating slurry pipelines, and minimizing transects to pump out stations. [Work funded by the City of Jacksonville Waterways Commission.] 
3aAB13. "Vocal grooming" in an eusocial subterranean rodent: The naked mole-rat (Heterocephalus glaber). Shigeto Yosida, Kazuo Okanoya (Grad. School of Sci. \& Technol., Chiba U., 1-33 Yayoi-Cho, Inage, Chiba, 263-8522 Japan), Chizuko Suzuki, Sanae Nanvuh, and Kazuo Okanoya (RIKEN BSI., Wako, Saitama, 351-0198 Japan)

The naked mole-rat is a unique communal-living rodent of east Africa. In a subterranean environment, this small rodent species has a complex hierarchical society with labor division that is comparable to those of bees and ants. They are also known for their extensive vocal repertory of more than 17 sounds, which might have evolved as an alternative to visual cues, and which contributes to maintaining its society. We showed that their most frequent vocalization, the soft-chirp (SC), has an antiphonal nature, and it might function for rank-identification and reconciliation purposes. The SC is elicited either by another's SC or physical touch. We analyzed acoustic properties of SC elicited by touching the animals, and found that it varies according to the body mass. The emission rate was highest among larger (superior) animals, but the smaller (inferior) of the two emitted SCs more frequently when observing behavior of two interacting animals. Playback experiments revealed that the use of SC generally follows these two rules: smaller animals vocalize more often than larger ones, and larger ones respond only to smaller animals' SCs. These features of SC resemble grooming behavior among primates.
3aAB14. Neural responses to communication sound in the inferior colliculus of the naked mole-rat. Shizuka Tokin, Kohichi Tanaka (Lab. for Molecular Neurosci., Sch. of Biomed. Sci., Tokyo Med. and Dent. Univ., 1-5-45 Yushima, Bunkyo-ku, Tokyo 113-0034 Japan), Shizuka Tokin, and Kazuo Okanoya (RIKEN BSI., Wako, Saitama, 351-0198 Japan)

The naked mole-rat (Heterocephalus glaber) is a highly social subterranean rodent that lives in Eastern Africa. Because they a dig burrow system and spend most of their lives underground, they are functionally blind and rely heavily on auditory information. They have at least 17 different vocalizations, which are associated with specific behavioral contexts. In this study, we focused on the soft chirp (SC), which is one of the most frequent vocalizations in this species, and recorded neural responses to synthesized SCs in the inferior colliculus. We found that the responses could be divided into three groups based on their response patterns. These response patterns represented the duration or frequency of stimuli. In addition, we also found that the number of spikes changed with the degree of frequency modulation. From these results, we hypothesized that the vocalizations of naked mole-rats can be represented by integrating the activity of such neurons that respond to a specific feature of sound.

Session 3aBB

\title{
Biomedical Ultrasound/Bioresponse to Vibration and Physical Acoustics: Celebration of Floyd Dunn I
}

\author{
William D. O'Brien, Cochair \\ Univ. of Illinois, Dept. Electrical and Computer Engineering, 405 N. Mathews, Urbana, IL 61801 \\ Junichi Kushibiki, Cochair \\ Tohoku Univ., Dept. of Electrical Engineering, 6-6-05 Aoba, Aramaki-Aza, Aoba-ku, Sendai 980-8579, Japan
}

Chair's Introduction-8:00

Invited Papers

8:05

3aBB1. Celebration of Floyd Dunn: Some remarks about his life, career, and accomplishments. William D. O'Brien, Jr. (Bioacoustics Res. Lab., Dept of Elec. and Comput. Eng., Univ. of Illinois, 405 N. Mathews Ave., Urbana, IL 61801)

No celebration of Floyd Dunn would be complete without some remarks about the life, career, and accomplishments of this remarkable scientist/engineer. Floyd had made significant scholarly contributions in six general themes, and you will hear about their impact to our welfare during the celebration. The six ultrasonic biophysics themes include absorptive processes, nonlinear phenomena, application in living systems, toxicity, measurement techniques, and ultrasonic microscopy. Floyd has been recognized for his accomplishments by being the recipient of most of the important national and international awards including National Academy of Sciences, National Academy of Engineering, ASA's Gold Medal and Silver Medal in Bioresponse to Vibration, Acoustical Society of Japan's Medal of Special Merit, IEEE's Edison Medal, and election to Fellowship in six professional societies.

\section{$8: 25$}

3aBB2. Imaging propagation speed of pulsive vibration in the heart wall for healthy subjects and patients with old myocardial infarction. Hiroshi Kanai (Dept. of Electron. Eng., Tohoku Univ., Aramaki-aza-Aoba 6-6-05, Sendai 980-8579, Japan), Sachiko Watanabe (Miyagi Shakaihoken Hospital, Sendai, Japan), Yoshifumi Saijo, and Motonao Tanaka (Tohoku Univ., Sendai, Japan)

This study presents a novel method that noninvasively visualizes myocardial heterogeneity for healthy subjects and patients with old myocardial infarction. By a sparse sector scan, the myocardial minute motions were measured simultaneously at about 1000 points in the heart wall at a high frame rate. We have found that a pulsive vibration is excited by aortic-valve closure [H. Kanai and $\mathrm{Y}$. Koiwa, Ultrasound Med. Biol. 27, 481-498 (2001)]. The consecutive spatial distributions of the vibrations reveal that the vibration propagates from the root of the aortic valve to the apex [H. Kanai, IEEE Trans. UFFC. 51, 1931-1942 (2005)]. In this study, the 
method was applied to seven healthy subjects and five patients, and the spatial distribution of the propagation speed of the vibration is also visualized. For all healthy subjects, the propagation of the pulsive vibration was visible both in longitudinal-axis and apical views, and there is spatial difference in the propagation speed; $4.3 \pm 0.5 \mathrm{~m} / \mathrm{s}$ for the high echo area and $2.9 \pm 0.4 \mathrm{~m} / \mathrm{s}$ for the low echo area, for a $60-\mathrm{Hz}$ component. For patients, the propagation speed in the diseased area is about $30 \%$ lower than the normal area. These preliminary results indicate that the method has a potential for revealing myocardial heterogeneity.

\section{8:45}

3aBB3. Ultrasound stimulated vibroacoustography and vibrometry for measuring tissue properties. James F. Greenleaf and Chen Shigao (Mayo Clinic College of Medicine, Rochester, MN 55901)

Ultrasound stimulated vibroacoustography has been used to image tissues using the acoustic response of tissues to localized harmonic radiation pressure. The method provides high resolution and high dynamic range images of tissues. The parameter being imaged is a complex combination of scattering, attenuation, and nonlinearity. However, specific use of harmonic or pulsed radiation pressure and subsequent measurement of the tissue response can be used to measure fundamental material properties of tissue. The measurement of shear wave dispersion can be used to estimate elastic shear moduli of tissue. Fundamental model free properties such as elastic storage and loss constants can also be measured. Ultrasound radiation pressure is used to induce free propagating shear waves. The measurable properties of the shear waves are sensitive to only the material properties of the tissue under certain circumstances. Shear moduli in tissue are measured with high accuracy and precision given appropriate models of wave propagation within the geometry of the tissue. Careful use of shear wave propagation and subsequent measurements can provide fundamental measurements of tissue mechanical properties if models are accurate.

9:05

3aBB4. Evaluation of liver fibrosis using the acoustic characteristic and statistical signal processing of echo. Tadashi Yamaguchi (Faculty of Eng., Chiba Univ., 1-33 Yayoi-cho, Inage-ku, Chiba, 263-8522 Japan) and Hiroyuki Hachiya (Chiba Univ., Inage-ku, Chiba, 263-8522 Japan)

We evaluate the relationship between changes in acoustic properties accompanying change of a tissue and echo information using human liver specimens in vitro. The rf echo signals of a 20 -mm-thick human liver specimen (200 frames) were acquired continuously using a probe using $2.0-\mathrm{MHz}$ transmission frequency and $4.0-\mathrm{MHz}$ receiving frequency. The signals were processed using statistical signal processing to extract abnormal echo information. After ultrasonic scanning, $50 \times 50-\mathrm{mm}^{2}$ sections were cut from the liver specimens for microscopy and acoustic microscope measurement. The liver fiber structure was extracted from $204-\mu \mathrm{m}$-thick Masson's trichrome stained microsections. The speed of sound and the attenuation of 2-mm-thick cut specimens were measured using 25-MHz ultrasound. Results of comparing 3-D structures of abnormal information extracted from echo signals and the 3D fiber structures extracted from microsections showed that both structures were clearly in agreement. Furthermore, it was confirmed that the speed of sound of fibers measured using an acoustic microscope was faster than that for normal liver tissue.

9:25

3aBB5. The importance of cells and arrangement of cells to ultrasound backscatter. Michael Oelze (Dept. of Elect. \& Comput. Eng., Univ. of Illinois at Urbana-Champaign, 405 N. Mathews, Urbana, IL 61801, oelze@uiuc.edu)

The early work of Professor Floyd Dunn identified the importance of collagen structures and globular protein to ultrasound backscatter [Fields and Dunn, J. Acoust. Soc. Am. 54, 809-812 (1973)]. Recent advances in tissue characterization through ultrasound backscatter have again focused on determination of the underlying microstructures responsible for scattering. The focus of new models for ultrasound backscattering has been on the contribution of cells. Specifically, new models have sought to address the ultrasound backscatter from single cells and from different arrangement of cells. The importance of cells and their arrangements in tissues to classification of disease, i.e., cancer, through ultrasound was also noted by Professor Dunn [Saijo et al., Ultrasound Med. Biol. 17, 709-714 (1991)]. In the present work, the construction of a new cell model of scattering was made from measurements of acoustic properties of cells. Different arrangements of cells, i.e., uniform distribution of cells and cells arranged in groups, were examined in simulation using the new cell model. Finally, the new cell model and simulations were compared to ultrasound backscatter measurements from different kinds of cancer. The arrangement of cells within a cancerous region was observed to be significant for diagnostically differentiating between cancers using ultrasound backscatter.

\section{9:45}

3aBB6. Evaluation of blood flow velocity vector measurement accuracy: Numerical simulation and flow phantom studies of Rankine's vortex. Takashi Okada, Teruyuki Sonoyama, Akimitsu Harada (Aloka Co., Ltd., 3-7-19, Imai, Ohme, Tokyo, 198-8577, Japan), Shigeo Ohtsuki (Tokyo Inst. of Technol., Nagat-suda, Midori-ku, Yokohama, 226-8503, Japan), and Motonao Tanaka (Tohoku Employee's Pension Welfare Hospital, Hukumuro, Miyagino-ku, Sendai, 983-8512, Japan)

A new method to measure the velocity vector of blood flow on the observation plane was recently developed [Ohtsuki and Tanaka, J. Visuali. 9, 69-82 (2006)]. The method is based on the calculation of stream functions. To evaluate the accuracy of this method, we have studied the velocity distribution of Rankine's vortex in numerical simulation and experiments. The vortex of the simulation model was $50 \mathrm{~mm}$ in radius, the forced vortex was $20 \mathrm{~mm}$ in radius, and the maximum velocity of the vortex was $50 \mathrm{~cm} / \mathrm{s}$. The differences between the velocity profiles of the vortex obtained by this method and by simulation were less than 10\%, although the accuracy depended on the angle of the observation plane. Furthermore, we conducted phantom experiments in which the vortex was created in degassed water by using a magnetic stirrer. Color Doppler data of the vortex flow were acquired using a ultrasonic diagnostic system (SSD-6500, Aloka, Japan) and the velocity vector distribution was calculated. The experimental results were in good agreement with the simulation model. We conclude that the reliability of this method is very high. The results suggest great potential of the new velocity vector method for quantitative diagnosis of cardiac functions. 
3aBB7. Professor Floyd Dunn's contribution to understanding the biological effects of ultrasound. Mel E. Stratmeyer (Food and Drug Administration/CDRH, 9200 Corporate Blvd. (HFZ-120), Rockville, MD 20850, melvin.stratmeyer@fda.hhs.gov)

During Professor Dunn's career, ultrasound exposure has evolved from being used to induce dramatic tissue effects in animals to routine diagnostic fetal imaging in humans. An understanding of the fundamental mechanisms and dosimetric parameters involved in ultrasound-induced biological effects was essential to allow ultrasound to be clinically used for tissue destruction, physical therapy, and routine diagnostic imaging. Professor Dunn's early research and the research of other prominent scientists, many of them trained by Professor Dunn, will be discussed to demonstrate advancements in our understanding of the biological effects of ultrasound.

\section{0:40}

3aBB8. Reduction of cavitation threshold by second-harmonic superimposition and its potential application to sonodynamic therapy. Shin-ichiro Umemura (School of Health Sci., Kyoto Univ., Kyoto, 607-8507 Japan, sumemura@hs.med.kyoto-u.ac.jp), Ken-ichi Kawabata, Kazuaki Sasaki, and Nami Sugita (Hitachi Central Res. Lab., Tokyo, 185-0043 Japan)

Acoustic cavitation is known to induce bioeffects under certain conditions. Cavitation threshold, the acoustic strength needed to induce such cavitation, in various biological systems was intensively studied by Dr. Dunn's group. Their results have significantly contributed to the safety concerns of diagnostic and therapeutic uses of ultrasound. Acoustic cavitation is also known to be the primary cause of sonochemical reactions. Chemical compounds such as hematoporphyrin were found to be activated by acoustic cavitation and induce in vitro and in vivo antitumor effects thereby. In order to make use of this effect in therapeutic practice, cavitation must be generated reproducibly without depending on standing waves at acoustic power inducing no significant heating. It was also found that the cavitation threshold can be significantly reduced by superimposing the second harmonic onto the fundamental in insonation. The threshold was reduced by more than an order of magnitude, especially in the presence of a certain compound such as Rose Bengal (RB). An amphiphilic derivatives of RB, selectively accumulating in tumor tissues, was synthesized and used in combination with focused ultrasound with second-harmonic superimposition to treat experimental murine tumors, resulting in significant reduction of their sizes. A hypothesis on the mechanism behind this will also be discussed.

\section{1:00}

3aBB9. Exposure estimation and dosimetry for ultrasound thermal therapies. Gail ter Haar (Joint Dept. of Phys., Inst. of Cancer Res., Royal Marsden Hospital, Sutton, Surrey, SM2 5PT, UK)

The success of ultrasound thermal therapies relies on achieving a predetermined temperature rise in a specified tissue volume. If these treatments are to be used in clinical application, it is important to be able to estimate the required exposure parameters and to undertake appropriate dosimetry. Floyd Dunn and his co-workers were pioneers in this field for high-intensity focused ultrasound (HIFU). In early papers they addressed the problem of ultrasonic dosage and dose rate. This remains a topic of active discussion. A major part of these investigations relies on accurate temperature measurement in ultrasonic fields in tissue. The viscous heating artifact that occurs when thermocouples are used was first recognized by the Illinois group in the 1950s. Effective ways of estimating this component of the measured temperature rise are still being sought. Investigation of the dependence of HIFU lesion size on in situ ultrasound intensity, exposure time, and frequency will be described, and the importance of acoustic cavitation and boiling for the definition of an ultrasound dose parameter will be discussed.

\section{1:20}

3aBB10. Numerical analysis of temperature in tissue radiated by focused ultrasound. Nobuyuki Endoh and Takenobu Tsuchiya (Dept. of Eng., Kanagawa Univ., Yokohama 221-8686, Japan)

Higher power ultrasonic equipment is recently extending not only into therapy but also into diagnosis because newer diagnostic systems such as harmonic imaging require higher ultrasound than conventional imaging systems. It is very important to assure the safety in the use of diagnostic equipment, especially for prevention of burns caused by radiation from ultrasonic imaging systems. In this two-dimensional FDTD-HCE study, temperature increases in tissue were simulated at the focal point of a phased array transducer like a common B-mode imagine system. A center frequency of radiated pulse is assumed to be $2.5 \mathrm{MHz}$ and ISTPA $=0.72 \mathrm{~W} / \mathrm{cm}^{2}$. Results show that the temperature in tissue at the focal point is proportional to the pulse repetition frequency (PRF) when the PRF is changed from 100 to $400 \mathrm{kHz}$. It is also shown that common equipment is safe because the maximum temperature rise in tissue is only $0.0004^{\circ} \mathrm{C}$ at the focal point of the transducer even though the PRF is $400 \mathrm{kHz}$. [Work supported by High-Tech Research Center Project from the Ministry of ECSST.]

\section{1:40}

3aBB11. Professor Floyd Dunn's contributions to ultrasound metrology. Gerald R. Harris (U.S. Food and Drug Administration, 12725 Twinbrook Pkwy., Mail Code HFZ-170, Rockville, MD 20852-1719, gerald.harris@fda.hhs.gov)

A half-century ago the applications of ultrasound in medicine were, apart from physiotherapy, mostly experimental, and the related bioeffects and toxicity studies were in their infancy. Progress in both of these areas was hampered in part by the lack of acceptable tools for measuring the exposure levels, especially for the intense fields then being used in ultrasound surgical studies. Professor Floyd Dunn's advancements in ultrasound measurement techniques at the University of Illinois Bioacoustics Research Laboratory afforded accurate means for quantifying these exposures and laid the groundwork for subsequent developments in ultrasound measurement and calibration. In this presentation Professor Dunn's early work in thermal and radiation force techniques will be described and some recent methods that are being investigated to characterize ultrasound fields, particularly at high intensities, will be reviewed. 


\title{
Session 3aEA
}

\section{Engineering Acoustics, ASA Committee on Standards, and Psychological and Physiological Acoustics: Hearing Aids}

\author{
Stephen C. Thompson, Cochair \\ Pennsylvania State Univ., Applied Research Lab., P.O. Box 30, State College, PA 16804 \\ Yasuhiko Arai, Cochair \\ Kanazawa Inst. of Technology, 7-1 Ohgigaoka, Nonoichi, Ishikawa 921-8501, Japan
}

Invited Papers

\begin{abstract}
8:00
3aEA1. Digital hearing aids: A brief history. Harry Levitt (P.O. Box 610, Bodega Bay, CA 94923)

The journey leading to the development of digital hearing aids began at Bell Laboratories in the mid 1960s when a digital computer was used to simulate an experimental high gain telephone with frequency shaping for people with hearing loss. The simulation was off-line at about 100 times real time. About 20 years later, a digital master hearing aid operating in real time was developed using a high speed array processor. The development of wearable digital hearing aids followed soon after as high speed digital signal processing (DSP) chips were developed. The first wearable digital hearing aid was a body worn instrument and was not a commercial success. At this stage, digitally controlled analog hearing aids could be made small enough to be worn on the ear and these instruments were widely used by the end of the decade. During the 1990s, low-power DSP chips were developed that were small enough to allow for all-digital ear-worn hearing aids to be developed. The challenge today is to tap the tremendous potential of digital signal processing techniques within the power and size constraints of a practical, wearable instrument.
\end{abstract}

3aEA2. A new speech enhancement method for two-input two-output hearing aids. Junfeng Li, Shuichi Sakamoto, Yo-iti Suzuki (R. I. E. C., Tohoku Univ., 2-1-1, Katahira, Sendai, 980-8577, Japan, junfeng@ais.riec.tohoku.ac.jp), and Satoshi Hongo (Miyagi Natl. College of Tech., Medeshima-Shiote, Natori-shi, Miyagi, 981-1239, Japan)

Human beings have the ability to pick up a speech signal in noisy environments, which is known as the cocktail-party effect. This phenomenon is, however, often degraded in persons with impaired hearing. Therefore, a good method to enhance this phenomenon must be very useful to improve speech intelligibility in ambient noise for hearing-impaired persons. In this lecture, multi-input two-output speech enhancement techniques are first summarized. Subsequently, a new two-input two-output speech enhancement method is proposed that is based on the frequency domain binaural model (FDBM) [Nakashima et al., Acoust. Sci. Technol. 24, 172-178 (2003)]. In the proposed method, the interaural differences are first calculated from the noisy observations and employed to determine the sound-source locations. A speech absence probability (SAP) estimator is then developed using knowledge of estimated source locations. It is further integrated into the original FDBM to improve the interference-suppression capability. Effectiveness and superiority of the proposed speech enhancement method with respect to the original FDBM are validated through comprehensive experiments in terms of objective and subjective speech-quality measures. [Work supported by Sendai Intelligent Knowledge Cluster.]

8:40

3aEA3. Utilizing the auditory systems processing capabilities in the development of methods to auto-steer signal processing strategies in hearing instruments. Andrew B. Dittberner, Jeff Bondy, Maureen Coughlin, Bill Whitmer (GN Auditory Res. Lab., 2601 Patriot Blvd., Glenview IL 60026), and Melissa Dominguez (DBK Acoust., Grayslake IL, 60030)

Hearing instruments have evolved in both the hardware and signal processing domains, providing more computational capabilities and sophisticated sound processing strategies. Static or adaptive methods are employed by these sound processing strategies to achieve a desired output. Due to the adaptive nature of some of these processing strategies and the continual increase in the number of parameters that can interact negatively with each other, there is a need to provide some method of global control over the parameter states. In addition, due to individual needs of the end user, parameter states in a hearing instrument may not satisfy the broad range of acoustic environments that a listener encounters, so identification of particular acoustic environment characteristics is also important. The purpose of this work is to introduce and discuss these problems and some of the current research efforts addressing environmental classification and global control of a hearing instrument's parameter states. The scope of this discussion will be on strategies employed to meet the needs of the end user in reference to the acoustic environment they identify as having a negative impact on their auditory experience and the desired performance they expect from the hearing instrument in that environment. 
3aEA4. A study of signal-to-noise ratio conditions for measuring the intelligibility of hearing aids in noisy live environments. Yasuhiko Arai (Kanazawa Inst. of Technol., 7-1 Ohgigaoka, Nonoichi, Ishikawa 921-8501, Japan, araiyasu@neptune.kanazawa-it.ac.jp) and Yoshiyuki Yoshizumi (Matsushita Electric Ind. Co., Ltd., Tsuzuki-ku, Yokohama 224-8539, Japan)

The authors studied a method to measure the intelligibility of directional hearing aids using a dummy head in noisy environments. The method is based on ISO recommendations (ISO 8353-2, ISO 8353-3). However, it is sometimes difficult to meet the quasi-freefield conditions. Therefore, a method for approximate measurement of intelligibility, by which the equivalent results are obtained in noisy live environments, is recommended. To simulate noisy environments, a multitalker (MULT) noise or a simulated multitalker noise was simultaneously and noncorrelatively put out through four loudspeakers in the live testing room. The MULT noise was composed of eight voices (four males and four females). The experiment showed that mixed voices of eight to ten persons were adequate for interference of the test speech words. The 50\% SNR specification, meaning signal-to-noise ratio that gives intelligibility of $50 \%$ correct, was introduced into the measurement system. Intelligibility was measured under various SNR conditions. It was expressed approximately by the sigmoid function of SNR. The 50\% SNR was estimated from the experimental equation. The intelligibility was measured adequately in the range between $0 \%$ and $100 \%$ by using the $50 \%$ SNR specification. The system configuration, measuring conditions, and measured samples are discussed.

9:20

3aEA5. Perception-model-based sound quality prediction for hearing aids: Big effect, small effect, and the optimization of noise reduction algorithms. Birger Kollmeier, Rainer Huber, Thomas Rohdenburg, and Volker Hohmann (Medizinische Physik, Universitaet Oldenburg and Kompetenzzentrum HoerTech, D-26111 Oldenburg, Germany)

Objective sound quality models can be used to optimize hearing aid algorithms and their respective parameter settings by numerically predicting the subjective sound quality assessed by humans without having to perform quality measurements with humans concurrently. The big effect prediction considers how the (individual) hearing loss affects the aided or unaided perceived sound quality. The small effect prediction aims at subtle differences at a given amplification level of the hearing aid in order to compare processing parameters. This talk will concentrate on benchmarking different types of objective prediction methods both for big effect and small effect conditions using a wide range of signals and processing conditions, respectively. In addition, the performance of PEMO_Q, an auditory-model-based quality prediction scheme developed at Oldenburg University will be discussed, which has been modified to include the effect of hearing impairment and the adaptation to different kinds of test data sets. We will also demonstrate the degree to which PEMO_Q is beneficial for optimizing noise reduction algorithms that are suitable for hearing aids. The objective measure helps to efficiently scan the multidimensional parameter space for an optimum parameter set of the noise reduction algorithm. [Work supported by BMBF and by HoerTech.]

9:40

3aEA6. The design and evaluation of a noise suppression system for custom-made digital hearing aids. Yoshiyuki Yoshizumi and Shigekiyo Fujii (Matsushita Electric Industrial Co., Ltd., 600, Saedo-cho, Tsuzuki-ku, Yokohama, 224-8539 Japan, yoshizumi.yoshiyuki@jp.panasonic.com)

A noise suppression system, which has the inbuilt feature to adapt to the local environment, for custom-made digital hearing aids has been developed. The signal process is based on spectral subtraction technology with 32 bands in the frequency domain. The signal-to-noise ratio was improved by an average of $7.5 \mathrm{~dB}$ in rms level. Its effectiveness was evaluated using the seven-point rating scale method for nine normal-hearing subjects and six hearing impaired subjects. Test speech is presented under three different environments, which consist of a car, a duct, and a crowded place. As a result of the assessment, the same tendency is observed between normal and hearing-impaired subjects and there were two good points that were found. First, the noise suppression system makes them more comfortable to listen to speech with the statistical significance of $p=0.05$. Second, the noisiness is reduced with the statistical significance of $p=0.01$. It should be noted that there is a scattering of the plotted data; however, statistically, the data result is quite acceptable.

10:00-10:20 Break

10:20

3aEA7. Development of novel biologically inspired directional microphones. Ronald N. Miles, Weili Cui, Quang Su, Rui Wu, Lin Tan, Yang Liu, Stephen A. Jones, Venkatesh Mohnankrishnaswami, Thomas Strait, William Butler, David DiBernardo (Dept. of Mech. Eng., State Univ. of New York, Binghamton, NY 13902-6000, miles@ binghamton.edu), F. Levent Degertekin, Baris Bicen, Wook Lee, and Kamran Jeelani (Georgia Inst. of Technol., Atlanta, GA 30332-0405)

The development of novel directional microphones for hearing aids is described. The mechanisms underlying the design of these unusual microphones were inspired by our earlier study of the ears of the parasitoid fly Ormia ochracea [Miles, et al., J. Acoust. Soc. Am. 98, 3059-3070 (1995)]. The structure of Ormia's ears inspired new approaches to the design of directional microphones that have the potential to be more sensitive and have lower thermal noise than typical miniature microphones. The mechanisms for directional hearing in this animal are discussed along with the engineering design concepts that they have inspired. Microphones have been fabricated out of silicon that employ either capacitive sensing or optical sensing to convert the diaphragm motion into an electronic signal. Measured results indicate that the directivity of these microphones is very similar to that of an ideal first-order differential microphone. In addition, novel microphone diaphragms have been fabricated that posses a second-order directional response. These can be used to achieve a significant reduction of unwanted background acoustic noise in hearing aid applications. [Work supported by NIH Grant 5R01DC005762-03, Sensing and Processing for Directional Hearing Aids, to RNM.] 


\section{Contributed Papers}

11:00

3aEA8. Binaural speech enhancement by complex wavelet transform based on interaural level and argument differences. Satoshi Hongo (Dept. of Design and Comput. Applications, Miyagi Natl. College of Technol., 48 Nodayama, Medeshima-Shiote, Natori, Japan, hongo@miyagi-ct.ac.jp) and Yôiti Suzuki (Res. Inst. of Elec. Commun., Tohoku Univ., Japan)

Binaural information might enhance speech signals in noisy environments. Most precedent studies in this area have implemented signal processing in time and frequency domains. For this study, an enhancement method using complex wavelet transform (CWT) was proposed. The CWT has a scale domain whose bandwidth is inversely proportional to the scale level and may therefore be well compared to auditory filters. The proposed processing procedure is the following: (1) By computing CWT for a sound signal at every direction of arrival (DOA), a database (DB) of the scale domain wavelet coefficients is prepared for every DOA. (2) For binaural input signals from an unknown direction, the scale domain wavelet coefficients are calculated using CWT. (3) The DOA of the input signal is estimated as the direction for which interaural level and argument differences calculated from wavelet coefficients are the most similar to those in the DB. (4) The input signal is segregated similarly to FDBM (Nakashima et al., 2003) based on the estimated DOA. Experiments with a directional target signal and an interference sound source calculated from real HRTFs were conducted to show the effectiveness of the proposed algorithm. [This research was partially supported by Sendai Intelligent Knowledge Cluster.]

\section{1:15}

3aEA9. Study of the multimicrophone composed of unidirectional microphones for glasses-type hearing aids. Kenzo Miura (Panasonic MCE Co., Ltd., 4-3-1, Tsunashima-higashi, Kohoku-ku, Yokohama 223-8639, Japan) and Yasuhiko Arai (Kanazawa Inst. of Technol., Nonoichi, Ishikawa 921-8501, Japan)

That multiunit microphones (MUMs) using unidirectional units offer high sensitivity, low electronic noise, and low wind noise for the binaural hearing aids of glasses type is a fact that has already been reported. [K. Miura and Y. Arai, Th.2.D.4, 18th ICA, Kyoto, Japan (April, 2004)]. Two types of MUM are proposed herein for glasses-type hearing aids that have an array of three units positioned over the front frame for each channel. One type is the parallel front-direction array type (PA-MUM). The other type has a twisted direction array (TA-MUM). Performances of these MUMs were compared with that of a bigradient (BG) microphone positioned on the side frame. Results show that the PA-MUM has very narrow directivity; TA-MUM showed moderate directivity. The electronic noise and the wind noise of these MUMs were at a very low level. On the other hand, the BG microphone showed rather sharp directivity, but the electronic noise from the signal conditioner and the wind noise were both of a high level. These results show that PA-MUM is suitable for listening to speech in very noisy environments and that TA-MUM is suitable for general use for glasses-type hearing aids.
11:30

3aEA10. A model of own-voice perception with hearing aid usage. Stefan Stenfelt (Dept of Neurosci. and Locomotion/Div. of Tech. Audiol., Linkping Univ., SE-58185 Linkping, Sweden) and Sabine Reinfeldt (Chalmers Univ. of Technol., SE-41296 Göteborg, Sweden)

Until recently, own-voice issues for hearing aid users were dominated by the occlusion effect. However, with the so-called open fittings, the occlusion-related own-voice problems have been significantly reduced. There are groups of hearing-aid users, though, for whom open fittings are unavailable, either because of high-frequency gain requirements or because of ear geometry. Of even bigger importance is the fact that hearingaid users still have own voice issues, even when their occlusion problems have essentially been solved. A model is presented for perception of the own voice comprising an estimation of the own voice transmission by airborne and structure borne sound. Parameters of the model are the occlusion effect from the ear mould and ventilation tubes, gain settings of the hearing aid, and transmission properties of the middle ear during vocalization. The results show that both the airborne and structure borne sound transmission depend on both amplifier and acoustical parameters. A ventilation tube in the ear-mould may introduce a resonance that causes extreme alterations of the own voice spectral content. Although the model is far from complete, it can provide useful insights on own-voice related issues and give design directions to alleviate, at least partly, own-voice problems related to hearing aid usage.

\section{$11: 45$}

3aEA11. Transverse variations of sound pressure in an ear canal occluded by a hearing aid. Michael R. Stinson and Gilles A. Daigle (Inst. for Microstructural Sci., Natl. Res. Council, Ottawa, ON, Canada K1A 0R6)

The sound field in a model ear canal has been investigated experimentally and theoretically. The canal was cylindrical, 7.5-mm diameter, and terminated with a Zwislocki coupler to represent absorption by the human middle ear. The outer end of the canal was driven by a test hearing aid, with the acoustic output from a receiver entering the canal through a 1-mm port. The hearing aid was provided with a 20-mm-long vent, either 1 or 2 $\mathrm{mm}$ in diameter. The sound field inside the canal was measured using a specially designed 0.2-mm-diam probe microphone [Daigle and Stinson, J. Acoust. Soc. Am. 116, 2618 (2004)]. In parallel, calculations of the interior sound field were performed using a boundary element technique. Both calculations and experiment reveal large transverse variations of sound pressure level, as much as $25 \mathrm{~dB}$ at $8 \mathrm{kHz}$, near the inner face of the hearing aid. Variations are greatest near the outlet port of the receiver and the vent hole. Deeper into the canal, the transverse variations are less significant and, at depths greater than $5 \mathrm{~mm}$, only a longitudinal variation remains. [Work supported by Gennum Corp.] 


\title{
Session 3aNS
}

\section{Noise, Speech Communication, and ASA Committee on Standards: Progress in Hearing Protection and Hearing Conservation I}

\author{
Ellliott H. Berger, Cochair \\ E-A-R Aearo Co., 7911 Zionsville Rd., Indianapolis, IN 46268-1657 \\ Hiroaki Takinami, Cochair \\ RION Co., Ltd., 3-20-41 Higashimotomachi, Kokubunji, Tokyo 185-8533, Japan \\ Chair's Introduction-7:30 \\ Invited Papers \\ $7: 35$
}

3aNS1. Results from the National Institute for Occupational Safety and Health Administration/Environmental Protection Agency (NIOSH/EPA) interlaboratory comparison of American National Standards Institute (ANSI) S12.6-1997 Methods A and B. William J. Murphy (Hearing Loss Prevention Team, NIOSH, MS C-27, 4676 Columbia Pkwy., Cincinnati, OH 45226-1998, wjm4@cdc.gov), Elliott Berger (Aearo/E-A-R Corp., Indianapolis, IN 46268-1657), Dan Gauger (Bose Corp. Framingham MA 01701-9168), Brad Witt (Howard Leight Industries, San Diego, CA 92154), Rich McKinley (U.S. Air Force Res. Labs., Dayton, OH 45433), Samir Gerges (Laboratório de Vibraçöes e Acústica, Florianópolis, SC, Brazil), and William Ahroon (U.S. Army Aeromedical Res. Lab., Fort Rucker, AL 36362-0577)

The Environmental Protection Agency and the National Institute for Occupational Safety and Health sponsored the completion of an interlaboratory study (E-A-RCal ${ }^{\mathrm{SM}}$ Laboratory, Howard Leight Industries, Brazil Acoustics and Vibration Laboratory, NIOSH Robert Taft Laboratories, US Air Force Research Laboratory, and US Army Aeromedical Research Laboratory) to compare two fitting protocols described in ANSI S12.6-1997 (R2002). Method A (informed user fit) and Method B (naive subject fit) were implemented according to the standard. Six models of hearing protection were tested in the study: two earmuffs, foam, premolded, and custommolded earplugs, and canal caps. The muffs were counterbalanced both in testing order and the occluded and unoccluded conditions. The earplugs were counterbalanced across testing order and occlusion. Each laboratory tested 24 subjects for both methods and each protector. This paper reports on the results from this study. Particularly, the comparison of the statistical errors for each method and protector will be evaluated. In general the lower, 80th percentile, estimates of the expected protection were quite similar. The upper, 20th percentile, tended to exhibit effects that were dependent upon the method and the laboratory. [Work supported by EPA interagency agreement DW75921973-01-0.]

\section{$7: 55$}

3aNS2. Methods of measuring the insertion loss of active noise reduction devices. Dan Gauger (Bose Corp., M.S. 271E, 145 Pennsylvania Ave., Framingham, MA 01701, dan_gauger@bose.com), Elliott Berger, Ronald Kieper (E-A-R/Aearo Technologies, Indianapolis IN 46268), John Casali, and Chuck Perala (Virginia Tech, Blacksburg VA 24061)

As active noise reduction (ANR) becomes more common, the need has developed for the measurement of device attenuation, passive and active combined, for use in noise protection calculations. An experiment was conducted involving both REAT and MIRE measurements on five circumaural and two supra-aural ANR devices in two different laboratories. Subject selection and instruction were consistent with the ANSI S12.6-1997 Method B fitting paradigm for all measurements. MIRE was measured both using microphones mounted on canal-occluding plugs near the entrance of the canal ("shallow MIRE") and using concha-mounted microphones with probe tubes extending near to the tympanic membrane and an unoccluded canal ("deep MIRE"); the latter measurements were done with the same device fitting trials as the REAT measurements. The two MIRE measurements were quite similar, with the shallow MIRE values typically better by margins of only 0.5 to $1.0 \mathrm{~dB}$ at all frequencies. A positive REAT minus MIRE difference at the lower frequencies consistent with the effects of physiological noise masking was observed. Various analyses of the data are presented and a recommendation is made for how ANR device total attenuation might be measured in a future standard as part of a hearing protector rating regime encompassing both passive and active devices.

\section{8:15}

3aNS3. Variability of field-based hearing protection device attenuation measurements. Richard Neitzel, Sebrina Somers, and Noah Seixas (Univ. of Washington, DEOHS, 4225 Roosevelt Way, NE \#100, Seattle, WA 98105)

HPD attenuation is highly dependent on individual-specific fit. Prediction of an individual's attenuation depends on measurement system accuracy and attenuation variability due to HPD refitting. This study compared attenuation measurements from two systems [one real-ear-attenuation-at-threshold (REAT) and the other microphone-in-real-ear (MIRE)] on workers using two earplugs (foam and custom-molded). Workers' perceptions of the earplugs were also evaluated. Individual's attenuation results were summarized as personal attenuation ratings (PARs). Variability in PARs from between-subject, between-day, and within-day differences was used to 
determine the lower confidence limit, or uncertainty factor (UF), of an individual's attenuation. The custom-molded earplug PARs achieved a higher percentage of labeled attenuation than did the foam earplug with both test systems, and also had higher overall acceptance among workers. MIRE PAR levels were lower than REAT levels for both earplugs, but the relationship between the two test systems was highly variable. The MIRE system had lower within-day variability than the REAT system. One individual's MIRE results were highly influential; removal of these results greatly reduced the UF for the custom-molded earplug/MIRE combination. UFs ranged from 8.8 to $13.5 \mathrm{~dB}$. These findings highlight the importance of evaluating variability in individuals' HPD attenuation, rather than relying on single measurements.

\section{$8: 35$}

3aNS4. Has the Occupational Safety and Health Administration record-keeping rule changed hearing loss prevention programs? Laurie Wells (Assoc. in Acoust., Inc., 2283 Danielle Court, Loveland, CO 80537, lwellshear@cs.com)

The Occupational Safety and Health Administration (OSHA) record-keeping rule 29 CFR part 1904.10, changed the definition of recordable hearing loss and created a separate column on the OSHA 300 form to record noise-induced hearing loss (NIHL). In an effort to identify possible effects of the record-keeping rule change, an informal written survey was distributed at the National Hearing Conservation Association (NHCA) annual conference in February, 2006. NHCA conference attendees, because they specialize in hearing loss prevention, are likely to be familiar with hearing conservation issues so their perceptions are of particular interest. A total of 36 attendees, including audiologists, audiometric technicians (OHC), industrial hygienists ( $\mathrm{IH})$, mobile test company owners, occupational medicine physicians (OMP), and occupational health nurses (OHN) completed the ten-question survey. In summary, there appears to be a consensus that the everyday practice of hearing loss prevention has been affected by the record-keeping rule change, with both positive and negative influences. Employers seem to be paying more attention to HLPPs, which is, by itself, a positive outcome. Somewhat alarming however, is the finding suggesting that recordable STSs may be being "lined-out" or "denied" inappropriately in order to keep recordable rates artificially low.

\section{8:55}

3aNS5. Hearing effects of nonoccupational sound exposure evaluated by temporary threshold shift. Hiroaki Takinami (RION Co., Ltd., 3-20-41 Higashimotomachi, Kokubunji Tokyo 185-8533 Japan, hiro@rion.co.jp) and Shoji Aono (Osaka Univ., 1-2 Yamadaoka, Suita, Osaka 565-0871, Japan)

In the 1960s, the relation between the acoustical characteristics of sound exposure and the sound-induced temporal threshold shift (TTS) of people were carefully investigated by Shoji et al. [J. Acoust Soc. Am. 48 978-987 (1970); 6th ICAA-2-8, pp. A9-A12 (1968)]. Based on these studies, an equation that estimates TTS at various test frequencies was proposed as a function of the TTS - critical-band levels. In this presentation, therelation between the sound exposure and the TTS will be reviewed, and a prototype TTS meter that estimates TTS in real time operation will be introduced. The hearing effects of some nonoccupational sounds at different places (Pachinkoparlor, disco, and livehouse) were evaluated with the TTS levels as the indicator. The sounds from portable musicplayers and music instruments of or chestra were also evaluated. The TTS levels were measured for shorttime sound exposure and compared to long - term estimates using the TTS meter. In some cases, after $2-$ or $4-\mathrm{h}$ sound exposure, the estimated TTS levels at the test frequency of $4 \mathrm{kHz}$ reached almost $20 \mathrm{~dB}$.

9:15

3aNS6. Japanese young people's recreational sound environment and hearing protection. Yôiti Suzuki and Fumitaka Saito (Res. Inst. of Electr. Commun., Tohoku Univ., Katahira 2-1-1, Aobaku, Sendai 980-8577, Japan)

Intense sound may damage hearing even if the listeners are willing to listen to it. Risk of hearing disorders due to leisure time sound is an important problem in hearing conservation. A questionnaire survey of Japanese young people's leisure time activities showed that in Japan concerts rather than discos seemed more influential and in comparison with the results in Finland, the awareness of the danger of intense sound was much less. The sound levels while listening to music with loudspeakers and at pop-music concerts were measured. While listening levels with loudspeakers were quite modest (avg.: $L_{\mathrm{Aeq}}=65 \mathrm{~dB}$ for favorite music pieces), at the two concerts the $L_{\mathrm{Aeq}}$ 's at audience positions ranged from 95 to $105 \mathrm{~dB}$. Since the pop music performed at the concerts was mild relative to typical values in the present Japanese music scenes, this level may be regarded as near the minimum that could be expected for such activities. Additionally, $L_{\text {Aeq }}$ while playing one of ten kinds of musical instruments at the player's ear was measured. The results ranged from 76 (viola) to $110 \mathrm{~dB}$ (trombone). Consideration of hearing protection at music scenes and education regarding its value seem very important.

\section{Contributed Papers}

\section{9:35}

3aNS7. Variability in real-ear attenuation at threshold evaluations of hearing protection devices. William A. Ahroon, Melinda E. Hill, and Kimberly B. Lukas (U.S. Army Aeromedical Res. Lab., Fort Rucker, AL 36362)

The American National Standard Method for the Measurement of the Real-Ear Attenuation of Hearing Protectors (ANSI S12.6-1997 [R2002]) contains explicit requirements for the physical parameters of the test facility used in measuring the performance of hearing protection devices and in subject selection and device fitting. However, the standard curiously does not provide a similar level of detail when considering the psychophysical procedure used to define "threshold" other than when an automatic recording audiometer is used. Most facilities conducting ANSIbased measurements of hearing protector performance appear to use a Bekesy tracking procedure, but other methods such as a modified method of limits or a method of adjustment procedure are implemented as well by some facilities. Following several tests of hearing protection over the past year, several examples of large variability caused by very low attenuation results in one or both runs in a few subjects have been noted. The data from these unusual subjects are analyzed and presented along with recommendations for the psychophysical procedures to be included in future versions of the standard. 
9:50

3aNS8. Comparison of passive versus active noise reduction headsets in light aircraft cockpit noise: Communications intelligibility and workload. John Casali, Brian Valimont, Jeffrey Lancaster (Auditory Systems Lab., Virginia Tech, 250 Durham Hall, Blacksburg, VA 24061, jcasali@vt.edu), and Dan Gauger (Bose Corp., Framingham, MA, 01701)

Under instrument flight rules (IFR) conditions in Cessna 172R (95 dBA) cockpit noise, eight IFR-licensed pilots flew four 3.5-h nonstop flights in an FAA-certified, single-engine flight simulator, while wearing one of four communications headsets in each flight. All flights imposed an equivalently high level of air traffic control communications, at three Speech Transmission Index (STI) values: 0.30, 0.50, and 0.70. Perceptual and psychomotor workload were also controlled. In addition to a battery of flight-related metrics (some reported elsewhere), an objective, flightembedded measure of communications intelligibility was obtained-the number of readbacks required of the pilot to attain $100 \%$ intelligibility on each command. An ANOVA revealed that as a group, the ANR headsets (Sennheiser HMEC300, Bose Aviation X, LightSPEED Thirty3G) required significantly fewer readbacks (averaging 1.2-1.3) than did the passive David Clark H-10-13.4 (averaging 1.5). Flight control performance, as reflected by deviations in assigned magnetic heading, altitude, and airspeed, was highest during flights with the Bose headset. Pilots' workload estimates were measured using the modified Cooper-Harper scale, with 1 representing lowest workload and 9 representing highest. The three ANR headsets were associated with average workload ratings of 3.4-3.5, while the passive headset yielded a higher average workload of 4.4 .

\section{0:05-10:15 Break}

\section{0:15}

3aNS9. An evaluation of the acoustic properties of an improved combat arms earplug. Mary S. Binseel and Tomasz R. Letowski (U.S. Army Res. Lab., AMSRD-ARL-HR-SD, Aberdeen Proving Ground, MD 21005, mary@arl.army.mil)

This study investigates the performance of an improved version of the combat arms earplug (CAE). U.S. Army soldiers must operate in environments which can go from quiet to noisy in a moment. They also operate in environments where the predominant noise hazard can be either continuous or impulse noise. The current earplug of choice to address these varying protection needs is the CAE. The CAE is a two-sided earplug which provides linear attenuation for steady-state noise exposure on one side and nonlinear protection for impulse noise protection on the other. Although feedback from the field has been favorable regarding the functioning of the CAE, other concerns such as fit, comfort, size, and the need to remove, turn, and reinsert the earplug to change the modality have been raised. In order to address these concerns, the Army Research Laboratory provided funding for the development of an improved CAE. The improved CAE was evaluated for attenuation of continuous and impulse noise, soldier performance in localization and speech intelligibility tasks, and soldier acceptance on issues such as ease of use, perceived protection, equipment compatibility, sizing, and comfort. Results of these experiments will be discussed.

\section{$10: 30$}

3aNS10. Design of linear time-domain filters for hearing protector modeling. Jeffrey S. Vipperman (Dept. of Mech. Eng., Univ. of Pittsburgh, 648 Benedum Hall, Pittsburgh, PA 15261) and William J. Murphy (Natl. Inst. for Occupational Safety and Health, Cincinnati, OH 45226-1998)

Accurately modeling hearing protectors for predicting the hazard underneath them is of interest to many. This project modeled the single transfer path through the hearing protector from the free field to the ear drum, as an extension to the frequency-domain modeling method presented by Price and Kalb [J. Acoust. Soc. Am. 103, 2878 (1998)]. First, the real ear attenuation at threshold (REAT) data from the hearing protectors (muff and plugs) were converted to linear quantities and curve fit to form a continuous function of frequency, while preserving the low-pass characteristic. The resulting magnitude was combined with the head-related transfer function (HRTF) to form the magnitude of the total transfer function. Using complex cepstrum, a stable, complex frequency response function (FRF) was created by assuming a minimum phase transfer path. System identification methods were used to fit the FRF data to a parametric model that was converted to an infinite impulse response (IIR) digital filter. Subject-fit REAT data were used in order to represent real-world usage. Imperfect usage was modeled by derating the subject fit data by 1 standard deviation (SD). Tests on an artificial head are conducted to evaluate the filters in the time and frequency domains. [This research was supported wholly by NIOSH.]

\section{0:45}

3aNS11. A source of high-level acoustic impulses for the measurement of hearing protectors. Jan Zera and Rafal Mlynski (Central Inst. for Labour Protection, Natl. Res. Inst., Czerniakowska 16, 00-701 Warsaw, Poland)

The paper describes a laboratory source for generating high-level, A-type acoustic impulses. The basic part of the source is a steel cylinder closed at one end with elastic foil. The blast is produced by an abrupt break of the foil caused by an increased pressure of air compressed inside the cylinder. By adjusting the air pressure inside the cylinder the impulses peak SPL may be set within a range of $150-170 \mathrm{~dB}$. As the peak SPL is increased from 150 to $170 \mathrm{~dB}$, the rise time and A duration of impulses decrease respectively from 350 to $14 \mathrm{~s}$ and from 1.1 to $0.4 \mathrm{~ms}$. An advantage of the source is that it produces impulses with highly repeatable parameters; for a given value of air pressure inside the cylinder, standard deviation of peak SPL distribution is only $1.3 \mathrm{~dB}$. The source is omnidirectional in the horizontal plane and has a nearly circular directional pattern in the vertical plane. The source is a good alternative for generating impulses with the use of explosives and is particularly useful for measurements made indoors, in the laboratory. [Work supported by the Polish Ministry of Science and Education, Grants T07B00428 and SP03.1]

\section{1:00}

3aNS12. Applicability of impulse noise hearing damage risk criteria (DRCs) to hearing protectors. Jan Zera and Rafal Mlynski (Central Inst. for Labour Protection, Natl. Res. Inst., Czerniakowska 16, 00-701 Warsaw, Poland)

Hearing damage risk criteria, DRC, concerning exposure to impulse noise, developed by CHABA [Ward CHABA Report (1968)], Pfander [J. Acoust. Soc. Am. 67, 628-633 (1980)], Smoorenburg [New Perspectives on Noise] (Raven, New York, 1982), pp. 471-490], and Dancer [Acust. Acta Acust. 3, 539-547 (1995)], have been destined for impulses encountered in open space, in free-field conditions. To adapt these criteria for conditions in which hearing protective devices (HPDs) are used, a standard procedure was proposed in the literature (e.g., by Pfander) that consists in subtracting the attenuation of the HPD, typically $25 \mathrm{~dB}$, from the impulse's peak SPL. Such a simple method does not take into account the change of the signal waveform under the HPD, usually resulting in increased $\mathrm{A}, \mathrm{C}$, or D duration. In this study, the subtraction method was verified by a comparison of impulse noise DRC with the parameters of acoustic impulses measured under the earmuff: peak SPL, rise time, and $\mathrm{A}, \mathrm{C}$, and D duration. Results show that the subtraction method underestimates the possible hearing damage risk posed by exposure to impulse noise when a hearing protector is used. [Work supported by the Polish Ministry of Science and Education, Grants T07B00428 and SP03.1].

\section{$11: 15$}

3aNS13. Performance of sound restoration hearing protection in impulsive noise. William J. Murphy (Hearing Loss Prevention Team, Natl. Inst. for Occupational Safety and Health, 4676 Columbia Pkwy., MS C-27, Cincinnati OH 45226-1998)

Hearing protection devices (HPDs) are the principal means of protecting the hearing of a person against harmful levels of noise in highly impulsive noise environments. New HPDs which utilize sound restoration circuitry were measured using a mannequin to assess the performance of 
these protectors in response to small-arms weapons fire. The results for the peak reduction and attenuation were analyzed for both indoor and outdoor measurements. The performance of the protectors exhibited little dependence with level for impulses between 150 and 170 decibels. The passive performance for a single protector characterized the active performance for these high-intensity impulses. The results were also evaluated using the US Army AHAAH cochlear model. As the peak sound-pressure level underneath the different models of protectors increased, the estimated risk of hearing loss increased. [Work supported by EPA Interagency Agreement DW75921973-01-0.]

\section{$11: 30$}

3aNS14. Criteria levels for nonoccupational noise exposure. Leslie Blomberg (Noise Pollution Clearinghouse, Box 1137, Montpelier, VT 05601)

Selecting criteria levels for nonoccupational noise exposure requires consideration of several factors. These include: (1) the need to protect public health and safety; (2) the diverse sources of nonoccupational noise exposure; (3) the inconsistent patterns of exposure, for example, the potential for multiple exposures in 1 day, or none; (4) complications caused by workplace exposure; (5) the fact that those exposed include children and minors; (6) the inability of the general public, without training or equipment, to understand traditional means of expressing noise exposure; (7) difficulties educating the public about nonoccupational noise; and (8) complications that may arise if levels are mandated or adopted by government bodies. Given these considerations, the paper argues there are actu- ally three criteria levels needed: (1) one that delimits safe noise exposure; (2) one that reflects increased levels of risk and the need for increased vigilance in protecting hearing; and (3) one that indicates unsafe exposure, for which hearing conservation should be practiced. Moreover, new and simpler ways of determining these levels are needed. Specific levels are recommended, and a method developed by the Noise Pollution Clearinghouse for use by the general public to determine if a sound is too loud is presented.

\section{$11: 45$}

3aNS15. Dosimeter measures of sound exposure experienced by university students. William A. Yost, Noorah Koita, Robert Maslo, Prasad Patel, Ruchita Patel, and Brendan Ringhouse (Parmly Hearing Inst., Loyola Univ. Chicago, 6525 N. Sheridan Rd., Chicago, IL 60626)

There is increased concern about excessive sound exposure, especially in students, resulting from the use of Ipods and similar devices. Such exposure needs to be considered in light of the daily noise dose such users experience. In this study five university students wore noise dosimeters for $10 \mathrm{~h}$ per day for 5 days, and noise levels were recorded every minute. The students also maintained a diary of their activities (activities were noted at least every half hour) during the time they wore the noise dosimeters. The diary was standardized so that each student used the same diary format. Results indicating the noise levels, noise dose, and the relationship of the levels to the students activities will be presented and discussed. In general the noise exposure experienced by these students was usually mild (less than $60 \mathrm{dBA}$ ) to moderate (between 60 and $75 \mathrm{dBA}$ ).

\section{Contributed Poster Paper}

Poster paper 3aNS16 will be on display from 7:30 a.m. to 12:00 noon. The author will be at the poster from 12:00 noon to 12:15 p.m.

3aNS16. Review of literature on hearing damage by personal stereo. Dorte Hammershøi and Rodrigo Ordoñez (Dept. of Acoust., Aalborg Univ., Fredrik Bajers Vej 7-B5, DK-9220 Aalborg Ф, Denmark, dh@acoustics.aau.dk)

In the 1980s and 1990s there was a general concern for the high levels that personal stereo systems were capable of producing. At that time no standardized method for the determination of exposure levels existed, which could have contributed to overly conservative conclusions. With the publication of ISO 11904-1:2002 and 11904-2:2004, previous studies can be viewed in a different light, and the results point, in our opinion, at levels and listening habits that are of hazard to the hearing. The present paper will review previous studies that may shed light over the levels and habits of contemporary personal stereo systems, which can produce even higher levels and are of even wider use. 


\title{
Session 3aPA
}

\section{Physical Acoustics: Cavitation I}

\author{
Claus-Dieter Ohl, Cochair \\ Univ. of Twente, Faculty of Science and Technology, P.O. Box 217, 7500 AE Enschede, The Netherlands \\ Shigeo Hayashi, Cochair \\ Univ. of Electro-Communications, 1-5-1 Chofugaoka, Chofu, Tokyo 182-8585, Japan
}

Chair's Introduction-8:30

Invited Papers

8:35

3aPA1. Attempts to observe bubble fusion in deuterated acetone. Carlos G. Camara, Seth J. Putterman, Brian Naranjo (Phys. Dept., UCLA, 2-202 Knudsen Hall, Los Angeles, CA, 90095, camara@physics.ucla.edu), Stephen D. Hopkins (Intel Corp. Portland, OR, 97205), and Kenneth S. Suslick (Univ. of Illinois, Urbana, IL 61801)

A pulsed neutron generator (PNG) and a radioactive source have been used to seed cavitation at dynamic pressures ranging up to $35 \mathrm{~atm}$. No fusion signal above background has been observed. Our upper bound is less than $1 E-4$ of the signal claimed by R. P. Taleyarkhan et al. [Phys. Rev. Lett. 96, (2006)]. Reasons for the failure of bubble fusion in acetone and future directions will be discussed. [Research funded by DARPA. We thank Brian Kappus for valuable discussions.]

\section{8:55}

3aPA2. Influence of ultrasonic frequency on sonochemical reactions. Kyuichi Yasui, Toru Tuziuti, Teruyuki Kozuka, Atsuya Towata, and Yasuo Iida (Natl. Inst. of Adv. Industrial Sci. and Technol. (AIST), Moriyama-ku, Nagoya 463-8560, Japan, k.yasui@aist.go.jp)

Numerical simulations of the pulsation of an air bubble have been performed for various ultrasonic frequencies (20 kHz to $1 \mathrm{MHz})$ and pressure amplitude taking into account the effect of nonequilibrium chemical reactions inside a bubble and that of nonequilibrium evaporation and condensation of water vapor at the bubble wall. The calculated results have revealed that as ultrasonic frequency decreases, the mole fraction of water vapor inside a bubble increases at the end of the bubble collapse. While the main oxidant created inside a vaporous bubble, which is defined as a bubble consisting mostly of water vapor at the end of the bubble collapse, is $\mathrm{OH}$ radical, that inside a gaseous bubble, which is defined as a bubble consisting mostly of noncondensable gas (air) at the end of the bubble collapse, is $\mathrm{H}_{2} \mathrm{O}_{2}$ or $\mathrm{O}$ atom when the bubble temperature is less than or more than $7000 \mathrm{~K}$, respectively. Although the optimum acoustic amplitude for the oxidant's creation inside a bubble increases as the ultrasonic frequency increases, the range of the suitable acoustic amplitude for the oxidants creation becomes wider. It possibly results in the optimum ultrasonic frequency for the oxidant's creation reported in the literatures $(200-600 \mathrm{kHz})$.

\section{9:15}

3aPA3. Comparison of sonoluminescence and water vapor plasma spectra. Masanori Sato, Nagaya Okada (Honda Electron. Co., Ltd., 20 Oyamazuka, Oiwa-cho, Toyohashi, Aichi 441-3193, Japan), Hideo Sugai, Tatsuo Ishijima, and Hirotaka Hotta (Nagoya Univ., Nagoya, Aichi 464-8603, Japan)

An experimental study was carried out to find the mechanism of $\mathrm{OH}$ spectra generation through sonoluminescence. Line spectra of $\mathrm{OH}, \mathrm{H}$, and $\mathrm{O}$ were observed very clearly in water vapor plasma generated by $2.45-\mathrm{GHz}$ microwave irradiation under the pressure of $40 \mathrm{hPa}$; however, broadband continua were not detected. Multibubble sonoluminescence (MBSL) and single-bubble sonoluminescence (SBSL) have continua, and OH spectra were detected occasionally in MBSL. However, OH spectra were not detected in SBSL and higher temperature MBSL. The sources of emission in MBSL and SBSL were considered to be identical and differences in spectra of $\mathrm{OH}$ bands were attributable to the higher temperature. Experimental results obtained using low temperature water vapor plasma confirm that $\mathrm{OH}$ spectra depend on the bubble's interior plasma temperature: that is, at low temperatures, spectra of $\mathrm{OH}(308 \mathrm{~nm}), \mathrm{Ha}$, $\mathrm{Hb}$, and $\mathrm{O}$ are clearly visible in water vapor plasma.

\section{9:35}

3aPA4. Molecular dynamics study on the nucleation and growth of cavitation bubbles. Shu Takagi (Dept. of Mech. Eng., Univ. of Tokyo, Bunkyo Ku, 7-3-1 Hongo, Tokyo 113-8656, Japan, takagi@mech.t.u-tokyo.ac.jp) and Shinichi Tsuda (Univ. of Tokyo, Tokyo 113-8656, Japan)

Molecular dynamics simulations have been conducted for the nucleation and growth processes of cavitation bubbles in a decompressed liquid. To investigate the effects of noncondensable gas, the comparison between one-component liquid argon and twocomponent liquid argon, which includes helium gas molecules as impurities, was made. As a result, we observed that a competing coarsening similar to Ostwald ripening occurs in the one-component liquid argon, while coalescence of bubble nuclei frequently 
occurs in the two-component case. In spite of the different growth patterns between the two cases, the values of the growth exponent are close to $1 / 2$ in both cases. However, it has been found that the scaling components that constitute the growth exponent, i.e., the scaling exponent for the total radius of all nuclei and that for the number of nuclei, are different between the two cases. These scaling components are discussed in relation to a driving force for bubble nuclei to grow. In addition, the asymptotic behavior of the size distribution function of bubble nuclei is also discussed using these scaling parameters.

\section{9:55-10:15 Break}

\section{Contributed Papers}

10:15

3aPA5. Frequency effects on intense sonoluminescence and bubble dynamics in sulfuric acid. Shin-ichi Hatanaka and Shigeo Hayashi (Univ. of Electro-Commun., 1-5-1 Chofugaoka, Chofu, Tokyo 182-8585 Japan, hatanaka@pc.uec.ac.jp)

Emission intensity increased remarkably with decreasing frequency for both multibubble sonoluminescence (MBSL) and single-bubble sonoluminescence (SBSL) in a concentrated sulfuric acid solution dissolving xenon gas. The spectrum of MBSL in the sulfuric acid showed the emission bands of a large peak at $388 \mathrm{~nm}$ and small peaks at 360 and $418 \mathrm{~nm}$. The increase of MBSL intensity at lower frequency was responsible for the increase of the band peak at $388 \mathrm{~nm}$ in addition to the increase in continuous spectrum. The bubble dynamics in the sulfuric acid was different from that in water. The intense SBSL was emitted when the bubble was moving on an ellipselike trajectory. The intense MBSL was emitted even when multiple bubbles formed bubble clusters, which could not emit light in the water case.

\section{0:30}

3aPA6. Experimental analysis of blackbody emission from sonoluminescence in sulfuric acid. Stephen D. Hopkins, Carlos G. Camara, and Seth J. Putterman (Phys. Dept., UCLA, 2-202 Knudsen Hall, Los Angeles, CA 90095)

The spectrum of emission from a single xenon bubble in concentrated sulfuric acid driven at $30 \mathrm{kHz}$ is an excellent fit to Planck's law with a surface temperature of $8000 \mathrm{~K}$. The measured flash width and emission radius are also consistent with blackbody emission. In this study the only fitting parameter available is the temperature [Phys. Rev. Lett. 95, (2005)]. [Research funded by DARPA.]

\section{0:45}

3aPA7. Drop tube generates 10-W flashes of sonoluminescence. Brian A. Kappus, Avic Chakravarty, ${ }^{a}$, and Seth J. Putterman (Phys. Dept., UCLA, 1-129 Knudsen Hall, Los Angeles, CA 90095)

Use of a low vapor pressure liquid such as phosphoric acid, with dissolved xenon in a vertically exited tube, generates $\sim 200$-ns flashes of sonoluminescence with a peak power of $10 \mathrm{~W}$. We are in the process of characterizing the bubble motion by use of backlighting, stroboscopic, and streak photography. We will also broach the topic of disequilibrium between atom and electron temperatures. [Research funded by DARPA. We thank Carlos Camara and Shahzad Khalid for valuable discussions.] ${ }^{a)}$ Deceased.

\section{1:00}

3aPA8. Attempts to observe sonoluminescence from a single bubble driven at $10 \mathrm{MHz}$. Shahzad Khalid, Carlos G. Camara, and Seth J. Putterman (Phys. Dept., UCLA, 2-202 Knudsen Hall, Los Angeles, CA 90095)

A 10-MHz spherical transducer array is used to generate cavitation at its focus with a field of $\sim 100 \mathrm{~atm}$. Sonoluminescence as well as lightscattering measurements will be discussed. [Research funded by DARPA.]
11:15

3aPA9. Unidentified energy conversions in an oscillating bubble. Karel Vokurka (Phys. Dept., Tech. Univ. of Liberec, 46117 Liberec, Czech Republic) and Silvano Buogo (CNR IDAC, 00133 Roma, Italy)

Acoustic radiation of freely oscillating bubbles has been studied experimentally. The oscillating bubbles have been generated by underwater spark discharges. Recorded pressure waves made it possible to determine the acoustic energy radiated by the oscillating bubbles and the potential energy of the bubbles when attaining the first and second maximum volumes. Comparison of the potential and acoustic energies revealed that about $70 \%$ of the potential energy of the bubble available at the first maximum volume has been converted into an unidentified energy. A possible candidate for the energy conversion mechanism seems to be internal converging shock wave in the bubble interior. The presence of this wave can be observed indirectly in a radiated pressure wave. The measured energies are compared with energies computed using a suitable model. This comparison shows that the theoretical values agree with experimental data until the bubble attains the first minimum volume. However, at later times discrepancies are observed. [Work has been partly supported (K.V.) by the Czech Ministry of Education as the research project MSM 46747878501.]

\section{1:30}

3aPA10. Optical observation of collapses of two bubbles adhered to the quartz wall under ultrasound irradiation with high-speed video camera: Micro-jet arising from two collapsing bubbles. Kenji Yoshida, Shintaro Nakatani, and Yoshiaki Watanabe (Faculty of Eng., Doshisha Univ., 1-3 Tataramiyakotani, Kyotanabe, Kyoto, Japan, etf1103@mail4.doshisha.ac.jp)

The mechanical action of a micro-jet, which arises from the collapse of bubbles, has been widely used for ultrasonic cleaning and sonoporation in medical field. The generation mechanism of a micro-jet induced by the ultrasound irradiation is focused on. In previous theoretical and experimental researches, the generation mechanism is well known when a single bubble is adhered to a rigid wall. However, its mechanism is not understood in detail when multiple bubbles exist on rigid wall. The optical observation of multi-bubble collapse greatly contributes to the development of micro-jet applications. It was observed that the collapses of two bubbles adhered to the quartz wall in an ultrasound field with high speed video camera (HPV-1, SHIMADZU). Maximum recording rate of this high speed video camera is 1000000 frames/second. The emitted ultrasound frequency is $27 \mathrm{kHz}$. From the observation, one of two bubbles was deformed after these bubbles vibrate. The micro-jet was generated to the opposite side against the deformed bubble when the deformed one touches the other. Then, the direction of the micro-jet to the quartz wall was almost horizontal. The number of micro-jets increased every time the distorted bubble touched the other. Finally, five micro-jets to the various directions were observed at the same instant. 


\title{
Session 3aPP
}

\section{Psychological and Physiological Acoustics and Musical Acoustics: Perception of Music and Speech: Evidence for Modularity and for Common Processing Mechanisms}

\author{
Diana Deutsch, Cochair \\ Univ. of California, San Diego, Dept. of Psychology, La Jolla, CA 92093 \\ Yoshitaka Nakajima, Cochair \\ Kyushu Univ., Dept. of Acoustic Design, 4-9-1 Shiobaru, Minami-ku, Fukuoka 815-8540, Japan
}

Chair's Introduction-8:30

Invited Papers

8:35

3aPP1. Speech perception: Development from general mechanisms to modules. Patricia K. Kuhl (Inst. for Learning \& Brain Sci. and Dept. of Speech and Hearing Sci., Univ. of Washington, Seattle, WA 98195)

Are the mechanisms subserving speech perception modular or general process in nature? I will adopt a developmental perspective to address this question. Using data from cross-cultural, cross-species, and developmental experiments, I will argue that, early in infancy, the mechanisms underlying speech perception are domain general and rely on processing that is not exclusive to speech signals. Language evolved to capitalize on general mechanisms of perception and action, and this ensures that all infants, regardless of the culture in which they are born, initially respond to and learn from exposure to language. As experience with language ensues, neural commitment to the properties of the language to which an infant is exposed results in dedicated neural networks and speech perception becomes more specialized. Finally, I will suggest that adult members of the culture help instill language in the child by adjusting the language they use when addressing infants and children (motherese), a speech style that exhibits interesting acoustic properties. Language input to the child enhances its learnability. A model of the developmental process will be described. [Research supported by NSF and NIH.]

\section{$8: 55$}

3aPP2. Auditory events in language and music. Yoshitaka Nakajima and Kazuo Ueda (Faculty of Design, Kyushu Univ., 4-9-1 Shiobaru, Minami-ku, Fukuoka 815-8540, Japan, nakajima@design.kyushu-u.ac.jp)

Perceptual organization of both language and music is based on temporal units that can be called auditory events. Speech syllables and musical notes correspond typically to auditory events. The listener categorizes these events in various ways in order to extract information. We are at a preliminary stage of searching for acoustic cues that the auditory system can utilize to determine auditory events. Averaged intensity of a speech signal within a moving Gaussian temporal window of $15 \mathrm{~ms}$ gives cues of syllable boundaries, especially when this averaged intensity is compared with the intensity change obtained with a Gaussian temporal window of $200 \mathrm{~ms}$. The change of intensity obtained through a filter of about $600-2200 \mathrm{~Hz}$ compared with the intensity change obtained through an almost flat filter also gives cues of syllable boundaries. Thus, intensity cues and spectral cues both work to mark syllables. This holds both in Japanese and in English. Such intensity cues may work when applied to monophonic or homophonic music, but spectral changes do not correspond clearly to musical notes. Thus, the perceptual mechanisms that determine speech syllables and musical notes can be different. [Work supported by JSPS and 21st Century COE, Kyushu University.]

\section{9:15}

3aPP3. Music and language in deaf children with cochlear implants. Takayuki Nakata, Sandra E. Trehub, Yukihiko Kanda, and Haruo Takahashi (Dept of Psych., Nagasaki Junshin Catholic Univ., 235 Mitsuyama-machi, Nagasaki-shi, Nagasaki-ken 852-8558, Japan, Dept. of Psych., Univ. of Toronto, Nagasaki Bell Hearing Ctr., Nagasaki Univ. Grad School of Biomed. Sci.)

Congenitally deaf children with cochlear implants successfully perceive speech in quiet environments, and many produce intelligible speech with normal voice quality. Because implants were designed to optimize speech rather than music perception, timing cues are preserved at the expense of pitch cues. Consequently, adult implant users tend to discontinue musical activities that were enjoyable prior to the onset of deafness. By contrast, congenitally deaf children enjoy a variety of musical activities including listening, singing, and playing instruments. Research in our laboratory suggests that musical activities may enhance child implant users' speech perception skills. For example, initiation of music listening at home by children with cochlear implants predicted their success on a word recognition task. Moreover, earlier ages of implantation were positively associated with enhanced motivation for listening to music. In addition, child implant users were able to identify musical themes that accompanied their favorite television programs. Unlike hearing children, however, they could not recognize the music from the melody (i.e., pitch patterning) alone. Analyses of these children's singing revealed rhythmic patterning similar to that of hearing children but pitch patterning that was grossly distorted. In short, rhythm plays a central role in child implant users' musical experience. 
3aPP4. Dissociation of music and speech: Evidence from statistical learning in congenital amusia. Isabelle Peretz (Dept. of Psych., Univ. of Montreal, B.O. 6128, Succ. Ctr. ville, Montreal, QC, Canada H3C 3J7, isabelle.peretz@umontreal.ca) and Jenny Saffran (Univ. of Wisconsin-Madison, Madison, WI 53706)

Individuals who suffer from congenital amusia (commonly called tone-deafness) have experienced life-long difficulties in processing of music. The deficit can be traced down to an impairment in fine-grained pitch discrimination [Hyde and Peretz, Psychol. Sci., (2004)]. Their language abilities appear intact. One possible account for this dissociation between music and speech is that amusics lack normal exposure to music because of their lifelong history of musical failures. In other words, amusics might be able to acquire basic musical abilities if given appropriate exposure. To test this possibility, a group of 11 adults with congenital amusia, and their matched controls, were exposed to a continuous stream of syllables or tones for at least $21 \mathrm{~min}$. Their task was to try to identify words or motifs, defined by transitional probabilities, following the procedure used by Saffran and collaborators [Cognition, (1999) (2001)]. The results show that amusics can learn novel words as easily as controls whereas they systematically fail on musical materials. Thus, limited exposure cannot fully account for the musical disorder. Implications for the domain specificity/generality debate of statistical learning will be discussed.

\section{9:55}

3aPP5. Nonlinguistic rhythm perception depends on culture and reflects the rhythms of speech: Evidence from English and Japanese. Aniruddh D. Patel, John R. Iversen (The Neurosci. Inst., 10640 John Jay Hopkins Dr., San Diego, CA 92121), and Kengo Ohgushi (Kyoto City Univ. of Arts, Kyoto, Japan)

Does a listener's native language influence the perception of nonverbal rhythms? One way to address this question is to examine the perception of rhythmic grouping, as suggested by Jakobson, Fant, and Halle in Preliminaries to Speech Analysis. Grouping refers to the perceptual clustering of events into higher level units such as phrases. Several principles of grouping of auditory patterns, established a century ago, have come to be thought of as innate properties of perception. The supporting data have come entirely from Western cultures, however. In this study, native English and Japanese speakers were tested for their perception of grouping in simple rhythmic sequences of tones. Members of the two cultures showed different patterns of perceptual grouping, demonstrating that basic auditory processes are shaped by culture. Furthermore, measurements of the temporal structure of English versus Japanese speech and music suggest that the observed differences reflect the rhythms of the two languages, rather than rhythmic differences between American and Japanese music. While it has long been known that a listener's native language influences the perception of speech, these results suggest that native language can also exert an influence on general auditory perception at a basic level. [Supported by Neurosciences Research Foundation and by JSPS.]

10:15-10:30 Break

\section{0:30}

3aPP6. Two issues at the interface between speech and music. Diana Deutsch (Dept. of Psych., Univ. of California, San Diego, La Jolla, CA 92093) and Trevor Henthorn (Univ. of California, San Diego, La Jolla, CA 92093)

In what ways are speech and music subserved by domain-specific mechanisms, and in what ways by common neural substrates? This issue is addressed with respect to absolute pitch, which has generally been considered to be a musical faculty accessible only to rare individuals. Based on several findings obtained from speakers of tone languages, it is argued that absolute pitch originally evolved to subserve speech, and that it may be acquired readily during the first year of life, if infants are given the opportunity to associate pitches with verbal labels. It is further conjectured, based on other findings, that the early acquisition of pitch accent languages, such as the Tokyo and Osaka dialects of Japanese and the Hamkyeng and Kyengsang dialects of Korean, might also be conducive to the acquisition of absolute pitch. Perceptual relationships between speech and song are next considered. These generally differ in their features; however, a spoken phrase can be perceptually transformed to appear as song rather than speech, simply by repeating it several times over without any alteration in the signal (Deutsch, 2003). This phenomenon is demonstrated, its parameters are explored, and its implications for the mechanisms underlying perception of speech and song are discussed.

\section{Contributed Papers}

\section{0:50}

3aPP7. Training musicians and nonmusicians to discriminate Mandarin tones. Terry L. Gottfried and Grace Y.-H. Ouyang (Dept. of Psych., Lawrence Univ., Appleton, WI 54912-0599, rewgottt@lawrence.edu)

Musicians and nonmusicians were compared in their ability to learn Mandarin tone contrasts. Participants were pretested to assess their accuracy in tone discrimination. Some were then given six sessions of articulatory and acoustic training over 3 weeks; others were given no training. At the end of 3 weeks, participants were again tested on their discrimination accuracy. Listeners judged whether the tones (tone 1, high level; tone 2, mid rising; tone 3, low dipping; tone 4 , high falling) of two different
Mandarin syllables were the same or different. In some trials, listeners discriminated tones in words spoken by two speakers; in other trials, they discriminated tones in words spoken by one speaker only. The pretest revealed that musicians are significantly more accurate in discriminating Mandarin tones than nonmusicians. Musicians and nonmusicians who received the training regimen performed more accurately than untrained listeners on the posttest discrimination. Musicians showed significantly less improvement than nonmusicians with training, probably due to ceiling effects (their posttest accuracy was over 95\%). Two-speaker discrimination trials were significantly more difficult for all listeners, but especially for nonmusicians. Discrimination of tones 2 and 3 was most difficult for all listeners, but showed considerable improvement after training. 
3aPP8. The effects of musical experience on linguistic pitch perception: A comparison of Norwegian professional singers and instrumentalists. Maren Helene R $\varnothing$, Dawn Behne (Dept. of Psych., Norwegian Univ. of Sci. and Technol., N-7491 Trondheim, marenhel@stud.ntnu.no), and Yue Wang (Simon Fraser Univ.)

Speech prosody and music share tonal attributes well suited for studying cross-domain transfer effects. The present study investigates whether the specific pitch experience acquired by professional singers and instrumentalists transfers to the perception of corresponding prosodic elements in a native and non-native language. Norwegian and Mandarin words with tonal distinctions, together with corresponding hummed tones, were presented dichotically in a forced attention listening test to three groups of native Norwegian listeners: professional singers, professional instrumentalists, and nonmusicians. While instrumentalists and singers were both more accurate (higher percent correct for both ears) than nonmusicians for Mandarin linguistic and hummed tones, only instrumentalists showed positive transfer to corresponding native Norwegian stimuli. Results indicate a pattern of perceiving tonal distinctions that mirrors the pitch experience acquired through professional vocal and instrumental training: Instrumentalists generally appear to rely on an autonomous, categorical pitch strategy, consistent with their primary training with discrete, fixed intervals. Singers, on the other hand, tend to use a similar strategy for nonlinguistic pitch perception, but not in processing native tonal contrasts. Overall, pitch experience acquired through musical training appears to have a positive transfer to non-native speech perception.
3aPP9. Effects of musical experience on linguistic pitch perception training. Dawn Behne (Psych., Norwegian Univ. of Sci. and Technol., 7491 Trondheim, Norway), Yue Wang (Simon Fraser Univ., Burnaby, BC V5A 1S6, Canada), Maren Helene Rø, Ann-Karin Hoff, Hans Andreas Knutsen, and Marianne Schmidt (Norwegian Univ. of Sci. and Technol., 7491 Trondheim, Norway)

Research continues to address the extent to which music ability transfers to other tasks and processes, among them speech perception. The current study examines the transfer of music experience to native and non-native linguistic pitch perception and tracks this process during training. Participants were nonmusicians (NMs) and music conservatory students (CMs), all of whom were native Norwegian listeners. Participants were tested with Norwegian and Mandarin materials in a tone-based and intonation-based directed attention dichotic listening task, administered five times during 2 weeks. Results show that training generally leads to increased accuracy in linguistic pitch perception. CMs are more accurate than NM in both tasks, and maintain this advantage throughout training. For both groups, training led to a decreased difference in performance between ears, with notably different patterns of ear improvement between NMs and CMs. Across languages, whereas CMs had improved accuracy in both tasks, NM showed no improvement in the tone task for either language. Findings indicate that musically trained listeners' experience positively transfers to native and non-native linguistic pitch perception, an advantage which, in training, affords them more extensive improvement across linguistic pitch tasks and in particular for a non-native pitch task where the native linguistic system does not interfere.

11:35-11:55

Panel Discussion

THURSDAY MORNING, 30 NOVEMBER 2006

MOLOKAI ROOM, 8:00 A.M. TO 12:00 NOON

Session 3aSC

\title{
Speech Communication and Psychological and Physiological Acoustics: Second Language Acquisition II (Poster Session)
}

\author{
Sang Yee Cheon, Cochair \\ Univ. of Hawaii, Dept. of East Asian Languages and Literature, Honolulu, HI 96822
}

Nobuaki Minematsu, Cochair

Univ. of Tokyo, Dept. of Frontier Informatics, 5-1-5 Kashiwanowa, Kashiwa-shi, Chiba 277-8562, Japan

\section{Contributed Papers}

\begin{abstract}
All posters will be on display from 8:00 a.m. and 12:00 noon. To allow contributors an opportunity to see other posters, contributors of odd-numbered papers will be at their posters from 8:00 a.m. to 10:00 a.m. and contributors of even-numbered papers will be at their posters from 10:00 a.m. and 12:00 noon.
\end{abstract}

3aSC1. A computer-assisted instruction system for self-teaching of discriminating Chinese tones based on their acoustical properties. Shizuo Hiki, Kazuko Sunaoka, and Song Liu (Waseda Univ., 2-579-15 Mikajima, Tokorozawa, 359-1192 Japan, hiki@waseda.jp)

A computer-assisted instruction (CAI) system for self-teaching to discriminate Chinese tones was developed, and provided through the internet to Japanese college students of Chinese language class. The data of acoustical analysis and perceptual judgment on the Chinese tones played an important role in designing the system. The result of observation on characteristic errors in discriminating Chinese tones by the Japanese students was also utilized. This system offered three kinds of use for self-teaching: testing, training, and reviewing. The original word lists consisted of 15 bisyllabic words with every combination of the four tones of Standard Chinese, involving different rates of unvoiced consonants, which disturbed the tone discrimination. In the partial word lists, only words having tone 2 and/or tone 3 , which were difficult for the beginners, were excerpted from the original lists. Their speech samples with both female and male voices were accommodated. Temporal changes in fundamental frequency were extracted from the speech samples and displayed as visual cues in the training. It was also possible in the training to present only the words answered incorrectly in the preceding testing. By selecting suitable uses and word lists with the progress of self-teaching, the required time for achieving the goal was reduced. 
3aSC2. Providing new methods for teaching Japanese speech communication for learners of Japanese as L2: Developing teaching materials using MRI-movies and natural speech data. Ryoko Hayashi, Chunyue Zhu, Toshiyuki Sadanobu, Jumpei Kaneda (Grad. School of Cultural Studies and Human Sci., Kobe Univ., Nada-ku Tsurukabuto 1-2-1, Kobe 657-8501, Japan, rhayashi@kobe-u.ac.jp), Donna Erickson (Showa Music Univ., Kanagawa 243-8521, Japan), Nick Campbell (ATR, Hikaridai 2-2-2, 619-0288, Japan), and Miyoko Sugito (Inst. for Speech Commun. Res., Nara 631-0014, Japan)

It is critical to provide people learning Japanese as a second language information about natural speech, since there is wide variability of articulation and speaking styles associated with various social attitudes and/or expressions in Japanese. For developing teaching materials for English and Chinese learners of Japanese as a second language, two new methods are demonstrated. One utilizes MRI-movies that dynamically demonstrate differences among vowel articulations in Japanese, English, and Chinese. This approach is effective for teaching good pronunciation, especially with regard to consonant cluster production, since the timing of the articulation for consonant clusters is visibly presented. The other is audio-visual data of natural speech in Japanese that demonstrate several typical expressions, e.g., wry face and strained (laryngealized) voice for asking favors politely. This type of material shows not only variations of speech communication in Japanese but also cultural differences among native speakers of Japanese, English, and Chinese. [Work supported by MEXT.]

3aSC3. Effects of training with sentences at varied speaking rates on native English speakers' perception of Japanese vowel length. Yukari Hirata, Emily Cullings, Elizabeth Whitehurst, Jacob Whiton (Dept. of East Asian Lang. and Lit., Colgate Univ., 13 Oak Dr., Hamilton, NY 13346), and Stephen Lambacher, G. (Univ. of Aizu, Aizu-Wakamatsu, Fukushima 965-8580, Japan)

In training non-native speakers to perceive difficult second language (L2) phonetic contrasts, providing variation in speakers and phonetic contexts helps the formation of L2 categories [Pisoni and Lively, in Speech Perception and Linguistic Experience (1995), pp. 433-459. The present study tested the hypothesis that high stimulus variability in speaking rate would have positive effects for L2 learners. Native English speakers' ability to identify Japanese vowel length was examined with three types of training: slow-only, fast-only, and slow-fast. Their perceptual abilities were measured with a pretest and posttest and compared with a control group that was not trained. Test stimuli were sentences containing Japanese disyllables spoken at three rates (slow, normal, fast). Participants were trained to identify the length of the second vowel of disyllables embedded in a sentence and they received immediate feedback. The pretest to posttest improvement of the slow-only $(8.2 \%)$ and slow-fast $(8.7 \%)$ training groups was significantly greater than the control group's improvement (3.9\%). However, the fast-only $(7.7 \%)$ training group's improvement did not significantly differ from the control group's improvement. In addition, slow-fast training, unlike other training, had an advantage in performance on the fast rate stimuli. Implications for the variability theory will be discussed. [ Work supported by NSF Grant No. BCS0418246.]

3aSC4. Factors affecting the success of non-native vowel perception training. Kanae Nishi and Diane Kewley-Port (Indiana Univ., 200 S. Jordan Ave., Bloomington, IN 47405)

Previously, Nishi and Kewley-Port [J. Acoust. Soc. Am. 118, 1900 (2005)] reported the successful outcomes of an intensive vowel perception training protocol for Japanese and Korean learners of English. Their results indicated that vowel training worked best when large numbers of vowels in nonsense words were presented within the training blocks. In this report, additional analyses were performed in order to explore the generalization of training from nonsense to real words that were presented only during pre- and post-training tests. Results showed that, although both groups showed steady and substantial improvement for all vowels in nonsense words, generalization to real words was inconsistent across lis- tener groups, different vowels, and talkers. These results indicated that: (1) native vowel phonology had a considerable influence on the success of our structured, intensive identification training protocol using many vowels; (2) nonsense word training incorporating high natural variability across phonetic contexts and talkers may not be sufficient to achieve the desired generalization to real words. These results are explained in part by the vowel acoustics (formant frequencies and vocalic duration) of the stimulus materials used for training and tests. [Work supported by NIDCD-006313 and 02229.]

3aSC5. An analysis of the factors reading rate, vocabulary ability, and speaking proficiency in relation to the test of English for international communication (TOEIC) scores of Japanese learners of English. Keiko Asano and Michiko Mochizuki Sudo (Juntendo Univ., 1-1 Hiragagakuendai Inbamura, Inbagun, Chiba 270-1695, Japan, keiko_asano@sakura.juntendo.ac.jp)

This study investigated the relationships between the TOEIC scores and the two factors of the reading rates and vocabulary abilities of Japanese learners of English. Also studied are the relationships between the scores of the two TOEIC sections and the learners' speaking proficiencies. Applying the Rauding theory, which reported the same comprehension processes underlying reading and listening, to second language acquisition, this study tested the hypothesis that the processing time for reading had an effect on that for listening. The Japanese subjects in this study took TOEIC and standardized tests of reading rates and vocabulary abilities, and also participated in a production experiment. The results showed high correlations between the TOEIC scores and the two factors of reading rates and vocabulary abilities. They also showed the tendency that the improvement of reading rates and vocabulary abilities contributed to the improvement of not only the scores of the TOEIC Reading Section, but also those of the TOEIC Listening Section. In addition, this study clarified the degree to which the speaking proficiency measured by naturalness judgments of native speakers of English corresponded to the proficiency indirectly measured by the TOEIC Listening Section.

3aSC6. The long-term retention of phonetic categories by Japanese immersion graduates. Tetsuo Harada (School of Education, Waseda Univ., 1-6-1 Nishi Waseda, Shinjuku, Tokyo 169-8050, Japan)

English-speaking children in a Japanese immersion program, in which many content subjects are taught in Japanese, were reported to be successful in establishing phonetic categories for single and geminate stops in Japanese, although their categories deviated from those of Japanese monolinguals. However, it is unknown to what extent those children can retain their phonetic categories after they exit the program. Ten participants who had attended a Japanese immersion program in the United States from kindergarten or grade 1 to grade 5 and who were taking a university-level intermediate or advanced Japanese course at the time of data collection were recorded producing Japanese words including both singletons and geminates. Mean closure duration values were obtained, and the ratio of geminates to singletons for the immersion graduates was compared with that of English learners of Japanese having the same proficiency level in a regular Japanese program. The results show that the immersion graduates, who had been exposed to a substantial amount of Japanese at an early age, still retained the phonetic categories and could contrast singletons with geminates in Japanese, although their closure duration tended to be longer than the norm of monolingual Japanese speakers.

3aSC7. The effects of training context on perceptual learning of accented speech. Jessica E. Duke and Sabrina K. Sidaras (Dept. of Psych., Emory Univ., Atlanta, GA 30322)

This study investigates factors influencing adult listeners' perceptual learning of talker-specific and accent-general properties of spoken language. Exposure to foreign accented speech under different training con- 
ditions was used to examine mechanisms involved in perceptual learning. Adult native speakers of American English were exposed to English words produced by six native-Spanish-speaking adults. During training, the Spanish-accented words were either grouped by speaker, by word, or were randomized with respect to speaker and word. The different types of training were hypothesized to direct attention to particular characteristics of the accented speech. At test, listeners were asked to transcribe novel words produced by familiar or unfamiliar accented speakers. Regardless of training condition, listeners who had been exposed to Spanish-accented speech during training were more accurate at test than an untrained control group. However, high variability training in which words were randomized led to better transcription performance at test than training with items grouped by speaker or by word. These findings suggest that high variability training may direct attention to the accent-general properties of speech necessary for perceptual learning of accented speech.

3aSC8. Development and validation of an automatic spoken Spanish test. Nicole Wilson, Elizabeth Rosenfeld, and Jared Bernstein (Ordinate Corp., 800 El Camino Real, Menlo Park, CA 94025)

The development and validation of a fully automatic computer-based test of spoken Spanish is described. The test takes between 15 and 20 min to complete and comprises readings, elicited imitations, opposites, shortanswer questions, sentence constructions, and story retellings. The only response modality is spoken Spanish. Sets of vetted items were assembled into tests presented to over 1000 adult non-native Spanish learners and over 1000 native Spanish speakers from several countries. Expert human judgments of the non-native responses showed that the spoken response material carried sufficient information for highly reliable judgments of proficiency. In the development and validation process, over 60000 responses were transcribed and over 21000 human judgments were analyzed. The automatic scoring system is described. Its validation includes comparisons of machine scores with interactive human interviews and human ratings of speech recordings. Results suggest that machine scores have virtually the same information that is found in Oral Proficiency Interviews conducted by the U.S. Government raters or by official ACTFL interviews. Correlation between machine scores and combined interview scores $(r=0.92)$ is higher than the interrater reliability of the professional interviewers themselves.

3aSC9. French conversations do not show evidence of phonetic accommodation to a non-native interlocutor. Caroline Smith (Dept. of Linguist., Univ. of New Mexico, MSC 03 2130, 1 Univ. of New Mexico, Albuquerque, NM 87131-1196)

Although a speech style known as foreigner talk has been described, speech addressed to a reasonably fluent non-native interlocutor may show little or no accommodation by native speakers. This issue is of interest in understanding whether on-line modifications to speech are an important contributor to phonetic variation. Ten native speakers of Parisian French were recorded individually as they performed map tasks, one with another native speaker of French, and another with an American who identified himself as such at the beginning of the conversation, but who spoke French during the task. Previous work has found that French listeners who heard brief extracts from these conversations could not reliably identify which interlocutor was involved. In an attempt to identify other possible differences in speech to natives and non-natives, two prosodic characteristics of the conversations were analyzed, vowel devoicing and the epenthesis of word-final schwas. The hypothesis was that vowel devoicing, which is stylistically marked, would be disfavored in speech to nonnatives, while schwa epenthesis, characteristic of a deliberate speech style, would be more frequent. The speakers actually showed similar patterns with both interlocutors, suggesting that variation in these properties is not conditioned by the linguistic status of the interlocutor.
3aSC10. Acoustic correlates of accentedness and intelligibility of Spanish-accented English vowels. Lynne C. Nygaard, Sabrina K. Sidaras, Jessica E. Duke, and Stig T. Rasmussen (Dept. of Psych., Emory Univ., Atlanta, GA 30322)

The present study investigated the acoustic characteristics of English vowels produced by native speakers of Spanish and the relationship between the acoustic properties of non-native vowel production and perceived accentedness and intelligibility. Twelve native speakers of Spanish were recorded producing monosyllabic words in list format. Individual vowel tokens from a variety of consonant environments were identified and acoustic analyses were conducted, including measures of duration and formant (F1, F2, F3) frequency. Listener ratings of accentedness and intelligibility were obtained from a separate set of sentence-length utterances. The analyses showed that average overall vowel duration as well as the duration of individual vowels was positively correlated with judgments of accentedness and negatively correlated with overall sentence intelligibility. Speakers who were judged to be more accented and less intelligible produced vowels of longer duration than speakers who were judged to be less accented and more intelligible. Analyses of spectral characteristics and comparisons to native English speakers' vowel productions will also be reported. These findings suggest that the temporal properties are one aspect of non-native vowel productions that significantly contribute to the perception of accentedness as well as to the overall intelligibility of individual native Spanish speakers.

3aSC11. Factors influencing L2 vowel perception and production. Karece Lopez (Dept. of Speech, Commun. Sci., \& Theatre, St. Johns Univ., 8000 Utopia Pkwy, Jamaica, NY 11439), Yan Helen Yu (CUNY Grad. School, New York, NY), and Fredericka Bell-Berti (St. Johns Univ., Jamaica, NY 11439)

This study investigates relations among factors expected to influence vowel perception and production in second language learning, including AoA, LoR, L2 education (before coming to and in the US), and language use (in the US). Vowel production will be examined through phonetic transcription, listener identification, and acoustical analysis. Preliminary evidence indicates that, even though our participants arrived in the US after age 12 , AoA is still of critical importance in second language learning. Furthermore, and as expected, LoR also contributes to L2 proficiency. AoA and LoR, however, do not account for the ability of all the participants to identify and produce the vowels of English; thus, we must also consider other factors, including L2 education and use. The hypothesis is that L2 educational background and language use influence L2 English speakers' perception and production of English vowels.

3aSC12. Production of English stressed vowels and interstress intervals within and between words in first and second language acquisition. Michiko M. Sudo and Ikuyo Kaneko (School of Health and Sports Sci., Juntendo Univ., 1-1 Hiragagakuendai, Inbamura, Inbagun, Chiba, 270-1695, Japan, mmsudo@polka.plala.or.jp)

The purpose of this study was to observe the differences between first and second language acquisition in English durational patterns with respect to interstress intervals (ISIs). Production experiments were carried out to investigate the durational patterns used by three groups of speakers: adult native speakers of American English, American third graders, and Japanese learners of English. Two sets of English sentences were devised as the linguistic material, and the sentences in each set differed in the number of nominally unstressed syllables that intervened between a target stressed syllable and the next stressed syllable. These sets of sentences contained two different target stressed vowels, and the ISIs within and between words. The shortest ISIs were produced by adult American speakers regardless of the number of unstressd syllables in the target ISIs. The Japanese learners produced shorter ISIs than the American children in one set of sentences, while in the other set, the American children produced 
shorter ISIs than the Japanese. Regarding the durations of target stressed vowels, foot-level shortening was observed in the three subject groups, the American children exhibiting the highest percentage of shortening followed by the American adults and then the Japanese.

3aSC13. Production and perception of American English vowels by native Japanese speakers. Takeshi Nozawa (Ritsumeikan Univ., 1-1-1 Nojihigashi, Kusatsu, Shiga, 525-8577 Japan), Elaina M. Frieda (Auburn Univ., Auburn, AL 36849), and Ratree Wayland (Univ. of Florida, Gainesville, FL 32611-5454)

Native speakers of Japanese (NJ) produced American English vowels in $/ \mathrm{CVC} /$ contexts. Twelve native speakers of American English listened to and identified the vowels in the Japanese speakers' productions. In general, the vowels difficult for the NJ subjects to disambiguate in production were also difficult for them to differentiate in perception. An acoustic analysis revealed that the $\mathrm{NJ}$ speakers' attempted /i/ and /I/ tokens and /a/ and $/ \mathrm{\gamma} /$ tokens largely overlapped in the vowel space, but significant differences in duration were observed between their /i/ and /I/ tokens. These findings agree with the results of our previous studies. In our perception experiment, $/ \mathrm{a} /-I_{\mathrm{N} /}$ was the most difficult vowel contrast for the same group of $\mathrm{NJ}$ speakers to discriminate, and longer /i/ tokens were more correctly identified and shorter /i/ tokens were misidentified more often as $/ \mathrm{I} /$. In contrast to the perception experiment, the intelligibility of American English vowels produced by the NJ subjects was not affected by the place of articulation of the preceding consonant or by the voicing status of the following consonant. However, the American English vowels produced by the $\mathrm{NJ}$ subjects were less intelligible when a liquid /l/ follows, which may be attributed to their inability to produce /1/ authentically.

3aSC14. Durational characteristics in production of English consonant sequences by Japanese speakers. Kuniyoshi Tanaka (Grad. School of Humanities, Hosei Univ., 2-15-2 Ichigayatamachi, Shinjuku-ku, Tokyo 162-0843, Japan, tanaka22@abox.so-net.ne.jp) and Keiichi Tajima (Hosei Univ., Chiyoda-ku, Tokyo 102-8160, Japan)

Japanese has more limited consonant sequences (e.g., "honda," "nissan") than English (e.g., "script"). This difference becomes a problem for Japanese learners of English when they produce English words that contain consonant sequences. Japanese speakers often avoid these sequences by inserting epenthetic vowels between consonants or following wordfinal consonants (e.g., English "script" as/su.ku.ri.pu.to/). The present study examines how Japanese speakers produce consonant sequences in English and how speaking rate affects their productions. A group of native Japanese speakers and a control group of native English speakers read English words at normal and slow speaking rates. Segment durations in target consonant sequences were measured, and ratios of segment to word duration and segment to consonant sequence duration were calculated. Preliminary results suggest that the segment durations and ratios are clearly different between the two groups of speakers. For example, in /s/ + consonant sequences, Japanese speakers tend to produce a relatively shorter/s/ and a longer consonant compared to native English speakers. Also, the duration ratios do not seem to vary depending on the speaking rate for either group of speakers. Results will be discussed in terms of timing control in second-language learning. [Work supported by MEXT.]

3aSC15. The speech learning model: English and Japanese voice onset time in mature bilingual talkers. Timothy J. Riney (Intl. Christian Univ., Tokyo, 181-8585, Japan)

With reference to the Speech Learning Model (Flege, 1995 version), this study looked for evidence among mature American and Japanese bilinguals that (a) L1 VOT changed over time and (b) despite minimal L1-L2 phonetic differences, L2 VOT was different from L1 VOT. Five L1 English Americans and 5 L1 Japanese read the same lists of English and Japanese words containing word initial /p/, /t/, and /k/. In all, 880 tokens
(10 talkers $\times 2$ languages $\times 11$ words $\times 4$ tokens) were examined. For both groups and individuals, based on measures of difference and comparisons with age-matched monolinguals (5 American and 13 Japanese), the findings were (a) slight evidence of L1 VOT change, but (b) robust evidence of new L2 categories being formed. By far the most common bilingual pattern observed was L2 compromise VOT, in which the L1 VOT remained close to monolingual VOT and the L2 VOT was positioned about halfway between that of two age-matched monolingual comparison groups.

3aSC16. Phonetic disambiguation by Korean non-native speakers of English. Jiwon Hwang and Giorgos Tserdanelis (Dept. of Linguist., Stony Brook Univ., NY 11794, gtserdanelis@ sunysb.edu)

This study examined the effect of pragmatic contrast and auditory priming on the production of contrasts such as "mob mop" and "pat pet" by 22 Korean non-native speakers of English. Korean speakers played a card arrangement game with a native speaker of English (a confederate). In the pragmatic condition, critical items were arranged to enable the comparison of the same word (e.g. "mob") in two contexts, one where the situation might force subjects to make the contrast of mob and mop and one where the situation facilitates the confederates recognition of mob because there is no contrasting form. An additional auditory priming condition was induced by the confederate saying a phonetically similar word such as "what is below hob?" before the target "mob." Koreans produced more English-like forms when they had to be distinguished from the contrastive item or when they had been auditorily primed. For both $b / p$ and ae/E contrasts, vowel duration was used to signal the distinction in both conditions. Additionally, for the b/p contrast, closure voicing percentage was significantly longer but only in the pragmatic condition. Overall both conditions have similar disambiguating effect, but have different specific consequences for cueing consonant versus vowel contrasts.

3aSC17. Phonetic similarities between English and Korean sibilants: Implications for second language acquisition. Sang Yee Cheon (Dept. of East Asian Lang. and Lit., Univ. of Hawaii at Manoa, 1890 East West Rd., \#352, Honolulu, HI 96822)

Recent models of L2 acquisition suggest that foreign accent is determined by the degree of articulatory, acoustic, and perceptual similarity between L1 and L2 sounds. This study examined cross-language perceptual and acoustic similarities between Korean and English voiceless sibilant fricatives: Korean lax aspirated /s/ and tense unaspirated /s*/ versus English alveolar /s/ and palato-alveolar / $/ \mathrm{f}$. AX discrimination tests revealed that listeners perceived the non-native contrast in terms of their L1 sound system. To determine acoustic similarity among the fricatives, two acoustic parameters were measured: duration and spectral peak frequency. It was expected that the more acoustically similar two sounds were, the more they would be perceived as the same sound by listeners. However, disparate results were obtained for perceived similarity and acoustic similarity. For instance, Korean lax /s/ and English /J/ before /i/ are acoustically similar, but listeners were easily able to discriminate between them. Perceptual similarity predicts ease of acquisition better than acoustic similarity because a listener's perceptual system incorporates how the sounds are organized in their L1 phonological system. Language teachers need to consider not just degree of similarity between pairs of sounds, but also the differences between the L1 and L2 sound systems as a whole.

3aSC18. Cross-language speech sounds: Differential sensitivity and L2 accent. Miles Munro (Dept. of Cognit. Sci., Univ. of California, Irvine, 3151 Social Sci. Plaza A, Irvine, CA 92697-5100, mmunro@uci.edu)

Individuals vary in their ability to perceive phonetic differences between speech sounds. Individuals also vary in their aptitude for acquiring accurate pronunciation of a second language. The research to be presented 
investigates a probable link between the two with the aim that capacity for phonetic perception may constitute a measure of second language aptitude. In a series of tasks manipulating memory and attention load, Native Mandarin speakers for whom English is a second language were tested on their ability to discriminate non-native speech contrasts that cluster around the Mandarin and English phonetic spaces with varying "distances." Each contrast pair is phonemically meaningful in a significant portion of the world's languages (UPSID 451). These scores were then related to measures of degree of accent in English. Related results examining working memory span will also be reported. Accordingly, an explanation of variability in later-learner L2 accent strength is explained as a consequence of idiosyncratic ability to perceive phonetic differences between speech sounds. This cognitive correlate's account is consistent with the notion that a greater ability to perceive small differences between novel phones is crucial to their accurate acquisition and reproduction.

3aSC19. Performance of auditory search with Japanese speech by native speakers and its learners as L2. Noriaki Asemi (Grad. School of Sci. Technol., Kobe Univ., Rokkodai 1-1, Nada-ku, Kobe, 657-8501 Japan, asemi@kobe-u.ac.jp), Ryoko Hayashi (Kobe Univ., Kobe, Japan), and Kazuhiko Kakehi (Chukyo Univ., Toyota, Japan)

Auditory search experiments using normal Japanese speech (NJS) and time-reversed speech (TRS) [Asemi et al., Acoust. Sci. Technol., 24, 145147 (2003)] demonstrated fast and accurate judgment of the presence or absence of the target NJS among distracting TRSs. For application of an auditory search to measurement of the ability of auditory comprehension of foreign language, the effects of the learning proficiency of Japanese on the speed and accuracy in the detection of the target NJS were examined through auditory search experiments for native and non-native speakers of Japanese. The experiment comprised two parts: an NJS was the target and TRSs were distractors and vice versa. Subjects were requested to judge the presence or absence of a target speech correctly and as quickly as possible when a target and several distracters were presented simultaneously and diotically. Results showed remarkable differences between the performance of natives and non-native speakers in error rates rather than in the reaction times. Moreover, significant correlations were found between the error rates and the learning experiences only when fewer than three utterances were presented simultaneously. [Work supported by MEXT.]

3aSC20. Neurophysiological basis of temporally cued phonetic contrasts in Japanese and American English listeners. Miwako Hisagi, Valerie Shafer (CUNY-Grad. Ctr., Dept. of Speech and Hearing Sci., 365 Fifth Ave., New York, NY 10016, mhisagi@hotmail.com), and Elyse Sussman (Albert Einstein College of Medicine of Yeshiva Univ., New York, NY)

The present study examined American English (AE) listeners' perception of Japanese (JP) temporally cued contrasts of vowels and consonants. The mismatch negativity (MMN) component of event-related potentials (ERPs) was used as a measure to compare passive (visual-attention condition) and active (auditory-attention condition) discrimination in an oddball paradigm. JP nonsense temporally cued vowel ([tado] vs [taado]) and consonant contrasts ([mifi] vs [mifJi]) were presented to JP listeners, for whom the temporal cues are contrastive, and to $\mathrm{AE}$ listeners who have no knowledge of JP. The following results were obtained. (1) With respect to the type of contrast, MMNs were larger for vowel contrasts than consonant contrasts. (2) In the auditory-attention condition both groups showed a robust MMN to vowels. (3) In the visual-attention condition JP also showed a robust MMN, while AE showed a smaller amplitude or absent MMN. This group difference was significant for the vowel contrast. (4) Interestingly, in the auditory-attention condition, $\mathrm{AE}$ showed a larger late negativity at frontal sites for vowel contrasts than JP. The results support the hypothesis that first-language perception is an overlearned process that may interfere with second-language speech perception. [Work supported by NSF.]

3aSC21. Vowel perception in second language acquisition. Mariche Garcia Bayonas (Dept. of Romance Lang., at Greensboro, Univ. of North Carolina, 2314 HHRA Bldg., UNCG, P.O. Box 26170, Greensboro, NC 27402-6170)

This study investigates the perception of ten English and five Spanish vowels by native-speakers (NS) and learners (L) at two levels of acquisition, and compares these vowels cross-linguistically. Research on the acquisition of vowels indicates that learners can improve their perception with exposure to the second language (L2) [Bohn and Flege (1990)]. Using Flege's (1995) Speech Learning Model as a theoretical framework, this investigation incorporated a method of adjustment (MOA) task [Johnson et al., (1993)] used to analyze NS perception and production data, to add the dimension of the L2 learner data. The research question was: Are vowels selected differently by NS and L in an MOA task depending on level of proficiency? Spanish learners $(n=40)$ and English learners $(n=80)$ completed MOA tasks in which they were exposed to 120 synthetically produced vowels to analyze spectral variation in the acquisition of both sound systems, and how the learners vowel system may vary from that of the NS. In the MOA tasks they were asked to select which synthesized vowel sounds most resembled the ones whose spelling was presented to them. Results indicated that vowels are selected differently by NS and L, but there are differences among vowels.

3aSC22. Multidimensional category-goodness-difference assimilation of Canadian English /i/ and /I/ to Spanish /i/. Geoffrey Stewart Morrison (Dept. of Linguist., Univ. of AB, Edmonton, AB, T6G 2E7, Canada,gsm2@ualberta.ca)

Morrison [J. Acoust. Soc. Am., 117, 2401 (2005)] theorized that L1Spanish learners of English initially identify the Canadian English /i/-/I/ contrast via a multidimensional category-goodness-difference assimilation to Spanish /i/, with more-Spanish-/i/-like vowels (shorter vowels, lower F1 and higher F2) labelled as English /I/ and less-Spanish-/i/-like vowels (longer vowels, higher F1 and lower F2) labelled as English /i/. The L1Spanish listeners' use of duration is positively correlated with L1-English speakers' productions, but their use of spectral cues is negatively correlated; hence, exposure to English results in increased use of duration cues and reduced use of spectral cues, leading to unidimensional duration-based perception. Since, in L1-English speakers' productions, spectral properties are partially correlated with duration properties, L2-English learners can use duration-based perception as a bootstrap for learning L1-English-like spectral-based perception. Results from a new study are presented. Unlike the previous study, synthetic stimuli in the new study included vowel inherent spectral change (VISC). Results were consistent with the theory above: Vowels that were poor matches for Spanish /i/ because they had converging-VISC were labelled as English /i/, and vowels with no VISC were labelled as English /I/. [Work supported by SSHRC.]

3aSC23. Multiple cue interpretation in non-native phoneme perception: Korean listeners' perception of English /s/ in varied prosodic contexts. Miran Kim (Dept. of Linguist., SUNY at Stony Brook, Add., S201, SBS Bldg, Stony Brook, NY 11794-4376, mrkim@ic.sunysb.edu)

This paper investigates prosodic influences on the non-native perception of English /s/ by Korean listeners, whose phonemic system includes a two-way distinction of fricative, plain /s/ vs. fortis $/ \mathrm{s} * /$. English stimuli varied in lexical stress and prosodic domain appear to be relevant to the Korean listeners perceptual responses such that more /s*/ responses to stimuli are obtained with more strengthening effects such as lexical stressed and phrase boundary initial position. Analyses of the relation 
between the spectral properties of the stimuli and perception shows that prosodic effects on /sV(C)/ syllables are compatible with the characteristics that have been reported as critical attributes in the distinction of plain and fortis fricatives in Korean. Spectral measurements include F0, H1-H2 $(\mathrm{dB})$, and H1-F2 (dB) values of postfricative vowels, together with the spectral characteristics of frication noise. The relation between prosodic strength in the stimuli and Koreans /s-s*/ perceptual responses implies that Korean listeners interpret prosodic information associated with English /s/ as a part of the segmental properties of English /s/ instead of attributing them to prosodic effects. This leads us to conclude that phonetic information is not limited to a segment-to-segment or prosody-toprosody transmission, but it can be overlapped in multiple phonetic dimensions.

3aSC24. Identification of English consonants by Korean students. Byunggon Yang (English Education Dept., Pusan Natl. Univ., 30 Changjundong, Keumjunggu, Pusan 609-735, South Korea)

Previous studies have shown that Korean students have difficulty identifying some English consonants that are not in the Korean sound inventory. The aim of this study was to examine the accuracy score of English consonants correctly identified by 130 college students in order to find out English consonants with listening problems for the Korean students. The subject's task was to identify one of the minimal pairs played in a quiet laboratory classroom. One hundred minimal pairs consisted of syllables with various onsets or codas: stops, fricatives, affricates, liquids, and nasals. Results were as follows: First, types of the correctly identified English consonants varied according to the level of each student's total score. Second, the lower the group level, the lower the accuracy score for each consonant group divided by its manner. Third, those consonants in coda position of the test words were more difficult for the students to identify than those in onset position. Fourth, the subjects showed lower accuracy in the perception of any contrast with a fricative onset or coda. Finally, the students still had problems telling voiced from voiceless consonants, especially in coda position. Future studies will focus on examining students' production data to trace their listening problems.

3aSC25. Identification of English / $r /$ and $/ / /$ in noise: The effects of baseline performance. Kazuo Ueda (Dept. Appl. Inf. and Commun. Sci., Kyushu Univ., 4-9-1 Shiobaru, Minamiku, Fukuoka 815-8540, Japan, ueda@design.kyushu-u.ac.jp), Ryo Komaki, and Takahiro Adachi (ATR, Soraku-gun, Kyoto 619-0288, Japan)

The effects of baseline performance on identification of English /r/ and $/ 1 /$ in noise by Japanese listeners were examined. Japanese and American listeners' perception of /r/ and /1/ was measured under various signal-tonoise ratios. Each Japanese listener's baseline performance was also observed with an identification test with a large set of stimuli. Generally, the signal-to-noise ratio had a similar effect across Japanese listeners of different baseline performances, although the overall accuracy was dominated by the baseline. When 15 days of /r/-/1/ identification training in quiet were applied to a group of Japanese listeners, the training effect generalized to identification performance in noise. However, the performance of Japanese listeners did not reach the level that the native listeners exhibited. [Work supported by JSPS and 21st Century COE, Kyushu University.]

3aSC26. Effects of learners' L2 proficiency and acoustic/semantic contexts of stimuli on the phoneme identification training. Yuko Ikuma and Reiko Akahane-Yamada (ATR, 2-2-2, Hikaridai, Seika-cho, Soraku-gun, Kyoto, 619-0288, Japan)

Former studies investigated that word recognition by L2 learners is affected by both acoustic and semantic contexts [Rothwell and AkahaneYamada, J. Acout. Soc. Am. 112, 2386 (2002)]. It was also shown that these two context effects lead to different achievement in perception train- ing [Ikuma and Akahane-Yamada, J. Acout. Soc. Am. 115, 2393 (2004)]. In this study, native speakers of Japanese were trained to identify English $/ \mathrm{r} /$ and /1/ within words or sentences. L2 proficiency level for each trainee was also measured by a short written test regarding vocabulary and grammar. Results showed that training with semantically contextual stimuli improved the identification accuracy for stimuli with semantically contextual cues, but not for stimuli without semantic cues. Furthermore, the L2 proficiency level correlated only with the improvement in identification accuracy of target phonemes embedded in the semantically contextual sentences. These results suggest that L2 learners tend to use semantic cues rather than difficult acoustic cues. However, semantic context may moderate the effect of auditory perception training. Thus, training with semantically neutral stimulus is necessary to improve perception ability even for high proficiency level learners. [Work supported by JSPS.]

3aSC27. Asymmetry in discrimination of an American English contrast, /l-r/, by adult Japanese learners of English. Teruaki Tsushima (Ryutsu Kagaku Univ., 3-1, Gakuen-Nishi-Machi, Nishi-Ku, Kobe, 651-2188, Japan)

The present study provided further evidence on an asymmetry in discrimination of an American English contrast, /1-r/, by Japanese listeners. Experiment $1(N=14)$ examined stimulus order effects in discrimination of the contrast in the AX discrimination procedure with an ISI of 300 and $1500 \mathrm{~ms}$, using naturally produced stimuli. The results showed discrimination accuracy was significantly lower when /l/ was followed by /r/ than otherwise, under the ISI of $1500 \mathrm{~ms}$. Experiment $2(N=17)$ examined whether discrimination significantly differed when a typical /1/ was paired with an ambiguous $/ \mathrm{r} /$ and vice versa, using synthesized stimuli from a ten-step continuum (i.e., stimulus 1-7 and 4-10). The participants were tested on their identification of the stimuli, and discrimination of the stimulus pairs with the ISI of 300 and $1500 \mathrm{~ms}$. The discrimination sensitivity (i.e., $d^{\prime}$ ), based on the identification performance, was not significantly different between the two pairs. In the discrimination test, however, the sensitivity was significantly lower in the pair including the typical /l/ than in the other, under the ISI of $1500 \mathrm{~ms}$. The results support the hypothesis that categorization of a relatively nativelike speech stimulus (i.e., /1/) in a discrimination task may induce some perceptual assimilation process.

3aSC28. Context really matters!- The influence of input types on L2 speakers' perception of English liquids. Sally Chen and Janice Fon (Grad. Inst. of Linguist., Natl. Taiwan Univ., 1 Roosevelt Rd., Sec. 4, Taipei 106, Taiwan)

This study aims to investigate how different input types can help improve the perception of language learners on L2 sounds. Twenty-four college students of a remedial English class are divided into three groups and are given audio inputs of different types. The first group listens to a wordlist, the second group to a story incorporating all the words on the wordlist, and the third group to a piece of classical music, serving as the control. The wordlist consists of 56 monosyllabic English words with word-initial liquids or liquid-containing clusters. Before the listening task, all three groups are given an online $1 / \mathrm{r}$ perception test containing all the words on the wordlist (pretest). After the listening task, the same perception test is again administered, including the original 56 words and another 56 phonotactically matched pseudo- $\mathrm{V}$ words (posttest). Both accuracy and reaction time are measured for the two perception tests. Preliminary results showed that all participants had significantly higher accuracy and shorter RT for real words in the posttest. The story group showed the biggest improvement for both measures, followed by the wordlist group. The control showed the least improvement. 
3aSC29. Categorization of a sibilant+ vowel continuum in Japanese, Mandarin, and English. Terrance M. Nearey, Aya Okamoto, Chunling Zhang, and Ron I. Thomson (Dept. of Linguist., Univ. of AB, Edmonton, $\mathrm{AB}, \mathrm{T} 6 \mathrm{G}$ 2E7, Canada)

A continuum of 168 synthetic sibilant + vowel stimuli was categorized by listeners of three languages (Japanese, Mandarin, and English). The contoid portion of each stimulus consisted of a 190-ms high-pass filtered white noise whose cutoff frequency varied from 1800 to $3800 \mathrm{~Hz}$ in 7 steps. (Signals were low-pass filtered at $8500 \mathrm{~Hz}$ before resampling to $22.05 \mathrm{kHz}$ for playback.) Each (cascade formant) vocoid portion was 210 $\mathrm{ms}$ in duration varying in three $F 1$ levels (from 300 to $430 \mathrm{~Hz}$ ) crossed with four $F 2$ levels (from 850 to $2100 \mathrm{~Hz}$, with fixed $F 3$ through $F 5$ ). Each of the 84 noise + vocoid patterns was combined with two different F2 transition patterns, designed to slightly favor more alveolar or more palatal fricative responses. Appropriate response sets were determined in pilot studies for each language by native speakers on the research team. Corresponding software was designed to allow computer-controlled categorization. Data collection for 9 Japanese, 20 Mandarin, and 4 English listeners is complete (more is planned). Responses to the stimuli spanned at two or three sibilant and several (high-to-mid) vowel categories in each language. Possible effects of language-specific phonotactics on the response patterns will be discussed.

3aSC30. Perceptual cue learning. Grant McGuire (The Ohio State Univ., 222 Oxley Hall, 1712 Neil Ave., Columbus, OH 43210, mcguire@ling.osu.edu)

This paper describes results from an on-going perceptual training study in which native listeners of English are trained perceive a categorical difference between Polish alveopalatal and retroflex sibilants. The stimuli consist of naturally produced, modified two-dimensional stimuli varying by fricative noise and formant transition cues. Subjects are trained to categorize using only the fricative dimension, formant transition dimension, or both dimensions. Testing consists of a two-alternative forcedchoice discrimination paradigm and stimulus labeling. Initial results from pilot work and subjects in the fricative dimension training group demonstrate that some listeners can easily attend to either dimension independently for categorization where training focuses attention and sharpens boundaries while others rely heavily on the vocalic cues, only attending reliably to frication noise cues with several training sessions or through verbal instruction. A handful of subjects did not show any improvement due to training; most of these subjects also showed no initial categorization strategy. Moreover, sensitivity to the fricative dimension and boundary increases for all learners (measured in $d^{\prime}$ ), with no corresponding increase or decrease in sensitivity to vocalic cues. Further results from other training conditions will also be reported. [Work supported by NIHNIDCD.]

3aSC31. Cantonese and Japanese listeners' processing of Russian onset and coda stops. Alexei Kochetov (Dept. of Linguist., Simon Fraser Univ., 8888 Univ. Dr., Burnaby V5A 1S6, Canada, alexei_kochetov@sfu.ca), Connie K. So (Univ. of Western Sydney, Australia), and Nicole Carter (Simon Fraser Univ., Burnaby V5A 1S6, Canada)

The study investigated influences of native phonological and phonetic knowledge on Cantonese and Japanese listeners' processing of non-native stop consonants. Phonologically, Cantonese Chinese has a three-way place contrast in stops in onset and coda positions; Japanese has a similar contrast, however, only in onset position. Phonetically, Cantonese coda stops are acoustically unreleased; Japanese stops followed by devoiced vowels are acoustically similar to released coda stops in other languages, such as Russian. Two groups of listeners, native speakers of Hong-Kong Cantonese and Japanese, were presented with sequences of Russian voiceless stops $(\mathrm{VC} 1 \# \mathrm{C} 2 \mathrm{~V}$, where $\mathrm{C} 1 / \mathrm{C} 2=/ \mathrm{p} /, / \mathrm{t} /$, or $/ \mathrm{k} /)$. Two tasks were employed: (i) identification of onset or coda stops and (ii) discrimination of sequences that differed in either onset or coda consonant. Both groups performed equally well in the identification and discrimination of onset stops. However, Japanese listeners performed significantly better than Cantonese listeners in the identification and discrimination of coda stops. The findings suggest that in non-native listening, the low-level native phonetic knowledge-the acoustic realization of stop place contrasts-can override the higher-level phonological knowledge-syllable structure constraints on the distribution of place features. [Work supported by Social Sciences and Humanities Research Council of Canada.]

3aSC32. Relationships between the intelligibility and acoustic properties of native English and Mandarin-accented English. Rachel Hayes-Harb (Dept. of Linguist., Univ. of Utah, 255 S. Central Campus Dr., Rm. 2330, Salt Lake City, UT 84112), Bruce Smith (Univ. of Utah, Salt Lake City, UT 84112), Tessa Bent (Indiana Univ., Bloomington, IN 47405), and Ann Bradlow (Northwestern Univ., Evanston, IL 60208)

Studies examining effects of talkers' and listeners' linguistic backgrounds on speech intelligibility have produced mixed results, and relatively little is known about which acoustic-phonetic features of native and non-native speech are responsible for many findings. These issues are addressed here via investigation of how acoustic properties of final consonant voicing contrasts influence word intelligibility. Native English and native Mandarin talkers' productions of English words were presented in isolation to native English and native Mandarin listeners in an identification task. Intelligibility findings were related to preceding vowel duration, stop consonant duration, burst duration, and proportion of voicing during stop closure. As expected, native English listeners were more accurate identifying words spoken by native than non-native talkers. In contrast to previous findings of an interlanguage speech intelligibility benefit (ISIB) for sentences, non-native listeners found native speech more intelligible than non-native speech. However, non-native listeners found non-native speech more intelligible than did native listeners. For native talkers, all four acoustic measures contributed significantly to correct identification of voicing contrasts by both native and non-native listeners (with closure voicing most salient). In contrast, for non-native talkers, listeners tended to rely primarily on closure voicing and burst duration. Ramifications for ISIB will be discussed.

3aSC33. English listeners perceptual assimilations for Zulu sounds: Evidence in support of the articulatory organ hypothesis. Andrea Levitt (Wellesley College, 106 Central St., Wellesley, MA 02481, and Haskins Labs., 300 George St., New Haven, CT 06511, alevitt@wellesley.edu), Catherine Best (Univ. Western Sydney, Penrith South NSW 1797, Australia), Louis Goldstein (Yale Univ., New Haven, CT), and Angela Carpenter (Wellesley College, Wellesley, MA 02481)

The articulatory organ hypothesis predicts that listeners' favored perceptual assimilations for non-native sounds will be native sounds that are produced by the same articulatory organ [L. Goldstein and C. Fowler in Phonetics and Phonology in Language Comprehension and Production, 159-207 (2003)]. Using discrimination paradigms, we tested Englishspeaking, adult listeners' perceptual assimilations for four non-native Zulu consonants to see whether assimilations are stronger for sounds articulated with the same vocal-tract organ or those with the same constriction degree. For each Zulu sound, three possible English-language assimilations were provided: a best match, based on the most frequent consonant assimilation provided by the subjects in a previous study [C.T. Best, G.W. McRoberts, and E. Goodell, J. Acoust. Soc. Am. 109, 775-794 (2001)], an assimilation match where the consonant uses the same articulatory organ, and one where the consonant uses the same constriction degree as the Zulu target. For example, for the bilabial implosive, the best match was /b/ (same articulator and constriction degree), the same organ match $/ \mathrm{w} /$, and the same constriction match /d/. Although there were differences in the patterns of responses to the four sounds, overall the results provided support for the articulatory organ hypothesis predictions. 
3aSC34. Learning of non-native tonal contrasts with or without tonal context. Valter Ciocca (Div. of Speech \& Hearing Sci., Univ. of Hong Kong, 34 Hospital Rd., Hong Kong, vciocca@ hkusua.hku.hk), Alexander L. Francis, and Yanhong Zhang (Purdue Univ., West Lafayette, IN 47907-2038)

The learning of non-native (Cantonese) tonal categories by native speakers of another tonal language (Mandarin) was investigated under two learning conditions. For listeners in the tonal context (TC) group, learning occurred by listening to target tones within various carrier phrases. Listeners in the no-context group ( $\mathrm{NC}$ ) were trained using target tones produced as single words. Training stimuli were produced by the same speakers for both groups. A high-variability procedure with feedback was used for training. Sixteen listeners (eight in each group) took part in eight sessions over a period of about 1 month. Categorical perception of Cantonese tones was measured before training (following a brief familiarization session) and after training. For both groups, tone perception was tested for one level- and one contour-tone continua using identification and discrimination procedures. Identification performance before and after training did not differ between the two groups for the level-tone continuum, showing evidence of learning of the (non-native) mid-level category. For the contour-tone continuum, the TC group showed better learning of the (non-native) low-rising/low-level contrast, as well as better perceptual distinction between low-rising and high-rising tones. [Work supported by Hong Kong RGC Grant HKU 7224/03H.]

3aSC35. Native and non-native identification of acoustically modified Mandarin tones. Chao-Yang Lee (School of Hearing, Speech and Lang. Sci., Ohio Univ., Athens, OH 45701), Liang Tao, and Zinny S. Bond (Ohio Univ., Athens, OH 45701)

Lexical tones have been known to be one of the most difficult aspects of tone languages to master for non-native learners. Extending upon Gottfried and Suiter's study [J. Phonetics 25, 207-231 (1997)] of tone identification in silent center syllables, four experiments examined how well Mandarin tones can be identified with partial acoustic information and whether native and non-native listeners show differences in the use of the limited acoustic information. Twelve minimal tone pairs including all six tonal contrasts in Mandarin were digitally processed to generate four types of stimuli: silent-center, center-only, onset-only, and intact syllables. The stimuli were presented in the original carrier phrase, excised from the carrier phrase, or excised and pasted onto another carrier phrase. Participants included 40 non-native speakers with 1-3 years of Mandarin instruction at Ohio University and 40 native speakers. Both learners and native speakers could identify the tones under all modification conditions at better than chance levels. For both groups, the onset-only syllables were the most difficult. Tone 2 received the fewest correct identifications and longest response times, being confused with tone 3 .

3aSC36. Acoustic correlates of English lexical stress produced by native speakers of Mandarin Chinese. Yanhong Zhang and Alexander L. Francis (Program in Linguist., Purdue Univ., West Lafayette, IN 47907)

Native speakers of Mandarin Chinese have difficulty producing nativelike English stress contrasts. Acoustically, English lexical stress is multidimensional, involving manipulation of fundamental frequency (f0), duration, amplitude, and vowel quality. Errors in any or all of these correlates could result in poor realization of the stress contrast, but it is unknown which cues are most problematic for Mandarin speakers. Here we compared the use of these correlates in the production of lexical stress contrasts by 20 Mandarin and English speakers. Results showed that Mandarin speakers produced significantly less acceptable stress contrasts, although they did use all four acoustic correlates to distinguish stressed from unstressed syllables. Mandarin and English speakers' use of amplitude and duration were comparable for both stressed and unstressed syllables, but Mandarin speakers signaled stressed syllables with a higher f0 than English speakers. There were also significant differences in formant patterns across groups, suggesting that Mandarin speakers were able to achieve appropriate vowel reduction in certain unstressed syllables, but not in others. Results suggest that Mandarin listeners' production of lexical stress contrasts in English is influenced in part by native-language experience with Mandarin lexical tones, and in part by similarities and differences between Mandarin and English vowel inventories.

3aSC37. On the characteristics of English speech timing control by Japanese learners. Hajime Tsubaki, Shizuka Nakamura, and Yoshinori Sagisaka (GITI Waseda Univ., 29-7 Bldg. 1-3-10, Nishi-Waseda, Shinjuku-ku, Tokyo 169-0051, Japan, hjm-tsubaki@asagi.waseda.jp)

This paper introduces analysis results of temporal characteristics of English language uttered by Japanese subjects aiming at automatic evaluation of L2 prosody control. As a preliminary study, 11 sentences uttered by about 200 Japanese children were compared with native's speech. The correlation between subjective scores on temporal naturalness and differences in segmental durations were measured in different speech units at every position for sentence by sentence. A wide range of correlation differences were observed such as from -0.70 to 0.23 at unstressed syllables, from -0.33 to 0.19 at stressed syllables, and from -0.70 to -0.04 at function words. The strongest negative correlation -0.70 at unstressed syllables and at function words reflects L1 characteristics of mora-timing characteristics of Japanese. Further analysis results will be given at the conference with additional huge L2 speech database currently under construction. [Work supported in part by Waseda Univ. RISE research project of "Analysis and modeling of human mechanism in speech and language processing" and Grant-in-Aid for and Scientific Research A-2, No. 16200016 of JSPS.]

3aSC38. Acquisition of Japanese pitch-accent by non-native speakers. Tomoko Kozasa (Dept. of Linguist., California State Univ., Fresno, 5245 N Backer Ave., M/S PB92, Fresno, CA 93740-8001)

Vowel duration and $F 0$ movement in Japanese pitch-accented words produced by non-native speakers were investigated. Phonemic vowel length and pitch accent are two prosodic properties of Japanese that are problematic for language learners. The vowel length distinction is typically realized by durational differences, and pitch accent involves $F 0$ movement from high to low within an accented word. Equal numbers of target words and filler words were produced by 50 speakers by repeating after audio recorded Japanese sentences one at a time. The stimulus sentences were uttered by a native speaker of Tokyo Japanese. Speakers were randomly divided into two groups. For group A, a visual stimulus indicating the native speaker's pitch contour for the target word appeared right after each audio stimulus. Speakers were instructed to follow the visual stimulus as they produced the target word. No visual stimulus was provided to group B. A sufficient durational distinction between long and short vowels was produced by both groups. However, group B failed to produce adequate amount or direction of $F 0$ movement. These results suggest that explicitly showing pitch contour to language learners improves their pronunciation of pitch accent.

3aSC39. Native speakers and Korean-speaking advanced learners of English show similar discrimination of English prosodic contours. Hyekyung Hwang and Amy J. Schafer (Dept. of Linguist., Univ. of Hawaii, 569 Moore Hall, 1890 East-West Rd., Honolulu, HI 96822, hyekyung@hawaii.edu)

Previous studies of native versus non-native perceivers argue that phonology can shape perception (e.g., Huang, 2001; Hume et al., 1999). The phonological inventory of prosodic phrasing contours differs between Korean and English. Phonologically, Korean accentual phrases have final rises (within intonation phrases), although this varies at the phonetic level, while English intermediate phrases (ips) can end in falling, rising, or level contours. Previous work has suggested that advanced learners (L2ers) use 
ips markedly less effectively than native speakers (L1ers) to recover English syntactic structure (Hwang and Schafer, 2005). This study investigated whether that is due to perceptual differences. L1ers and L2ers listened to pairs of phrases in an AX task that crossed boundary strength (same versus different size) with contour: rising $\left(\mathrm{L}^{*} \mathrm{~L}^{*} \mathrm{H}-\right)$ versus level ( $\mathrm{L}^{*} \mathrm{~L}^{*} \mathrm{~L}-$ or $\left.\mathrm{L}^{*} \mathrm{~L}^{*}\right)$ or falling $\left(\mathrm{H}^{*} \mathrm{H}^{*} \mathrm{~L}-\right)$ versus level $\left(\mathrm{H}^{*} \mathrm{H}^{*} \mathrm{H}\right.$ - or $\left.\mathrm{H}^{*} \mathrm{H}^{*}\right)$. Little variation was found between L1ers' and L2ers' discrimination, and L2ers' discrimination patterns correlated highly with L1ers' ( $r$ $=0.966$ ). For both groups a falling-level contrast was more salient than a rising-level contrast (in the context tested) and boundary strength contrasts were more difficult than contour contrasts. The results suggest that the L2ers' poor use of ips in comprehension is likely due to difficulty with prosody-syntax mappings.

3aSC40. The effect of phonological knowledge on word learning in second language acquisition. Junko Maekawa (Dept. of Speech-Lang.-Hearing, Univ. of Kansas, 1000 Sunnyside Ave., 3001 Dole Ctr., Lawrence, KS 66045-7555, junko@ku.edu)

Past research in first language (L1) acquisition suggests that phonological knowledge (individual sounds and sound sequences) influences word learning. In second language (L2) word learning, phonological knowledge is dependent on the mapping of L2 sounds to L1. This study explores how the L2-L1 phonological mapping affects L2 word learning for native speakers of Japanese learning English. The learners phonological knowledge was explored through a perceptual discrimination task (same-different task) and a production probe containing English real words and nonwords. L2 word learning was explored through exposure to novel words and comprehension and production testing. The results of the same-different task showed that $\mathrm{r}-1$ and $\theta$-s contrasts were the most difficult to perceive, supporting findings from past research. For the production probe, $\mathrm{w}, \mathrm{t}$, and $\mathrm{s}$ were produced more accurately than $\mathrm{r}, 1$, and $\theta$ in both real words and nonwords. For L2 word learning, nonwords with w, t, and $\mathrm{s}$ were learned more rapidly than nonwords with $\mathrm{r}, 1$, and $\theta$ in production, but not comprehension. Taken together, phonological knowledge appears to constrain L2 word learning for language expression, but not comprehension. This mirrors the findings for L1 acquisition.
3aSC41. Context effects in recognition of English disyllabic word and nonwords by native and non-native listeners. Robert Felty (Dept. of Linguist. and German, Univ. of Michigan, 3110 Modern Lang. Bldg., 812 E. Washington St., Ann Arbor, MI 48109-1275) and José Benkí (Univ. of Michigan, Ann Arbor, MI 48106)

30 native $(\mathrm{L} 1=$ English $)$ and 30 non-native $(\mathrm{L} 1=$ German $)$ speakers listened to English CVCCVC words and nonwords mixed with noise. Of the words, half were monomorphemic and half bimorphemic. The $j$-factor model [Boothroyd and Nittrouer, J. Acoust. Soc. Am. 84, 101-114 (1988)] was used as a measure of lexical context effects. For both native and non-native speakers, words showed greater context effects than nonwords, though the difference was not as large for non-native speakers. Monomorphemic words also exhibited greater context effects than bimorphemic words, though only for native speakers. In an items analysis, a negative correlation with $j$ was found for lexical frequency and a positive correlation for neighborhood density, for both native and non-native speakers, though more pronounced in native speakers. Misperceptions between native and non-native speakers were also compared, with non-native speakers showing patterns predictable by phonological differences between the two languages. The $j$-factor results extend previous research using $\mathrm{CVC}$ stimuli, providing additional support for its efficacy as a measure of context effects. The differences (and lack there of for non-native speakers) between mono- and bimorphemic words are interpreted as evidence in support of a combinatorial model of lexical access. [Work supported by NIH/NIDCD.]

3aSC42. Recognizing English words by Japanese speakers. Kiyoko Yoneyama (Dept. of English, Daito Bunka Univ., 1-9-1 Takashimadaira, Itabashi, Tokyo, 175-8571, Japan, yoneyama@ic.daito.ac.jp)

Japanese listeners are influence by word frequency and neighborhood density (Yoneyama, 2002) as in other languages such as English. Interestingly, non-native listeners are also affected by word frequency and neighborhood density when they recognize English (e.g., Bradlow and Pisoni, 1999; Imai et al., 2005). Based on the previous studies of neighborhood density by non-native listeners, Yoneyama (2006) investigated whether Japanese listeners learning English employ two types of lexical information (word frequency and neighborhood density) when they recognize English words, as in Imai et al. (2005). The results were different from the ones of Imai et al. (2005). Japanese listeners were sensitive to both probabilistic phonotactics and word frequency. A strong correlation between probabilistic phonotactics and neighborhood density still predict Japanese listeners can be influenced by neighborhood density in English word recognition. A critical difference between Yoneyama (2006) and Imai et al. (2005) is that they did not use the exact same materials, although Yoneyama (2005) selected the words as described in a conference paper of an earlier version of Imai et al. (2005). This study reports the results of the experiment in which the materials of Imai et al. (2005) were used. 


\title{
Session 3aSP
}

\section{Signal Processing in Acoustics: Sensor Array and Its Applications}

\author{
Henry Cox, Cochair \\ 4350 N. Fairfax Dr., Ste. 470, Arlington, VA 22203 \\ Masato Miyoshi, Cochair \\ NTT Communication Science Labs., 2-4 Hikaridai, Seika-cho, Soraku-gun, Kyoto 619-0237, Japan
}

Chair's Introduction-8:00

Invited Papers

8:05

3aSP1. Application of a microphone array to the automatic structuring of meeting recordings. Futoshi Asano, Jun Ogata, and Yosuke Matsusaka (AIST, Central 2 1-1-1 Umezono, Tsukuba, Ibaraki 305-8568, Japan)

A microphone array system applied to the automatic structuring of meeting recordings is introduced. First, speakers in a meeting are identified at every time block $(0.25 \mathrm{~s})$ by sound localization using a microphone array, and then the speech events are extracted (structuring). Next, overlaps and insertions of speech events such as "Uh-huh," which greatly reduce the performance of the automatic speech recognition, are separated out. The difficulty in eliminating overlaps and insertions is that the overlapping sections are sometimes very short, and data sufficiently long for conventional separation techniques, such as independent component analysis, cannot be obtained. In this system, a maximum-likelihood beamformer is modified so that all the information necessary for constructing the separation filter, such as speaker location and spatial characteristics of noise, are estimated from the recorded data based he structuring information. Evaluation of the performance of the speech event separation by automatic speech recognition is also presented.

\section{$8: 25$}

3aSP2. Eigenbeam beamforming for microphone arrays. Jens Meyer and Gary W. Elko (mh acoustics LLC, 25-A Summit Ave., Summit, NJ 07901)

In this talk, we describe a beamforming spherical microphone array consisting of many acoustic pressure sensors mounted appropriately on the surface of a rigid sphere. The associated beamformer is implemented in a two-stage process: a first stage that decomposes of the sound-field into spatially orthonormal spherical harmonics, which are called eigenbeams, and a second stage that combines the eigenbeams to form the overall output beampattern. It is shown that this approach allows for a computationally efficient, flexible, and scalable beamformer structure. As an example of beamforming flexibility, an acoustic zoom feature will be described. This application enables continuous variability of the beamwidth in a simple and elegant manner. Simultaneously, the array beampattern can be steered without affecting the beampattern shape. There are many applications for such a feature, one being the coordination of the acoustic and image scenes of microphone and a video camera.

\section{8:45}

3aSP3. Effect of low independency on independent component analysis and countermeasures against it. Hidefumi Kobatake (Tokyo Univ. of Agriculture and Technol., 3-8-1, Harumi-cho, Fuchu 183-8538, Japan, kobatake@cc.tuat.ac.jp) and Xuebin Hu (Fuji Photo Film Co., Ltd., Chuo-ku, Tokyo 104-0061, Japan)

Independent component analysis (ICA) is extensively adopted in blind signal separation (BSS). It works well in general. However, processing must be made at every short-time interval and, as a consequence, the assumption of independency cannot always hold due to insufficient data length. This local low-independency can be the cause of degradation in the overall separation performance. In this paper, two approaches to solve that problem caused by local low-independency between source signals are presented. The first one is the combination of ICA-based BSS with beamformer (BF). The BF does not depend on the independence assumption. In the proposed method, ICA and BF are applied in parallel. The frequency components separated by those methods are evaluated by each other and the frequency bin with higher segmental similarity measure is adopted. The second one is a kind of TDD method with a recursive algorithm. A criterion on the performance of separation is introduced. The proposed method has a function to improve the separation filter characteristics recursively. Experiments to evaluate the performance of those methods and demonstrations are given.

\section{9:05}

3aSP4. Some current challenges in multichannel acoustic signal processing. Walter Kellermann (Univ. Erlangen Nuremberg, Cauerstr. 7, 91058 Erlangen, Germany,wk@LNT.de)

Hands-free human/machine communication involves major challenges for today's state of the art signal processing if the humans should not be forced to carry or wear any technical gear and should be free to move. The spatial domain sampled by multiple loudspeakers for reproduction and multiple microphones for signal acquisition allows for spatialized listening experiences and for 
spatially selective acoustic scene analysis, respectively. Along with the possibility to serve several human communication partners simultaneously, such systems also introduce the need for localization algorithms. Three main types of impairment have to be addressed by signal processing algorithms to fulfill the promises of the general concept: first, acoustic feedback from the loudspeakers into the microphones; second, ambient noise and undesired pointlike interferers; and, finally, echoes and reverberation as picked up by the human ear when listening to the loudspeaker signals or as recorded by the microphones that actually should record only the direct-path component of the source signal. Accordingly, some of the current challenges in multichannel acoustic echo cancellation, in spatially selective noise and interference suppression, in source separation and localization, and in dereverberation are investigated.

\section{9:25}

3aSP5. Sound source tracking for security camera using sensor array utilizing object-related transfer function. Tsuyoshi Usagawa, Toshimichi Takada, and Yoshifumi Chisaki (Grad. School of Sci. and Technol., Kumamoto Univesity, 2-39-1 Kurokami, Kumamoto 860-8555, Japan, tuie@ cs.kumamoto-u.ac.jp)

A frequency-domain binaural model has been proposed as a computer model of human auditory system, and it can estimate the direction of arrival (DOA) of target sound source with only two inputs [Nakashima et al., Acoust. Sci \& Tech. 21, $172-178$ (2003)]. It utilizes a database of interaural level and phase differences obtained from head-related transfer function (HRTF) for DOA estimation. Since the interaural level and phase differences arise from the diffraction of head, similar ones can be obtained from the diffraction of an object, such as a transparent cover of security camera. In this paper, a source tracking method which utilizes an object-related transfer function is proposed. Three sensors attached on a dome-shaped camera cover are used, and the interchannel level and phase differences obtained from object-related transfer function are used for DOA estimation. The performance of sound source tracking is discussed based on the accuracy of DOA estimation as well as observation latency by the computer simulation. [Research partially supported by the MEXT, Grant-in-Aid for Scientific Research (C), 2006, 18500135 and carried out by the Cooperative Research Project Program of the RIEC, Tohoku University (Grant \#H16.A02).]

\section{9:45}

3aSP6. Low-complexity design schemes for broadband electroacoustic arrays. Michael Goodwin (Creative Adv. Technol. Ctr., 1500 Green Hills Rd., Ste. 205, Scotts Valley, CA 95066, mgoodwin@atc.creative.com)

A variety of methods has been described in the literature for realizing broadband linear electroacoustic arrays, i.e., arrays which exhibit a nearly frequency-invariant response. For example, filter-network beamformers can be designed to achieve a specified beam pattern over a wide frequency range. Some applications, such as low-cost consumer audio products, do not involve strict target pattern requirements and call for lower complexity broadbanding solutions. In this presentation, three broadband array design approaches with low processing overhead and implementation cost are described. The key idea is to configure the array such that its response is essentially a magnification of the response of an individual element; the array geometry and beamformer are designed so as to not introduce directionality. In the first method, the array weights are an approximation of the impulse response of an all-pass filter; in the second method, the array elements are nonuniformly spaced; and, in the third method, arbitrary delays are applied to the elemental signals. Relationships to approaches previously described in the literature are discussed, optimization schemes based on minimizing the array response variation are presented, and the effectiveness of the array designs for realizing a robust broadband wide-angle response is demonstrated.

\section{0:05-10:20 Break}

\section{Contributed Papers}

10:20

3aSP7. Three-dimensional microphone array intensity vector sensors for measurement of various acoustical properties in different spatial environment. Khalid H. Miah and Elmer L. Hixson (Univ. of Texas, Austin, TX 78712,miah@mail.utexas.edu)

Precise measurement of different acoustical quantities in different spatial environment has been the key in improving sound-quality and reducing acoustical noise from various sources. In this report, a seven-element 3-D microphone array configuration has been used to measure different acoustical quantities (pressure, particle velocity vectors, intensity vectors, energy density, kinetic and potential energy) in various enclosed environments. Microphones in the array were arranged in an inner and an outer triangular fashion with a reference microphone at the center to address high frequency $(1-6 \mathrm{kHz})$ and low frequency $(100-1000 \mathrm{~Hz})$ components of sound signals. Statistical distribution of acoustic field has been used for measurements in large and irregular-shaped room. Statistical distribution of acoustical field using a four-microphone configuration implemented in a previous study (M. W. Budhiantho, Acoustic Velocity Related Distributions dissertation, The University of Texas at Austin, August 1997) has been extended to a seven-microphone configuration in this report. An effort has been made to model acoustical field near the boundaries of an enclosed space (reverberation room). Also 3-D imaging technique of acoustic intensity is also presented in this report.
10:35

3aSP8. Acoustic source localization with eye array. Hedayat Alghassi, Shahram Tafazoli, and Peter Lawrence (Dept. of Elec. and Comput. Eng., University of British Columbia, Vancouver, BC V6T 1Z4, Canada, hedayata@ece.ubc.ca)

A novel signal-processing algorithm and array for sound source localization (SSL) in three-dimensional space is presented. This method, which has similarity to the eye in localization of light rays, consists of a novel hemispherical microphone array with 26 microphones on the shell and one microphone in the sphere center. The microphones on the shell map a geodesic hemisphere called two-frequency icosahedron; hence, each microphone has at least four other orthogonal microphones. A signalprocessing scheme utilizes parallel creation of a special closeness function for each microphone direction on the shell in the time domain. Each closeness function cell (lens cell) consists of center microphone, shell microphone, and one of its orthogonals. The closeness function output values are linearly proportional to spatial angular difference between the sound source direction and each of the shell microphone directions. By choosing microphone directions corresponding to the highest closeness function values and implementing a linear weighted spatial averaging on them, the sound source direction is estimated. Contrary to traditional SSL techniques, this method is based on simple parallel mathematical calculations 
in the time domain with low computational costs. The laboratory implementation of the array and algorithm shows reasonable accuracy in a reverberant room.

\section{0:50}

3aSP9. Bayesian space-time processing for acoustic array source estimation using a towed array. James V Candy (Lawrence Livermore Natt. Lab., Univ. of California, P.O. Box 808, L-156, Livermore, CA 94551) and Simon J. Godsill (Univ. of Cambridge, Cambridge CB2 1PZ, UK)

The development of a model-based Bayesian processor for a horizontally towed array using synthesized narrow-band, spatio-temporal data is discussed. The basic processor is developed from the Bayesian perspective, capturing the bearing estimation problem of acoustic sources moving in a surveillance volume. Far-field sources characterized by simplified random motion as well as nonlinear measurement models representing the array motion and propagation of the source signatures contaminated by noise are incorporated into the processor. The entire dynamical system is cast within a Bayesian framework aimed at estimating the underlying posterior filtering distribution of the bearing in a pseudo real-time environment. It is shown that the resulting sequential Bayesian processor is capable of dynamically estimating the instantaneous posterior distribution enabling the extraction of the bearing estimates. The problem solution involves the estimation of the posterior using sequential importance sampling/resampling leading to the development of a particle filter for this problem. The particle filter is simply an empirical representation of the posterior characterized by the weights (probability) and location variables (particles). It is shown how the particle filter successfully extracts the desired signals comparing the results to classical nonlinear processors (e.g., extended/unscented Kalman filters) for this problem.

\section{1:05}

3aSP10. A design of array-microphone system using pressure-gradient microphones and two-dimensional finite impulse response filters. Hiroyuki Naono (Matsushita Tech. Information Services Co., Ltd., 25-10 Nishiyama Adachi Yawata city, Kyoto 614-8351 Japan, yq107401@nifty.ne.jp) and Kiyoshi Nishikawa (Kanazawa Univ., Kakuma, Kanazawa-shi, 920-1192 Japan)

Delay-and-sum array microphones consisting of a linear array of microphones can achieve sharp directivity at high frequencies. A disadvantage is that the directivity is frequencydependent. An array microphone using a fan filter is proposed to resolve that problem. However, it is necessary to increase the filter order to form a sharp beam at a low frequency. This paper is reported improvement of the array microphone directivity, realizing a narrow beam with a low order of the filter using a twodimensional frequency domain design. Using four microphones, a secondorder pressure-gradient microphone is formed. The spacing of the microphones is set for high sensitivity and broad bandwidth in the operating frequency range. These microphone units are placed along a line and are cascaded to a two-dimensional fan filter so that the load is distributed. In the proposed configuration, the second-order pressure-gradient microphone is cascaded with an axially symmetric fan filter. The configuration thereby forms a single broadside beam. Consequently, the directional characteristics of the pressure-gradient microphone are reflected in the array microphone and a sharp directivity, which is frequencyindependent, is obtained over a wide frequency range.

\section{1:20}

3aSP11. Pressure-sensitive paint as a distributed optical microphone array. James W. Gregory ${ }^{a}$, John P. Sullivan (School of Aeronautics and Astronautics, Purdue Univ., 315 N. Grant St., West Lafayette, IN 47907, jim.gregory@alumni.purdue.edu), Sameh S. Wanis, and Narayanan M. Komerath (Georgia Inst. of Technol., Atlanta, GA 30332)

Pressure-sensitive paint is presented and evaluated in this paper as a quantitative technique for measurement of acoustic pressure fluctuations. This work is the culmination of advances in paint technology, which enable unsteady measurements of fluctuations over $10 \mathrm{kHz}$ at pressure levels as low as $125 \mathrm{~dB}$. Pressure-sensitive paint may be thought of as a nanoscale array of optical microphones with a spatial resolution limited primarily by the resolution of the imaging device. Thus, pressure-sensitive paint is a powerful tool for making high-amplitude sound pressure measurements. In this work, the paint was used to record ensemble-averaged, time-resolved, quantitative measurements of two-dimensional mode shapes in an acoustic resonance cavity. A wall-mounted speaker generated nonlinear, standing acoustic waves in a rigid enclosure measuring $216 \mathrm{~mm}$ wide, $169 \mathrm{~mm}$ high, and $102 \mathrm{~mm}$ deep. The paint recorded the acoustic surface pressures of the $(1,1,0)$ mode shape at $1.3 \mathrm{kHz}$ and a sound pressure level of $145.4 \mathrm{~dB}$. Results from the paint are compared with data from a Kulite pressure transducer and with linear acoustic theory. The paint may be used as a diagnostic technique for ultrasonic tests where high spatial resolution is essential, or in nonlinear acoustic applications such as shock tubes. ${ }^{\text {a) }}$ Currently at the Dept. of Aeronautics, U.S. Air Force Academy.

\section{$11: 35$}

3aSP12. Performance of an infrasound source localization algorithm. Curt Szuberla, Kenneth Arnoult, and John Olson (Geophysical Inst., Univ. of Alaska Fairbanks, 903 Koyukuk Dr., Fairbanks, AK 99775-7320)

We present a performance analysis of a method of acoustic source localization based on time difference of arrival (TDOA) information for an arbitrary array of sensors. The method begins with the construction of a vector containing estimates of time delays for each unique sensor pair in the array via cross correlation. An optimization in the space of geographic location and sound speed is then conducted to minimize the difference between the observed vector and one calculated from a search in that space. The technique uses an analytic method of seeding based on the acoustic analog of a light cone. The source localization procedure is tested as a function of a number of parameters, including array aperture, number of sensors, sample rate, signal-to-noise ratio, and signal type. The technique is shown in application to real and synthetic infrasound signals and its statistical behavior is given. 


\title{
Session 3aUWa
}

\section{Underwater Acoustics and Acoustical Oceanography: Sediment Acoustic Processes: Quantifying the Effects of Sediment Properties on Dispersion, Attenuation, Reflection, Scattering, and Buried Target Detection III}

\author{
Eric I. Thorsos, Cochair \\ Univ. of Washington, Applied Physics Lab., 1013 NE 40th St., Seattle, WA 98105-6606 \\ Masao Kimura, Cochair \\ Tokai Univ., Dept. of Geo-Environmental Technology, 3-20-1 Shimizu-Orido, Shizuoka 424-8610, Japan
}

Contributed Papers

$7: 45$

3aUWa1. Seafloor manipulative experiments during sediment acoustics experiments (SAX99 and SAX04. Michael D. Richardson (Marine Geosciences Div., Naval Res. Lab., Stennis Space Center, MS 39529-5004) and Kevin L. Williams (Univ. of Washington, Seattle, WA 98105-6698)

Seafloor manipulations were performed during Sediment Acoustics Experiments (SAX99 and SAX04) in an attempt to test predictions of high-frequency acoustic backscattering models in sandy environments. Manipulations included alteration of seafloor roughness by raking to mimic the effects of sand ripples on acoustic scattering and placement of marbles, aluminum disks, and shells on the seafloor at different densities to mimic the effects of shelled animals and sand dollars on acoustic scattering (discrete surface scattering). In general, the predictions from scattering models and measured acoustic backscatter strengths were in agreement and the experimental results provide insight into natural phenomena. [Work supported by ONR.]

\section{8:00}

3aUWa2. A simple model for time-dependent scattering by changing seafloor roughness. Darrell R. Jackson, Kevin L. Williams, Christopher D. Jones (Appl. Phys. Lab., Univ. of Washington, 1013 NE 40th St., Seattle, WA 98105-6698, drj@apl.washington.edu), Michael D. Richardson, Kevin B. Briggs (Naval Res. Lab., Stennis Space Center, MS 39529-5004), and Anthony P. Lyons (Appl. Res. Lab., State College, PA 16804-0030)

The roughness of sandy seafloors is constantly changing due to biological and hydrodynamic activity. Even when these processes are in equilibrium so that the average acoustic backscattering intensity does not change, the character of successive backscatter echoes changes on time scales from minutes to days, with strong frequency dependence. This change can be characterized by the temporal correlation of the backscattered signal between consecutive pings, expressed as a lag-dependent correlation coefficient. A simple model for roughness change has been developed based upon the diffusion equation. This model assumes that newly created roughness is uncorrelated with existing roughness. It predicts exponential decay of the correlation with e-folding time $1 /\left(D K_{B}^{2}\right)$, where $K_{B}$ is the Bragg wave number, and $D$ is the horizontal diffusivity. During SAX99 and SAX04, photographic and acoustic observations were made of changing natural and artificially generated roughness. The acoustic observations were made at 40 and $300 \mathrm{kHz}$, with the higher frequency exhibiting much more rapid decorrelation as predicted by the model. The diffusivities obtained by fitting the model to photographic and acoustic data ranged from roughly $10^{-9}$ to $10^{-10} \mathrm{~m}^{2} \mathrm{~s}^{-1}$ with the highest diffusivities found at locations where bottom-feeding fish were active. [Work funded by ONR.]
8:15

3aUWa3. Using forward scattering from a rippled sand/water interface to determine its flat surface reflection coefficient: Validation via simulation and use in testing sediment models. Kevin Williams (Appl. Phys. Lab., Univ. of Washington, 1013 NE 40th St., Seattle, WA 98105-6698,williams@apl.washington.edu)

Real-world sediments are inherently rough. At high frequencies (greater than $10 \mathrm{kHz}$ ) the roughness is typically sufficient to eliminate any coherent reflection at all but very shallow grazing angles. However, simulation results will be shown that demonstrate that an ensemble average of a large number $[O(50)]$ of forward-scattered signals from different regions of a rippled sand/water interface can be used to estimate the flat surface reflection coefficient for the interface. With this validation in hand, a forward scattering experiment carried out during a sediment acoustics experiment in 2004 (SAX04) will be described. The flat surface reflection coefficients derived from this experiment will be compared with predictions using a fluid and a Biot model for the sand sediment. This comparison indicates that a Biot sediment model is a more accurate predictor for the reflection coefficient. [Work supported by ONR.]

\section{8:30}

3aUWa4. High-resolution mapping and backscatter studies in support of the Ripples Program. Barbara Kraft, Christian de Moustier, Brian Calder, and Larry Mayer (Ctr. for Coastal and Ocean Mapping, Univ. of New Hampshire, 24 Colovos Rd., Durham, NH 03824, bjkraft@ccom.unh.edu)

The long-term goal of the Office of Naval Research's RIPPLES DRI is to develop the ability to predict the geometry and evolution of seabed morphology, particularly the presence or absence of ripples which have been shown to affect the sub-seabed penetration of acoustic energy. In support of the RIPPLES and SAX04 programs, high-resolution, multifrequency, multibeam mapping surveys were completed during September and October 2004 within three small areas off Destin, FL. The first survey was completed prior to Hurricane Ivan and followed the burial of minelike targets in the SAX04 field area. The second survey occurred during the SAX04 experiments approximately 6 weeks after Hurricane Ivan made landfall. Ripples were not observed in the bathymetric data from the first survey. Some small-scale features are present in the bathymetry from the second survey; however, most of the ripples generated by Hurricane Ivan had degraded by the time the survey was completed. Large-scale ripple features are present in the bathymetric data that extend off-shore from the SAX04 area. Difference maps of the pre- and post-Hurricane Ivan bathymetry and the acoustic backscatter data show remarkable evidence of sediment deposition and erosion. [Work supported by ONR Grant N00014-041-0624.] 
3aUWa5. Doppler spreading of aircraft harmonics in a shallow-water channel off Kauai. David Barclay and Michael J. Buckingham (Marine Physical Lab., Scripps Inst. of Oceanogr., UCSD, 9500 Gilman Dr., La Jolla, CA 02093-0238)

In September 2005, during the Makai experiments off Kauai, a light aircraft acted as a moving sound source over a shallow-ocean channel with a coral-sand bottom. Core samples yielded the porosity, bulk density, grain density, and grain size of the sediment. Acoustic sensors (a microphone in the air and a line of hydrophones in the isovelocity water column) recorded the sound of the aircraft as it flew over the sensor station. At least five engine/propeller harmonics, from 80 to $400 \mathrm{~Hz}$, are present in the spectra of the hydrophone signals. Each harmonic exhibits Doppler spreading, with well-defined spectral peaks appearing on either side of the unshifted frequency. Those peaks with the greatest (up- and down-) shifts are associated with the shallow equivalent rays of normal modes in the water column. Two head-wave peaks, associated with slightly steeper rays, are less up- and down-shifted. Least shifted of all, because of their steep equivalent rays, are peaks due to modes supported by the sea surface and the subbottom, a few meters beneath the seabed. The spectral structure in the Doppler-spread harmonics is the basis of Doppler spectroscopy, an inversion technique for recovering the geoacoustic parameters of the seabed. [Research supported by ONR.]

9:00

3aUWa6. Using buried vector sensors to examine seabed layering in sandy sediment. Paul C. Hines, John C. Osler, Jeff Scrutton, David M.F. Chapman (Defence R\&D Canada Atlantic, P.O. Box 1012, Dartmouth, NS, Canada, B2Y 3Z7), and Anthony Lyons (The Penn State Univ., State College, PA 16804-0030)

There is considerable interest within the underwater acoustics community in quantifying the sound-speed dispersion in sandy sediments at low kiloHertz frequencies. To address this, data were collected during the SAX04 experiment (a US Office of Naval Research Departmental Research Initiative) using projectors and directional (vector) receivers buried in the top $1 \mathrm{~m}$ of the seabed, in conjunction with a water-borne source directly above the buried sensor field. Although using buried sensors significantly increased the complexity of the experimental setup, it should enable a direct time-of-flight measurement of acoustic wave speed, which simplifies interpretation of the results. However, initial analysis of the time-of-flight results indicates the existence of a buried layer, which increases the complexity of the data. In this paper, the vector sensors are used to steer beams to confirm the existence of the layer and to isolate the direct and reflected arrivals to improve the estimate of sound speed dispersion. [Work partially funded by US Office of Naval Research.]
3aUWa7. Estimation of received pulse from sea bottom with a transition layer using elastic finite difference time domain method. Takenobu Tsuchiya and Nobuyuki Endoh (Faculty of Eng., Kanagawa Univ., 3-27, Rokkakubashi, Kanagawa-ku, Yokohama, Japan)

Recently, ocean acoustic thermometry is being planned in shallow water. Characteristics of sound propagation in shallow water are influenced by bottom media because sound propagation is affected by acoustic boundary conditions of the water-sediment interface. To investigate characteristics of sound propagation in shallow water, it is necessary to develop an accurate numerical method to model sound propagation. Elastic finite difference time domain method (elastic FDTD) is very accurate and is useful for range-dependent models in shallow water. Therefore, we calculate the sound waveform reflected from the sea bottom with a transition layer in shallow water using elastic FDTD. It is assumed that a $1.5-\mathrm{kHz}$ transducer is placed at 50-m depth. The influence of the sea bottom with the transition layer is calculated for a 400-m range. To determine the influence of thickness of the transition layer to sound propagation, we calculate sound pulses as transition layers of varying thickness. Results show that fluctuation of the pulse amplitude depends on the transition layer thickness if the thickness is greater than one wavelength. A sea bottom with a transition layer clearly affects sound propagation in shallow water.

\section{9:30}

3aUWa8. Stability of compressional waves in granular media. B. Edward McDonald (Naval Res. Lab., Washington, DC 20375)

Nonlinear compressional waves in a granular medium such as the seafloor are subject to the Hertzian nonlinearity of order $3 / 2$ in the strain rate. As a result, the coefficient of quadratic nonlinearity becomes arbitrarily large at low stress. Two theoretical problems result from the Hertzian nonlinearity: (1) lowest order nonlinearity in the equation of state is no longer quadratic and (2) questions of stability arise when nonlinear steepening occurs arbitrarily fast. A variant of the NPE [McDonald and Kuperman, J. Acoust. Soc. Am. 81, 1406-1417 (1987)] is used to derive a stability theorem for self-similar waves in a granular medium subject to three-dimensional perturbations. When the dominant nonlinearity is of order $n$ between 1 and 3 ( $n$ is not necessarily an integer), the result suggests that wave stability is positively correlated with $n$. The method of characteristics is used to show that where the coefficient of nonlinearity diverges, the wave slope is forced to zero. [Work supported by the Office of Naval Research.] 


\title{
Session 3aUWb
}

\section{Underwater Acoustics: Sound Propagation: Measurements and Modeling I}

\author{
Martin Siderius, Cochair \\ Heat Light \& Sound Research Inc., 12730 High Bluff Dr., Ste. 130, San Diego, CA 92130 \\ Nobuyuki Endoh, Cochair \\ Kanagawa Univ., Dept. of Engineering, 3-27-1 Rokka Kubashi, Yokohama 221-8686, Japan
}

Chair's Introduction-9:55

\section{Contributed Papers}

\section{0:00}

3aUWb1. Benchmarking range-dependent seismo-acoustic propagation problems. Peter L. Nielsen, Finn B. Jensen, Mario Zampolli (NATO Undersea Res. Ctr., 19138 La Spezia, Italy), Michael D. Collins (Naval Res. Lab., Washington, DC 20375), and William L. Siegmann (Rensselaer Polytechnic Inst., Troy, NY 12180)

Developing the capability to accurately solve range-dependent problems in ocean acoustics has been going on for many years. A milestone in this development was the ASA benchmark effort in 1990 [J. Acoust. Soc. Am. 87, 1497-1545 (1990)], where a set of models was applied to generic range-dependent acoustic fluid problems to establish a set of benchmark solutions for model verification purposes. A further benchmarking effort, which included shear waves in the bottom, was published in 1997 [IEEE J. Ocean. Engn. 22, 226-236 (1997)] involving two seismo-acoustic codes based on spectral-integral techniques and a boundary-element code. Other codes have become available since then for solving general rangedependent elastic problems, either based on marching schemes that ignore backscattering (or adopt a single-scatter approximation) or techniques that solve the full boundary-value problem. In an attempt to establish a set of benchmark solutions for seismo-acoustic problems, we solve several lowfrequency elastic problems involving changing bathymetry in multiple layers, and coupling between various wave types, including interface waves. The models in use or used are a parabolic equation model (RAM) and a commercial finite-element model (FEMLAB).

\section{0:15}

3aUWb2. Bathymetric and oceanic variability effects on acoustic propagation metrics in a shelf-break environment. Roger Oba (Acoust. Div., Naval Res. Lab., Washington, DC 20375)

The interaction of oceanographic variability and bathymetry on acoustic propagation in the littoral is quite complex, and the relative importance is not readily quantified, especially for data-model comparisons. Sound speeds derived from ocean models could be compared directly to experimental sound-speed data, but this is a crude measure of environmental impact on acoustics. Instead, a 300-Hz source in a three-dimensional parabolic equation (PE) acoustic propagation model provides input to proposed acoustic metrics, especially related to array performance, to assess the effects of experimentally derived bathymetry and tide. The metrics can also be used for comparison of an ocean model and experimentally derived sound-speed profiles. Other acoustic mode metrics can be derived and compared to the more computationally intensive three-dimensional PE derived measures. As an example, the Navy Coastal Ocean Model (NCOM), a mesoscale oceanographic model, predicts sound-speed gridded on the order of $5 \mathrm{~m}$ in depth and $200 \mathrm{~m}$ in range at 6-h updates for the ASIAEx South China Sea site. This can then be used for metric calculations and data-model comparison. [This research is sponsored by the ONR.]
10:30

3aUWb3. Adaptive predictive-error optimal gridding. Donald $\mathrm{R}$. DelBalzo and Lisa A. Pflug (Planning Systems Inc., 40201 Hwy. 190 E, Slidell, LA 70461)

Transmission loss computations in littoral areas often require a dense spatial and azimuthal grid to achieve acceptable accuracy and detail. The computational cost of accurate predictions in complex areas can be unacceptable for some Naval applications. EAGLE (Efficient Adaptive Gridder for Littoral Environments) was developed to reduce the computational requirements by adapting the fundamental calculation grid to the natural environmental structure. EAGLE produces a nonuniform grid structure within which the underlying data have approximately constant spatial uncertainty, or iso-deviance. The EAGLE point-selection approach is based on a predictive-error metric and least-squares polynomial curve fitting with arbitrary order. This research indicates the degree to which EAGLE grids are related to the underlying coherence properties of the natural environment. The results show significant efficiency enhancements compared to uniform grids of the same accuracy, when the environmental coherence properties are used to control the polynomial order. [Work sponsored by NAVAIR.]

\section{0:45}

3aUWb4. The effect of an ocean front on sound propagation in shallow water. Ying-Tsong Lin, Arthur E. Newhall, and James F. Lynch (Woods Hole Oceanogr. Inst., Woods Hole, MA, 02543, ytlin@whoi.edu)

From a perspective of sound propagation, a sloping bottom found in typical shallow-water environments can cause propagating sound to horizontally refract offshore. Additionally, an offshore ocean front can cause horizontal reflection/refraction shoreward. Combining these two effects, sound tends to propagate along the front, and "whispering gallery" modes can be observed. To model this effect, we first derived an analytic solution for the sound field using a point source in a rigid wedge environment that has a front placed offshore. The analytic solution gives us clear physical insight into this problem. For a more complicated environment, we exploited a 3-D acoustic propagation program in the ACOUSTICS TOOLBOX, which uses a vertical mode and horizontal Gaussian beam method. This model shows sound energy trapped ahead of the front with observable frequency and modal dependence. Also, when including the foot of the front, which is commonly seen on the continental shelf and extends inshore along the bottom, the model shows less modal attenuation, which raises the level of the trapped energy. 
3aUWb5. Asymptotic expansions for normal modes in transparent wedge. Nick Maltsev (Guzuk Tech. Enterprises, 2443 Wyandotte St., Mountain View, CA 94043)

Asymptotic solutions of exact infinite system of equations for coefficients of angular Fourier harmonics of sound field have been built and computed for an arbitrary transparent wedge. Asymptotic solutions are compared with exact field in the case of two half-spaces and a rectangular wedge.
11:15

3aUWb6. Polynomial chaos expansions as extended Markov approximations in the propagation of uncertainty. Dennis Creamer (P.O. Box 660537, Arcadia, CA 91066)

The use of polynomial chaos for incorporating environmental variability into propagation models has been previously investigated where it was found that low-order expansions work quite well when the medium correlation length in the direction of propagation was small, i.e., the Markov approximation is roughly valid. It was suggested that an iterative scheme incorporating extensions of the Markov approximation might be useful. Using the method of successive approximations introduced by Klyatskin and Tatarskii (who used the method for investigating the validity of the Markov approximation), we show how the polynomial chaos equations can be written as a (smaller) set of equations that are basically nested expansions in the correlation function. This allows the use of a closure scheme, which does not involve truncating the expansion and which seems to be simpler to use. Comparisons are made between the approximation schemes in a geometrical optics limit.
3aUWb7. Polynomial chaos expansions for travel time and amplitude uncertainty in acoustic time series in ocean waveguides. Kevin D. LePage (Naval Res. Lab., Code 7144, 4555 Overlook Ave SW, Washington, DC 20375)

Polynomial chaos (PC) techniques have shown themselves to be a powerful technique for the estimation of the statistical properties of ocean acoustic propagation for situations where the vertical and horizontal structure of sound speed uncertainty can be specified in terms of empirical orthogonal functions (EOFs) with known amplitude probability distribution functions. Here we extend the $\mathrm{CW}$ solutions for the statistical properties of the normal models describing the acoustic field in the presence of internal waves with Gaussian distributed EOF descriptions to the time domain to describe the scintillation of the acoustic time series. Results are compared to exact solutions as well as to estimates based on the adiabatic approximation. Results show that the PC solutions do a much better job of capturing the true variability of the exact solutions for the time series variability than the adiabatic approximations does. [Work supported by ONR.]

\section{1:45}

3aUWb8. The propagation of acoustic field uncertainty in an ocean waveguide having finite correlation length sound speed variations. Steven Finette (Acoust. Div., Naval Res. Lab., Washington, DC 20375)

While the physical laws governing wave propagation in ocean waveguides are well understood, the appropriate wave equations typically depend on parameters, fields, boundary, and initial conditions that are, at best, only partially known in any given environment. Incomplete environmental knowledge concerning these quantities is a form of uncertainty that should be characterized and quantified in any simulation-based prediction scheme involving the acoustic field. This talk focuses on the quantification of uncertainty in ocean waveguide propagation using a probabilistic formulation in which both the sound speed and acoustic fields are represented by stochastic basis expansions. An example is discussed for the case of an ocean waveguide with separable depth and range correlation functions describing the sound speed field. The relationship between this example and the propagation of acoustic field uncertainty in an internal wave field is discussed. [Work supported by ONR.] 


\title{
Session 3pAAa
}

\section{Architectural Acoustics and Noise: Acoustic Design for Green Buildings}

\author{
Kenneth P. Roy, Cochair \\ Armstrong World Industries, Innovation Ctr., 2500 Columbia Ave., Lancaster, PA 17604 \\ Tetsuya Sakuma, Cochair \\ Univ. of Tokyo, Graduate School of Frontier Science, Environmental Bldg. 627, 5-1-5 Kashiwanoha, Kashiwa-shi, \\ Chiba 277-8563 Japan
}

Chair's Introduction-1:00

Invited Papers

1:05

3pAAa1. An overview of Leadership in Energy and Environmental Design (LEED) certification and acoustical design: Conflicts and synergies. Daniel C. Bruck (BRC Acoust. \& Technol. Consulting, 1741 First Ave. South, Ste. 401, Seattle, WA 98134), David Lubman (14301 Middletown Ln., Westminster, CA 92683), and Louis C. Sutherland (27803 Longhill Dr., Palos Verdes, CA 90275)

The LEED program for green building certification acknowledges the importance of acoustics in building quality and design, but currently does not provide for specific credits in acoustics other than in the Innovation in Design category. This paper presents an overview of the LEED approach to sustainable design and discusses the design categories for LEED certification. Conflicts and synergies between the LEED system and acoustical design are discussed, including specific areas in which acoustics can contribute to sustainable design goals, such as recycled materials, energy efficiency, and interior environmental quality. Steps toward integrating acoustics into the LEED system are proposed, which incorporate elements of the Collaborative for High Performance Schools (CHPS) and ANSI S12.60, Acoustical Performance Criteria, Design Requirements, and Guidelines for Schools. The current direction of the U.S. Green Building Council with respect to incorporating acoustics into the LEED program is also presented.

\section{$1: 25$}

3pAAa2. Acoustical lessons from four green buildings. Max Richter, Rosamund Hyde (Stantec, 1100-111 Dunsmuir St. Vancouver, BC V6B 6A3, Canada), and Murray Hodgson (University of British Columbia, V6T 1Z3, Canada)

Between 2002-2004, seven green buildings in British Columbia were studied during a Post Occupancy Evaluation (POE) research effort led by Stantec (formerly KEEN Engineering). Using the occupant satisfaction were survey developed by the Center for the Building Environment at UC Berkeley, data on eight aspects of occupant satisfaction were collected, including acoustical satisfaction. The BC buildings generally followed the trends that can be observed in green building acoustical performance, but none of the buildings scored as satisfactory. This year, Stantec and UBC measured four of the building in the original study to determine the acoustical characteristics of the building spaces. From this subsequent study, conclusions can be drawn about the relative acoustical performance of specific green strategies.

\section{$1: 45$}

3pAAa3. Acoustical qualities of three Leadership in Energy and Environmental Design (LEED) certified buildings and why some disappoint. Steven D. Pettyjohn (The Acoust. \& Vib. Group, 5700 Broadway, Sacramento, CA 95820-1852)

Obtaining points for acoustical qualities in office building or schools is difficult under the current LEED system. For this reason, the selection of partitions to provide speech privacy or prevent speech interference, the design of HVAC systems to meet background sound or vibration goals, or the inclusion of acoustical materials to provide the desired reverberation time is often either left out from the beginning or the first to be cut when budgets are tight. The results of sound tests done as a part of the commissioning of two office complexes and a new combination school, learning center, and administration building are shared. NIC tests were done on fixed and operable wall assemblies, background sound levels were measured in various spaces when due to mechanical and electrical systems and exterior sources, and reverberation time tests were done in conference rooms, classrooms, offices, and auditoria. The reason design goals were not met in two of the three complexes is related to budget cuts and a misunderstanding of the requirements and their importance to the successful use of the facilities. The commissioning process failed to correct the deficiencies. 
3pAAa4. Acoustical evaluation of six "green" office buildings. Murray Hodgson (Acoust. \& Noise Res. Group, SOEH-MECH, University of British Columbia, 3rd Fl., 2206 East Mall, Vancouver, BC V6T 1Z3, Canada), Rosamund Hyde, Blair Fulton, and Max Richter (Stantec Consulting Ltd., Vancouver, BC V6B 6A3, Canada)

As part of a project evaluating the performance of six "green" office buildings, detailed acoustical measurements were made to explain the positive and negative comments of building occupants obtained from a questionnaire survey. The objective was to determine how to design better green buildings. This paper presents an overview of the measurement protocal used, the measurements made, the results, and the lessons learned regarding optimal design.

3pAAa5. Acoustical evaluation of a "green" office building. Murray Hodgson (Acoust. \& Noise Res. Group, SOEH-MECH, University of British Columbia, 3rd Fl., 2206 East Mall, Vancouver, BC V6T 1Z3 Canada), Rosamund Hyde, Blair Fulton, and Max Richter (Stantec Consulting Ltd., Vancouver, BC V6B 6A3, Canada)

As part of a project evaluating the performance of six "green" office buildings, detailed acoustical measurements were made on three floors of an office building in order to explain the positive and negative comments of building occupants obtained by a questionnaire survey. The objective was to determine how to design better green buildings. The building contained public spaces, private/closed offices, and open-plan-office areas. This paper presents details of the measurements performed, the results, and the design lessons learned.

\section{2:45}

3pAAa6. An overview of sound environmental aspect of Japan's comprehensive assessment system for building environmental efficiency (CASBEE) certifications. Tetsuya Sakuma and Fumiko Yano (Grad. School of Frontier Sci., The Univ. of Tokyo, 5-1-5 Kashiwanoha, Kashiwa-shi, Chiba 277-8563, Japan, sakuma@k.u-tokyo.ac.jp)

In the worldwide trend towards sustainable buildings, a new system called the Comprehensive Assessment System for Building Environmental Efficiency (CASBEE) has been established in Japan, as well as other existing assessment systems such as BREEM, LEED, GB Tool, etc. In particular, CASBEE is intended to evaluate building performance by a single-number index BEE, which is the ratio of environmental qualities to loadings. Concerning sound environment, three items are included in indoor quality evaluation: (1) noise (background noise, equipment noise); (2) insulation (window opening, wall, heavy/light floor impact sound); and (3) absorption; in addition, one item on noise emission outside the building site is included in environmental loading evaluation. However, there are many unclear points on the settings of items, ratings, and weightings, which will make trouble in practical application. In the last 3 years, several large cities have started a certification system using CASBEE for new large-scale buildings. In this presentation, an overview of the assessment system and the statistics of the city of Yokohama will be presented focusing on sound environmental aspect.

$$
\text { 3:05-3:25 Break }
$$

3pAAa7. Achieving acoustical satisfaction in a green building. Ethan Salter, Charles M. Salter (Charles M. Salter Assoc., Inc., 130 Sutter St., Ste. 500, San Francisco, CA 94104), Chuck Davis, and Scott Shell (EHDD Architecture, San Francisco, CA 94110)

The Carnegie Institution's Global Ecology Research Center at Stanford University has garnered many accolades, including the AIA's Excellence in Sustainability Award. This building incorporates many "green" and energy-saving design features, mechanical systems, and materials. The occupants of this facility have given it high marks in U.C. Berkeley's Center for the Built Environment's (CBE) survey of buildings. Staff at the Global Ecology Research Center are shown to be more satisfied with their acoustical environment than occupants of other green buildings surveyed by CBE. Measured acoustical data for speech privacy in open plan and enclosed conference rooms will be presented along with descriptions of acoustical design attributes for the building.

\section{$3: 45$}

3pAAa8. Relationship between ventilation, air quality, and acoustics in "green" and "brown" buildings. Alireza Khaleghi, Karen Bartlett, and Murray Hodgson (SOEH-MECH, University of British Columbia, 3rd Fl., 2206 East Mall, Vancouver, BC V6T 1Z3, Canada)

This paper discusses a project involving direct monitoring of ventilation, indoor-air quality, and acoustical quality in "green" and "brown" buildings on the UBC campus. The objective was to determine the relationship between these various environmental factors, and the implications of the results for the ventilation-system concept/design, especially in green buildings. The buildings studied, the measurements performed, and the main results are described. The lessons learned about ventilation design are discussed. 
3pAAa9. Acoustic and thermal evaluation low-cost dwelling, a case study in Curitiba, Brazil. Paulo H. Zannin (Mech. Eng. Dept., Federal Univ. of Parana, Rua Benedicto Berillo Fangueiro, 108, Santa Barbara, Curitiba, Parana, CEP, 81540-420, Brazil) and Eduardo Krueger (Cefet of Parana, Rebouas, Curitiba, PR)

The present paper presents acoustic and thermal performance evaluations carried out in low-cost houses located at the Technological Village of Curitiba. Two dwellings were evaluated, which were built according to different building systems: (1) the building system Batistella, built with prefabricated modules and elements, composed of reforestation wood, and (2) the building system Constroyer, composed of EPS plates, framed in a welded iron mesh, with structural plastering. For the thermal performance evaluation, it was verified that the materials used in the envelope were quite significant for controlling heat gains and losses through the building elements as well as for regulating heat storage. In this case, the system with highest thermal inertia presented better results. Results showed that both building systems had poor acoustic performance, characterized by a low weighted apparent sound reduction index of the facade $R^{\prime} w=18 \mathrm{~dB}$. The German DIN 4109 Norm recommends $R^{\prime} w=30 \mathrm{~dB}$. The weighted apparent sound reduction index measured for internal walls was $R^{\prime} w$ $=25 \mathrm{~dB}$ and $R^{\prime} w=30 \mathrm{~dB}$, for the building systems Battistela and Constroyer, respectively. The minimum required by German VDI 4100 .
3pAAa10. Indoor occupant productivity perception and environmental sound measurement correlation. Brian J. Landsbeger, Liangcai Tan, and Xin Hu (Dept. of Mech. Eng., UNLV, 4505 Maryland Pkway, Las Vegas, NV 89154)

Occupant comfort and productivity have gained increasing attention by building owners and managers in comparison with traditional concerns about costs associated with building function and operating efficiency. To properly assess building performance, a comprehensive method for measurement and verification of integrated building performance was developed. Indoor sound, temperature, air movement, humidity, lighting, and air quality were measured for 3 days each in ten office buildings and ten schools. Concurrently, a perception questionnaire was completed by a subset of the occupants in the buildings and schools. The resulting questionnaire data were correlated with the physical measurements to determine what measures or combination of measures most accurately indicate worker perception of productivity variation when working under various environmental conditions. This presentation concentrates on correlation of acoustic measures, including $\mathrm{dBA}, \mathrm{NC}, \mathrm{NCB}, \mathrm{RC}$, and RC Mark II to worker perception of noise effects on productivity variation. Also, worker perception of productivity variation due to all measured indoor environmental factors was examined to determine additive effects and interaction between noise and other environmental factors. An ultimate goal of the study is to produce a method to quantitatively determine expected worker productivity variation based on measurable environmental factors.

THURSDAY AFTERNOON, 30 NOVEMBER 2006

OAHU ROOM, 1:00 TO 3:20 P.M.

\title{
Session 3pAAb
}

\section{Architectural Acoustics: Sound Diffusion, Reflection, and Absorption}

\author{
Jason E. Summers, Cochair \\ Naval Research Lab., 4555 Overlook Ave., S.W., Washington, DC 20375 \\ Toru Otsuru, Cochair \\ Oita Univ., Faculty of Engineering, 700 Dannoharu, Oita 870-1192, Japan
}

Chair's Introduction-1:00

\section{Contributed Papers}

1:05

3pAAb1. Design of the diffuser with hemispheres using 1:10 and real scale models. Shin-ichi Sato and Jin Yong Jeon (School of Architectural Eng., Hanyang Univ., Seoul 133-791, Korea, s_sato@mac.com)

Sound diffusion by a wall structure is one of the main concerns with respect to the sound quality of concert halls. To quantify the sound diffusion of an actual hall from in situ measurements, the relationship between the scattering coefficient and the acoustical parameters measured in the laboratory should be investigated first. In this study, the scattering coefficient and acoustical parameters of the diffusers with hemisphere were investigated. The diameter, density, and the arrangement pattern of the hemisphere were investigated in terms of the scattering coefficient and the parameters obtained from the impulse response including temporal diffusion [H. Kuttruff, Room Acoustics (Elsevier Science, London, 1991)]. The results of measurements in 1:10 scale reverberation chamber with the same scale diffusers were verified by real scale results. Sound scattering index for reverberation chamber, which quantifies the scattered sound field, was proposed. The optimum condition of the hemisphere diffuser specification maximizing the effect of scattering on a sound field was also investigated.

\section{$1: 20$}

3pAAb2. Augmented-reality virtual-diffusion system: A tool to evaluate listener comfort. Pedro Novo (Acoust. Res. Ctr., Univ. of Salford, M5 4WT, UK, p.novo@salford.ac.uk)

The augmented-reality virtual-diffusion system presented here aims to evaluate the comfort of a subject listening to a nearby speaker located in a room filled with a diffuse crowd. The main parameter to be studied is sound field diffusion and its relation to comfort. To this purpose a virtual diffusion system, based on electro acoustic transducers mounted on the walls, is employed to actively change the properties of the diffuse sound field. The augmented reality factor is introduced through the interaction of the virtual diffusion system with the acoustics of the room. The present paper presents a brief description of the approach chosen to implement the augmented-reality virtual-diffusion system. Diffusion measurements obtained with both virtual and real diffusers are compared. Results regarding 
auditory events location evoked by different sound fields and predictions from a cross-correlation-based binaural model employed to model these results are discussed. Initial results relative to comfort evoked by the different sound fields as well as a possible relation between auditory events location and listener comfort are discussed.

\section{$1: 35$}

3pAAb3. Simulation of in situ measurement of diffusion and scattering coefficients. Diemer de Vries, Martin Kuster, Naziema Joeman, and Eric Schreurs (Lab. of Acoust. Imaging and Sound Control, Delft Univ. of Technol., P.O. Box 5046, 2600 GA Delft, The Netherlands)

Since it has become clear that simulation of the acoustical properties of a room is not successful unless also nonspecular reflections are taken into account, the determination of diffusion and scattering coefficients of boundary profiles is a hot topic in room acoustics research. Measurement of the impulse response of a boundary along a planar array of microphone positions enables us to calculate these coefficients in situ, avoiding the drawbacks of laboratory measurements (necessity of scale modeling, limited value of results for practical situations). Array technology makes it also possible to extrapolate the measured responses to the boundary surface, thus creating an acoustical image of the boundary. Next, scattering objects can be virtually added to this image and the impulse response of the modified configuration at the array position can be obtained by inverse extrapolation. This way, the effect of adding an object to a real environment is simulated in a highly realistic way. For several (virtual) modifications of the original boundary, diffusion and scattering coefficients have been calculated and compared. Also, listening tests have been carried out to assess the perceptual significance of the modifications.

\section{1:50}

3pAAb4. Effects of specular and diffuse reflections on perceived music quality. Pattra Smitthakorn, Martin A. Gold, Gary W. Siebein, and Bertram Y. Kinzey, Jr. (School of Architecture, Univ. of Florida, 134 ARCH, PO BOX 115702, Gainesville, FL 32611-5702, pattras@ufl.edu)

The purpose of this study was to investigate the effects of temporal distribution of diffuse and specular reflections on the perceived acoustic qualities of music performance. Sets of impulse responses were designed with different temporal distributions of early acoustic energy (specular and diffuse reflections). Then, three types of anechoic sound sources, orchestral music, trumpet, and piano, were convolved with the designed impulse responses. The results from the listening tests revealed that different room environments were needed to acoustically support different source characteristics: (1) A large number of diffuse reflections arriving within 40 and $80 \mathrm{~ms}$ of the direct sound improved perceived intimacy, texture, and overall impression for all sound sources; heightened perceived clarity for trumpet and piano; and reduced perceived glare for trumpet. (2) Diffuse reflections arriving between 80 and $160 \mathrm{~ms}$ of the direct sound preserved perceived reverberance and reduced perceived echoes as opposed to specular reflections arriving at the same time period. The results of this study indicate that music performance halls should be designed to include diffuse reflections from surfaces within the 80 -ms time period to achieve preferred texture, intimacy, clarity, and overall impression and in the 160-ms time period to reduce echoes.

\section{2:05}

3pAAb5. The angular distribution of sound incident on a panel or opening. John L. Davy and Thomas K. John (RMIT Univ., GPO Box 2476V, Melbourne, Victoria 3001, Australia, john.davy@ @rmit.edu.au)

A theoretical method has been developed for predicting the directivity of the sound that is radiated from a panel or opening excited by sound incident on the other side. This directivity needs to be known when predicting the sound level at a particular position due to sound radiation from a roof, wall, ventilating duct, or chimney flue. The method uses a twodimensional strip model and the low-frequency result for a square piston. It was found necessary to use a weighting function in order to account for the angular distribution of the incident sound. Initially a cosine squared weighting function with a weighting angle parameter was chosen and the weighting angle parameter was varied in order to obtain the best agreement with experiment for the particular situation. This talk will describe the theoretical development of weighting functions, which are based on the actual physics of each situation. Situations that are covered include an opening or panel on the surface of a room and an opening at the end of a duct. The method will be compared with published experimental results on the directivity of the forced sound radiation from panels and the sound radiation from openings.

\section{2:20}

3pAAb6. Spatial coverage of reflector panels predicted with and without edge diffraction. Jonathan Rathsam, Lily $M$. Wang (Architectural Eng. Program, Univ. of Nebraska-Lincoln, 247 PKI, 1110 S. 67th St., Omaha, NE 68182-0681), and Rendell R. Torres (Rensselaer Polytechnic Inst., Troy, NY 12180-3590)

This investigation concerns the spatial coverage of reflector panels. The specular reflection zone (SRZ) is the spatial coverage predicted by geometrical acoustics, which is commonly used when designing reflector panels. A more accurate prediction of spatial coverage accounting for edge diffraction from the panels is called the effective scattered zone (ESZ). At higher frequencies, the SRZ and the ESZ are typically similar, but notable differences emerge at lower frequencies. In this study a variety of reflector panel arrays will be examined using a boundary element method (BEM) model based on the Kirchhoff-Helmholtz integral equation. The effective scattered zone is studied as a function of incidence angle, distance from array to source and receiver, and $k a$, the ratio of panel dimension to wavelength. The authors seek rules of thumb to predict configurations in which the SRZ and ESZ differ most notably. [Work supported by National Science Foundation.]

\section{2:35}

3pAAb7. Variable reflection acoustic wall system by active sound radiation. Genta Yamauchi and Akira Omoto (Omoto Lab., Dept. of Acoust. Design, Faculty of Design, Kyushu Univ., Shiobaru 4-9-1, Minami, Fukuoka 815-8540, Japan)

For acoustic design of special spaces such as studios, it might be necessary to realize a diffuse reflection from the specific wall surface. Moreover, this requirement often changes according to the time or situation. In this study, a new acoustic wall system is examined; it is a variable reflection acoustic wall system that can address such strict requirements. This system fundamentally comprises a loudspeaker, a pick-up microphone, and absorbent material. Varying the amplitude of the radiation sound that is picked up by the microphone and reradiated by the loudspeaker mounted behind the absorbing material therefore corresponds to varying the reflection performance of the wall system. Several models are proposed. The measurement results show that the proposed models can realize various absorption characteristics. Additionally, these models can realize various radiation characteristics: diffuse reflection and inclined reflection to a desired direction. Furthermore, the frequency characteristic of simulated radiation is changed, so the proposed system can generate reflection coefficient with intended frequency characteristics. The design of an effective feedback canceller between the pick-up microphones is the subject of ongoing research toward practical use of this proposed system. 
3pAAb8. Helmholtz resonator array for low-frequency sound absorption. SangRyul Kim (Acoust. Lab., Korea Inst. of Machinery and Mater., 171 Jang-dong, Yuseong-gu, Daejeon, 305-343, Korea, srkim@kimm.re.kr) and Yang-Hann Kim (Korea Adv. Inst. of Sci. and Technol., Sci. Town, Daejeon, 305-701, Korea)

An array of Helmholtz resonators is often used for reducing lowfrequency noise because of the high performance at its resonance frequency. One of the very attractive characteristics of the resonator array is that its effective frequency band is much wider than what can be obtained by a single resonator. This paper discusses a method of designing a Helmholtz resonator array panel for low-frequency sound absorption. First, various experimental results are introduced and studied. Second, based on the experimental results, a theoretical method is presented that can predict reasonably well the panel's absorption characteristics. Any configuration of resonator arrangement can be treated in the method, while all the resonators may or may not be identical. Comparisons between the predicted and experimental results reveal the accuracy of the proposed method. Finally, a numerical optimization is performed to design the Helmholtz resonator array panel. Some examples of optimal designs under some restrictions are also introduced.
3pAAb9. Concept of active reverberation box. Hisaharu Suzuki, Akira Omoto, and Kyoji Fujiwara (Kyushu Univ., Shiobaru 4-9-1, Minamiku, Fukuoka 815-8540, Japan)

A concept of a small reverberation box is proposed. This box has an active controller that can control the energy flow direction: it is a controller for the sound intensity. Consequently, the box is useful to measure the absorption characteristics of materials, even in a low-frequency range. The controller can create an arbitrary incident condition towards the materials. The measurement procedure with this "active reverberation box" is assumed as follows: (1) The incident angle of the sound is changed by the controller. (2) Impulse responses are measured in some points. (3) Steps 1 and 2 are repeated many times and impulse responses are averaged in an energy domain. (4) The reverberation time is calculated using the averaged impulse response. (5) Absorption characteristics are calculated by comparing the reverberation time of the empty room. The controller is formulized using a pseudo-Hermitian quadratic form of the sound intensity in the box. Numerical examination is performed using the finite difference time domain (FDTD) method. Experimental results demonstrate the effectiveness of the controller's performance. Availability of the active reverberation box is examined using some conventional measurement methods. Numerical results correspond approximately to the absorption coefficients that were taken in a reverberation chamber.

\title{
Session 3pAB
}

\section{Animal Bioacoustics: Vertebrate Vocal Production: Themes and Variations}

\author{
W. Tecumseh Fitch, Cochair \\ Univ. of St. Andrews, School of Psychology, Fife KY16 9JP, U.K. \\ Kiyoshi Honda, Cochair \\ ATR Human Information and Processing Labs., 2-2 Hikaridai, Seika-Cho, Soraku-Gun, Kyoto 619-0288, Japan
}

Chair's Introduction-1:00

Invited Papers

1:05

3pAB1. Vertebrate vocal production: Themes and variations. W. Tecumseh Fitch (Univ. of St. Andrews, St. Andrews, Fife KY169JP, UK)

Most terrestrial vertebrates produce sound using a vocal system of lungs, larynx, and vocal tract, already in place in lungfish ancestral to all tetrapods. Despite the conservative nature of this basic system, there are a wide variety of modifications of the structure of the vocal system that appear to be linked to vocalization. In addition, several clades have evolved novel vocal production systems, including the avian syrinx and the odontocete nasal sac system. In this overview I will focus on the "standard" vertebrate system, discussing modifications of the vocal folds to produce high-pitched (e.g., bats and some primates) or low pitched (e.g., roaring cats) vocalizations, showing endoscopic videos of such unusual vocal folds in action. Moving to the vocal tract, a number of anatomical modifications result in vocal tract elongation, thus lowering formant frequencies. The flexible, dynamic nature of the mammalian vocal tract is illustrated with cineradiographs of vocalizing mammals. Finally, I will discuss the enigmatic existence of air sacs attached to the larynx or vocal tract. Vocal air sacs have evolved in parallel in many different species, in all classes. Despite a variety of plausible hypotheses, the function(s) of these air sacs remains poorly understood. 
3pAB2. Simulation of Siberian tiger vocalization. Ingo R. Titze, Fariborz Alipour (The Univ. of Iowa, Iowa City, IA 52242; Natl. Ctr. for Voice and Speech, The Denver Ctr. for the Perfoming Arts, Denver, CO 80204), Wm. Tecumseh Fitch (Univ. of St. Andrews, St. Andrews, Fife, UK), Eric J. Hunter (The Denver Ctr. for the Performing Arts, Denver, CO 80204), and Edward J. Walsh (Creighton Univ., Omaha, NE)

The excised larynx of a Siberian tiger, which died of natural causes, was studied on a laboratory bench. Pressure-flow relations were determined, as well as modes of vibration of the vocal pads. In addition, CT scans of the animal were made available (from previous health examinations), which allowed the vocal-tract airway to be quantified. The larynx and vocal tract-data were then used to simulate the sound production of the animal. Results showed that multiple tissue modes are excited to produce a tiger roar, and that the production is highly efficient for aerodynamic to acoustic energy conversion. Scaling factors for linear dimensions of the larynx and vocal tract are derived to compare the phonation of this large mammal to human phonation.

\section{$1: 45$}

3pAB3. Nonlinear phenomena in vertebrate vocal production. Hans-Peter Herzel, Riccardo Zaccarelli, Tobias Riede (Inst. for Theoretical Biol., Invalidenstr. 43, 10115 Berlin, Germany), Juergen Neubauer (UCLA, Los Angeles, CA 90095-1794), and W. Tecumseh FitchIII (Univ. of St. Andrews, KY16 9JU, Scotland, UK)

Nonlinear phenomena such as subharmonics, biphonation (two independent frequencies), and deterministic chaos are widespread in animal communication. Examples from primate vocalizations, African wild dogs, hyena whoops, and bird songs are presented. Despite the variability of size and morphology, vocal production in vertebrates is governed by the same underlying mechanism: vibrating tissues such as vocal folds, membranes, or labia are driven by overcritical airflow. Consequently, sophisticated biomechanical-aerodynamic models of human voice production can serve as a starting point for modeling nonhuman vocal production. However, there is a variety of additional anatomical features in diverse species: pronounced air sacs, extensions of the vocal folds termed "vocal membranes" or "vocal lips," or the coordinated action of left and right syrinx in song birds. It is shown that such specific features can induce voice instabilities. For instance, source-tract interactions lead to subharmonics and chaos, and vocal membranes allow multiple "registers." Finally bifurcations in biomechanical models of the syrinx are discussed. [Work supported by the Deutsche Forschungsgemeinschaft (Grant He 2168/7).]

2:05

3pAB4. Mosaic and multiple-step evolution of the anatomical foundations for human speech. Takeshi Nishimura (Dept. of Zoology, Kyoto Univ., Kitashirakawa Oiwake-cho, Sakyo, 606-8506 Kyoto, Japan, nishimur@anthro.zool.kyoto-u.ac.jp)

The human vocal tract develops to form a unique configuration with equally long horizontal and vertical cavities. This anatomy contributes greatly to the morphological foundations of human speech. It depends on the reduced growth of the facial length and on the major descent of the larynx relative to the palate. Anatomically, the laryngeal descent is accomplished through both the descent of the laryngeal skeleton relative to the hyoid and the descent of the hyoid relative to the palate. Here, the recent progresses are surveyed in the studies on the developments of the vocal tract anatomy in humans, chimpanzees, and macaques. They showed that humans and chimpanzees fully share the major descent of the larynx with the epiglottis descent. Macaques also show the hyoid descent, but this is not accompanied by the descent of the laryngeal skeleton and epiglottis. It is argued that the major descent of the larynx has probably evolved at least in two steps before the divergence of the human from the chimpanzee lineage, originally to confer an advantage via a function unrelated to speech. Thus, facial flattening has probably evolved only secondarily with anatomical consequences that facilitated speech in the human lineage. [Work supported by JSPS Grant-in-Aid (No. 16000326).]

$2: 25$

3pAB5. Birdsong: The role of the vocal tract. Roderick A. Suthers (School of Medicine and Dept. of Biol., Jordan Hall, Indiana Univ., Bloomington, IN 47405, suthers@indiana.edu)

The avian vocal organ, the syrinx, is unique among vertebrates in its morphology and location at or near the base of the trachea. In oscine songbirds, two independent sound sources share the same vocal tract. Despite a growing knowledge of the neural and syringeal mechanisms underlying song, the acoustic role of the suprasyringeal vocal tract in modulating birdsong is still poorly understood. During song, the beak opening, or gape, is typically positively correlated with the dominant frequency of the vocalization, but the mechanism by which beak gape influences vocal tract acoustics is controversial. X-ray cinematography of singing birds reveals song-related movements of the hyoid apparatus and larynx that cause the volume of the upper vocal tract to vary inversely with the songs fundamental frequency. At low fundamental frequencies, the oropharynx and cranial end of the esophagus expand to form a single large chamber, the volume of which is reduced at higher frequencies by collapse of the esophagus and reduction of the oropharyngeal cavity. A computational acoustic model indicates that birds actively tune the volume of their oropharyngeal-esophageal cavity so that its major resonance tracks the fundamental frequency of the song. [Work supported by NIH.]

\section{$2: 45$}

3pAB6. Odontocete nasalizations: Morphology, physiology, and evolution of sonar signal generation in toothed whales. Ted Cranford (Biol. Dept., San Diego State Univ., 5500 Campanile Dr., San Diego, CA 92182-0001)

The odontocete nasal apparatus contains tissue complexes that generate a variety of sounds. Sounds are produced by moving air across the phonic lips, internal structures that were formed by the invagination of the ancient nasal openings. X-ray computed tomography shows this sound generation complex bilaterally in all odontocetes except in sperm whales, where it is unilateral. Two bottlenose dolphins provided simultaneous bilateral recordings of internal nasal cavity pressure and nasal tissue motion. Small catheters measured pneumatic pressure changes within each bony nasal passage; high-speed video endoscopy recorded motion at both 
pairs of phonic lips; and two hydrophones revealed acoustic pressure during target discrimination. Sonar pulses can be generated at the left and right phonic lips, independently or simultaneously. Air pressure in both bony nasal passages rises and falls together, even if the activity patterns at the two pairs of lips are different. Increasing pulse repetition rate or sound pressure levels are all normally accompanied by increasing nasal air pressure. The bilateral arrangement of sonar sources in the odontocete nose provides a basis for reinterpretation of previous findings; reevaluation of physiologic limits and on pulse repetition rate, signal bandwidth, frequency composition, and transmission beam geometry; and the evolution of this system.

\section{Contributed Papers}

3:05

3pAB7. The creative walrus: Novel sound production via contingency learning. Ronald J. Schusterman, Colleen Reichmuth Kastak (Inst. of Marine Sci., Long Marine Lab., Univ. of California, Santa Cruz, 100 Shaffer Rd., Santa Cruz, CA 95060), Debbie Quihuis (Six Flags Marine World, Vallejo, CA 94589), and Marla M. Holt (Univ. of California, Santa Cruz, Santa Cruz, CA, 95060)

Walruses (Odobenus rosmarus) have a range of anatomical specializations that can provide plasticity to their vocal emissions. These include respiratory adaptations related to breath-holding and buoyancy and soft tissue adaptations of the mouth, lips, and tongue related to suction feeding. The aim of this study was to examine the extent to which contingency learning using food reinforcement could modify the sound production of walruses. One male and three female captive walruses participated in the current study. Animals were first trained in air in a series of four experimental steps: (1) induce and reinforce natural sound production, (2) establish stimulus control over sound production and inhibition, (3) establish stimulus control over different sound types by mapping them to different discriminative stimuli, and (4) encourage the production of novel sounds by withholding reinforcement for previously emitted sounds and reinforcing only novel variants. Subsequently, two of the walruses were transferred from airborne to underwater testing. In the absence of additional training, both individuals, when prompted to vocalize, emitted a range of underwater sounds, many of which were qualitatively different from those produced in air and those recorded in baseline conditions under water. Thus, walruses, like cetaceans, can learn to modify their sound production.

\section{3:20-3:35 Break}

\section{$3: 35$}

3pAB8. Acoustical development of the human baby cry: Anatomical and social factors. Yulri Nonaka, Noriko Kudo, Kazuo Okanoya (Lab. for Biolinguistics, RIKEN Brain Sci. Insitute, 2-1 Hirosawa, Wako, Saitama 351-0198, Japan, ynonaka@brain.riken.jp), Noriko Kudo, Kazuo Okanoya (Chiba Univ.1-33 Yayoi-Cho, Inage, Chiba 263-8522, Japan), Noriko Kudo (JSPS), and Kazuo Okanoya (PRESTO, JST)

We investigated the acoustical development of the human baby cry based on our two-stage hypothesis, which holds that two distinct factors affect the development of the baby cry. First, humans possess the corticobulbar pathway connecting the face motor cortex and the medullary respiratory and vocal centers. This pathway is absent in nonhuman primates and other mammals. We postulate that the myelinization of this pathway would change the acoustic characteristics of the baby cry. Second, after neuroanatomical maturation enabled variable cry patterns, the social relationships with caregivers should differentiate the acoustical patterns of the baby cry. This should lead to family-specific cry patterns. To test the two-stage hypothesis, we recorded ten newborn babies until they were 1 year old. In all babies, the cry pattern was very simple up to 2 weeks, while after 1 month, the pattern became temporally and spectrally complex, suggesting neuroanatomical changes. At three months, we asked the mothers to classify the cries of their own babies into three categories: sleepy, hungry, and wet diaper. There were no acoustic patterns common to their classifications, suggesting that baby cry semantics are familyspecific. These results support our two-stage hypothesis of cry development. [Work supported by JST and JSPS.]
$3: 50$

3pAB9. Is the squirrel monkey larynx lateralized biomechanically? Charles H. Brown (Dept. of Psych., Univ. of South Alabama, Mobile, AL 36688, cbrown@usouthal.edu) and Fariborz Alipour (Univ. of Iowa, Iowa City, IA 52242)

Squirrel monkey larynges were dissected, mounted on a pseudotracheal tube, and phonated via the flow of compressed heated and humidified air. Synchronized audio and video signals were digitized and analyzed with a signal processing workstation. Image analysis algorithms measured the change in glottal area due to the movement of the left and right vocal folds, respectively, and measured the change in location of both vocal folds during phonation. The results show that the squirrel monkey larynx is capable of both unilateral and bilateral oscillation. In the unilateral case, oscillation is virtually confined to the left vocal fold. The converse of this has not been observed. In the case of bilateral oscillation, the folds may oscillate synchronously in phase, asynchronously out of phase, or at different fundamental frequencies. The results are consistent with the idea that the biomechanical properties of the left and right vocal folds differ with the left fold capable of oscillation at lower subglottal pressures and greater amplitudes. Changes in vocal fold elongation and adduction change the coupling between the two folds and produce different regimes of oscillation. Squirrel monkeys appear to employ intentional shifts between different regimes of phonation to expand the size of the vocal repertoire.

\section{4:05}

3pAB10. Measuring emotional arousal in the voiced sounds of two mammals, the rhesus monkey and African elephant. Joseph Soltis, Christina Wesolek, Anne Savage (Education and Sci., Disney's Animal Kingdom, Lake Buena Vista, FL), Kirsten Leong (Cornell Univ., Ithaca, NY), and John Newman (NICHD, Poolesville, MD)

Emotional arousal is expressed in the voiced sounds of primates and other mammals, but there are no consistent acoustic measures used and few comparative analyses. We apply a representative suite of source and filter measurements to rhesus macaque (Macaca mulatta) coo calls on Cayo Santiago, Puerto Rico, and African elephant (Loxodonta africana) rumble calls at Disney's Animal Kingdom, Florida, U.S.A. Based on social context, calls were classified into high and low arousal categories. In both species, MANOVA showed that the 15 measures taken together separated calls across arousal categories. In rhesus macaques, high arousal was associated with increased and more variable fundamental frequencies, increased amplitudes in the lower frequencies, and a shift in formant locations. In African elephants, increased arousal was associated with a shift of energy from lower to higher frequencies. In addition, low ranking females expressed a greater magnitude of acoustic change compared to high ranking females. The suite of acoustic features used here may successfully characterize arousal state in a variety of mammals, but the specific acoustic features that reflect arousal and the specific pattern of acoustic response may vary by species and individual. [Work supported by Grant Nos. NIH CM-5-P40RR003640-13 and NSF-IIS-0326395.]

\section{$4: 20$}

3pAB11. Antiphonal exchanges in female African elephants. Katherine Leighty, Joseph Soltis, Anne Savage (Education and Sci., Disney's Animal Kingdom, Lake Buena Vista, FL), and Kirsten Leong (Cornell Univ., Ithaca, NY)

Female African elephants (Loxodonta africana) engage in antiphonal exchanges of rumble vocalizations. In this study, female African elephants $(N=7)$ housed at Disney's Animal Kingdom were outfitted with audiorecording collars and videotaped during 50 1-hour observation sessions 
(conducted in 2002). We found that production of the antiphonal response rumble reflected changes in herd affiliation that had taken place since our previous study (2000). Second, we examined response latency and found that females responded more quickly to the rumbles of affiliated partners than to those of a nonaffiliated female. Since affiliated partners spend more time in close proximity by definition, they may engage in higher rates of temporally associated calling simply because nearby stimuli impact them simultaneously or due to a social facilitation effect, not because they are true vocal exchanges. Therefore, we examined antiphonal rumble responses when female pairs were more than $25 \mathrm{~m}$ apart. We found that at these distances, affiliated females still exchanged rumbles more frequently, and responded at a decreased latency, than nonaffiliated pairs. Our results provide support for functional hypotheses of African elephant rumble vocalizations that require instances of temporally associated calling in order to be true communicative events. [Work supported by Grant No. NSF-IIS0326395.]

\section{4:35}

3pAB12. Commonality in the vocalizations of order Perissodactyla. David G. Browning (Dept. of Phys., Univ. of Rhode Island, Kingston, RI 02881, decibeldb@aol.com) and Peter M. Scheifele (Univ. of Connecticut, Storrs, CT 06269)

As a follow-on to the human genome project, it has been suggested that the next major gene sequencing program may be for the horse as a representative of the relatively small order Perrissodactyla. This order consists of three families; Equidae (equines), Tapiridae (tapirs), and Rhinocerotidae (rhinos), for a total of 17 species. Both sensual ability and environmental conditions (open plains to dense jungles) vary greatly among this small group; for example, horses have excellent eyesight, tapirs do not. Vocalizations of the various species are compared to determine any common characteristics that might serve as a basis for future association with specific genes. The universal quality appears to be the ability to vary pitch during the vocalization. Other subsets such as braying or whinnying exist among a limited number of species, which suggests that there may be evolutionary trends within the order.

\section{4:50}

3pAB13. Application of the source-filter theory to the study of loud-calling in male deer. David Reby (Dept. of Psych., Univ. of Sussex, Brighton BN2 9YU, UK)

I will review recent studies of roaring in red deer males, which illustrate how source-filter theory can be successfully applied to studying mammal vocal signals. Combining anatomical analyses of the vocal apparatus, acoustical analyses, and playback experiments using resynthesized calls, we have shown that $(i)$ red deer and fallow deer males have a descended and mobile larynx, an anatomical innovation that was previously believed to be uniquely human and that enables callers to modulate their formants during vocalising; (ii) minimum formant frequencies provide an honest indication of body size in red deer roars, (iii) stags use rivals' minimum formant frequencies in assessment during male-male contests, and adjust the formants of their own replies in relation to what they hear; and (iv) hinds perceive size-related variation in formant frequencies, and pay more attention to roars in which formant spacing indicates larger males, independently of fundamental frequency. I will then compare the acoustic structure of sexual loud calls in related subspecies (Corsican deer, Wapiti) and species (fallow deer, pere Davids deer), highlighting the variability of source- and filter-related acoustic features in this group. To conclude I will suggest directions for future research in this area.

\section{5:05}

3pAB14. Measuring amplitude in North American bison bellows: Environmental, social, and individual impacts. Megan Wyman, Lynette Hart, Brenda McCowan (Univ. of California-Davis, 1025 Haring Hall, One Shields Ave., Davis, CA 95616, mtwyman@ucdavis.edu), and Mike Mooring (Point Loma Nazarene Univ., San Diego, CA 92106)

Communication is a key aspect of any mating system and is a dramatic feature in the mating behavior of North American bison. During their reproductive season, bison bulls defend females from other males by establishing dominance through contests involving multiple signals, the most prominent being the bellow vocalization. While acknowledged that amplitude may be involved in sexual selection, it is rarely measured directly in the field (especially in large mammals) due to difficulties in accurately assessing this parameter. Using strict methodologies accounting for many sources of potential error, the impact that a variety of factors had on bellow amplitude was examined. Amplitude was measured in $\mathrm{dB}$ with $L_{a_{\max }}$ and $L_{c_{\text {peak }}}$ weighting. Parameters increasing amplitude (order of decreasing impact): Number of surrounding bulls bellowing, wind levels, and number of surrounding bulls. Parameters decreasing amplitude (order of decreasing impact): Distance, head orientation, days spent in rut, time of day, number of copulations, dominance level of bull, and temperature. Since amplitude is correlated with measures of bull quality, condition, and surrounding social environments (beyond physical impacts of distance, etc.), it is likely that amplitude plays a significant role in competition over mates. [Work supported by NSF.]

\section{$5: 20$}

3pAB15. Syllable chunking in Bengalese finches in a multi-tutor environment. Miki Takahasi, Iori Tomizawa, and Kazuo Okanoya (Lab. for Biolinguistics, RIKEN Brain Sci. Inst., 2-1 Hirosawa, Wako, Saitama, 351-0198 Japan, mtakahas@brain.riken.jp)

Experiments in birdsong learning have traditionally involved one tutor, usually the father, and the proportion and accuracy of note copying are discussed as indexes of song learning. But in the wild, there are several conspecific males around song-learning pupils. To test song learning under naturalistic situation, 11 male and ten female Bengalese finches were kept in a large flight cage containing ten pot-shaped nests. Seventy-eight chicks were born in this aviary over an 18-month period. We recorded the full songs of male birds after they had matured, and compared the songs between offspring and the original adult males. Most of them learned their songs from one or two tutors and some learned from three or four tutors. When a bird learned from multiple tutors, each of these tutor songs was copied in chunks and within a chunk the order of the song elements was the same as that observed in the tutor male. Furthermore, Bengalese finches change the order of chunks as they sing, and it was possible to express the rule using a finite-state syntax. Therefore, Bengalese finches have an innate universal grammar allowing them to construct a finite-state syntax from multiple sources. [Work supported by JSPS and JST.] 


\title{
Session 3pBB
}

\section{Biomedical Ultrasound/Bioresponse to Vibration and Physical Acoustics: Celebration of Floyd Dunn II}

\author{
Michael L. Oelze, Cochair \\ Univ. of Illinois, Electrical and Computer Engineering, Beckman Inst., 405 N. Mathews, Urbana, IL 61801 \\ Shin-ichiro Umemura, Cochair \\ Kyoto Univ., School of Health Science, Kyoto 607-8507 Japan
}

Chair's Introduction-1:00

Invited Papers

1:05

\begin{abstract}
3pBB1. A study on ultrasound parameter specification by temperature rise. Hiroshi Inoue and Chiaki Yamaya (Dept. of Electron. and Elec. Eng., Akita Univ., 1-1 Tegatagakuen-cho Akita-shi, Akita, 010-8502 Japan, inoueh@ gipc.akita-u.ac.jp)

So far, temperature rises by absorption of ultrasound have been researched to secure the safety of the ultrasonic diagnostics and the application of ultrasonic therapy. This research is intended to develop a numerical simulation method that is applicable to estimation of medium constants, e.g., the absorption coefficient, using the temperature rise by the ultrasound. The temperature rises have been measured using a micro-thermocouple with high spatial resolution. Three-dimensional simulation using finite-difference time-domain (FDTD) method, which combines ultrasonic propagation and heat conduction, i.e., ultrasonic and temperature fields, is developed. At the first stage, two-dimensional analyses of ultrasonic fields were different from theoretical and experimental values. Therefore, we developed three-dimensional analysis. Time responses and spatial distributions of temperatures of the experiments and simulations were compared. Spatial distributions of temperature that were obtained using the FDTD simulation correspond qualitatively with those obtained by experiment, taking into account the thermocouple artifact. The possibility of estimation of medium constants is therefore suggested.
\end{abstract}

$1: 25$

3pBB2. Modern acoustic microscopy and applications. Lawrence Kessler (Sonoscan, Inc., 2149 E. Pratt, Elk Grove Village, IL 60007)

The concept of acoustic microscopy was first suggested in the late 1930s by S. A. Sokolov and first demonstrated in a onedimensional scan by Floyd Dunn and W. J. Fry in 1959. The notion of producing images of materials and biological tissues with UHF ultrasound to characterize their microelastic properties has broad appeal, however, most of the modern day (commercial) systems have been used instead for defect detection in manufactured products such as semiconductor devices. This paper will review a spectrum of applications from both the materials and biological worlds.

\section{$1: 45$}

3pBB3. Twenty years' development of acoustic microscopy for medicine and biology. Yoshifumi Saijo, Hidehiko Sasaki, Motonao Tanaka (Dept of Medical Eng. \& Cardiology, Tohoku Univ, 4-1 Seiryomachi, Aoba-ku, Sendai 980-8575, Japan, saijo@idac.tohoku.ac.jp), Naohiro Hozumi (Aichi Inst. of Technol., Yakusa-cho, Toyota 470-0392, Japan), Kazuto Kobayashi (Honda Electron. Co. Ltd., Oiwacho, Toyohashi 441-3193, Japan), and Floyd Dunn (Univ. of Illinois, Urbana-Champaign, IL)

Since 1985, scanning acoustic microscope (SAM) systems for medicine and biology has been developed and have been equipped for investigation of acoustic properties of various organs at Tohoku University. There are three objectives of SAM for biomedicine. First, it is useful for intraoperative pathological examination because staining is not required. Second, it helps understanding lower frequency ultrasonic images such as echography or intravascular ultrasound because SAM provides microscopic acoustic properties. Third, it provides information on biomechanics of tissues and cells. The most important feature of our SAM is to measure attenuation and sound speed of soft tissues by analyzing the frequency-dependent characteristics of amplitude and phase. Recently, a new-concept acoustic microscopy using a single pulsed wave instead of burst waves used in conventional SAM systems was proposed. Application of SAM has also been broadened and it covers not only myocardium and atherosclerosis in cardiology but also skin, bone, cartilage, tendon, and muscle in dermatology and orthopedic surgery. Higher resolution acoustic microscopy with over $500 \mathrm{MHz}$ enabled us to visualize cultured cells and it can be applied for quality assessment of regenerated skins and cartilage. Impedance acoustic microscopy and 3-D acoustic microscopy are developed for in vivo tissue imaging. 
3pBB4. VHF/UHF range bioultrasonic spectroscopy system and method. Naoyuki Akashi (Ichinoseki Natl. College of Technol., Ichinoseki 021-8511, Japan, akashi@ichinoseki.ac.jp), Mototaka Arakawa, Hiroyuki Odagawa, Toshio Sannomiya, Jun-ichi Kushibiki (Tohoku Univ., Sendai 980-8579, Japan), Noriyoshi Chubachi (Tohoku Univ., Sendai 980-8579, Japan), and Floyd Dunn (Univ. of Illinois, Urbana, IL 61801)

We developed the bioultrasonic spectroscopy system and method for doing ultrasonic tissue characterization studies in the VHF and UHF ranges. The system can determine the frequency characteristics of bulk acoustic properties of the sound velocity, attenuation, acoustic impedance, and density simultaneously by an ultrasonic transmission line comparison method using pure water as the reference, and using rf tone burst pulse signals. A biological tissue/liquid specimen is sandwiched between the parallel surfaces of buffer rods having $\mathrm{ZnO}$ piezoelectric film transducers on their opposite ends. Measurements are conducted with a typical thickness of 0.1 to $1 \mathrm{~mm}$ in the frequency range up to $700 \mathrm{MHz}$, and in the frequencies higher than $500 \mathrm{MHz}$, with a thin layer of specimen, several micrometers, operating in the multiple interference method. The system was applied for demonstration to measure the acoustic properties of bovine tissues, egg yolk and albumen, and aqueous solutions of biomacromolecules such as bovine hemoglobin and sugars in the frequency range $10 \mathrm{MHz}$ to $1.6 \mathrm{GHz}$. The system and methodology will enable us to provide basic data for understanding the interaction of high-frequency ultrasonic waves with biological media and to make contributions to the establishment of the scanning acoustic microscope in the fields of biological and medical sciences.

\section{2:25}

3pBB5. Floyd's contribution to B/A measurement of biological materials. Leon A. Frizzell (Univ. of Illinois, 405 N. Mathews Ave., Urbana, IL 61801)

Ultrasound as used for medical diagnosis and high-intensity therapy is of sufficient amplitude that the effects of nonlinear propagation are important. Critical to modeling and understanding these nonlinear effects is knowledge of the nonlinearity parameter, $\mathrm{B} / \mathrm{A}$, for the biological tissues involved. In a series of papers Floyd Dunn and colleagues determined the value of B/A for several biological materials and soft tissues using the thermodynamic and finite amplitude methods. They showed that the B/A value depended directly upon the solute concentration in protein solutions. The value of B/A was around 7 for many soft tissues, including liver, muscle, and brain, but was around 11 for fat. Homogenized liver had a value that was less than that for whole liver, demonstrating an effect of structure. These results provided information regarding the range of B/A for biological materials and its dependence on concentration and structure and suggested the possible use of B/A in tissue characterization.

3pBB6. The impact of Floyd Dunn's research on echocardiography and myocardial tissue characterization. James G. Miller (Dept. of Phys., Washington Univ., St. Louis, MO 63130, james.g.miller@wustl.edu)

By the mid-1970s our lab had demonstrated that alterations in myocardium as a result of mature infarction could be quantified ultrasonically, with increased attenuation correlating with the extent of myocardial damage assessed in the same sites by depletion of creatine kinase. [Mimbs et al., Circ. Res., 41, 192-198 (1977)]. The Feigenbaum laboratory made the qualitative observation that zones of infarct were "more echo producing" than nearby zones. [Rasmussen et al., Circulation 57, 230-237 (1978)]. A 1973 article from Floyd Dunn's laboratory [S. Fields and F. Dunn, J. Acoust. Soc. Am. 54, 809-812 (1973)] provided the basis for understanding these and many future developments in echocardiography and myocardial tissue characterization. In that seminal paper the authors state, "The magnitude of the echoes received is directly related to the preferred orientation and amount of collagen ...." We subsequently demonstrated the causal relationships between regional myocardial collagen content and both attenuation and backscatter. We also confirmed the Dunn prediction of anisotropy of attenuation, backscatter, and phase velocity. [Work supported by Grant NIH-R37-HL40302.]

3:05-3:20 Break

3pBB7. Theoretical analysis of local attenuation approximations when calculating scatterer correlation length. Timothy A. Bigelow (Univ. of North Dakota, 243 Centennial Dr., Stop 7165, Grand Forks, ND 58202) and William D. O’Brien, Jr. (Univ. of Illinois, Urbana, IL 61801)

Determining the correlation length of ultrasound scatterers has shown potential in many tissue characterization applications. Accurate estimates of scatterer correlation length can only be obtained after correcting for focusing, frequency-dependent attenuation along the propagation path (total attenuation), and frequency-dependent attenuation in the region of interest (local attenuation). In order to correct for the local and total attenuation, an approximation is required because uncertainties in tissue attenuation can easily be $0.5 \mathrm{~dB} / \mathrm{cm}-\mathrm{MHz}$, or greater, even for the same tissue type [J. Acoust. Soc. Am. 64, 423 (1978)]. Earlier simulation results demonstrated that the most robust approximation for the local attenuation while completely compensating for total attenuation and focusing for weakly focused sources was to assume a finite frequency dependence of $\sim 0.8 \mathrm{~dB} / \mathrm{cm}-\mathrm{MHz}$ independent of the true attenuation of the tissue for attenuation values between 0.05 and $1 \mathrm{~dB} / \mathrm{cm}-\mathrm{MHz}$. The goal of the present study was to investigate the theoretical basis for this approximation so that the results could be applied to other ranges of attenuation values. The theoretical analysis was then compared to the simulation results to validate the conclusions. [This work was supported by the University of Illinois Research Board and the University of North Dakota School of Engineering and Mines.] 
3pBB8. High-frequency pulse-compression ultrasound imaging using an 18-MHz annular array. Jonathan Mamou, Jeffrey A. Ketterling, and Ronald H. Silverman (F. L. Lizzi Ctr. for Biomed. Eng., Riverside Res. Inst., 156 William St., New York, NY 10038)

High-frequency ultrasound (HFU) allows fine-resolution imaging at the expense of limited depth-of-field (DOF) and shallow acoustic penetration. Coded-excitation imaging allows a significant increase in the signal-to-noise ratio (SNR) and acoustic penetration depth. A 18-MHz, five-element annular array with a focal length of $31 \mathrm{~mm}$ and a total aperture of $10 \mathrm{~mm}$ was made using a 25$\mu \mathrm{m}$ thick membrane. To excite the PVDF-TrFe transducer, an optimized 8- $\mu$ s linear chirp spanning 6 to $30 \mathrm{MHz}$ was uploaded into a waveform generator. The optimal chirp design was deduced from ex vivo experiments and computer simulations. Images of a seven-wire phantom were generated. The $25-\mu \mathrm{m}$ wires were spaced in $1-\mathrm{mm}$ axial and lateral increments. All 25 transmit/receive signal combinations were digitized and postprocessed for compression and synthetic focusing. Compression consisted of linearly filtering the signals with a mismatched filter. The mismatched filter was the time-reversed excitation chirp modulated by a DolphChebyshev window. Marked image-quality improvements were demonstrated in images of ex vivo cow eyes. Results of this study demonstrate that the DOF can be improved by a factor of 3 and SNRs, after compression, can be improved by $24 \mathrm{~dB}$.

\section{Contributed Papers}

\section{4:00}

3pBB9. Spatial distributions of acoustic parameters in high-intensity focused ultrasound fields. Vera A. Khokhlova (Ctr. for Industrial and Medical Ultrasound, APL, Univ. of Washington, 1013 NE 40th St., Seattle, WA 98105, vera@apl.washington.edu), Olga S. Bessonova (Moscow State Univ., Moscow 119992, Russia), Michael S. Canney, Michael R. Bailey, and Lawrence A. Crum (Univ. of Washington, Seattle, WA 98105)

Different peak and average acoustic parameters determine the efficiency of different physical mechanisms of high-intensity focused ultrasound (HIFU) interaction with biological tissue. Spatial distributions of these parameters are therefore important for transducer calibration and extrapolation of measurements in water to application in tissue. In the case of linear focusing, all parameters of the acoustic field can be obtained from the spatial distribution of the wave amplitude. However, in nonlinear focused beams, each parameter has its own characteristic spatial structure, which changes with the increase of the HIFU power level. This work compares the focal size and location calculated for the peak positive and peak negative pressure, mean intensity, and effective acoustic energy absorption in water and in tissue. Numerical solutions, obtained with the KZK-type model, are analyzed for various regimes of linear, quasilinear, and strongly nonlinear propagation which includes formation of shocks. The results of simulations are validated by comparison with measurements performed with a fiber-optic probe hydrophone in water and in a tissuemimicking phantom. The peak positive pressure and effective absorption are finely focused, whereas the negative pressure, responsible for cavitation, is broad and displaced towards the transducer. [Work supported by NSBRI SMS00402, RFBR, and NIH DK43881.]

\section{4:15}

3pBB10. Finite aperiodic model of human lung. Mark S. Wochner, Yurii A. Ilinskii, Mark F. Hamilton, and Evgenia A. Zabolotskaya (Appl. Res. Labs., Univ. of Texas, P.O. Box 8029, Austin, TX 78713-8029, mwochner@arlut.utexas.edu)

This work is focused on studying the dynamics of the human lung in response to low-frequency sound. The model developed uses individual alveoli as the basic unit cells, which are represented as truncated octahedra [Y. C. Fung, J. Appl. Physiol. 64, 2132 (1988)]. Having 14 faces, this polyhedron possesses the smallest surface-to-volume ratio of any polyhedron capable of filling three-dimensional space. Unlike previous quasistatic models of this type, typically used to predict elastic properties of the lung, ours is a dynamical model. Mass is concentrated at the vertices of the polyhedra. Viscoelastic properties of the collagen and elastin in lung tissue are taken into account using a Kelvin model that is augmented to account for nonlinear elasticity. The Kelvin elements connect the point masses along the edges and across the faces of the polyhedra. Although not yet taken into account, the model permits inclusion of alveolar ducts by opening or closing selected faces of the polyhedra to accommodate airflow. Numerical simulations of small alveolar clusters subjected to different excitations and geometric constraints will be shown. [Work supported by ONR and ARL IR\&D.]
$4: 30$

3pBB11. Infinite periodic model of human lung. Yurii A. Ilinskii, Mark S. Wochner, Mark F. Hamilton, and Evgenia A. Zabolotskaya (Appl. Res. Labs., Univ. of Texas, P.O. Box 8029, Austin, TX 78713-8029)

As described in a companion abstract, an investigation is underway to develop a bioacoustic model of human lung. In parallel with the numerical model described in the companion abstract, a semianalytic model is being developed. The basic unit cell, a truncated octahedron, is the same. In contrast here, the medium is assumed to be infinite in extent. The resulting lattice possesses cubic symmetry, and, for homogeneous deformation, all material properties are determined by a single unit cell. Deformation of the unit cell is determined by the 24 vertices of the polyhedron, only 6 of which are independent. In this presentation we discuss quasistatic deformation of the discretized medium, and thus ignore inertia and energy dissipation. An analytic model for the nonlinear elasticity of collagen and elastin is used to determine the stiffnesses of the springs connecting vertices of the polyhedra. Minimization of the potential energy for a given macroscopic deformation permits calculation of microscopic deformation within the unit cell, which in turn determines the stresses and therefore the elastic constants. Sample calculations will be presented, including the longitudinal and transverse wave speeds in the medium as functions of direction and orientation. [Work supported by ONR and ARL IR\&D.]

$4: 45$

3pBB12. Detecting spatially resolved inertial cavitation with a diagnostic ultrasound system. Caleb H. Farny, Robin O. Cleveland, R. Glynn Holt, and Ronald A. Roy (Dept. of Aerosp. and Mech. Eng., Boston Univ., 110 Cummington St., Boston MA 02215, cfarny@bu.edu)

The onset and presence of inertial cavitation in high-intensity focused ultrasound (HIFU) therapy has been identified as an important indicator of energy deposition for therapy planning. A common technique for detecting inertial cavitation is passive cavitation detection, where broadband noise emissions indicative of inertially driven bubbles collapses are detected using a fixed focus transducer. This technique is suboptimal for clinical applications, because most passive cavitation detector (PCD) transducers are tightly focused and thus afford limited spatial coverage of the HIFU focal region. Here, we report on efforts to use a diagnostic ultrasound system as a PCD by operating in passive mode and filtering out the main HIFU frequencies. This approach allows for spatially resolved detection of inertial cavitation throughout the focal region while being insensitive to scattering from voids or bubbles. [Work supported by the Dept. of the Army and the Center for Subsurface Sensing and Imaging Systems.] 
3pBB13. Classification of flow-limiting thrombus in acute coronary syndrome by acoustic microscopy. Hidehiko Sasaki, Yoshifumi Saijo, Takayuki Kanno, and Motonao Tanaka (Dept of Medical Eng. \& Cardiology, Tohoku Univ, 4-1 Seiryomachi, Aoba-ku, Sendai 980-8575, Japan)

Virtual histology $(\mathrm{VH})$, based on a power spectral analysis of radiofrequency signal from intravascular ultrasound (IVUS) with neural network, can detect an unstable plaque from all the regions of coronary atherosclerosis. However, it provides limited information on plaque vulnerability because the resolution is larger than $100 \mathrm{~m}$ and thrombus is not classified precisely. The objective of the present study is to measure the acoustic properties of flow-limiting thrombus responsible for acute coronary syndrome (ACS) at the microscopic level. A newly developed acoustic microscope with $100-\mathrm{MHz}$ pulsed ultrasound was equipped to measure two-dimensional distribution of attenuation and sound speed. The tissues were obtained from coronary arteries of 26 patients with ACS by intracoronary aspiration catheter. They were sectioned at 5-mm thickness and mounted onto glass slides without cover slips or staining and used acoustic microscopy study. The values of attenuation and sound speed of the red thrombus were significantly higher than those of the platelets-rich thrombus. Both acoustic parameters of old red thrombus were significantly lower than those of the organized thrombus. The acoustic properties provided by acoustic microscopy can classify the flow-limiting thrombus at different pathological stages. The criteria will provide further information for development of a VH-IVUS algorithm.
3pBB14. Image processing for scanning-type biological ultrasonic microscope considering its beam characteristics. Kazuto Kobayashi (Honda Electron. Co., Ltd. 20 Oyamazuka, Oiwa-cho, Toyohasi, Aichi, Japan), Naohiro Hozumi (Aichi Inst. of Technol., Yakusa Toyota Aichi Japan), and Shigeo Ohtsuki (Tokyo Inst. of Technol., Midori-ku, Yokohama, Kanagawa, Japan)

We have been developing a biological ultrasonic microscope with a high lateral resolution. An acoustic pulse is transmitted by a scanning transducer to a substrate on which a thin slice of a tissue is embedded. The transducer has a minute curvature in order to focus an acoustic beam with a high frequency component. It may be fabricated by MEMS technology; however, from the point of view of engineering, it may not be easy to guarantee the reproducibility of beam pattern as specified. In a high frequency range, a small error in fabrication will lead to a big difference in beam pattern, which strongly affects the observed image. Using newly proposed methodology to observe a well-defined pin-hole, we found that each commercial single probe transducer with the same specification has a unique beam pattern. In addition, the pattern was not always concentric. Consequently, we proposed a method to compensate the image that was obtained using an eccentric beam pattern. A clearer image was successfully obtained after the compensation.

\title{
Session 3pMU
}

\author{
Musical Acoustics: Musical and Structural Aspects of Instruments \\ Nicholas J. Giordano, Cochair \\ Purdue Univ., Dept. of Physics, West Lafayette, IN 47907 \\ Isoharu Nishiguchi, Cochair \\ Kanagawa Inst. of Technology, 1030 Shimo-Ogino, Atsugi, Kanagawa 243-0292, Japan
}

\section{Contributed Papers}

\section{1:00}

3pMU1. Vibrations of a harp soundbox: Experiment and finiteelement analysis. Chris Waltham and Andrzej Kotlicki (Dept. of Phys. \& Astron., University of British Columbia, Vancouver BC, Canada V6T 1Z1, cew@phas.ubc.ca)

A medium-sized lever harp of proven design has been built. At each stage of construction, the components have been subjected to experimental modal analysis, using an impact hammer and small accelerometer, and also Chladni patterns. The results have been compared to a corresponding sequence of finite-element calculations. The aim is to build a picture of how the various ingredients-soundboard, veneer, ribs, soundbox, access holes, string tension, etc.-interact with each other to produce an acceptable instrument. Ultimately one would like to create a set of desiderata for the design and manufacture of harp components in a similar manner to that already achieved for, say, the violin.

\section{1:15}

3pMU2. The nonlinear motion of harp strings. Chris Waltham and Andrzej Kotlicki (Dept. of Phys. \& Astron., University of British Columbia, Vancouver BC, Canada V6T 1Z1, cew@phas.ubc.ca)

The harp is an instrument with a set of plucked strings that excite the sound board directly, without the medium of a bridge. The strings are positioned at an acute angle to the plane of the sound board. The quality of the sound produced depends on the motion of the string and its interaction with the resonances of the sound board. String and sound board motions have been studied using small, infrared position sensors. The results are compared to those of a simple nonlinear model based on the measured elastic properties of the string materials and those of the soundboard. We will also describe the construction and calibration of the position sensors. 
3pMU3. On Dagestan tamur acoustical features. Shamil Aliev (DagDisel Factory, Lenina str. 1, Kaspiisk, Dagestan, Russia), Ernst Myshinsky, and Alexander Pyshin (Krylov Inst., Saint-Petersburg, 196158, Russia)

The study of the tamur, a national Dagestan two-stringed musical instrument played by plucking with narrow body gouged from linden wood, is presented. Distinctive features of the tamur sound making process are described. The resonance properties of the tamur body, that is, the mechanical resonance system, are determined. Also the resonance properties of air volume inside the tamur body, that is, an acoustic resonator, are determined. An interaction of the tamur body mechanical vibration and air volume inside the body oscillation are discussed. Tamur body sound radiation dependencies on frequency are studied. The resonance properties of instrument strings and purity of their sounding are determined. The assessment of efficiency of transformation string oscillations into tamur sounding board oscillations (body mechanical impedance) is discussed. The elastic and acoustic constants of linden, that is, the wood material of the tamur body made by gouge, are determined. The logarithmic decrement of tamur sounding board oscillations is determined. The typical spectra of tamur sounding are analyzed.

\section{$1: 45$}

3pMU4. Comparing harmonic reinforcement in the Octet's Mezzo and a typical Strad-model violin. Duane Voskuil (Univ. of North Dakota, Bismarck, ND, dvoskuil@bis.midco.net) and Carleen M. Hutchins (The New Violin Family Assoc., Wolfeboro, NH)

Many players and listeners maintain the Octet violins have a fuller sound than conventional instruments. Preliminary data indicate Octet violins may better embody some resonant intervals that make this more likely. A theory is presented based on a study comparing the Octet's Mezzo and a Strad-model violin, concluding that C. M. Hutchin's original design principle for the Mezzo fortuitously related A0 to A1 as an interval of a fifth near the two open middle strings, allowing more constructive wave reinforcement than conventional violins. The theory considers other intervals, seeking to maximize harmonic reinforcement by modifying instruments, (1) so that every bowed note is reinforced by an activated wood or air resonance by placing the notes fundamental and overtones close enough to a resonant frequency to activate it, (2) so that two resonances constructively interfere when they are simultaneously activated because they are separated by a harmonic interval, and (3) so that as many higher mode resonances as possible are activated by upper partials of the bowed notes harmonic series.

\section{2:00}

3pMU5. Linearized and nonlinear dynamics of bowed bars. Octavio Inacio (Escola Sup. de Musica e Artes do Espectaculo, Musical Acoust. Lab., R. da Alegria, 503, 4000-045 Porto, Portugal, octavioinacio@esmae-ipp.pt) and Jose Antunes (Instituto Tecnologico e Nuclear, 2686 Sacavem codex, Portugal)

Friction-excited instruments have been for many decades an inexhaustible source of physical delight. In the last decades, spectacular improvements in computational power and in numerical methods enabled simulations of the self-excited nonlinear regimes with considerable realism and detail. The authors of the present paper have achieved many such nonlinear simulations, using a powerful modal approach, and decided to investigate here in detail the characteristics of the linearized and nonlinear regimes of bowed bars. After stating the theoretical modeling approach for the nonlinear problem, we derive a corresponding linearized model, from which the complex eigenvalues and eigenvectors are computed as a function of the bowing parameters (friction parameters, normal force, and tangential velocity, accounting for the bowing location). We thus obtain plots of the modal frequencies, damping values, and complex mode shapes, as a of the system modes. When compared with the nonlinear motion regimes, these results offer interesting information concerning the stability behavior of the system, and further insight when addressing the postinstability nonlinear limit-cycle responses. Several differently tuned bars are addressed in order to assert the configurations leading to optimal playability.

\section{2:15}

3pMU6. Spectral method applied in physical modeling synthesis of plate. Lin Zhang (School of Phys., Univ. of Edinburgh, Kings Bldg., Edinburgh, EH9 3JZ, UK, lin.zhang@ed.ac.uk)

As a well-applied method in scientific computation, spectral methods have properties like higher order accuracy, nonlinearity expandability, and memory saving. By an example, this work will explore the potential of its application into physical-based sound synthesis With background work of finite difference on wave equation, spectral differentiation is further applied in space of discretization of partial difference equation (PDE). General plates (including membrane) percussion models are created numerically presenting the wave propagation inside of plate. According to two different dimensions (rectangular and circular), Fourier or Chebyshev interpolations are applied. In addition, stiff system is introduced by adding sizzlelike partials onto plate model (sizzled cymbals in jazz music) and complex collision phenomena (for example, ride cymbals). Problems such as stability condition take an important position in analysis. The algorithm is to generate high-quality multichannel audio outputs for musical purpose. For the continuity and expandability as application module, parallel computation programming is taken as the focus of final implementation on a Sun Bluefire E15K shared memory machine [Work supported by EPCC.]

\section{$2: 30$}

3pMU7. Vibrational characteristics of the glassharp. Koichi Uchida and Kenshi Kishi (Dept. of Electron. Eng., Univ. of Electro-Commun., 1-5-1 Chofugaoka, Chofu-shi, Tokyo, 182-8585 Japan coici@crane.ee.uec.ac.jp)

Using the finite-element method (FEM), glassharp vibration was analyzed. Results show that the glassharp pitch is controllable at the stage of manufacturing. First, the effects of several factors were studied, such as geometry approximation, element type, and the number of division on the accuracy of mode frequencies. Second, it was examined how each mode frequency changes by the fixed position and length of the glassharp stem. The results demonstrate that their influences were of no great importance. Third, the pitch was widely adjustable by changing the swell of the cup. Particularly, it was finely adjustable by cutting a small circular groove inside a bottom of the cup instead of a conventional method.

\section{2:45}

3pMU8. Physical modeling of Tibetan singing bowls using dynamic forcing. David M. Etlinger (School of Music, Northwestern Univ., 711 Elgin Rd., Evanston, IL 60208)

The geometric simplicity of Tibetan singing bowls produces surprisingly complex vibrational behavior. The curvature of the bowls causes strong coupling between modes, and the physical parameters of actual bowls (namely their thickness and deep curvature, as well as their asymmetrical excitation) produce higher-order effects that cannot be neglected. In this paper we present a physical model for the bowl's acoustical output. The model is based on analytical solutions for the natural modes, and predicts both transient and steady-state behavior. Using the method of dynamic forcing, we investigate bowl response for linear (striking) and nonlinear (rubbing) excitation methods, producing audio as well as numerical data. Finally, we use the model to gain insight into aesthetics and design: by comparing our simulations to actual antique or high-quality bowls, we can begin to determine what parameters contribute most to creating the desired sound. 
IAO NEEDLE/AKAKA FALLS ROOM, 1:00 TO 1:45 P.M.

\title{
Session 3pNSa
}

\section{Noise, Speech Communication, and ASA Committee on Standards: Progress in Hearing Protection and Hearing Conservation II}

\author{
Elliott H. Berger, Cochair \\ E-A-R Aearo Co., 7911 Zionsville Rd., Indianapolis, IN 46268-1657 \\ Hiro Takinami, Cochair \\ RION Co., Ltd., 3-20-41 Higashimotomachi, Kokubunji, Tokyo 185-8533, Japan
}

\section{Contributed Papers}

1:00

3pNSa1. Field study of sound exposure by personal stereo. Rodrigo Ordoñez, Karen Reuter, and Dorte Hammershøi (Dept. of Acoust., Aalborg Univ., Fredrik Bajers Vej 7-B5, DK-9220 Aalborg $Ф$, Denmark, rop@acoustics.aau.dk)

A number of large scale studies suggest that the exposure level used with personal stereo systems should raise concern. High levels can be produced by most commercially available $\mathrm{mp} 3$ players, and they are generally used in high background noise levels (i.e., while in a bus or train). A field study on young people's habitual sound exposure to personal stereos has been carried out using a measurement method according to principles of ISO 11904-2:2004. Additionally the state of their hearing has also been assessed. This presentation deals with the methodological aspects relating to the quantification of habitual use, estimation of listening levels and exposure levels, and assessment of their state of hearing, by either threshold determination or OAE measurement, with a special view to the general validity of the results (uncertainty factors and their magnitude).

\section{1:15}

3pNSa2. Antioxidant use in the prevention of noise-induced hearing loss and ototoxic chemicals in the workplace. Sandra C. MacLean Uberuaga (Alaska Occupational Audiol. \& Health Services, 200 West 24th Ave., \#196, Anchorage, AK 99503)

There have been discoveries in auditory research as to how the cochlea is injured during noise exposure and how it may sometimes be possible to reverse or prevent acute noise damage with medications. There are two primary mechanisms of damage; mechanical, as delicate tissue structures are torn apart when loud blasts occur above 125-130 dB SPL, and metabolic exhaustion, which commonly results from habitual noise exposure. During metabolic exhaustion toxic waste products (free radicals) form as cells in the cochlea are stressed. Our bodies can react to noise trauma by presenting a defense of antioxidant enzymes. However, when these defenses are overwhelmed, the hair cell is subject to serious damage to its nuclear DNA, mitochondria, and membranes. This research has been promising in animal studies. We will discuss pharmacological agents that can be used by the ear to synthesize the toxic products. The next step is well-designed human clinical trials in occupational settings where extreme noise levels cause hearing loss despite the seemingly appropriate use of hearing protection devices (e.g., aircraft carrier decks, mining and petroleum industries). Chemicals and agents that can cause hearing loss in the workplace will also be reviewed.

\section{1:30}

3pNSa3. Combined effects of occupational exposure to noise and organic solvents on the auditory system. Ikuharu Morioka (School of Health and Nursing Sci., Wakayama Medical Univ., 580 Mikazura, Wakayama, 641-0011 Japan), Yoshihiro Minami, Nobuyuki Miyai, Kazuhisa Miyashita (Wakayama Medical Univ., Wakayama, Japan), Hiroichi Yamamoto (Osaka Occupational Health Service Ctr., Osaka, Japan), and Orawan Kaewboonchoo (Mahidol Univ., Bangkok, Thailand)

Noise exposure is the most common cause of hearing loss. In many work environments where organic solvent exposure occurs, a high level of noise exposure is also common. However, as to the ototoxic effects of organic solvents, the earlier studies focused on the exposure to an organic solvent alone. It is necessary to clarify the combined effects of exposure to noise and organic solvents at the simulated situation of the work environments. The objective of this study was to review the literature on the ototoxic effects of exposure to organic solvents combined with noise. Two organic solvents were selected: methanol, a widely used organic solvents in the occupational field, and styrene, also widely used and a human neurotoxicant. Judging from the results of animal experiments and epidemiologic studies, a synergistical interaction of noise with such organic solvents' ototoxicity was clarified even at the lower concentration where the organic solvent alone showed no ototoxicity in the experimental animals and at the concentration lower than the occupational exposure limits of organic solvents. Consequently, it is important to carefully access the occupational exposure limits when workers are exposed to organic solvents combined with noise, and to take measures against combined exposure. 
IAO NEEDLE/AKAKA FALLS ROOM, 1:55 TO 3:00 P.M.

\title{
Session 3pNSb
}

\section{Noise: Active Noise Control}

\author{
Yukio Iwaya, Chair \\ Tohoku Univ., Research Inst. of Electrical Communication, 2-1-1 Katahira, Aoba-Ku, Sendai 980-8577, Japan
}

Chair's Introduction-1:55

Contributed Papers

2:00

3pNSb1. Active noise control-like performance of a spherical reflector. Kunisato Seto, Md. Tawhidul Islam Khan, Kenbu Teramoto (Dept. Mech. Eng., Saga Univ. 1 Honjo-machi, Saga-shi, Saga 840-8502, Japan, kunisato@cc.saga-u.ac.jp), and Hiromitsu Ohta (Natl. Fishery Univ. Shimonoseki 759-6595, Japan)

The principle of cancellation of tonal noise by using a spherical reflector, the designing method of an appropriate reflector for canceling jet screech, the advantage of a spherical compared to a flat reflector for canceling a tonal noise, and a prospective application for an aircraft to protect the fuselage from acoustic fatigue are discussed. The thrust loss due to the new technique was also evaluated directly with a vertical wind tunnel. The change in the aerodynamic structure was optically observed by using a schlieren apparatus along with a high-speed video camera. [Work supported by grant of JSPS, Japan.]

\section{2:15}

3pNSb2. A study of subjective effect on active noise control system. Sayoko Utsumi, Eiji Murai, Yuko Watanabe, and Hareo Hamada (SIE, Tokyo Denki Univ., 2-1200 Muzai-gakuendai, Inzai, Chiba, 270-1382, Japan)

In recent years, the influence of various noises on human beings in our daily life has become a problem. Especially, the low-frequency noise has been considered to have a serious influence on work efficiency of people who are working under the noise exposure. On the other hand, active noise control (ANC) systems have achieved the efficient reduction noise, especially for the low-frequency noise. Actually, a number of successful ANC systems have been reported. Therefore, ANC systems may improve our work efficiency. In this paper, features of various noises are observed to get a representative value to be a sound source for subjective listening tests. Then, the effectiveness of the ANC system is evaluated to observe a physiological reaction of subjects. For that, response times to the shortterm memory task will be measured in three different conditions, such as usual condition, under the noise exposure, and that with a running ANC system.
$2: 30$

3pNSb3. Near-field mapping of pressure fields during active noise control of small axial cooling fans. Benjamin M. Shafer, Kent L. Gee, Scott D. Sommerfeldt, and Cole V. Duke (Brigham Young Univ., Provo, UT 84602)

In the past, tonal noise from small axial fans has been globally reduced using active noise control (ANC) with near-field error sensors placed according to a theoretical condition of minimized radiated power [K. L. Gee and S. D. Sommerfeldt, J. Acoust. Soc. Am. 115, 228-236 (2004)]. The theoretical model, based on mutual coupling of point sources, showed that pressure nulls exist in the near field when the radiated power is minimized. Error sensor placement at these locations should then optimize global ANC. This study comprises an experimental investigation in which the actual locations of these near-field pressure nulls have been measured over a two-dimensional grid with a linear array of microphones. The array consists of 25 quarter-inch microphones with half-inch spacing. This array has been used to map the pressure field from a $60-\mathrm{mm}$ cooling fan during ANC, in addition to a benchmark case, where a small loudspeaker has been mounted in place of the fan. The experimental results are compared to the theoretical pressure null locations in order to determine the efficacy of the point source theoretical model.

\section{2:45}

3pNSb4. Active control of free-field sound using near-field energybased error signals. Ryan $T$. Chester and Timothy W. Leishman (Acoust. Res. Group, Dept. of Phys. and Astron., Brigham Young Univ., Provo, UT 84602)

Practical efforts to actively control sound often require error sensors located in the acoustic or geometric near field of primary and secondary sources. Unfortunately, when using conventional acoustic pressure sensors, control performance often becomes very sensitive to sensor position, and optimal sensor positions are difficult to ascertain or maintain with changing system conditions. Other types of error sensors or combinations of error sensors may produce fewer position-dependent complications, yielding excellent global sound-field control with greater ease and consistency. This paper compares the use of potential, kinetic, and total energy density error signals in the near field of a primary and secondary source in free space. It also discusses the distinct spatial and spectral uniformities of these quantities and their impact on the active control of sound. 
Session 3pNSc

\title{
Noise, Architectural Acoustics, and Speech Communication: Acoustical Design Factors in Spaces Where Speech Communication is Critical
}

\author{
Todd R. Beiler, Chair \\ 343 Lapa Pl., Kailua, HI 96734 \\ Chair's Introduction-3:10
}

Contributed Papers

3:15

3pNSc1. Can operating room nurses accurately classify noise exposures? Bernadette Stringer and Ted Haines (Dept. of Clinical Epidemiology and Biostatistics, McMaster Univ., ON, Canada)

Worker's qualitative chemical exposure estimates when compared to estimates made by experts or with monitoring devices have been found to be accurate. But almost no research exists on worker's ability to classify noise exposure. This investigation took place within a multi-hospital intervention study in which OR nurses completed a questionnaire at the end of each surgery and answered, "During this surgery could you easily hear: quiet talking, normal talking, or loud talking?" In 255 surgeries, noise was measured using a sound level meter, for a minimum of $15 \mathrm{~min}$, while in 68 surgeries noise was measured for $>70 \%$ of the surgery. In the 255 surgeries in which noise was measured for $15 \mathrm{~min}$ or $>$, it was found that nurses who heard quiet talking were exposed to $62.8 \mathrm{~dB}(\mathrm{~A})$ and that nurses who heard normal or loud talking were exposed to $65.1 \mathrm{~dB}(\mathrm{~A})$, a difference that was statistically significant $(p=0.019)$. In the 68 surgeries where noise measurements lasted more than $70 \%$ of the surgery, nurses who heard quiet talking were exposed to $64.0 \mathrm{~dB}(\mathrm{~A})$, while nurses who heard normal or loud talking were exposed to $67.3 \mathrm{~dB}(\mathrm{~A})$, a difference that was borderline significant $(p=0.07)$. Nurses can distinguish between noise that interferes with quiet talking during surgery and noise that does not. [Work funded by Ontarios Workplace Safety Insurance Board.]

3:30

3pNSc2. Noise in Canadian hospital operating rooms. Ted Haines and Bernadette Stringer (Dept. of Clinical Epidemiology and Biostatistics, McMaster Univ., ON, Canada)

Noise levels in hospital operating rooms have not been well characterized. Therefore, noise levels were measured using a sound level meter in a sample of surgeries included in a multihospital intervention study, assessing the effectiveness of a recommended work practice to decrease occupational exposure to blood during surgery. The duration of the measurements ranged from $15 \mathrm{~min}$ to several hours. Among types of surgery for which at least four measurements were done, the $\mathrm{L}_{\mathrm{eq}}$ for orthopedic surgery was the highest at $70.1 \mathrm{~dB}(\mathrm{~A})$ (range $60.8-75.1$ ), followed by 63.7 $\mathrm{dB}(\mathrm{A})$ for neurosurgery (range 57.4-68.1), and $62.8 \mathrm{~dB}(\mathrm{~A})$ (range 58.570.3), for general surgery. Gynecological surgery had the lowest $\mathrm{L}_{\text {eq }}, 60.8$ $\mathrm{dB}(\mathrm{A})$ (range 56.5-62.5). Peak levels were found to be as high as 132.8 and $132.6 \mathrm{~dB}(\mathrm{~A})$, in general and orthopedic surgery, respectively, and lowest in neurosurgery at $102.6 \mathrm{~dB}(\mathrm{~A})$. These noise levels are consistent with those from a comprehensive U.S. study, and substantially exceed EPAs recommended level of $45 \mathrm{~dB}(\mathrm{~A})$ for hospitals. [Work funded by Ontarios Workplace Safety Insurance Board.]
$3: 45$

3pNSc3. Noise levels with the potential to impede communication during surgery. John Oudyk and Bernadette Stringer (Occupational Health Clinics for ON Workers Inc., 848 Main St. E., Hamilton, ON L8M 1L9, Canada)

Surgical teams consist of personnel who may or may not regularly work together, among whom communication can be more difficult because of surgical masks, face shields, or goggles, factors believed to have contributed to a recent serious medication error that occurred when the anaesthetist misunderstood the surgeon's request. In a multihospital, occupational health intervention study a sound level meter was used to measure ambient noise for $15 \mathrm{~min}$ or $>$ during 256 surgeries included in the study. Results revealed an $L_{\text {eq }}$ of $70.7 \mathrm{~dB}(\mathrm{~A})$ and peak of $115.8 \mathrm{~dB}(\mathrm{~A})$ (at the 95th percentile). Since the clarity of words depends on maintaining a signal-to-noise ratio of at least $15 \mathrm{~dB}(\mathrm{~A})$, personnel working with such noise would likely increase their speaking levels up to $75-85 \mathrm{~dB}(\mathrm{~A})$, a level substantially higher than normal speech ranging from $55-65 \mathrm{~dB}(\mathrm{~A})$. Although the effect of noise levels on patient safety, or the health of OR personnel who must raise their voices to be heard, as well as on the overall climate that results when noise is elevated, have not been thoroughly investigated, evidence of negative effects is growing. And, while further characterization of OR noise is justified, this should be carried out to develop tailored interventions that can then be evaluated. [Work was funded by Ontario's Workplace Safety Insurance Board.]

4:00

3pNSc4. Comparison of speech communication of renovated classroom by subjective method. Coriolanus Lam, Chun Wah Leung (Dept. of Mech. Eng., The Hong Kong Polytechnic Univ., Hung Hom, Hong Kong, coriolanus.lam@polyu.edu.hk.), and Kai Ming Li (Purdue Univ., IN)

This paper presents measurement results on the acoustical characteristics of two standard and identical classrooms, one of which has been renovated. Several acoustical quantities that affect speech communication have been studied. These quantities were used to predict and quantify the characteristics and the qualities of measured classrooms that focused on speech communication. Subjective method by speech intelligibility tests was conducted in the measurements. Phonetically balanced monosyllabic word tests in both English and Chinese were carried out. In addition, public address messages, both in English and Chinese, from Hong Kong Mass Transit Railway were used as material for the tests. The results indicate that the renovation was beneficial on the quality of speech communication in general except for echo effects and increase in reverberation under several combinations of acoustical settings. These results were used to evaluate the relationship between speech perception quality and speech intelligibility. Besides, it will be used for new classroom designs in Hong Kong for better speech communication. [Work supported by Research 
Grants Council of The Hong Kong SAR Government, Research Committee of The Hong Kong Polytechnic University, and Architectural Services Department of The Hong Kong SAR Government.]

\section{4:15}

3pNSc5. Case study: Predict acoustical quality of renovated classroom. Coriolanus Lam, Chun Wah Leung (Dept. of Mech. Eng., The Hong Kong Polytechnic Univ., Hung Hom, Hong Kong, coriolanus.lam@polyu.edu.hk), and Kai Ming Li (Purdue Univ., IN)

The study on prediction of acoustical quality of two standard and identical classrooms, one of which has been renovated, was presented. Acoustical back-wall panels and sound-reflecting panels were installed in the renovated classroom. The study was carried out by RAYNOISE under the same conditions and acoustical characteristics of field measurements in the classrooms. Several acoustical quantities such as signal-to-noise ratios, reverberation time, and speech transmission index have been studied. Comparison was focused on the qualities of speech communication between the classrooms with and without renovation. Additional comparison was conducted with the results of field measurements. The results of the present study found that the quality of speech communication varied depending on changes in absorption. The results further implied the limitations and the precautions on application of RAYNOISE for different studies. [Work supported by Research Grants Council of The Hong Kong SAR Government, Research Committee of The Hong Kong Polytechnic University, and Architectural Services Department of The Hong Kong SAR Government.]

\section{$4: 30$}

3pNSc6. Speech privacy unmasked. David Lubman (14301 Middletown Ln., Westminster, CA 92683) and Louis C. Sutherland (Rancho Palos Verdes, CA 90275)

Background masking noise is widely used to achieve speech privacy between work stations in open offices and in nearby closed (executive) offices. Although speech privacy is often essential, high masking noise levels reduce the abilities of some workers to perform speaking and listening tasks effectively and without strain. Masking noise levels are usually set as high as office inhabitants will tolerate, typically about 45-48 $\mathrm{dB}$ (A-weighted). This is 10 to $20 \mathrm{~dB}$ higher than noise levels in typical home settings. Many who can communicate effectively in home settings are impaired in offices with background masking noise. People adversely impacted include those with mild or worse hearing disabilities of hearing, speech, and language. Many are older workers and non-native speakers. The current architectural trend to greater inclusiveness underscores the desirability of lower background masking noise levels. Designers can usually achieve speech privacy with lower masking noise levels by specifying partitions with higher noise isolation ratings. Higher sound isolation ratings cost more than higher masking noise levels. But higher costs may be balanced by the benefits of a more inclusive and humane workspace.

\section{$4: 45$}

3pNSc7. Objective and subjective evaluation of the acoustic comfort in public classrooms. Paulo H. Zannin, Fabiano B. Diniz, and Daniele Krauser (Mech. Eng. Dept., Federal Univ. of Parana, Rua Benedicto Berillo Fangueiro, 108, Santa Barbara, Curitiba, Parana, CEP, 81540-420, Brazil)

The acoustic comfort of classrooms in a Brazilian public school has been evaluated through interviews with 62 teachers and 464 pupils and measurements of background noise, reverberation time, and sound insulation. Acoustic measurements have revealed the poor acoustic quality of the classrooms. Results have shown that teachers and pupils consider the noise generated and the voice of the teacher in neighboring classrooms as the main sources of annoyance inside the classroom. Acoustic simulations resulted in the suggestion of placement of perforated plywood on the ceiling, for reduction in reverberation time and increase in the acoustic comfort of the classrooms. [Work supported by DAAD, CNPq and FINEP.]

\section{5:00}

3pNSc8. Experimental determination of end reflection loss (ERL) over a range of common types and configurations of duct terminations. Alexander P. Michaud and Kenneth A. Cunefare (771 Ferst Dr., Rm. 205, Atlanta, GA 30332)

The two-microphone impedance tube method ASTM E1050 was applied to directly measure the termination impedance of rectangular duct configurations of different aspect ratios and end conditions over a 25- to $500-\mathrm{Hz}$ frequency band. Measured values of the acoustic impedance, and hence the reflection coefficient, of the duct termination permits computation of end reflection loss. ERL is essentially the insertion loss of duct termination. The measurement program is challenging, as the precision required for accurate ERL computation is much higher than that for accurate determination of the reflection coefficient. The measurement program is part of a project to assess the available published data on duct ERL. The ASHRAE Handbook contains a tabulation of ERL data derived from predictions of the radiation impedance of a circular plane piston, and there have been some validation measurements made on a limited set of circular duct configurations and orifice plate terminations. Results to date closely conform to published ERL values. [Work supported by ASHRAE.]

\section{Contributed Poster Papers}

Posters will be on display from 1:00 p.m. to 5:30 p.m. Authors will be at their posters from 5:15 p.m. to 5:30 p.m.

3pNSc9. Classroom acoustics: Future criteria for the design and renovation of learning spaces. Sooch San Souci, Line Guerra, Nicolas Teichner (AiA-Audition, Intelligibility, Acoust., 47 rue Le Corbusier, 92100, Boulogne, France, sooch.sansouci@aiacoustique.com), and Dick Campbell (Bang-Campbell Assoc., Falmouth, MA)

Recent literature suggests that some of today's criteria for classroom acoustics might be in need of revision. This article explores the traditional acoustic classroom criteria as they are applied today, and why several of the design goals for construction or renovation of learning spaces might not be appropriate acoustic targets. The acknowledged quest for optimized acoustics for the enhancement of learning is discussed and related to a series of newer metrics for the measure and modeling of speech level, occupied and unoccupied noise, controlled reverberation, and SNR values presented on a seat-by-seat basis. Based on the measured results of classrooms designed and built in accordance to these newer metrics, our research indicates that these guidelines would incur an average increase of
$2 \%$ to $8 \%$ to current budgets. Specifics involving building costs versus acoustic value are also discussed. Further, research involved in the evaluation of scholastic advantages is underway.

3pNSc10. Speech and noise levels in classrooms-signal, noise, reverberation levels as a metric for acoustic design for learning. Line Guerra, Nicolas Teichner, Sooch San Souci (AiA-Audition, Intelligibility, Acoust., 47 rue Le Corbusier, 92100, Boulogne, France, line.guerra@aiacoustique.com), and Dick Campbell (Bang-Campbell Assoc., Falmouth, MA)

This study attempts to investigate the variance of speech levels in occupied classrooms during learning activities and their relationship to the total noise component (occupied, ambient, and reverberant). The methodology for the construction of a valid data base is presented, which would 
apply to criteria such as SNR (signal-to-noise ratio) and correlate to the term SNRL (signal, noise, reverberation levels). The interrelationship of speech and noise in any learning environment requires that the common variables associated with a stated speech level value should be stated along with the speech level value. The significance of the influences on the global value and in some cases the spectra of speech is discussed relative to variables such as the activity, the total occupancy and comportment of the audience, the volume of the room, total occupied noise in proximity to the speaker, speech directivity, effects of head diffraction among seated audience, and the distance from the source. Results from measurements taken in this study in actual classrooms are presented with an emphasis on specificity. This research has produced a strategy for the mapping of the speech-noise environment that significantly supports the use of a modeled SNR value as a goal for design.

THURSDAY AFTERNOON, 30 NOVEMBER 2006

WAIANAE ROOM, 1:00 TO 4:15 P.M.

\title{
Session 3pPA
}

\section{Physical Acoustics: Cavitation II}

\author{
Claus-Dieter Ohl, Cochair \\ Univ. of Twente, Faculty of Science and Technology, P.O. Box 217, 7500AE Enschede, The Netherlands \\ Shigeo Hayashi, Cochair \\ Univ. of Electro-Communications, 1-5-1 Chofugaoka, Chofu, Tokyo 182-8585, Japan
}

Invited Papers

1:00

3pPA1. Shock-wave-inertial-bubble interaction near an elastic boundary. Georgy Sankin and Pei Zhong (Dept. of Mech. Eng. and Mater. Sci., Duke Univ., Box 90300, Durham, NC 27708)

The interaction of laser-generated single cavitation bubbles (maximum diameter $D_{\max }=1.34 \mathrm{~mm}$ ) near a silicon rubber membrane with a shock wave (39-75 MPa in peak pressure and $1.7 \mu \mathrm{s}$ in compressive pulse duration) is investigated. The interaction leads to directional, forced collapse of the bubble with microjet formation along shock wave propagation direction. Maximum jet penetration into the membrane is produced during the bubble collapse phase with optimal shock wave arrival time and stand-off distance $s$, a condition that ensures that the forced collapse time of the bubble matches with the compressive duration of the shock wave to generate presumably the maximum axial jet velocity toward the boundary. A maximum penetration depth of $0.5 \mathrm{~mm}$ is observed at dimensionless proximity $\gamma\left(=2 s / D_{\max }\right)=0.7$ when the bubble establishes contact with the boundary during expansion. At larger $\gamma$ $(\geqslant 1.2)$, jet impact is significantly attenuated by the interposing water layer separating the collapsing bubble and the membrane surface. Such interaction may provide a novel acoustic means for in vivo microinjection, applicable to targeted delivery of macromolecules and gene vectors to biological tissues.

\section{$1: 20$}

3pPA2. In-liquid plasma processing using high-frequency or microwave irradiation. Shinfuku Nomura and Hiromichi Toyota (Dept. Mech., Ehime Univ, Matsuyama, 790-8577, Japan, nomu@eng.ehime-u.ac.jp)

In-liquid plasma is generated by applying high-frequency $(27 \mathrm{MHz})$ or microwave $(2.45 \mathrm{GHz})$ radiation to hydrocarbon liquids or water. In-liquid plasma is expected to replace gas-phase plasma because the reaction rates for chemical deposition of in-liquid plasma are much higher than those for conventional vapor deposition. Moreover, the cooling effect of the liquid mitigates thermal damage to the substrate. In-plasma generation requires the existence of bubbles because this plasma occurs in bubbles in liquid. Two methods exist for generating bubbles. One is to generate vapor bubbles by heating the liquid. The other is to generate acoustic cavitaion bubbles. Sonoplasma is created when acoustic cavitation is used to generate in liquid plasma. This plasma is not arc-discharged plasma, but rather high-frequency or microwave plasma. Therefore, it is useful in many industrial applications. Using this technology as a chemical reactor, synthesis of diamondlike carbon, polycrystalline silicon carbide, and carbon nanotubes, decomposition of waste oils, and the production of hydrogen can be achieved easily in hydrocarbon liquids. Temperatures were measured from the ratio of emission intensities of $H_{\alpha}$ and $H_{\beta}$. Plasma temperatures in hydrocarbon liquids are higher than those in water.

\section{$1: 40$}

3pPA3. Acoustical and optical characterization of air entrapment in piezo-driven inkjet printheads. Detlef Lohse, Jos de Jong, Roger Jeurissen, Michel Versluis (Phys. of Fluids, Univ. of Twente, P.O. Box 217, 7500 AE Enschede, The Netherlands), Hans Reinten, Marc van den Berg, and Herman Wijshoff (Océ Technologies B.V, 5900 MA Venlo, The Netherlands)

Stability of inkjet printers is a major requirement for high-quality printing. However, in piezo-driven inkjet printheads air entrapment can lead to malfunctioning of the jet formation. A voltage pulse applied to a piezoelectric element causes an ink-filled channel to deform, thereby creating a pressure waveform in the ink. Fluid acoustics are involved to guide the waveform energy towards the nozzle and to create pressure and velocity profiles needed for the droplet jetting process. Droplets are jetted every $50 \mu \mathrm{s}$, and the typical nozzle size is $30 \mu \mathrm{m}$. The piezo actuator is employed to actively monitor the channel acoustics and to identify distortions at 
an early stage. Modifications of the response of the piezo actuator indicate entrapped air bubbles and these allow us to investigate them. When the signal is employed as a trigger for high-speed imaging, the consequences of the entrained bubbles on the droplet formation can be visualized. [This study has been financed by the Fundamenteel Onderzoek der Materie (FOM) of The Netherlands under Grant. No. 02MFS39 and by Océ Technologies B.V.]

\section{Contributed Papers}

\section{2:00}

3pPA4. Fully nonlinear model for spherical bubble oscillations between rigid parallel plates. Jianying Cui and Preston S. Wilson (Mech. Eng. Dept., Univ. of Texas, Austin, TX 78712-0292)

Many medical ultrasound applications involve the interaction of intense acoustic waves with bubbles confined by blood vessels or tissue. As a step toward understanding such systems, a bubble confined between two parallel rigid plates was modeled using the image method. The model equation is of Rayleigh-Plesset form, including compressibility of the liquid and accounting for boundary conditions imposed by the plates [Cui et al., Proc. 17th ISNA, edited by Atchley et al. (AIP, New York, 2006), pp. 229-232]. An analytical solution for the second harmonic, and fully nonlinear solutions in the quadratic approximation, were reported previously. Here, a hybrid time-frequency algorithm is presented for solving the complete nonlinear model equation. In particular, the effect of the plates is most conveniently dealt with in the frequency domain, whereas the nonlinearity due to the gas law is best handled in the time domain. The procedure was verified for moderate drive amplitudes by comparison with a spectral algorithm for solving the model equation in the quadratic approximation [Cui et al., J. Acoust. Soc. Am. 118, 1942 (2005)]. Results obtained from the hybrid algorithm will be presented that illustrate the effect of plate separation for different amplitudes and frequencies of acoustic excitation.

\section{2:15}

3pPA5. Controlled cavitation in lab-on-a-chip systems. Claus-Dieter Ohl, Ed Zwaan (Phys. of Fluids, Univ. of Twente, Postbus 217, 7500 AE Enschede, The Netherlands), Sèverine Le Gac (Univ. of Twente, $7500 \mathrm{AE}$ Enschede, The Netherlands), and Kinko Tsuji (Shimadzu Europa GmbH, Duisburg, Germany)

Typically cavitation bubble dynamics is studied either in a free, liquid or close to rigid, free or flexible boundaries. Here, we report on a new experimental approach to study cavitation bubbles in confined geometries with sizes comparable to the cavitation bubble $(20-100 \mu \mathrm{m})$. These fluid environments are fabricated in a polymeric material (PDMS, polydimethylsiloxane) using a conventional molding process from a silicon master (lab-on-a-chip systems). The bubbles are generated by focusing pulsed laser light into the microfluidic systems filled with a light-absorbing dye. The bubble dynamics is recorded stroboscopically and with high-speed photography. We report on bubble dynamics in various geometries such as in triangular, square, and disk-shaped structures. Additionally, we study a cavitation bubble-based micropump, the effect of the channel width on jetting dynamics, and the interaction of the cavitation bubble on suspension cells. The fluid flow is easily visualized by adding microparticles into the liquid. The authors propose that this new experimental approach opens an interesting toolbox in cavitation research.

\section{2:30}

3pPA6. Model for the interaction between a bubble and an elastic particle. Todd A. Hay and Mark F. Hamilton (Appl. Res. Labs., Univ. of Texas, Austin, TX 78713-8029, haymaker@mail.utexas.edu)

At the Providence meeting, a model describing the interaction of a spherical bubble with a rigid spherical particle was presented [Hay and Hamilton, J. Acoust. Soc. Am. 119, 3408 (2006)]. Here, elasticity of the particle is considered. The model accounts for pulsation and translation and is accurate to fifth order in terms of the ratio of characteristic radius to separation distance. The dynamical equations are derived using the Lagrangian formalism of Ilinskii et al. [Proc. 17th ISNA, edited by Atchley et al. (AIP, New York, 2006), pp. 303-310]. Bubbles and elastic solid particles are considered with radii on the order of microns and driven at megahertz frequencies. The radial particle motion is quasistatic under these conditions. For moderate bubble pulsations at which the liquid may be considered incompressible, translational motion of the bubble and particle is insensitive to whether the particle is elastic or rigid. Consideration is also given to truncation of the model at fifth order. Derivation of the model equations to arbitrary order was facilitated by the development of a computer algebra program. The importance of including higher-order terms for small separation distances is illustrated. [T.A.H. was supported by an NSF Graduate Research Fellowship.]

\section{2:45}

3pPA7. Deformation of a single bubble in organic solutions by ultrasound. Hiroshi Itami, Shinobu Mukasa, shinfuku Nomura, Hiromichi Toyota, and Yoshiyuki Takahashi (Dept. of Mech. Eng., Ehime Univ., Matsuyama, 790-8577 Japan)

Behavior of a single bubble under an ultrasound resonant state in a hydrocarbon liquid such as n-dodecane or benzene was observed using a stroboscope and a charge-coupled device (CCD) camera. Although sonoluminescence was not confirmed because of bubble stability limitations, bubble deformation was observed at a situation of stability limitation. Deformation occurred when the bubble contracted to its minimum size. Numerical analyses of deformation growth by Rayleigh-Taylor instability of an oscillated bubble were carried out at the surface. The distortion amplitude of all degrees continued oscillating as the bubble expanded or contracted, and the oscillation frequency increased with the degree. The distortion amplitudes are amplified through the afterbounce by the resonance effect if the oscillation frequency of the distortion amplitude of some degree is approximately equal to the frequency of the afterbounce. The distortion amplitude of all degrees, except for amplified degrees, was dampened by the viscosity effect, and if the distortion amplitudes of all degrees were dampened, the bubble was considered to form a sphere and become stable. A phase diagram of stability of an oscillated bubble showed a function of an ambient bubble radius and the amplitude of forcing pressure.

\section{3:00-3:15 Break}

\section{3:15}

3pPA8. Observe multibubble cavitations in the ultrasonic cleaning tank. Nakagawa Noritoshi, Wu Chaoqun, and Sekiguchi Yasuhisa (Grad. School of Eng., Hiroshima Univ., 1-4-1 Kagamiyama, Higashi-hiroshima, Japan)

A cylindrical ultrasonic cleaning tank radiated by a PZT with a large horn operated at $27.3 \mathrm{kHz}$ is employed to investigate the manifestations of cavitation in the present research. The characteristics of this cleaning system are analyzed using experimental methods. In this cleaning tank, the nonuniformity of vibration velocity on the radiation surface induces the Eckart streaming, which can eliminate the effect of a standing wave on cleaning efficiency. Fresh tap water and degassed water are employed as test liquids in this research. In tap water, conical streaming is observed as the input voltage of the transducer is increased over $100 \mathrm{~V}$. The structure of conical streaming is anatomized. Its shape varies with the change of input voltage. In degassed water, ultrasonic fireworks are observed and special attention is paid to their subsequences, snowflake bubble clouds and water jet. 
3:30

3pPA9. Characterization of in-liquid plasma in a continuous liquid flow. Hiromichi Toyota, Shinfuku Nomura, Shinobu Mukasa, Hiroshi Yamashita (Dept. of Mech. Eng., Ehime Univ., Matsuyama, 790-8577 Japan, toyota@eng.ehime-u.ac.jp), Ayato Kawashima, and Tsunehiro Maehara (Ehime Univ., Matsuyama, 790-8577 Japan)

In-liquid plasma is a useful invention because its chemical reaction rate is several thousand times higher than those of conventional plasma techniques. However, in-liquid plasma is not so familiar now because its fundamental characteristics remain unclear. We investigated characteristics of in-liquid plasma in a continuous liquid flow to facilitate its application as a chemical decomposition or synthesis device. $N$-dodecane is supplied by the pump and a stable liquid flow is set in the rectangular vessel. Because the flow velocity is insufficient, a nozzle is set $2 \mathrm{~mm}$ distant from the microwave electrode tip. The average flow velocity of $n$-dodecane at the nozzle top is $0-19 \mathrm{~m} / \mathrm{s}$ and the Reynolds number is as high as 1.2 $\times 10^{4}$. A $2.45-\mathrm{GHz}$ microwave is introduced by a monopole antenna electrode inserted into a rectangular cavity resonator. In-liquid plasma is generated and observed in the flowing cavitation bubbles generated from the ultrasonic horn tip. The vessel's inner pressure is adjusted to $65-450 \mathrm{hPa}$. The temperature in the plasma reached $3600 \pm 300 \mathrm{~K}$; it was almost independent of the flow velocity because the physical properties of the plasma are influenced only slightly by the acoustic cavitation stream.

\section{$3: 45$}

3pPA10. Microbubble shape oscillations excited through an ultrasound-driven parametric instability. Michel Versluis (Phys. of Fluids, Univ. of Twente, P.O. Box 217, 7500 AE Enschede, The Netherlands), Peggy Palanchon, Dave Goertz, Nico de Jong (Erasmus MC, Rotterdam, 3000 DR Rotterdam, The Netherlands), Ivo Heitman, Sander van der Meer, Benjamin Dollet, and Detlef Lohse (Univ. of Twente, 7500 AE Enschede, The Netherlands)

An ultrasonically driven air bubble can become shape-unstable through a parametric instability. Here, we report time-resolved optical observations of shape oscillations (mode $n=2$ to 6 ) of micron-sized single air bubbles for a range of acoustic pressures. The observed mode number $n$ was found to be linearly related to the resting radius of the bubble. Above the critical driving pressure threshold for shape oscillations, which as expected is minimum at the resonance of the volumetric radial mode, the observed mode number $n$ is independent of the forcing pressure amplitude. The microbubble shape oscillations were also analyzed numerically by introducing a small, nonspherical linear perturbation into a Rayleigh-Plesset-type equation model which includes a physical thermal damping mechanism describing heat and mass transport in the thin boundary layer at the bubble-to-water interface. Indeed, a parametric instability is responsible for the shape oscillations, and the Rayleigh-Plesset-type equation captures the experimental observations in great detail.

4:00

3pPA11. Numerical investigation of interactions between ultrasound propagation and cavitation bubbles in shock wave therapy. Marko Liebler, Thomas Dreyer, and Rainer E. Riedlinger (Institut fuer Hoechstfrequenztechnik und Elektronik, Universitaet Karlsruhe, Kaiserstr. 12, D-76131 Karlsruhe, marko.liebler@ihe.uka.de)

Acoustic cavitation in shock wave applications is responsible for several therapeutic effects as well as for undesired side effects. Using a twophase continuum approach for bubbly liquids, a numerical model is presented to simulate the interactions between nonlinear ultrasound propagation and cavitation bubbles. Numerical results are presented, showing in what manner the presence of cavitation bubbles alters the pressure pulse propagation and how the bubble dynamics can be specifically affected by changing the shock pressure waveform. An increased bubble density leads to significant changes in the tensile part of the pressure waveform. This effect is caused by the changes in medium compressibility due to the oscillating cavitation bubbles. Calculations for the bubble cloud dynamics induced by three pressure waveforms with identical energy but different temporal forms of the tensile part are compared. Simulation results demonstrate that tensile parts with low amplitude but long duration increase the cavitation effect compared to high amplitude but short tensile phases.

\title{
Session 3pPP
}

\section{Psychological and Physiological Acoustics: Auditory Grammar}

\author{
Valter Ciocca, Cochair \\ Univ. of Hong Kong, 5 F Prince Philip Dental Hospital, 34 Hospital Rd., Hong Kong \\ Kazuo Ueda, Cochair \\ Kyushu Univ., Dept. of Applied Information and Communication Sciences, 4-9-1 Shiobaru, Minamiku, \\ Fukuoka 815-8540, Japan
}

Chair's Introduction-1:00

Invited Papers

1:05

3pPP1. Auditory grammar: The event construction model and spoken language. Yoshitaka Nakajima (Dept. of Acoust. Design, Kyushu Univ., 4-9-1 Shiobaru, Minami-ku, Fukuoka 815-8540, Japan, nakajima@design.kyushu-u.ac.jp)

Several auditory illusions are accounted for by using the event construction model as presented by Nakajima et al. (2000) in order to explain the gap transfer illusion. In this model, the perceptual system detects and processes onsets and offsets of sounds as if they were independent elements. Temporal edges of sounds and their perceptual attributes are combined to construct auditory events 
obeying the proximity principle in time and frequency. For example, the onset and offset of a Chinese syllable belonging physically to different tones can be combined if close enough in time and frequency. The listener often hears a syllable with a lexical tone resulting from this illusory connection. Illusory attribution of perceptual features also occurs in nonspeech tones. One demonstration where only an offset of a glide is presented shows the mechanism; a very short illusory tone with no physical counterpart is perceived. Probably, the perceptual system looks for an onset to connect with the offset, but fails and is forced to restore an onset. Our attempts to connect this model to speech perception and pitch perception are presented along with some novel demonstrations. [Work supported by JSPS and 21st Century COE, Kyushu University.]

\section{$1: 25$}

3pPP2. The 100- to 150-ms window of auditory temporal processing and its consequences. Pierre Divenyi (Speech and Hearing Res., VA Medical Ctr., Martinez, CA 94553)

Robert Efron (Percept. Psychophys. 14, 518-530 [1973]) demonstrated that the minimum duration of a percept is between 100 and $130 \mathrm{~ms}$ for auditory, vibrotactile, and visual stimuli, suggesting the presence of a most likely centrally located temporal integrator with a time window of about that length. Single-unit studies in the cat auditory cortex also unveiled an integration time of 100-150 ms (C. E. Schreiner and R. V. Urbas, Hear. Res. 21, 227-241 [1986]). This window corresponds to the lower limit of the longest among Ira Hirsh's three-tiered temporal processing ranges [in Sensation and Perception, edited by H. R. Moskovitz et al. (Riedel, Dordrecht, 1974), pp. 251-258]. The present study argues that a temporal window of this duration can account for a variety of auditory perceptual results requiring temporal processing, such as temporal order identification, segregation of dynamically changing streams, robust speech intelligibility, and backward masking. Results also show that this window routinely becomes longer with age, with the possibility that the increased length of the window may account for some well-known central auditory processing deficits in aging. [Work supported by NIH, the VA Medical Research, and the 21st Century COE Progam, Kyushu University.]

\section{$1: 45$}

3pPP3. Types of illusory continuity of verbal and nonverbal sounds: Some observations leading to their discoveries. Richard M. Warren (Univ. of Wisconsin-Milwaukee, Dept. of Psych., P.O. Box 413, Milwaukee, WI 53201-0413)

When gaps of a few hundred ms in a signal (e.g., speech, music, steady-state tones, tone glides) are replaced by a louder sound capable of masking the missing portion, the signal appears intact; the absent fragment can be perceptually synthesized through reallocation of a portion of the louder sound's auditory representation. General rules and the special rules governing various types of restoration will be discussed, as well as how they were found through a series of observations, inferences, and experiments. [Work supported by NIDCD Grant No. R01 DC000208, and travel expenses provided by the COE Program of Kyushu University.]

\section{2:05}

3pPP4. Music sound restoration and the effect of presentation conditions. Takayuki Sasaki (Miyagi Gakuin Women's Univ., Sendai, Japan)

When a music sound in a melody was deleted and replaced by a noise, a missing sound can be restored perceptually. This illusory phenomenon in audition, named music sound restoration, can be proved indirectly by inaccurate judgment of temporal position of the inserted noise. In the present study, music sound restoration was investigated in terms of the temporal position of replaced noise, headphone listening or free field listening, and the listeners' knowledge of the phenomenon. The results showed that the accuracy of the noise location differed between the positions of replaced noise in the melody or within a beat. The perceptual restoration occurred not only in a headphone listening but also in a free-field listening. It was revealed that the accuracy of noise localization elevated when the listeners knew the stimulus construction, but it was still lower than that of a blank. It was also confirmed that a missing sound could be restored perceptually, not only in a melodic sequence but also in a simple sequence such as scale. [This work was supported by JSPS and 21st Century COE, Kyushu University.]

\section{$2: 25$}

3pPP5. An analysis of timing microstructure in Mozart's piano sonata K.331. Kengo Ohgushi (1-6-10 Oheyama, Goryo, Nishikyo-ku, Kyoto 610-1102 Japan, ohgushi-19.38@r7.dion.ne.jp)

It is known that a phrase-final lengthening, including a pause, plays an important role both for the intelligibility and the naturalness of speech. This fact is likely to hold true for performance of music. The present study investigates (a) how the phrase-final lengthening appears in performances of Mozart's piano sonata in A Major (K.331) and (b) what are common and individual features of the performances by five groups of pianists, European professional, Chinese student, Korean student, Japanese professional, and Japanese student pianists. One hundred and twelve pianists' performances of the first eight measures are analyzed in terms of the inter-onset intervals (IOIs). The data are averaged for the respective groups. The IOI patterns indicate (a) the final notes of the first half measures as well as the final notes of the second half measures are lengthened, (b) final note lengthening is more prominent in the fourth measure where the first phrase ends than in the other measures, and (c) Japanese student pianists play the rhythm of dotted eighth note and the sixteenth note differently from the pianists of other countries. [Work supported by JSPS and 21st Century COE, Kyushu University.] 
3pPP6. Lost silence as a consequence of stream segmentation. Shuji Mori (Cognit. Sci. Lab., ISEE, Kyushu Univ., 6-10-1 Hakozaki, Higashi-ku, Fukuoka 812-8581, Japan)

A sequence of successive tones can be segmented into perceptually distinctive streams by inserting a silent gap or by changing the frequency in mid-sequence. This study demonstrates that a silent gap is perceptually lost when the gap coincides with the frequency change that occurs between tones. Even a 40-ms gap is undetectable when the tonal frequency is shifted from 800 to $3200 \mathrm{~Hz}$. One interpretation of this "lost silence" phenomenon is the prioritization of frequency change as a cue for auditory segmentation. A silent gap and a change in frequency both mark a stream boundary if they are salient. They will be redundant as boundary markers when they coincide with each other; the frequency change will preempt the priority in auditory processing, whereas gap information will receive little processing and eventually become indiscernible. This phenomenon might be related to perceptual ambiguity of the temporal relation of separate streams (Van Noorden, 1975). [Work supported by JSPS.]

\title{
Contributed Paper
}

\section{3:05}

3pPP7. Exclusive allocation of a gap that is shared by two separate sounds. Gerard B. Remijn and Yoshitaka Nakajima (Kyushu Univ., Faculty of Design, 4-9-1 Shiobaru, Minami-ku, 815-8540 Fukuoka, Japan)

When a short temporal gap (40 ms or less) is inserted at the crossing point of two separate sounds of unequal duration, the gap is often allocated only to the shorter sound, whereas the longer sound can be perceptually completed (modal completion). This study shows that the exclusive allocation of the gap to the shorter sound-and the perceptual completion of the longer sound-is most profound when the start of the short sound and the start of the gap are separated by $400 \mathrm{~ms}$ or less. A possible explanation for the exclusive allocation of the gap is that within this temporal window, attention is engaged in the formation of a new auditory stream, a process initiated by the shorter sound's onset. When subsequent auditory elements - even a gap-arrive within the temporal window of attentional engagement, these elements will be automatically included in the new stream. [This study was supported by the COE program of Kyushu University, Japan, and the Japan Society for the Promotion of Science.]

$3: 20-3: 45$

Panel Discussion

THURSDAY AFTERNOON, 30 NOVEMBER 2006

HONOLULU ROOM, 1:30 TO 4:10 P.M.

\section{Session 3pSA}

\section{Structural Acoustics and Vibration and Architectural Acoustics: Vibration and Impact Sound in Buildings}

\author{
Takashi Koga, Cochair \\ Kajima Corp., 1-2-7 Moto-Akasaka, Minato-ku, Tokyo 107-8388, Japan \\ James E. Phillips, Cochair \\ Wilson Ihrig and Associates, Inc., 5776 Broadway, Oakland, CA 94618-1531
}

Chair's Introduction-1:30

Invited Papers

$1: 35$

3pSA1. Japanese standards and situation of studies about heavy and soft impact sources. Atsuo Hiramitsu (Bldg. Res. Inst., 1 Tachihara, Tsukuba-City, Ibaraki 305-0802, Japan, hiramitu@kenken.go.jp) and Katsuo Inoue (Nihon. Univ, Kanda-Surugadai 1-chome, Chiyoda-ku, Tokyo 101-8308, Japan)

This report describes standards of heavy and soft impact sources. They are used for measurement of floor impact sound insulation of buildings. In Japan, the method for field measurement of floor impact sound level (JIS A 1418) was revised in 2000 and separated into two parts. Part 1 uses a standard light impact source and corresponds to ISO 140-7. Part 2 uses heavy and soft impact sources. At that time, a rubber ball was standardized along with a car-tire source in JIS A 1418-2. Moreover, JIS A 1440-1 and JIS A 1440-2 for laboratory measurement of the reduction of transmitted floor impact sound have been prepared. JIS A 1440-1 corresponds to ISO 140-8, and JIS A 1440-2 uses heavy and soft impact sources. Floor impact sources characteristics are introduced. They include force characteristics, temperature dependence, stability, and others. In addition, among contents of JIS A 1440-2, the measurement method, calculation method, and a laboratory of a box-frame-type reinforced concrete construction are explained. In addition, study examples using a rubber ball are introduced. As examples, we present the floor impact sound level, the transmitted floor impact sound level, hearing tests on floor impact sound, and evaluation of walking sensations. 
3pSA2. Trends for prediction methods of heavyweight floor impact sound. Takashi Koga (Bldg. Construction Management Div., Kajima Corp., 1-2-7 Moto-Akasaka, Minato-ku Tokyo 107-8388, Japan, tkoga@kajima.com), Katsuo Inoue (Nihon Univ., Tokyo 101-8308, Japan), and Shinji Nakazawa (Tekken Corp., Chiba, 286-0825, Japan)

Trends of building structures of Japanese multifamily dwellings and prediction methods of heavyweight floor impact sound are introduced. Floor impact sound-reduction performance caused by human action like stepping and jumping is considered to be a vital function for Japanese apartment complexes. There are quite a few cases where the floor thickness shall be designed from 200 to 300 $\mathrm{mm}$ because of acoustical performance, to assure the required performance. The practical prediction method, the so-called impedance method, was been established in the 1970s, and it has been commonly used. Though there are many buildings that are out of the applicable scope of the ordinal impedance method with the rise of structural design technology, the outline shown is that of the revised practical prediction method that is under discussion by the working group of the Architectural Institute of Japan. And, examples of hybrid method are also introduced that substitute the impedances by numerical vibration calculation results using finite-element method for simplified calculation results of impedance method.

2:15

3pSA3. Correlation of field measurements for footstep force pulse with finite element analysis model for determining vibration response of a building floor. Richard Carman and James Phillips (Wilson, Ihrig \& Assoc., 5776 Broadway, Oakland, CA 94618)

An idealized force pulse that simulates footsteps was developed by Galbraith and Barton. Their proposed forcing function was determined from direct force measurements using walkers of different weights walking at various speeds. Unger and White used this idealized footstep pulse to determine the maximum dynamic deflection of a floor for the purpose of building design calculations. These basic concepts were used to revisit the characteristics of the idealized forcing function. During the design phase of an academic research facility, extensive vibration measurements were obtained using an exemplar floor similar in design to that proposed for the new research building. The exemplar floor was modeled using FEA. Its modal characteristics were compared to the field test results and found to be in reasonable agreement. Upon having established confidence in the basic FEA model, the field measurement results of two walkers on the exemplar floor were compared with the FEA results predicted using the Galbraith-Barton footstep pulse. The shape and magnitude of the footstep pulse were iteratively adjusted until the measurement results and FEA results were in good agreement. The results of the FEA analysis and the measurement program behind the model will be presented.

2:35

3pSA4. Influence of lightweight structural design of office buildings on vibration levels due to people walking and operation of mechanical equipment. Steven D. Pettyjohn (The Acoust. \& Vib. Group, 5700 Broadway, Sacramento, CA 95820-1852)

West coast buildings typically use lightweight structural designs, even for Class A office building. This means the best floors use lightweight concrete on fluted decks with beam and column spacings of 30 to $35 \mathrm{ft}$. with open web joists at $10 \mathrm{ft}$. on center. Class B office building may use columns at 28 and $35 \mathrm{ft}$. with only two-layers of plywood on wood open web joists at 24 in. on center. The resulting floor/ceiling assemblies are structurally sound but dynamically very live. Some structural damping would be expected from the introduction of partitions, but with the use of large open areas and partial height partitions, damping is limited. These assemblies have relatively low resonance frequencies and are very susceptible to any sinusoidal input force such as people walking or operation of mechanical equipment. Vibration measurements, made on four buildings after complaints were received, show the source of some of these problems. The results are compared with predictions by Murray et al. and Ungar et al. The lack of composite action between the beams and joists/purlins and the floor appears to be the source of many of the problems. Solutions to the problems in these existing and occupied buildings are discussed.

2:55-3:10 Break

\section{Contributed Papers}

3:10

3pSA5. Heavy and light impact sources to rate impact sound transmission and changes due to applied floor toppings. Alf Warnock and Trevor Nightingale (Acoust. Group, Inst. for Res. in Construction, Natl. Res. Council Canada, Ottawa, K1A 0R6, Canada)

The magnitude and spectrum of the power injected by an impact source depends on the impedance of the source and floor. If standardized impact tests are meant to give ratings that correlate well with subjective impressions of footstep noise, it follows that the impact source used should have the same impedance as an average human foot at least over the range of test frequencies. The ISO tapping machine, the Japanese tire machine, and an $18-\mathrm{cm}$-diam rubber ball do not satisfy this criterion. Consequently, their impact spectra differ from those from a live walker. Floor toppings, in particular, are ranked differently. Examples of discrepancies will be presented for direct transmission between vertically separated rooms. For horizontally and diagonally separated rooms, flanking transmission controls the impact sound pressure level. The sound pressure level depends not only on the power injected by the source, but also source location relative to the flanking junction because of propagation attenua- tion across the floor. Sensitivity to source location is similar for different sources (ISO tapping machine and Japanese ball), suggesting that the same source could be used for direct and flanking transmission measurements if the source adequately simulates the impedance of a human foot.

\section{$3: 25$}

3pSA6. Evaluation of mid- and high-frequency impact insulation methods. John J. LoVerde and David Wayland Dong (Veneklasen Assoc., 1711 Sixteenth St., Santa Monica, CA 90404)

Impact insulation is currently measured using impact insulation class (IIC) and a standard tapping machine described in ASTM E492 and E1007 (and corresponding ISO metrics and standards). The resultant IIC value does not correlate well with subjective reaction. In previous papers, the authors have focused on the low-frequency thudding from footfalls in lightweight wood construction and shown that the impact sound pressure level (ISPL) in the $63-\mathrm{Hz}$ octave band correlates better with subjective reaction than IIC or other metrics [LoVerde and Dong, J. Acoust. Soc. Am. 112, 2201 (2002); INCE Inter-Noise 2004 Proceedings, 167 (2004); J. Acoust. Soc. Am. 119, 3220 (2006)]. Further, the low-frequency ISPL is 
controlled by different variables than the mid/high-frequency ISPL. This suggests that a two-metric system would be valuable where the first metric describes the low-frequency performance and the second metric describes the $\mathrm{mid} /$ high-frequency performance. Field testing experience is presented showing that IIC does not adequately differentiate the mid/high-frequency performance of various resilient materials. Candidate mid/high-frequency metrics are evaluated to determine if any result in improved methods for evaluation and rank-ordering of floor/ceiling assemblies.

\section{3:40}

3pSA7. Flanking impact sound transmission in wood-framed multifamily construction. David Quirt and Trevor Nightingale (Acoust. Group, Inst. for Res. in Construction, Natl. Res. Council Canada, Ottawa, K1A 0R6 Canada)

This paper reports findings from a recently completed study of flanking sound transmission involving the wall/floor junction in wood-framed multifamily buildings. Flanking transmission exists in all buildings, but it can be controlled through good design. The paper examines how common construction details affect flanking paths between horizontally, vertically, and diagonally separated rooms. Variables considered include mounting conditions and orientation of the floor joists, framing of the wall assembly and any associated fire blocking, and mounting and number of layers of gypsum board. Estimates of the apparent sound insulation were obtained by summing the energy transmitted by the direct path through the wall or floor assembly separating the pair of rooms, and all the flanking paths involving the relevant wall-floor junctions. Results indicate that if there is no floor topping (i.e., the subfloor is bare) the apparent sound insulation for both airborne and impact sources is typically limited by flanking transmission involving the floor. Since, the source room floor is an element common to all impact flanking paths, three different toppings were evaluated as treatment options, and additional layers of gypsum board and resilient mounting were considered as options for the walls. The effectiveness of each option is discussed

$3: 55$

3pSA8. Case history building structural vibration isolation for $\mathbf{5 0 0}$ Atlantic Avenue. Gregory Tocci (Cavanaugh Tocci Assoc., Inc., 327F Boston Post Rd., Sudbury, MA 01776), George Wilson, James Phillips (Wilson Ihrig \& Assoc., Inc.), Gladys Unger, and James Moore (R.H. Lyon Div. of Acentech)

Vent Building 3 of the new Central Artery Tunnel located at 500 Atlantic Avenue in Boston, Massachusetts is a heavy poured in place concrete structure with its roof situated about $3 \mathrm{ft}$. below grade. The vent building contains $23100+$-in.-diameter double-width centrifugal fans that, for a number of reasons, are not vibration isolated. The fans exhaust into a cluster of reinforced concrete shafts that rises up to about $250 \mathrm{ft}$. above grade. The tall stacks have been embedded in a luxury hotel/ residential condominium complex that Extell Development Company is currently constructing. The stacks provide both vertical support and seismic support for the building. This paper summarizes the history of work on the project, and compares predicted and measured sound and vibration levels in representative building spaces. [We wish to acknowledge the support of Extell Development Company, Boston, MA for their support of this work.]

\title{
Session 3pSC
}

\section{Speech Communication: Speech Timing and Pronunciation Training for the Japanese Language}

\author{
Robert F. Port, Cochair \\ Indiana Univ., Linguistics, 330 Memorial Hall, Bloomington, IN 47405 \\ Yukari Hirata, Cochair \\ Colgate Univ., Dept. of East Asian Languages and Literature, 13 Oak Dr., Hamilton, NY 13346 \\ Chair's Introduction-1:00
}

Invited Papers

1:05

3pSC1. Timing variations of frequent utterances occurring in a very large corpus of spontaneous Japanese conversational speech. Nick Campbell (ATR, Kyoto, Japan)

A corpus of spontaneous conversational Japanese speech was collected from volunteer subjects who wore high-quality headmounted microphones and recorded their daily spoken interactions to minidisk over a period of 5 years. All recordings were transcribed and tagged according to interlocutor type, and a portion representing about $10 \%$ was further annotated for speech-act, speaker-state, emotion, and speaking style. This paper presents timing data from the corpus, showing how the same utterance can vary according to speaking style and other factors. It presents the hundred most common utterances in the corpus and relates their durations to spectral and prosodic characteristics that vary according to affect, attitude, intention, and relationship with the listener.

\section{$1: 25$}

3pSC2. On the generation and perception characteristics of Japanese timing control. Yoshinori Sagisaka (Waseda Univ. 29-7 Bldg., 1-3-10 Nishi-Waseda, Shinjuku-ku, Tokyo 169-0051 Japan, sagisaka@giti.waseda.ac.jp), Makiko Muto (Univ. of Tokyo, Japan), Hiroaki Kato (NiCT/ATR, Japan), and Minoru Tsuzaki (Kyoto City Univ., Japan)

We have been studying temporal characteristics of Japanese for decades. Not only acoustic measurements but also perceptual studies on temporal modification have revealed the control principles lying behind manifestation of segmental duration characteristics. This talk tries to introduce familiar generation principles such as "mora-timing" and phrasal tempo reset from the viewpoint of humans temporal organization through the understanding of duration statistics. Moreover, by showing perceptual characteristics 
including sentential factors, we would like to propose how linguistic units relate to temporal perception and what kind of information form can be considered in temporal cognition process. Some of our recent efforts will also be presented to investigate languagedependent temporal characteristics by observing non-native learners temporal control characteristics. We really expect that we will be able to have a better picture of generation and perception mechanism through the cross check of their characteristic differences between natives and non-natives. [Work supported by Waseda Univ. RISE research project of "Analysis and modeling of human mechanism in speech and language processing" and Grant-in-Aid for and Scientific Research A-2, No. 16200016 of JSPS.]

\section{$1: 45$}

3pSC3. Temporal control in Japanese dialects. Haruo Kubozono (Kobe Univ., Nada-ku, Kobe 657-8501 Japan, kubozono@lit.kobe-u.ac.jp)

This paper discusses the extent to which temporal control varies among Japanese dialects. Most dialects of Japanese including Tokyo Japanese are so-called "mora dialects" in which the mora serves as the basic unit of timing. In the periphery of Japan, however, we find a couple of dialects called "syllable dialects" whose basic phonological unit is the syllable rather than the mora. Kagoshima Japanese (KJ) is one such dialect, but little is known about its temporal organization. The main purpose of this paper is to report on the results of an acoustic experiment on this syllable-based dialect and to compare them with those of the experiments about Tokyo Japanese (TJ). This comparison reveals the following four points: (i) geminate consonants are considerably longer than single consonants in both $\mathrm{TJ}$ and $\mathrm{KJ}$, (ii) however, the phonetic duration of words in $\mathrm{KJ}$ is not determined by the number of moras involved but by the number of syllables, (iii) in KJ, vowels are consistently shorter when they follow geminate consonants than when following single consonants, and (iv) unlike English, Korean, and other languages, neither TJ nor KJ shows an effect of vowel shortening in closed syllables.

\section{2:05}

3pSC4. Japanese mora-timing and processing: The case of devoiced vowels. Natasha Warner and Naomi Ogasawara (Dept. of Linguist., Univ. of Arizona, P.O. Box 210028, Tucson, AZ 85721-0028, nwarner@u.arizona.edu)

An extensive literature has argued that Japanese speakers compensate for inherent durational differences to keep moras to approximately equal lengths (e.g., Han, 1962; Port et al., 1980; Minagawa-Kawai, 1999; reviewed in Warner and Arai, 2001a). However, near-regularity of moras may not be because of intentional compensation (Warner and Arai, 2001b). This paper examines Japanese listeners processing, rather than production, of one case of irregularly timed moras: vowel devoicing. When Japanese vowels are devoiced, the mora tends to be shorter than usual. If speakers aim for regular timing, one might predict that listeners would also expect moras to be regularly timed and would thus have difficulty processing sequences that deviate from regular mora timing. However, one could predict that listeners are aware of what deviations from regular timing to expect, and thus have no difficulty processing timing deviations. The results, from phoneme monitoring and lexical decision tasks, show that listeners only have difficulty processing devoiced vowels in environments where devoicing would not be expected. In fact, where devoicing would be typical, listeners find devoiced vowels easier to process. This suggests that in processing, knowledge of patterns in the language outweighs any bias toward regularly timed moras.

3pSC5. Perception and production of long and short vowels in Japanese by children. Takayuki Arai (Dept. of Elec. and Electron. Eng., Sophia Univ., 7-1 Kioi-cho, Chiyoda-ku, Tokyo, 102-8554 Japan), Kyoko Iitaka, and Eriko Ohki (Sophia Univ., Chiyoda-ku, Tokyo, 102-8554 Japan)

Studies have shown that acquisition of reading depends on such phonological processes as verbal short-term memory and temporal processing of speech. An inability to encode phonological representations orthographically may cause reading disabilities, such as dyslexia. Categorical perception is known to be closely associated with phonological encoding. Thus, studies examining the temporal processing of speech sound categorization may be useful in differential diagnoses of reading development and disabilities. The first study examines the perception of long and short Japanese vowels by a group of seven normal six-year-old children and two with delayed phonological development. Subjects were asked to identify vowel length in sets of words. The first vowel in each stimulus word was made to vary with respect to length. Perception by normal and delayed children differed in ways similar to what had been reported in previous English studies. In the second study, the relation between perception and reading of long/short vowels was examined by a group of 125 children ages 4 to 8 . Similar settings were used for the second experiment. The results showed that (1) a large developmental change of perception was observed in ages $6-8$ and (2) a positive correlation was obtained between perception and reading tasks.

\section{2:45}

3pSC6. Effects of speaking style on the regularity of mora timing in Japanese. Michael Connolly Brady, Robert F. Port, and Kyoko Nagao (Dept. of Linguist., Indiana Univ., Bloomington, IN 47405)

The regularity of mora timing in Japanese has remained controversial over the years [Warner and Arai, Phonetica 58, 1-25 (2001)]. It is possible that the degree of regularity varies with speaking style. Four Japanese subjects spoke six-mora proper names with varying syllable structures where the second name had either three simple syllables (e.g., Tomiko) or two syllables with a long vowel (e.g., Tooko) or a long consonant (e.g., Tokko). These were read either in formal sentences, read in conversational style sentences, or used in spontaneous description of pictures of characters having these names. Moras were measured as the intervals between vowel onsets using an automatic vowel-onset detection algorithm. Although all styles suggested regular mora timing, the results show that the styles differ in the degree of temporal compensation for the moras constituted by long vowels and long consonants which are shorter than consonant-vowel syllables. The compensation was clearest in the most formal style of speech. 
3pSC7. Representations of within-word structure and Japanese speech timing. Takashi Otake (E-Listening Lab. and Max Planck Inst. for Psycholinguist., 3-35-20, Higashi-Tokorozawa, Tokorozawa, Saitama, 359-0021 Japan)

One complication about the debate of mora timing is that durational units are closely related to syllable structure in which morae can be treated as subunits of syllables. As a consequence, durational properties of the components in three types of syllables, CVN, $\mathrm{CVV}$, and CVQ, can equally be examined from the point of representations of within-word structure. Durational properties in Japanese speech timing undoubtedly play an important role, but knowledge of representations of within-word structure cannot be neglected because it is uniquely language specific. In this presentation it is discussed how knowledge of representations of within-word structure plays a significant role in recognition of morae from the point of psycholinguistic framework, presenting various types of data that were collected from (1) Japanese preschool children (before and after acquiring kana orthography), (2) Japanese monolinguals and Japanese English bilinguals, and (3) Japanese L2 learners (from beginning to advanced whose native language is English). Also reported are data from Telugu (a Dravidian language spoken in India), whose speech segmentation mechanism behaves like that of mora-timed language. It is hoped that knowledge of representations of within-word structure together with the durational properties will facilitate Japanese learners to acquire Japanese speech timing.

$$
\text { 3:25-3:55 Break }
$$

3pSC8. DiscourseWare, a blended learning courseware for understanding and producing Japanese audio-visual reactive tokens. Goh Kawai and Takafumi Utashiro (Inst. of Lang. and Culture Studies, Hokkaido Univ., Sapporo, Hokkaido 060-0817 Japan, goh@kawai.com)

We developed blended learning courseware called DiscourseWare for learning Japanese audio-visual reactive tokens. Acquiring proper token choice, timing, frequency, duration, and intonation are emphasized. The courseware consists of interactive computerbased multimedia material and classroom instruction. Lessons start with instructor-led classroom instruction on the types and usage of various reactive tokens. Learners then independently use interactive computer-based courseware to learn how to choose appropriate reactive tokens. The courseware contains videos showing two native speakers conversing using a uncontrolled vocabulary and syntax and at a natural speed. The reactive token being taught is blanked out. Learners solve multiple-choice problems asking which reactive token would be appropriate in that context. Learners increase proficiency in interpreting various prosodic patterns of the same phonetic string. After computer-based training, learners regroup and practice exchanging reactive tokens. Our courseware is accompanied by a syllabus for reactive tokens that might interest researchers of comparative pragmatics as well as language instructors. The conference presentation will include DiscourseWare demonstrations, and before-and-after training comparisons of non-native learners' reactive token productions as assessed through interviews with language teachers.

\section{4:15}

3pSC9. An issue of rate normalization for learners of Japanese. Yukari Hirata (Dept. of East Asian Lang. and Lit., Colgate Univ., 13 Oak Dr., Hamilton, NY 13346)

This talk explores native and non-native speakers' identification of Japanese phonemic length contrasts in sentences of varying speaking rates. Native Japanese speakers were found to use the rate of the sentence surrounding a target word as a perceptual cue (i.e., to normalize speech rate) to distinguish phonemic vowel and consonant length. This perceptual cue overrode more local cues such as physical duration of the target vowel or consonant. However, when beginning learners of Japanese participated in the same listening tasks, they were unable to normalize speech rate and identify length of vowels and consonants as native speakers did. Specifically, when asked to identify the length of vowels in $/ \mathrm{mV}(\mathrm{V}) \mathrm{mV}(\mathrm{V}) /$ (with the first vowel accented) in sentences of slow and fast rates, the learners were least accurate in identifying the long vowel of $/ \mathrm{mVmVV} /$ in sentences spoken at a fast rate. The learners' perception was biased by physical duration of vowels and by the presence or absence of pitch accent. Their identification accuracy for long vowels was more affected by speaking rate than that for short vowels. Taken together, these results suggest a need for a training program to help learners of Japanese to cope with speaking rate variations.

\section{$4: 35$}

3pSC10. Effects of exposure and training on perception of Japanese length contrasts by English listeners. Keiichi Tajima (Dept. of Psych., Hosei Univ., 2-17-1 Fujimi, Chiyoda-ku, Tokyo 102-8160, Japan, tajima@i.hosei.ac.jp), Hiroaki Kato (NICT/ATR, Kyoto 619-0288, Japan), Amanda Rothwell, Reiko Akahane-Yamada (ATR, Kyoto 619-0288, Japan), and Kevin G. Munhall (Queen's Univ., Kingston, ON, Canada)

Native English listeners are known to have difficulty distinguishing Japanese words that contrast in phonemic length, often realized as a contrast in vowel or consonant duration. The present study reports results from a series of experiments investigating the extent to which English listeners' perception of such length contrasts can be modified with exposure to Japanese and with perceptual identification training. Listeners were trained in a minimal-pair identification paradigm with feedback. A pretest and posttest were also administered, using natural tokens of Japanese words containing various vowel and consonant length contrasts, produced in isolation and in a carrier sentence, at three speaking rates, and by multiple talkers. Results indicated that exposure and perceptual training substantially improved identification accuracy. Even though listeners were trained to identify words contrasting in vowel length only, performance also improved for other contrast types. Furthermore, speaking rate strongly affected performance, but training improved performance at all three rates, even though listeners were trained using only stimuli spoken at a normal rate. These results suggest that non-native listeners are highly susceptible to factors that affect the temporal characteristics of speech, but their perceptual strategies can be modified with exposure and training. [Work supported by NICT and JSPS.] 
3pSC11. Categorization of phonemic length contrasts in Japanese by native and non-native listeners. Hiroaki Kato (NICT/ ATR, 2-2-2 Hikaridai, Seika-cho, Soraku-gun, Kyoto 619-0288, Japan, kato@atr.jp), Keiichi Tajima (Hosei Univ.), Amanda Rothwell, Reiko Akahane-Yamada (ATR), and Kevin Munhall (Queen's Univ.)

Previous studies reported that exposure to Japanese and identification training allowed English listeners to improve their identification accuracy of Japanese words with phonemic length contrasts. However, this does not necessarily mean their perceptual properties approach those of native listeners in all respects. Past perceptual studies have shown that native listeners show, for example, an extremely sharp short-to-long boundary and shift of the boundary to adapt to changes in the temporal context, i.e., typically speaking rate variations. To carefully investigate the effects of training on such properties, the present study analyzed the learners' identification of stimulus continua between word pairs that minimally differed in the length of a phoneme. Overall results showed that English listeners' boundaries tended to be sharpened by the identification training, but only to a limited extent. On the other hand, the results also showed that English listeners' boundaries shifted across different speaking rates in ways that resemble native listeners' adaptation tendencies. The latter result suggests that even non-native listeners can adjust their identification boundaries according to differences in temporal context. Discussion will include both prospects and limitations of listeners' improvement by the perceptual training in this study. [Work supported by NICT and JSPS.]

THURSDAY AFTERNOON, 30 NOVEMBER 2006

MOLOKAI ROOM, 1:00 TO 5:00 P.M.

\title{
Session 3pSP
}

\section{Signal Processing in Acoustics: Various Topics in Signal Processing (Poster Session)}

\author{
Charles F. Gaumond, Cochair \\ Naval Research Lab., Code 7142, 4555 Overlook Ave., S.W., Washington, DC 20375-5320 \\ Yoshinobu Kajikawa, Cochair \\ Kansai Univ., Dept. of Electronics, Faculty of Engineering, 3-3-35 Yamate-cho, Suita-shi, Osaka 564-8680, Japan
}

\section{Contributed Papers}

\begin{abstract}
All posters will be on display from 1:00 p.m. to 5:00 p.m. To allow contributors an opportunity to see other posters, contributors of odd-numbered papers will be at their posters from 1:00 p.m. to 3:00 p.m. and contributors of even-numbered papers will be at their posters from 3:00 p.m. to 5:00 p.m.
\end{abstract}

3pSP1. Adaptive surround technology for stereo music signals. Yoshitaka Murayama, Akira Gotoh, Jun Ota, Hareo Hamada (DiMAGIC, Sugisho Bldg 3F, 2-4-5 Kanda-Ogawamachi, Chiyodaku, Tokyo 101-0052, Japan), Yuko Watanabe, and Hareo Hamada (Tokyo Denki Univ., Inzai-shi, Chiba, Japan)

Computation of the left and right channel instantaneous difference ( $\mathrm{xL}-\mathrm{xR}$ ) is mostly used to obtain the signals for surround sound. This signal is usually referred as the antiphase components. However, there are many problems to using the antiphase components in terms of its applicability in practice as well as the sound quality. For instance, when signals $\mathrm{xL}$ and $\mathrm{xR}$ are equal, or almost equal, this variation, that is to say, the correlation coefficient value, is high; there is no way to determine the direction vector uniquely, therefore an ambiguity may appear. Instead of the antiphase component's computation, a new method is proposed by using an adaptive signal processing technique. In this paper, it is revealed that natural surround sound signals having no antiphase and uncomfortable feeling are adaptively generated by the proposed method. The other advantages from a signal processing point of view are also discussed. Finally, subjective evaluations on sound quality of a surround sound system are carried out.
3pSP2. A design of fast steering filters based on the adaptive fusion of predesigned finite impulse response filters for microphone array. Masato Nakayama (Grad. School of Sci. and Eng., Ritsumeikan Univ., 1-1-1 Noji Higashi, Kusatsu, Shiga 525-8577, Japan, gr020040@se.ritsumei.ac.jp), Takanobu Nishiura, and Yoichi Yamashita (Ritsumeikan Univ., Kusatsu, Shiga 525-8577, Japan)

With a microphone array, a desired distant-talking speech signal can be acquired selectively by steering the directivity in noisy environments. To form directivity, adaptive beamformers have been proposed as conventional beamformers. They can form null directivity with a small number of transducers. However, adaptive beamformers have a serious drawback: it can't realize the noise-robust adaptation in variable noisy environments, because it has necessary heavy computational costs to train multi-channel adaptive steering filters. To solve this problem, we propose to design the fast steering filters based on the adaptive fusion of predesigned FIR filters in variable noisy environments. The adaptive fusion of predesigned FIR filters is realized by adding (or subtracting) various predesigned FIR filters immediately. Therefore, the proposed method can quickly design the steering filters even in variable noisy environments. In addition, microphone calibration is indispensable for applying the proposed method. Therefore, we also propose the new microphone calibration method based on environmental noise without target signal. Evaluation experiments were con- 
ducted in a real acoustic room. As a result of evaluation experiments, we could confirm that the ASR (automatic speech recognition) performance of distant-talking speech was improved on the well-known condition of noise source directions. [Work supported by MEXT of Japan.]

3pSP3. A frequency domain method to estimate the coefficients of feedback control filter. Yusuke Iwamatsu, Masahiro Itou, and Kensaku Fujii (Div. of Comp. Eng., Univ. of Hyogo, 2167 Shosha, Himeji, 671-2280, Japan,er05i005@ steng.u-hyogo.ac.jp)

This study proposes a method for repeatedly updating coefficients of a feedback control filter used for canceling a feedback path from a loudspeaker to a noise detection microphone. The coefficients are typically estimated by feeding a sequence of extra noise to the loudspeaker. However, feeding such extra noise under active noise control is undesirable. The proposed method estimates the coefficients without feeding the extra noise. Using the estimation errors involved in the coefficients of the noise control filter instead of the extra noise, the proposed method provides two independent equations and estimates coefficients of the feedback control filter by solving those. The two independent equations are provided through linear prediction using an auxiliary filter. A solution of the equations with two unknowns (a primary noise and the feedback path) provides the coefficients of the feedback control filter, perfectly canceling the feedback path. A salient drawback is that the feedback component is extremely small in comparison to the primary noise that is incident on the noise detection microphone. For that reason, estimation of the coefficients is difficult. This method solves the problem by first estimating the primary noise and then the feedback path.

3pSP4. A new multichannel adaptive algorithm. Masahiro Itoh, Kensaku Fujii (Div. of Comput. Eng., Univ. of Hyogo, 2167 Shosha, Himeji, 671-2280 Japan, er05a003@steng.u-hyogo.ac.jp), and Mitsuji Muneyasu (Kansai Univ., 3-3-35 Yamate-chou, 564-8680 Suita, Japan)

This study presents a new multichannel adaptive algorithm that identifies unknown systems without superposing independent components on reference signals. A problem in such multichannel systems is that the reference signals involve strong cross-correlative components between themselves. These components delay the convergence of the coefficients of adaptive filters. At worst, they can render identification impossible. Usually, this problem is solved by superposing slightly independent components on the reference signals. Various superposition methods are therefore proposed. However, this superposition is perceivable as discordant noise. This study hence examines a new algorithm that exploits the residual components obtained by applying a linear prediction technique to the reference signals. The cross-correlative components thereby decrease and the convergence is hastened. Using this algorithm, however, the residual components are not fed into the unknown systems and adaptive filters. This is a point of great difference from conventional methods. This study also analyzes the performance of the proposed algorithm and derives the convergence condition as a range of step size. Finally, computer simulations are used to verify the derived convergence condition and the increased convergence speed.

3pSP5. Frequency domain simultaneous equations method for active noise control systems. Tadashi Ujino, Kensaku Fujii (Div. of Comput. Eng., Univ. of Hyogo, 2167 Shosha, Himeji, 671-2280 Japan, er06e006@steng.u-hyogo.ac.jp), Mitsuji Muneyasu (Kansai Univ., 564-8680, Suita, Japan), and Yusuke Fujita (Catsystem Corp., 671-1121, Himeji, Japan)

This study proposes a new method for feedforward-type active noise control systems. This method, named the frequency domain simultaneous equations method, is based on a different principle from the filtered- $x$ algorithm requiring a filter modeled on a secondary path from a loudspeaker to an error microphone. Instead of the filter, this method uses an auxiliary filter identifying the overall path consisting of a primary path, a noise control filter, and the secondary path. As seen from the configuration of the overall path, the auxiliary filter can provide two independent equations when two different coefficient vectors are given to the noise control filter. In practical use, the two different coefficient vectors are provided by their own estimation errors. By solving the independent equations, the proposed method can estimate the coefficient vector of the noise control filter minimizing the output of the error microphone. This study first presents computer simulation results demonstrating that the proposed method can automatically recover the noise reduction that is in effect degraded by path changes. Finally, this study applies the proposed method to an experimental system and, by using the system, verifies that the proposed method is functional in practical systems.

3pSP6. Partial differential equation (PDE)-based method of sound source localization. Shigeru Ando and Nobutaka Ono (Univ. of Tokyo, 7-3-1 Hongo, Bunkyo-ku, Tokyo 113-8656 Japan, ando@alab.t.u-tokyo.ac.jp)

We describe the problem of direction and distance estimate of sound sources in free 3-D space. We first derive a partial differential equation (PDE), $\nabla f(\mathbf{r}, t)=\{(1 / R) f(\mathbf{r}, t)+(1 / c) \dot{f}(\mathbf{r}, t)\} \mathbf{n}(R \equiv|\mathbf{r}|, \mathbf{n} \equiv-\mathbf{r} / R)$, what we call the sound source constraint (SSC). We show that the general solution of the SSC is a diverging spherical wave from a point sounce with arbitrary temporal waveform. The SSC enables the observer to determine the source location (distance $R$ and direction $\mathbf{n}$ ) from local measurements of the wave field. As the measurements of the wave field, we consider two methods: (1) the weighted temporal integral of arrayed microphone outputs in a finite duration and (2) the weighted spatial integral of pressure distribution in a rectangular or circular area. In both cases, we obtain exact formulas for localizing a single source from a single measurement and multiple sources from the combination of differently weighted measurements.

3pSP7. Effects of microphone and loudspeaker directivity on accuracy of synthesized wave fronts in sound field reproduction based on wave field synthesis. Toshiyuki Kimura, Sadanori Ito, Masaki Nakagawa (Dept. of Comp. and Info. Sci., Tokyo Univ. of Agriculture and Technol., 2-24-16 Naka-cho, Koganei-shi, Tokyo, 184-8588 Japan), and Kazuhiko Kakehi (Chukyo Univ., Kaizu-cho, Toyota-shi, Aichi, 470-0393 Japan)

Sound field reproduction based on wave field synthesis proposed by Camras [M. Camras, J. Acoust. Soc. Am. 43, 1425-1431 (1968)] is a technique that synthesizes wave fronts of a control area at a listening area using directional microphones and loudspeakers placed at the boundary of those areas. This study evaluates the effects of the directivity of microphones and loudspeakers on the accuracy of synthesized wave fronts. Microphones and loudspeakers were placed at the boundary of both areas that have two cases (a circle with a radius of $2 \mathrm{~m}$ and a square $4 \mathrm{~m}$ on a side). The number of microphones and loudspeakers were 630 (circle) and 801 (square). The interval of microphones and loudspeakers was less than 2 $\mathrm{cm}$. Therefore, spatial aliasing does not occur if the frequency is less than $8 \mathrm{kHz}$. Three directional patterns (omnidirectional, unidirectional, and shotgun) were designed as directivity conditions of microphones and loudspeakers. Computer simulation results showed that there is no effect of directivity of loudspeakers and that accurate wave fronts can be reproduced when a unidirectional or shotgun microphone is applied. [Work supported by special grant for education and research of Tokyo Univ. of Agriculture and Technol.] 
3pSP8. A study of pinna effect on head-related transfer functions. Hiromi Sekido, Yuko Watanabe, and Hareo Hamada (Tokyo Denki Univ., 2-1200 Muzai-gakuendai, Inzai, Chiba Prefecture 270-1382, Japan)

For the sound localization, an interaural difference is important. To obtain the desired localization, it is necessary to use a head-related transfer function (refer to HRTF), which is defined as the transfer function between a sound source and a surface of the ear drum. In our previous work, as far as in the case of reproducing the virtual sound source in the horizontal plane, it is confirmed to be capable of remarkable localization by using the numeric data calculated by a rigid sphere head model instead of a dummy head microphone. However, it is necessary to solve the problem of the front back confusion and the sound localization of the vertical plane. For that, it might be essential to add the pinna information to the rigid sphere model, to examine how each part of the pinna is related to the localization.

3pSP9. Sound reproduction system with switching control band range on every listening point. Yosuke Tatekura and Takeshi Watanabe (Dept. of Eng., Shizuoka Univ., 3-5-1 Johoku, Hamamatsu, Shizuoka, 432-8561, Japan)

We propose a robust multichannel sound reproduction system that utilizes the relationship between the width of the actual listening area and the control frequency of the listening points. The conventional multichannel sound reproduction system uses inverse filters that are designed based on room transfer functions (RTFs) from loudspeakers to listening points in the listening room. However, the sound reproduction accuracy is deteriorated by room environment variations when fixed inverse filter coefficients are used because the condition number relevant to the instability of the matrix composed of the RTFs is large, especially at low frequencies; the inverse filters are unstable at those frequencies. This tendency is more remarkable when listening points are arranged more closely. To resolve the problem, we switch the control band at every listening point to avoid degrading the reproduced sound in low-band frequencies. The control band range of the listening points at both ears is only high band. That of the other listening points is the entire control band. Numerical simulation with real environmental data showed that improvement of the reproduction accuracy is about $6.4 \mathrm{~dB}$ on average, even with temperature fluctuations of $5{ }^{\circ} \mathrm{C}$ as environmental variations of the listening room.

3pSP10. Frequency scaling reduces individual differences in externalear transfer functions in developing cats. Daniel J. Tollin (Dept. Physiol \& Biophys., Univ. Colorado Med. Sci., 12800 E. 19th Ave., Aurora, CO 80045, daniel.tollin@uchsc.edu)

There are three cues to sound location: Interaural differences in time and level and monaural spectral shape cues. The ultimate magnitudes of these cues depend largely on the linear dimensions of head and pinnae. During development, the increasing interaural distance associated with a growing head and pinnae result in cues that change substantially and continuously until maturation is complete. These experiments examined whether the intersubject differences in the directional transfer functions (DTFs), the directional components of the head-related transfer functions, of cats during development could be reduced by scaling one set of DTFs on a log-frequency axis. Measurements were made of both the physical dimensions of the head and pinnae and the DTFs of cats aged 4 weeks to adulthood. For each pair of subjects, an optimal frequency scaling factor was computed that minimized the differences in the DTFs. In many cases, the optimal factors for pairs of subjects were proportional to the ratios of the measured physical dimensions of the head and pinnae in those subjects. Although there were still considerable differences in the DTFs even after scaling, these data support frequency scaling as a potential method of modeling the development of the DTFs in cats. [Work supported by NIH Grant DC006865.]

3pSP11. A method to make a moving virtual sound source. Ji-Ho Chang and Yang-Hann Kim (KAIST (Korea Adv. Inst. of Sci. and Technol.), 373-1 Kusong-dong, Yusong-gu, Daejeon 305-701, Korea)

This study deals with a method that manipulates the sound field, which is excited by a moving source. That is, the moving source is virtually constructed by array speakers. It is realized by controlling pressure values on boundary of the field. This is, in fact, an attempt to implement the Kirchhoff-Helmholtz integral equation in practice. It is well known that we can generate a certain sound field in a space by controlling pressure values on boundary [S. Ise, Acustica, 85, 78-87 (1999)]. However, time varying sound field control has not been well addressed. Our aim is to manipulate sound field that is generated by a virtual source moving straightly outside the sound field or traveling around it. To express the method, the simple pure-tone case is considered and then expanded to more general cases. It also handled the problem related to eigenfrequencies at which boundary pressure control is not applicable. Computational and experimental results are introduced as well. [Work supported by BK21 \& NRL.]

3pSP12. Partial-differential-equation-based sound source localization: Finite Fourier integral approach and its application to multiple source localization. Yuya Fujita, Nobutaka Ono, and Shigeru Ando (Dept. of Information Phys. and Computing, Grad. School of Information Sci. and Technol., The Univ. of Tokyo, Tokyo, Japan)

In this paper, a method based on the sound source constraint PDE (partial differential equation) is developed for real complex environments with multiple sources. The sound source constraint PDE is integrated within a finite temporal interval with orthogonal Fourier bases to obtain simultaneous equations including source directions and distances as unknown variables. In them, the fast Fourier transform can be used rigorously and efficiently with least computation time. We show the equations enable us to obtain 3-D locations of up to four sources simultaneously from one spatial gradient observation of sound field. Numerical simulation comparison with the spatio-temporal-gradient-based method was carried out and showed the significant improvement of accuracy. Experimental results using a six proximity microphone array [N. Ono et al., in Proc. ASJ Autumn meetingCvol.IC, pp. 607-608, September, 2004 (in Japanese)] in the real environment will be reported.

3pSP13. Holographic diagnosis: Basic principles and applications. Jong-Hoon Jeon and Yang-Hann Kim (Ctr. for NOVIC, Dept. of Mech. Eng., KAIST, 373-1 Guseong-dong, Yuseong-gu, Daejeon 305-701, Republic of Korea)

Acoustic signals achieved from a machine often have information on condition of the machine. This means that the location of faults can be found by using acoustic signal. Location of sound source can be found by using techniques such as MFAH (moving frame acoustic holography), which is applicable for pure tone noise and good signal-to-noise ratio, and beamforming, which basically assumes no reflected wave. Faults due to breakdown of rotating parts in a moving vehicle, however, make the above method hard to be directly applicable. The difficulty stems from three characteristics in the fault detection of the moving vehicle. First, the sound source radiates periodic impulsive noise. Second, there exist reflected waves from the ground since the array microphones should be settled between the vehicle and the ground to monitor the bottom side of the vehicle. Last, for the measurement problem, there exists noise due to other parts and wind for the moving vehicle, which degrades coherence among measured signals. In this research, holographic diagnosis, a machine diag- 
nosis technique based on holographic approach, is introduced to find the position of faults effectively. The modified version of beamforming that models the moving periodic impulsive noise as a scan function is introduced.

3pSP14. Software-centered implementation of 128-channel loudspeaker array with stock PC and its application. Hiroshi Mizoguchi, Takafumi Nakamura, Hiroshi Takemura (Tokyo Univ. of Sci., 2641 Noda, Chiba 278-8510, Japan), and Satoshi Kagami (DHRC, AIST, Japan)

A huge speaker array system of 128 loudspeakers was constructed and experimented. It was implemented as "software-centered" style, utilizing stock loudspeakers and a PC. No dedicated hardware nor DSP was utilized. Spot forming, instead of beam forming, could be realized by 32 by 4 square layout of the array. Spot means small area of higher sound pressure level. Number of the spot was not limited to one. In the experiment, within $3 \mathrm{~m}$ by $3 \mathrm{~m}$ area, four spots of different sounds could be simultaneously formed. This spot forming was confirmed by actually measuring spatial distribution of sound pressure level. The effect of the spot was also confirmed auditorily. Since the system was software centered, it was dynamic. By simply changing software parameters, the location of the spot can be easily moved even while the system is running. This movability of the spot was intended to be the basis for visual steering. To realize the system, a simultaneous 128-channel 14-bit DA converter PCI board was developed. The 44.1-KHz sampling rate was achieved by a 2.4-GHz Intel Xeon based PC utilizing the DA board and a real-time OS, named ARTLinux. Approximately $23-\mu$ s loop could be realized by the software. It was the world's fastest software loop.

3pSP15. Complex envelope implementation for fast and efficient acoustic holography. Choon-Su Park and Yang-Hann Kim (Ctr. for Noise and Vib. Control, Dept. of Mech. Eng., KAIST, Daejeon 350-701, Republic of Korea)

Acoustic holography allows us to predict the spatial pressure distribution on any surface of interest. To implement this method, we normally use Fourier transform in time and space domain. It is noteworthy that the data size is so huge that it takes a long time to calculate pressure field. Moreover, the reconstructed pressure field is frequently too complex to observe the characteristics of pressure field. One possible candidate is the complex envelope. The complex envelope in time domain is well known and widely used in various fields. We have attempted to extend this method in the space domain, so that we can have a rather compact sound pressure picture that provides the information we need, for example where sound sources are. This belongs to what we call the analysis problem of acoustic holography. We might want to draw some parts in detail but other parts in a rather coarse way. The complex envelope in space certainly meets this objective. First we start with the simplest case. We devise a monopole complex envelope. Then we extend it to finite size source case. Various holography examples are reprocessed according to what we propose and demonstrate how this method is practically fast and how it provides a better picture for analyzing the sound field.

3pSP16. The acoustic information extraction of the indoor impulse response by multiresolution analysis. Wakako Tanaka, Yong Tang, Toru Itakura, and Hideo Shibayama (3-7-5 Toyosu Koutou-ku, Tokyo, 135-8548, Japan,m105072@sic.shibaura-it.ac.jp)

The impulse response is divided into approximation and detail components by the multiresolution analysis. The correlations between the early sound and reflected sounds are estimated respectively for the impulse response and the approximate signal. The approximate signal was calculated as a function of level of the multiresolution analysis. The correlation coefficient of these correlations is over 0.7 under level three. We evaluate the differences of these correlations from the listening test.
3pSP17. Detection for the early reflection in the impulse response by the correlation function. Toru Itakura, Yong Tang, Wakako Tanaka, and Hideo Shibayama (Shibaura Inst. of Technol., Toyosu 3-7-5, Koutoku-ku, Tokyo 135-0061, Japan,m106009@sic.shibaura-it.ac.jp)

Detecting an early reflection sound contained in the impulse response measured in a room, we calculated the correlation factor between the early reflection and the impulse response. Calculating the correlation factor every sampling period, we make the correlation function. For the function, we set a threshold level. We detected the absolute correlation value larger than the threshold level. And, we set the zero value for subthreshold level. Following this algorithm, we generate a new signal with the high correlation factor for the early reflection. We compared the signal with the impulse response by using listening test.

3pSP18. A design of virtual sound field simulator based on acoustic ray tracing and wall impedance. Kiichiro Yamada, Yuki Denda, Masato Nakayama (Grad. School of Sci. and Eng., Ritsumeikan Univ., 1-1-1 Noji Higashi, Kusatsu, Shiga 525-8577, Japan, rs047023@se.ritsumei.ac.jp), and Takanobu Nishiura (Ritsumeikan Univ., Kusatsu, Shiga 525-8577, Japan)

The sound propagation analysis using an architectural scale model is an effective approach for architectural acoustic design of a concert hall, and so on. However, the scale model must be reconstructed together, changing the basic plan of the architectural design. To cope with this problem, an architectural sound field has been virtually designed on the computer by the sound field simulator in recent years. The acoustic ray tracing is one of the simulators based on geometric acoustics. It is an efficient solution from the viewpoints of simulation cost and time. However, it cannot simulate the wall materials, because it employs the delta function as the wall impedance. To overcome this problem, in this paper, we try to design the virtual sound field simulator based on acoustic ray tracing and wall impedance acquired by real measurements. We first measured the reflection characteristics of the wall with impulse response to acquire the wall impedance. Then, we designed the virtual sound field simulator by convoluting the acoustic rays with measured wall impedances. As a result of subjective evaluation experiments, the proposed simulator could realize a more realistic sound field than the conventional acoustic ray tracing. [Work supported by MEXT of Japan.]

3pSP19. A fundamental investigation of the small microphone array system and its applications. Akira Nawa, Yoku Watanabe, and Hareo Hamada (Tokyo Denki Univ., 2-1200 Muzai-gakuendai, Inzai, Choba prefecture 270-1382, Japan)

A purpose of this research is to construct a 3-D sound recording and reproduction system that has a high presence by performing a spatially controlled sound field synthesis technique. For this purpose, it will be required to examine and choose the most suitable sound recording system, since a conventional sound recording system samples only original signal itself, and does not include enough spatial information. In other words, it will be required to examine the system to record the information of the three-dimensional spaces, such as the direction and the distance, and to transform the configuration to become effective to the reproduction system. This paper will deal with a basic examination to construct the microphone array placed in proximity and the recording system, which is able to control the directivities in the three-dimensional spaces, using algorithm based on LMS by FIR filter networks from signals to each microphone. 
3pSP20. Blind source separation of musical signals applied to selectable-listening-point audio reconstruction. Kenta Niwa (Grad. School of Information Sci., Nagoya Univ., Furo-cho, Chikusa-ku, Nagoya-city, Aichi, Japan, niwa@sp.m.is.nagoya-u.ac.jp), Takanori Nishino, Chiyomi Miyajima, and Kazuya Takeda (Nagoya Univ., Japan)

An audio reproduction system is being developed to generate binaural signals at user-selected listening positions from audio signals of multiple sound sources recorded with multiple microphones. The system consists of a sound decomposer based on blind source separation (BSS) and a sound recomposer using head-related transfer functions (HRTFs). In this work, musical signals of multiple instruments are separated into the original independent signals of the respective instruments using BSS based on frequency-domain independent component analysis (FD-ICA). HRTFs are then convoluted to the separated sound sources to reconstruct the music sounds of the selected listening point. Preliminary experiments are conducted using two or three sound sources of instruments: flute, violin, and piano generated by a synthesizer. The synthesizer sounds are played through small directional loudspeakers or spherical omnidirectional loudspeakers in a low-reverberant sound-proof room or moderately reverberant lecture room and recorded with multiple microphones. Live music played on real instruments including flute, violin, and trumpet are also recorded in the same rooms. Audio signals of respective instruments obtained by BSS are available on our web site.

3pSP21. Acoustic sound field simulator based on head-related transfer function and three-dimensional mirror-image. Keitaro Kuroda, Yuki Denda, Masato Nakayama (Grad. School of Sci. and Eng., Ritsumeikan Univ., 1-1-1 Noji Higashi, Kusatsu, Shiga 525-8577, Japan, rs016029@se.ritsumei.ac.jp), and Takanobu Nishiura (Ritsumeikan Univ., Kusatsu, Shiga 525-8577, Japan)

The head-related transfer function (HRTF) is the most important factor to simulate an acoustic sound field for the virtual reality technology or the architecture field. However, the HRTF has individual differences on every listener, because it represents the real transfer function between the sound source point in the acoustic field and the listener's ears. In addition, it is impossible to measure the HRTFs on every listener. To solve this problem, we focus on three-dimensional mirror-image. It can represent the virtual transfer function (especially, room reverberation) in an acoustic field by replacing all surface reflections with mirror-image sources. Therefore, we try to design the new acoustic sound field simulator by convoluting the real transfer function of the HRTF and the virtual transfer functions of the three-dimensional mirror-image. By this approach, the proposed simulator may realize the more accurate sound-image localization with room reverberation by three-dimensional mirror-image even if the HRTF of an other listener is employed. As a result of evaluation experiments in some conditions, the proposed simulator could improve the sound-image localization performance especially in an anteroposterior direction more than the conventional simulator based on HRTF. [Work supported by MEXT of Japan.]

3pSP22. Binaural transformation coding with simulated head tracking. Seiichiro Shoji and Anthony I. Tew (Dept. of Electron., Univ. of York, Heslington, York, YO10 5DD UK)

The binaural transformation codec synthesizes generic binaural audio signals and generates accompanying side information. In the decoder the side information is used to personalize the generic signal. The performance of the basic codec is described in another paper; this paper describes the estimated effect on quality of incorporating limited head tracking. Generic HRTFs were used to spatialize two concurrent sound sources spatialized at \pm 30 or $\pm 80 \mathrm{deg}$. The generic binaural signal was personalized in the BX decoder and at the same time the individual sound sources were rotated. These induced rotations are equivalent to compensating for head yaw rotations of $-80,-40,-20,-10$, and $-5 \mathrm{deg}$, and for head pitch rotations of $22.5,45$, and $90 \mathrm{deg}$. Listening tests based on Recommendation ITU-R BS.1116-1 were used to evaluate the processed sounds. The tests were conducted using speech, vocals, guitars, and percussion source materials. It was found that the quality of the processed sound tended to degrade as the head yaw rotation increased. However, except for the head yaw condition $-80 \mathrm{deg}$, the quality of the processed sound remained greater than 4.0 on the mean opinion scale. No deterioration of the processed sound was observed for the applied pitch rotations.

3pSP23. Novel method for the efficient individualization of binaural audio signals. Seiichiro Shoji and Anthony I. Tew (Electron. Dept., Univ. of York, Heslington, York, YO10 5DD UK)

A novel coding technique suitable for the transmission of binaural audio signals with concurrent multiple sound sources is proposed in this paper. Existing algorithms typically suffer from audible artefacts, poor localization, or excessive bandwidth. Especially with conventional intrasource coding methods, bandwidth is dependent on the number of sources and becomes greater as the number of sources increases. The proposed binaural transformation (BX) encoder synthesizes binaural signals using generic HRTFs and transmits them to the decoder along with side information. The bandwidth of the side information is independent of the number of sources. Using locally stored HRTFs matching those of the listener, the decoder turns the generic binaural signals into personalized ones. We present two types of BX codec: type A and type B. Psychoacoustic experiments based on Recommendation ITU-R BS.1116-1 were used to compare signals processed by these codecs with the corresponding unprocessed signals. For a variety of source materials (speech, vocals, guitars, and percussion) we show that high mean opinion scores are achievable using the type A codec when its parameters are set appropriately.

3pSP24. Individuality of elevation-angle-sensitive notches in headrelated transfer functions. Saho Sekimoto, Yukio Iwaya, and Yôiti Suzuki (Res. Inst. of Elec. Commun./Grad.. School of Information Sci., Tohoku Univ. Katahira 2-1-1 Aoba-ku, Sendai, 980-8577, Japan, saho@ais.riec.tohoku.ac.jp)

Perceived localization can be controlled with high accuracy in the rendered auditory space when listeners' own head-related transfer functions (HRTFs) are used for rendering 3-D spatial audio. However, measurement of a listener's own HRTFs in all directions requires a special measuring apparatus and a long measurement time, which entail a considerable physical load on subjects. Therefore, effective individualization methods of HRTFs must be very useful. In previous studies, a few notches around $6-10 \mathrm{kHz}$, whose frequencies change depending on the elevation, are inferred as important to determine the perceived elevation [e.g., E. A. G. Shaw and R. Teranishi, J. Acoust. Soc. Am. 44, 240-249 (1968)]. Therefore, this study examines the individuality of such notches, of which the frequency is elevation-angle sensitive. First, individual HRTFs from all directions are measured for 140 listeners. They are then analyzed using an autoregressive moving average (ARMA) model. Second, the frequencies of these notches are expressed smoothly as a function of the elevation angle using dynamic programming (DP). Results qualitatively clarified the dependency of each frequency on elevation. Moreover, distributions of the notch frequencies show systematic variation as the elevation angle changes.

3pSP25. Objective and subjective evaluation of numerically estimated head-related transfer functions. Shouichi Takane and Koji Abe (Dept. of Electron. and Information Systems, Akita PRef. Univ., 84-4 Ebinokuchi, Tsuchiya, Yurihonjo, Akita, 015-0055 Japan, takane@akita-pu.ac.jp)

In this study, head-related transfer functions (HRTFs) of a dummy head and human subjects are numerically estimated by using the boundary element method (BEM), and their availability for auralization is discussed from the subjective viewpoints. Geometries of their heads are obtained by a 3-D laser scanner. The effect of the torso for the estimation of the HRTFs is also examined and discussed via the 3-D measurement. The estimated 
HRTFs are compared for each subject with the measured HRTFs in various objective criteria, such as spectral distortion (SD), signal-to-distortion ratio (SDR), and interaural time/level differences (ITD/ILD). Availability of the estimated HRTFs for the synthesis of the 3-D sound image are then evaluated via hearing experiments. In our previous study [Proceedings of WESPAC IX (2006)], the results of the experiment is insufficient since the low-frequency range of the estimated HRTFs caused the ambiguity in sound localization. In this study, the frequency range of the estimation is extended to as high frequency as possible, and the availability of the estimated HRTFs for auralization is discussed from two viewpoints: the subjective difference between the estimated and measured HRTFs, and some subjective attributes obtained from the hearing of the stimuli convoluted with the estimated HRTFs.

3pSP26. Analysis and modeling of interaction of auditory-visual information. Takahumi Asada, Yuko Watanabe, and Tatsuya Shibata (Grad. School of Information Environment, Tokyo Denki Univ., 2-1200 Muzai Gakuendai Inzai, Chiba, 270-1382 Japan, den03117@nifty.com)

We analyze and model the interaction of movement of the synchronized auditory-visual information. We construct the relationship of movement between virtually sound source and object. We suppose that cubic local contrast model (CLCM) we propose and the optical flow method are useful for analyzing moving image. CLCM is a method that describes the movement of objects from temporal and spatial difference of local contrast between pixels in moving image, and we found CLCM can measure the orientation of camera motion and the moving direction of an object in a moving image. We use the feature of head-related transfer function (HRTF) as the feature of sound movement. We have done two experiments: experiment 1 is that we analyze the data of the interaural amplitude difference (IAD) and interaural phase difference (IPD) between right and left HRTFs of a sphere by local contrast model (LCM); experiment 2 is that we analyze the relationship between the movement of an object in a moving image and a virtual sound source. We discuss the interactive features between auditory and visual information.

3pSP27. The effect of head movement on sound localization using stereo microphones: Comparison with dummy heads. Wataru Endo (Tokyo Inst. Tech., Nagatsuda, Midori-ku, Yokohama 226-8502, Japan, endo@u.ip.titech.ac.jp), Makio Kashino (Tokyo Inst. Tech., Atsugi, Kanagawa 243-0198, Japan), and Tatsuya Hirahara (Toyama Prefectural Univ., Imizu, Toyama 939-0398, Japan)

This study examined the effect of head movement on sound localization with a pair of microphones providing no head-related transfer function (HRTF) information, and with individualized, nonindividualized, and downsized dummy heads providing HRTF information with different degrees of distortion. In an anechoic room, white noise was presented for 5 $\mathrm{s}$ from one of 12 loudspeakers. A dummy head or a microphone pair was placed at the observation point. Listeners heard the sound via headphones outside the anechoic room and judged the direction of the source and whether the sound image was extracranial. Without head movement, localization accuracy with the microphone pair was significantly worse than that with dummy heads. When voluntary head movement was allowed and the dummy head was moved in accord with the listener's movement, accuracy with nonindividualized and downsized dummy heads improved nearly to the individualized level. Accuracy with the microphone pair also improved with head movement, but did not reach the level of dummy heads. An extracranial image was perceived with dummy heads in most cases, but not often with the microphone pair even with head movement. Head movement cannot completely compensate for the lack of HRTFs.
3pSP28. A biologically inspired pitch determination algorithm. Arturo Camacho and John G. Harris (Computational Neuro-Eng. Lab, Elec. and Comput. Eng., Univ. of Florida, Gainesville, FL 32611, arturo@cnel.ufl.edu)

A biologically inspired pitch determination algorithm is presented. This algorithm combines existing models of the cochlea and inner-haircell based spike generation [Lopez et al., J. Acoust. Soc. Am. 110, 3107 (2001); Sumner et al., ibid. 113, 893-901 (2003)] to model spike trains in the auditory nerve. The pitch and its salience are then estimated using a method proposed by Cariani [Cariani et al., J. Neurophysiol. 76, 16981716], which computes a summary autocorrelation function over the spike trains. Unlike Cariani's work, where spike trains are obtained experimentally, we simulate the spike trains from biologically inspired models. The main contribution of our approach is to combine models of the auditory system and a pitch estimation method based on neural spike trains. The proposed algorithm was tested using standard synthesized sounds, speech, and singing voices. Results show that this algorithm better matches human performance as compared to traditional pitch detection algorithms used in automatic speech processing.

3pSP29. End-point detection of speech using spectral transition for captioning system. Ayako Koga, Yuki Fujikashi, Takayuki Arai (Dept. of Elec. and Electron. Eng., Sophia Univ., 7-1 Kioi-cho, Chiyoda-ku, Tokyo, 102-8554), Noboru Kanedera (Ishikawa Natl. College of Technol.), and Junko Yoshii (Fujiyama, Inc.)

In recent years, captioning video contents with text translations is increasingly necessary because of the burgeoning use of media internationally, resulting from the rapid development of communication technology. In addition, within one language, video captioning is very important for hearing-impaired people. However, the process of captioning videos, including speech and nonspeech decisions, is often done manually by translators at present. Therefore, an efficient automatic end-point detection of speech for captioning video contents has been proposed. We attempted to detect speech end-points based on acoustic landmarks that identify times when acoustic changes are prominent in the speech signals [K. N. Stevens, Acoust. Phonetics (1998)]. In this study, landmarks were obtained by combining the mean square for the regression coefficients of logarithmic envelopes of $\frac{1}{3}$-oct bands in time, which resembles the parameter proposed by Furui to measure spectral transition [S. Furui, J. Acoust. Soc. Am. 80(4), 1016-1025 (1986)], with other ones such as the logarithmic power of speech signals. An experiment was carried out using the proposed technique for speech detection. Results showed a high correct rate and introduced the possibility of its application to an efficient video captioning system. [Work partially supported by Open Research Center Project from MEXT.]

3pSP30. Inverse filter analysis of common harmonic structure on Specmurt using Riemann's $\zeta$ function. Nobutaka Ono, Shoichiro Saito, Hirokazu Kameoka, and Shigeki Sagayama (Dept. Information Phys. and Computing, Grad. School of Information Sci. and Technol., Univ. Tokyo, 7-3-1 Hongo Bunkyo-ku Tokyo, 113-8656 Japan)

In this paper, based on the interesting relationship between the logspaced $\delta$ function sequence and Riemann's $\zeta$ function, the analytical properties of the inverse filter of the common harmonic structure on specmurt analysis are discussed. Specmurt [S. Sagayama et. al., in Proc. SAPA (2004)] is a simple and efficient technique for the multi-pitch analysis of polyphonic music signals. If all tones have the same harmonic pattern, the power spectrum on the log-scaled frequency can be regarded as the convolution of the common harmonic structure and the distribution of fundamental frequencies. Based on the model, overtones are effectively suppressed by the inverse filtering of the common harmonic structure in specmurt. Thus, for the stable processing, analytic properties of the inverse filter are significant. Our new finding is that when the common harmonic structure is expressed as a $\log$-spaced $\delta$ function sequence with a particular kind of decay, the Fourier transform is exactly equal to Riemann's $\zeta$ 
function. Interpreting several properties of Riemann's $\zeta$ function in a signal processing context gives us the new perspectives like an explicit representation or cascaded decomposition of the inverse filter.

3pSP31. The calculation of sleep apnea syndorome's condition index using the snore noise. Takahiko Ono (Tokyo Univ. of Agriculture and Technol., 3-8-1, Harumicho, Fuchu, Japan), HIroo Yano, Tatsuya Oota (Chiba Inst. of Technol., Chiba, Japan), Yuuji Takasaki, and Sachiko Kurosaki (Oota Kinen Hospital, Kooriyama, Japan)

The polysomnograph analysis is used to measure an apnea hypopnea index (AHI) of sleep apnea syndorome. However, this method needs high grade devices and technique. To compensate the conventional method, the calculation of AHI using only the snore noise of the patient is invented.

3pSP32. Voice activity detection with voice/nonvoice classification using reliable fundamental frequency. Soo-young Suk, Hiroaki Kojima (Information Technol. Res. Inst., AIST, Central 2, Umezono, Tsukuba, Japan, sy.suk@aist.go.jp), and Hyun-Yeol Chung (Yeungnam Univ.)

In this paper, we introduce a voice activity detection (VAD) algorithm to perform voice/nonvoice (V/NV) classification using a fundamental frequency $(F 0)$ estimator called YIN. Although current speech recognition technology has achieved high performance, it is insufficient for some applications where high reliability is required, such as voice control of powered-wheelchairs for handicapped persons. The proposed VAD, which rejects nonvoice input in preprocessing, is helpful for realizing a highly reliable system. Previous V/NV classification algorithms have generally adopted statistical analyses of $F 0$, the zero-crossing rate, and the energy of short-time segments. A combination of these methods, a cepstrum-based $F 0$ extractor, has been proposed [S. Ahmadi and S. S. Andreas, IEEE Trans. SAP. 7, 333-339 (1999)]. The proposed V/NV classification adopts the ratio of a reliable fundamental frequency contour to the whole input interval. To evaluate the performance of our proposed method, we used 1360 voice commands and 736 noises in powered-wheelchair control in a real environment. These results indicate that the recall rate is $97.4 \%$ when the lowest threshold is selected for noise classification with precision 97.3\% in VAD. The proposed VAD, which rejects nonvoice input in preprocessing, can be helpful to realize a highly reliable system.

3pSP33. Estimation of a talker and listener's positions in a car using binaural signals. Madoka Takimoto (Grad. School of Information Sci., Nagoya Univ., Furo-cho, Chikusa-ku, Nagoya, Aichi, 464-8603 Japan, takimoto@sp.m.is.nagoya-u.ac.jp), Takanori Nishino, and Kazuya Takeda (Nagoya Univ., Japan)

The problem of estimating the location of a sound source is described which is based on signals observed at the entrances of the two ears. The purpose is to specify the talker's and listener's positions within a car using the binaural signal. The talker and the listener sit in two of the four car seats. In this experiment, two kinds of head and torso simulators are used as a talker and a listener. Given information includes the acoustic transfer functions for all positional patterns. Eight patterns of acoustic transfer functions are measured, involving those that have the same positional pattern, but in which the talker faces a different direction. A Gaussian mixture model for each positional pattern is generated. The parameters we used are interaural information such as the envelope of an interaural level difference. The models are evaluated by specifying the positional pattern. Results show that we can specify positions with up to $97 \%$ (35/36) accuracy using the binaural signals of two men. Then the input signal was expanded to one with background noise that resembles a real situation, and a model that involves motion of the talker's head was also considered.
3pSP34. An implementation of realtime speech speed control system using hierarchical cascaded integrator and comb filters. Hironobu Takei, Shogo Nakamura, Hiroto Saitoh, and I. Nyoman Sukadana (Tokyo Denki Univ., 2-1200 Muzai Gakuendai, Inzai Chiba, Japan, takei@sp.sie.dendai.ac.jp)

In this paper, our algorithm for speech speed control system and its DSP implementation are described. The system slows or speeds up speech without changing its pitch, which aids learning a foreign language, listening for older people, and so on. The proposed algorithm is based on the phase vocoder, which doesn't need extraction of voiced fragments from a speech signal. And the reconstruction of time-scaled signal involves no smoothing operation between voiced and unvoiced sections. The speech signal is decomposed to bandpassed frequency channels so that the envelope of each of the channels is stretched. Then the stretched envelope is multiplied by the corresponding sinusoidal, and by summing up the channel components the speed controlled signal is reconstructed. Each band has very narrow bandwidth, which requires a large amount of computation with a FIR-based design. To achieve realtime operation of our system, a hierarchical structured cascaded integrator and comb (HSCIC) filter is also introduced, which can have highly sharpened characteristics with only a few multipliers.

3pSP35. On the accuracy of speech source direction identification on the horizontal plane using 3-D audio displays. Hirotoshi Terada, Kazuhiro Kondo, and Kiyoshi Nakagawa (Yamagata Univ., Jounan 4-3-16, Yonazawa, 992-8510, Yamagata, Japan)

This paper describes initial results of speech source direction identification experiments on the horizontal plane. This is an initial study for the sound localization required in order to separate the individual speech source location to increase the intelligibility in remote conference systems. Our focus here was to determine the effect of sound source characteristics on the source direction identification accuracy. Sound sources were located on three target locations (forward, right, and left) and five (45 deg in both right and left added) on the horizontal plane using a real-time virtual acoustic environment rendering system (NASA SLAB). SLAB integrates HRTFs to render the source localization. The sound sources used were conversation speech, white noise, and conversation speech mixed with noise at an SNR of $0 \mathrm{~dB}$. The observers were asked to identify the direction of the rendered sound presented over a headphone. The results showed that the direction identification accuracy of conversational speech is approximately $20 \%$ higher than that of white noise in all directions tested. However, the accuracy of mixed conversation and white noise was about the same as the accuracy with white noise only, suggesting that the high-frequency components may be degrading the perceived accuracy.

3pSP36. Multipitch estimation based on correlation between a harmonic structure model and an observed spectrum for mixed audio signals. Yoichi Uchida and Naotoshi Osaka (Tokyo Denki Univ., School of Eng., 2-2 Kanda Nishiki-cho, Chiyoda-ku, Tokyo, 101-8457, Japan, uchida@srl.im.dendai.ac.jp)

In the research of sound source separation and resynthesis, multipitch estimation is an important topic. We propose a multipitch estimation technique for monaural audio signals. It introduces a method of calculating multiple pitches within a frame for a pitched sound signal. First, a harmonic structure is modeled for a pitch $M(\mathrm{~Hz})$, using the cases of odd integer multiples of $M$, even integer multiples of $M$, and odd integer multiples of $M / 2$. Then correlations are derived between each of the harmonic structure models and an observed spectrum. Harmonic structure models are expressed as the summation of Gaussian functions in the frequency domain, where each harmonic has a peak at its center frequency. An evaluation function is dynamically defined to test how well arbitrary values of $M$ fit the observed spectrum. The evaluation function is created by searching for weighted coefficients for the correlation values. Next, a number of maximal peaks equal to the number of sound sources are se- 
lected from the spectrum and defined as the estimation pitches. This method was applied to a mixed piano signal, and a mixed audio signal that includes flute, clarinet, and cello. The results are good except at octave unisons.

3pSP37. Transient detection algorithms for speech coding applications. Grzegorz Szwoch, Maciej Kulesza, and Andrzej Czyzewski (Gdansk Univ. of Technol., Narutowicza 11/12, 80-952 Gdansk, Poland)

One of the methods for improving transmitted speech quality is efficient encoding of transients. The majority of currently used speech codecs employ the parametric approach, thus they do not differentiate between transients and other signal components. A sine + noise + transients signal model, proposed earlier as a tool for wideband audio signal encoding, seems to be a promising solution for enhancing signal quality in speech coding. Methods of transient identifying for an efficient transform-domain encoding of transients has to be chosen carefully. Different transient detection algorithms are described in literature, most of them optimized for musical signals. The methods do not ensure efficient transient detection in speech signal, due to the different character of musical signals and speech. This paper provides a comparison of several transient detection methods, mostly based on evaluation of signal energy, that are now primarily used for musical signals. Their efficiency and application to transient detection in speech are evaluated and discussed. Differences between transient detection in wideband musical signals and speech are pointed out. Modifications to existing algorithms are proposed, allowing efficient transient detection and encoding in the proposed, wideband speech codec. [Research funded by the Polish Ministry of Education and Science within Grant No. 3 T11D 004 28.]

3pSP38. Sound source classification using temporal pattern for mixed sounds. Toru Taniguchi, Katsuhiko Shirai (Dept. of Comput. Sci., Waseda Univ., 3-4-1 Okubo, Shinjuku-ku, Tokyo, 169-8555 Japan, tani@shirai.cs.waseda.ac.jp), and Mikio Tohyama (Waseda Univ., Honjo-shi, Saitama 367-0035, Japan)

In speech information processing of real-world environments, how to deal with nonstationary acoustic noise such as background music or speech is a remaining problem while much progress has been made in the speech processing in stationary noise. Music or speech is a sound that has a complex spectral and temporal structure composed of many musical notes or phonemes, and this fact makes it difficult to estimate signals of music or speech separated from a foreground speech. For noise estimation of one-channel sound, only the prior knowledge of temporal or spectral structures of the noises is available besides the signal itself. Therefore, dealing with nonstationary noise of music or speech essentially requires the models representing the temporal and spectral structure of them. In our study, a classification method of nonstationary sounds using sinusoidal decomposition is proposed. In the method a classification of mixed sounds into sound categories, like speech, singing voices, or instruments, is performed based on temporal characteristics of spectral sequences analyzed by a spectral peak tracking algorithm. Temporal features are inspected by statistical analyses and discrimination experiments of sinusoidal sequences. The classification algorithm is evaluated by experiments of classification of mixed sound.

3pSP39. Multiple fundamental frequency estimation assisted by audio watermarking. Yi-Wen Liu (Boys Town Natl. Res. Hospital, 555 North 30th St., Omaha, NE 68131)

The success of computer segregation of sound sources from a singlechannel mixture often relies on the estimation of multiple fundamental frequencies. Instead of solving the problem directly, this presented work utilizes audio watermarks to assist sound source segregation. Here, individual sources are assumed to be available for watermark embedding be- fore mixing. Each source's short-time spectral peaks are aligned to frequency gridpoints labeled with a digital ID [Liu and Smith, "Audio Watermarking Based on Sinusoidal Analysis and Synthesis," International Symposium on Musical Acoustics, Nara, Japan (2004)]. A modified sound source is sinusoidally synthesized afterwards. In this sense, each source is embedded with a watermark. In an experiment, two sound sources are watermarked with two sets of gridpoints $P$ and $Q$, respectively. Points in $P$ and $Q$ interleave and are spaced 10 cents of a semitone apart. The watermarked sources are then linearly added. To demix them, sinusoidal trajectories found in the mixture are segregated based on whether its frequency more often aligns to $P$ or $Q$. Then, segregated signals are reconstructed by sinusoidal synthesis. Although the reconstructed signals do not sound like the original sources, the melodies of the original are successfully extracted from the reconstructed in monophonic pitch estimation.

3pSP40. Directions of arrival (DOA) estimation by particle filtering with dominant frequency components in noisy environments. Mitsunori Mizumachi, Norikazu Ikoma, and Katsuyuki Niyada (Kyushu Inst. of Technol., 1-1 Sensui-cho, Tobata-ku, Kitakyushu-shi, Fukuoka 804-8550, Japan)

In noisy environments, DOA estimation is useful for achieving attractive human-machine interfaces in the real world. This article proposes a robust DOA finder based on cross correlations between observed signals by particle filtering with frequency selectivity. The particle filter is flexible for modeling the system because it requires no linearity or Gaussianity on the model. The temporal trajectory of DOAs is modeled using a Markovian state space model. It is estimated through the state estimation procedure using particle filters. Markov modeling is expected to achieve stable DOA estimation in time. It is also a key for the proposed method to selectively use the dominant frequency components, such as formant frequencies of speech, to improve noise robustness. Assuming the quasistationarity of noise signals in the presence of a time-varying target speech signal, the dominant frequencies are extracted using a deterministic approach similarly to spectral subtraction. A set of the subband crosscorrelation functions at the dominant frequencies is regarded as likelihood and is used for updating particle weights to estimate posterior distribution. The feasibility of introducing both particle filtering and frequency selectivity into DOA estimation was confirmed in several noise conditions.

3pSP41. Azimuth and elevation estimation for multiple sound sources using two-dimensional frequency domain binaural model. Yoshifumi Chisaki, Sho Kawano, and Tsuyoshi Usagawa (Grad. School of Sci. and Technol., Kumamoto Univ., 2-39-1, Kurokami, Kumamoto, 860-8555 Japan, chisaki@cs.kumamoto-u.ac.jp)

Direction of arrival (DOA) estimation for multiple sound sources with only two sensors as the human being does is proposed. The frequency domain binaural model (FDBM) [Nakashima et al., Acoust. Sci. Technol. 24, 172-178 (2003)] was proposed as one binaural model. The method uses sound diffraction to estimate DOAs and segregate sound sources. The binaural model can estimate one-dimensional DOA, namely, azimuth. However, human beings can estimate not only azimuth of DOA but also elevation of that. In order to achieve an estimate of both azimuth and elevation simultaneously, a new DOA estimation algorithm based on interaural phase and level differences is proposed as a part of frequency domain binaural model. Results of DOA characteristics of the proposed binaural model estimation on multiple sound sources' condition is compared with that of human auditory characteristics. As a result, it was confirmed that performance on accuracy of DOA estimation depends on power level ratio among sound sources. [Research was partially supported by the Ministry of Education, Science, Sports and Culture, Grant-in-Aid for Scientific Research (C), 2006, 18500135 and carried out by the Cooperative Research Project Program of the RIEC, Tohoku University (Grant No. H16.A02).] 
3pSP42. Sound source localization using a distributed microphone system in real environments. Kook Cho, Hajime Okumura, Takanobu Nishiura, and Yoichi Yamashita (College of Information Sci. and Eng., Univ. of Ritsumeikan, 1-1-1, Noji-Higashi, Kusatsu-shi, Shiga 525-8577 Japan)

Accurate localization of sound sources is indispensable for highquality sound capture. The difference of distance from a sound source to two microphones causes a time delay of arrival (TDOA). The cross-power spectrum phase analysis (CSP) method has been proposed to estimate the TDOA based on correlation in signals. The sound source can be localized theoretically as a crossing point of sound directions estimated using two pairs of microphones. However, in a real environment, microphones receive sounds that have originated through reverberation and reflection. This situation makes TDOA estimation difficult, and accurate sound localization is difficult when using only two pairs of microphones. The authors propose a new method of sound localization based on multiple microphones that are widely distributed and placed under the ceiling of a room. A number of pairs of microphones give a series of CSP correlation coefficients as a function of the time delay. The CSP coefficients for hypothetical sound sources are accumulated over all the microphone pairs. The number of microphones is 16 . The sound source is localized as a hypothesis that maximizes the accumulated CSP coefficient. Experiment results obtained in a real room show that the proposed method improves the localization accuracy.

3pSP43. Free listening-point synthesizing method in a large microphone array using acoustic transfer function. Mehrdad Panahpour Tehrani, Yasushi Hirano, Shoji Kajita, Kenji Mase (Information Technol. Ctr., Nagoya Univ., Furo-cho, Chikusa-ku, Nagoya 464-8601, Japan), Takanori Nishino, Kazuya Takeda (Grad. School of Information Sci., Furo-cho, Chikusa-ku, Nagoya 464-8603, Japan), and Toshiaki Fujii (Grad. School of Eng., Furo-cho, Chikusa-ku, Nagoya 464-8603, Japan)

In this research a free listening-point synthesizing method is proposed by using the measured acoustic transfer function (ATF) in a large microphone array for future 3-D audiovisual systems. ATFs at the location of far-field microphones are estimated in frequency domain by using a recorded time-stretched pulse (TSP) signal broadcasted by speakers at the location of sources. Then an ATF pool is generated with a chosen density by synthesizing the estimated ATFs for each sound source. The ATF pool contains all the microphones' locations, plus synthesized ATFs with a chosen density from sound sources to any point on the arc which passes all of the microphones. The free listening-point is generated by selecting the ATF for each source from the ATF pool. Then the source microphone sounds are convolved with the chosen ATFs and added. The proposed method is evaluated by an array with 38 microphones in the far field on an arc with $r=4 \mathrm{~m}$ with $20 \mathrm{~cm}$ between each pair of microphones, and two microphones close to two sound sources with 1.5-m distance. Experimental results have been shown for the estimated ATFs and comparison of the synthesized arbitrary listening point with the actually recorded sound for a given point that shows satisfactory results.

3pSP44. On the numerical simulation of sound wave propagation using field programmable gate array device. Takao Tsuchiya (Dept. of Information Systems Design, Doshisha Univ., 1-3 Miyakodani, Tatara, Kyotanabe-shi, Kyoto 610-0321, Japan, ttsuchiy@mail.doshisha.ac.jp), Eiko Sugawara, and Yasusi Inoguchi (Japan Adv. Inst. of Sci. and Technol., 1-1 Asahidai, Nomi, Ishikawa 923-1292, Japan)

Numerical simulation of sound wave propagation using field programmable gate array (FPGA) device is proposed. The two-dimensional digital Huygensf model (DHM) or the transmission line matrix (TLM) model is configured in FPGA device as digital circuits. DHM is a numerical model in which the propagation and scattering of waves are simulated as the sequences of impulses scattering on the transmission line network as Huygens' principle states. The digital equivalent circuit can be easily devel- oped based on DHM because the formulation of DHM is very simple compared with the finite difference time domain (FDTD) method. In this paper, number of logic cells of the FPGA device required for a DHM element is estimated. To configure DHM elements in the FPGA device as much as possible, the fixed-point operations are applied to the DHM calculations. Numerical precision of DHM using the fixed-point operations is then discussed. The data length required for acoustic field simulation in practical precision is estimated. The capability of the FPGA simulator for room acoustics is discussed.

3pSP45. Signal processing for small-size microphone array systemApplication to teleconferencing system. Akira Gotoh, Yoshitaka Murayama, Jun Ota, Hareo Hamada (DiMAGIC, Sugishou Bldg 3F, 2-4-5 Kanda-Ogawamachi, Chiyodaku, Tokyo 101-0052, Japan), Yuko Watanabe, and Hareo Hamada (Tokyo Denki Univ., 2-1200 Muzaigakuendai, Inzai-shi, Chiba, Japan)

A small microphone array system, consisting of two or more closely arranged small microphones and a digital filter network, is newly proposed. A method based on sound field control using multiple-point leastsquares filter design contributed to the configuration of a smaller size microphone array system. Several directivity patterns having unique focus directions can be configured by changing each set of optimum filter coefficients. In this paper, a new method of scanning speaker direction using the small microphone array is proposed and several performances used in teleconferencing systems are evaluated.

3pSP46. Sensor signal processing circuit for echolocation using a delta-sigma modulated single-bit digital signal. Shinnosuke Hirata, Minoru K. Kurosawa (Tokyo Inst. of Technol., G2-32, 4259 Nagatsuta, Midori-ku, Yokohama, Japan, shinnosuke.hirata@ip.titech.ac.jp), and Takashi Katagiri (Sutekina Inc. Komagane, Japan)

Ultrasonic sensors are suitable for perception of the environment of mobile autonomous robots. Most bats use echolocation that is a highquality sensing system by their ultrasonic sensors [S. J. Gaioni, H. Riquimaroux, and N. Suga, "Biosonar behavior of mustached bats swung on a pendulum prior to cortical ablation," J. Neurophysiol. 64(6), 18011817 (1990)]. The echolocation can estimate the distance to the object, its relative velocity, and its scale. But, technological application of echolocation necessitates a large computational requirement because delay of the echo signal from the generated signal is estimated from cross correlation between the generated signal and the echo signal. A new sensor signal processing for echolocation is proposed by using a delta-sigma modulated single-bit digital signal. A delta-sigma modulated single-bit digital signal is a single-bit high-speed data stream. [S.R. Norsworthy, R. Schreier, and G. C. Temes, Delta-Sigma Data Converters (IEEE Press, New York, 1997] Using delta-sigma modulated single-bit digital signal processing, multiplication and accumulation of cross-correlation can be changed into logical operations: EXOR, NOT, SUM. Simulations show the possibility of estimation of the distance to the object from correlation of the echo signal with the generated signal converted single-bit data stream, along with reduced computational and circuit-scale requirements.

3pSP47. Modeling of phased antenna array of about 500 microphones, detecting landing airplanes. Gaetano Caronna, Ivan Roselli, and Pierluigi Testa (Dipartimento di Fisica Tecnica, Universit La Sapienza, Via Eudossiana, 18, 00100 Roma, Italy, gaetano.caronna@uniroma1.it)

The University La Sapienza participated recently in the project SAFEAIRPORT funded within the sixth framework program of the European Commission. This project aimed at developing a phased antenna array of about 500 microphones in order to acoustically detect and track flying airplanes. A preliminary field testing was performed at Rome-Fiumicino airport, however experimental data are not yet fully available. For this 
reason, a computer-aided model of the antenna phased array was developed and beam-forming was simulated with real input signals (acoustic emissions from a real landing aircraft with superimposed disturbing, spatially uncorrelated, pink noise of realistic level). Detection range was measured by applying classic shift and sum beamforming. An algorithm was tested in order to increase maximum detection range by exploiting information from more than one temporal frequency, assuming that disturbing environmental noise is uncorrelated on different temporal frequencies. Specific results are reported.

3pSP48. Compressive coding for multichannel audio signals using independent component analysis. Tadashi Mihashi, Tomoya Takatani, Shigeki Miyabe, Yoshimitsu Mori, Hiroshi Saruwatari, and Kiyohiro Shikano (Nara Inst. of Sci. and Technol., 8916-5 Takayama-cho, Ikoma-shi, Nara 630-0192, Japan)

This study addresses a novel compressive coding for multichannel audio signals with independent component analysis (ICA). In recent years, multichannel audio contents are widely spreading, and thus the transmission quantity is increasing. Consequently, audio-signal compression is a problem demanding prompt attention. Conventional compression methods utilizing sparseness assumption among audio-source signals have a problem that the method is likely to distort original sources owing to the lack of sparseness in the mixed signals consisting of two or more sound sources. To solve this problem, we propose an audio-signal compression strategy using ICA; ICA assumes that the source signals are mutually independent, and can estimate the source signals from the mixed signals without a priori information by applying an unmixing matrix. The decomposition of mixed signals leads to an enhancement of sparseness among signals. Furthermore, we propose a mechanism to detect time-frequency grids where sparseness is not satisfied, and for the detected grids we directly transmit multichannel signals to mitigate the distortion. The reconstruction process utilizes an inverse projection of the unmixing matrix to make correct lateralization of each sound source. Experimental result shows that the proposed method can compress mixed audio signals more efficiently and achieve a higher quality than the conventional method.
3pSP49. Novel interface using a microphone array for wheelchair control. Akira Sasou and Hiroaki Kojima (Natl. Inst. Adv. Industrial Sci. Technol. (AIST), Central\#2,1-1-1, Umezono, Tsukuba, Ibaraki, 305-8568 Japan, a-sasou@aist.go.jp)

This paper describes a novel interface that enables the control of a wheelchair by disabled people without having to use their hands. Thus far, a voice-driven wheelchair has been developed for disabled people who find it difficult to move their hands but are able to speak. Such wheelchairs usually adopt a headset microphone in order to reduce noise interference. However, a headset microphone is not practical because disabled people cannot wear the headset independently. Another problem faced is that the microphone shifts away from the mouth due to involuntary movements. In the proposed system, however, we use a microphone array that is mounted on a wheelchair, so that the disabled people do not need to wear the microphone. The system is also robust to noises and is able to recognize a face's direction by estimating the position of a sound source such as the mouth. For instance, when disabled people need to turn left, they face left and make a sound that is not restricted to voice commands. Thus, the proposed system does not require disabled people to utter clear voice commands. This feature enables the application of the system to more general cases.

3pSP50. Decomposition of the time reversal operator (DORT) for extended objects. Jean-Luc Robert (Philips Res. North America, 345 Scarborough Rd., Briarcliff Manor, NY 1510, jean-luc.robert@philips.com) and Mathias Fink (Universite Paris 7, 75005 Paris, France)

The decomposition of the time reversal operator, known by the French acronym DORT, is widely used to detect, locate, and focus on scatterers in various domains such as underwater acoustics, medical ultrasound, or nondestructive evaluation. In the case of point scatterers, the theory is well understood: the number of nonzero eigenvalues is equal to the number of scatterers, and the eigenvectors correspond to the scatterers' Green function. In the case of extended objects, however, the formalism is not as simple. We show that, in the Fresnel approximation, analytical solutions can be found, and that the solutions are functions called prolate spheroidal wavefunctions. These functions have been studied in optics and information theory as a basis of band-limited and time-limited signals. The theoretical solutions are compared to simulation results and previous experimental results Most importantly, we justify the intuition that for an extended object, the number of nonzero eigenvalues is proportional to the number of resolution cells in the object. The case of 3-D objects imaged by a 2-D array will also be described. 


\title{
Session 3pUW
}

\section{Underwater Acoustics: Sound Propagation: Measurements and Modeling II}

\author{
Nobuyuki Endoh, Cochair \\ Kanagawa Univ., Dept. of Engineering, 3-27-1 Rokkakubashi, Yokohama 221-8686, Japan \\ Martin Siderius, Cochair \\ Heat Light \& Sound Research Inc., 12730 High Bluff Dr., Ste. 130, San Diego, CA 92130
}

\section{Contributed Papers}

1:00

3pUW1. Measurements of temporal coherence of sound transmissions through shallow water. T. C. Yang (Naval Res. Lab., Washington, DC 20375)

Temporal coherence of acoustic signals propagating through fluctuating oceans has received much attention in the literature as the rate of signal coherence loss impacts the sonar performance. This paper presents the measurement results of temporal coherence of acoustic signals propagating through shallow water using data from three experiments in three different parts of the world. The data are broadband signals covering three frequency bands: low frequencies below $1.2 \mathrm{kHz}$, mid frequencies between 2 and $5 \mathrm{kHz}$, and high frequencies between 18 and $22 \mathrm{kHz}$. The source-receiver range covers 3,5,10, and $42 \mathrm{~km}$. Measurement issues and environmental impact on the temporal coherence are discussed. The signal coherence time, defined by the time that the signal becomes decorrelated, is deduced from the data. The signal coherence time varies with the source-receiver range and the signal frequency and is significantly influenced by the medium fluctuations. The data can be fit with a simple power law dependence on the range, frequency, and sound speed standard deviations. [This work is supported by the U.S. Office of Naval Research.]

\section{1:15}

3pUW2. Comparison of measured signal coherence time in shallow and deep water. T. C. Yang (Naval Res. Lab., Washington, DC 20375)

Temporal coherence of acoustic signals determines the processing of a sonar system for various applications. This paper studies the frequency and range dependence of the temporal coherence time of signal propagation in deep water versus that in shallow water. The signal coherence time has been measured in deep water since the 1970s, in terms of a parameter called the phase rate (the rate of phase change of the signal). Signal coherence time in shallow water has received little attention until only recently. We compare measurement data from more than a dozen of experiments covering a wide range of frequencies at different source-receiver ranges in deep and shallow water. We find that the signal coherence time in both shallow and deep water can be fitted with a universal equation, which exhibits a $\frac{3}{2}$ power frequency dependence and $\frac{1}{2}$ power range dependence. The signal coherence time in shallow water is two to five times longer than the signal coherence time in deep water at the same frequency and range. The signal coherence times are comparable between shallow and deep water if measured in terms of the range-to-depth ratio. [This work is supported by the U.S. Office of Naval Research.]

\section{1:30}

3pUW3. Spatial coherence of the acoustic field for propagation ranges 500-1000 $\mathbf{m}$ in an East China Sea channel. Peter H. Dahl and Jee Woong Choi (Appl. Phys. Lab., Univ. of Washington, Seattle)

Measurements of the vertical spatial coherence at ranges 500-1000 m and for transmission frequencies $2-20 \mathrm{kHz}$ were made as part of the Asian Sea International Acoustics Experiment (ASIAEX) conducted in the East
China Sea (depth $105 \mathrm{~m})$. In an earlier work [J. Acoust. Soc. Am. 115, 589-599 (2004)], ASIAEX spatial coherence measurements corresponding to arrivals interacting once with the sea surface were modeled using the van Cittert-Zernike theorem in conjunction with model estimates of bistatic scattering scattering from the sea surface. The theorem relates the spatial coherence of an observed field to the distant source intensity distribution associated with this field. Here, the approach from the 2004 work is extended to study the full multiple path arrival involving bistatic scattering from the sea surface, and coherent reflection plus bistatic scattering from the seabed. Model and data comparisons are made for the vertical direction and predications are made for coherence in horizontal, and horizontal-transverse direction.[Work supported by the ONR.]

\section{$1: 45$}

3pUW4. Statistics of the Parseval sum, match filter, and instantaneous single-frequency transmissions in an ocean waveguide. Mark Andrews (Northeastern Univ., 409 Dana Res. Ctr., Boston, MA 02115-5000), Tianrun Chen, Sunwoong Lee, Nicholas Makris (MIT, Cambridge, MA 02139), and Purnima Ratilal (Northeastern Univ., Boston, MA 02115-5000)

During the ONR Main Acoustic Clutter Experiment of 2003 on the New Jersey Continental Shelf, short duration broadband pulses with 50- to $150-\mathrm{Hz}$ bandwidths were transmitted at $50-\mathrm{s}$ intervals from a moored source array in the $300-$ to $1500-\mathrm{Hz}$ range. The signals were measured by a towed horizontal receiving array at varying ranges from the source. The broadband data are processed in a variety of ways including the use of Parseval sum and match-filter, and as an instantaneous single frequency transmission. The ping-to-ping fluctuations in the measured acoustic data are a result of changes in the waveguide modal intereference structure due to both motion of the array and presence of time-dependent random internal waves. Our analysis shows that the Parseval sum and match-filter outputs for the direct arrival have significantly smaller standard deviations compared to the instantaneous single frequency transmission. We account for these observations by modeling the broadband acoustic field intensity in both a static waveguide and with Monte Carlo simulations in a fluctuating waveguide, as well as with the use of statistical distributions for the broadband acoustic field. We present an approach for empirically quantifying the range-dependent degradation in the match-filter output in a multimodal waveguide.

\section{2:00}

3pUW5. Estimation for velocity dispersion of normal modes in a laboratory waveguide. Cao Zhengliang, Wang Suping, and Zhou Shihong (Hangzhou Appl. Acoust. Res. Inst., Hangzhou 310012 China)

As one character of normal modes, velocity dispersion defined as velocity dependent on frequency is related to the environment of a fluid and seabed in a shallow water waveguide. It is important to inverse geoacoustic parameters or analyze normal mode propagation in the waveguide. With waveforms measured by a line array in a laboratory waveguide, velocity dispersion of normal modes is estimated by Prony's 
method. Because of the influence of the sum of many modes in a frequency, the estimated dispersions are disordered and discontinued. While a number of modes fewer than that, in fact, are previously selected, the results can be improved on velocity dispersion curves. On the other hand, clear curves can be obtained for the components of waveforms intercepted by an adaptive time window. These results are consistent with that of synthetic waveforms simulated by a normal mode program. Comparing the dispersions estimated from data in different depths, there are abundant high-order modes in this case. [Work supported by SKLF.]

\section{2:15}

3pUW6. PlaneRay: An underwater acoustic propagation model using ray tracing and plane wave reflection coefficients. Jens M. Hovem and Hefeng Dong (Dept. of Electron. and Telecommunications, Norwegian Univ. of Sci. and Technol., No. 7491, Trondheim, Norway)

Recently there has been a renewed interest in using ray tracing techniques for propagation modeling in underwater acoustics and this paper describes a newly developed model. A special and essential feature of this model is a unique sorting and interpolation routine for efficient determination of a large number of eigenrays and also for range-dependent environments. No rays are traced into the bottom and the bottom interaction is modeled by plane wave reflection coefficients. In the current version of the model the bottom structure consists of a fluid sediment layer over a solid half-space. Important considerations are the accuracy of the ray model and, in particular, how accurate the plane wave reflection coefficients can represent the effects of a layered bottom. This problem is analyzed by comparing the time and frequency domains solution of the ray model with models based on the wave number integration technique. There are two conflicting requirements since ray tracing is valid for high frequencies and the used plane wave reflection coefficient is valid for low frequencies where the sediment layers are thin compared with the acoustic wavelength. A number of illustrative examples are presented.

\section{2:30}

3pUW7. Ray-based acoustic localization of cavitation in a highly reverberant environment. Natasha A. Chang and David R. Dowling (Dept. of Mech. Eng., Univ. of Michigan, 1231 Beal Rd., 2019 Lay Autolab, Ann Arbor, MI 48109)

Acoustic localization techniques are applied in a water tunnel to study low event rate cavitation in a vortical flow. The measured center frequency and bandwidth of the acoustic pulse from the collapse of a small isolated cavitation bubble are $10 \mathrm{kHz}$ and $200 \mathrm{kHz}$, respectively, and the measured pulse duration is $\sim 15-20 \mu \mathrm{s}$. Its timing and exact waveform is not known. An array of 16 receiving hydrophones is used to estimate the source location in the test section of the water tunnel. The direct path signal used for this effort can be buried in background hydrodynamic noise from the water tunnel. Fortunately some of the direct-path signal arrival is distinct enough for acoustic localization. The signal coda is not used in the present effort. The received direct-path signals are crosscorrelated between hydrophones to obtain arrival time differences. These arrival time differences are used in conjunction with a simple ray-based acoustic model to estimate the source location in three dimensions. Once the cavitation bubble is located, the signal can be back propagated and part of the free space signal estimated and correlated with high-speed video of cavitation bubble dynamics. [Work sponsored by ONR codes 333 and 321.]

\section{2:45}

3pUW8. Bistatic invariance for active sonar systems. Lisa Zurk, Jorge Quijano, Manish Velankar (Portland State Univ., P.O. Box 751-ECE, Portland, OR 97207, zurkl@cecs.pdx.edu), and Dan Rouseff (Univ. of Washington, Seattle, WA 98105)

The concept of an invariant time-frequency relationship for passive acoustic intensity has been established through the consideration of interfering normal modes traveling in a shallow water channel. This formulation has received considerable attention in the past and has been applied to the interpretation of passive lofargrams. For bistatic active geometries, the acoustic energy propagates along two distinct pathways: source-to-target and target-to-receiver. In recent work we proposed that this more complicated propagation may also exhibit an invariant structure, thus suggesting potential for improved target tracking through the application of invariantbased track constraints. In this work, further exploration of this phenomenon is presented, with specific attention to the impact of the target scattering matrix that couples energy at the target between incoming and outgoing modes. Simulation results are presented for a variety of underwater environments to examine the effect on the invariant structure. Results are also presented and discussed from spectrograms produced from active sonar data obtained in shallow water environments.

\section{3:00-3:15 Break}

\section{$3: 15$}

3pUW9. Time-frequency analysis of mode coupling in shallow-water sound propagation. Mohsen Badiey (Ocean Acoust. Lab., Univ. of Delaware, Newark, DE 19716), Valery Grigorev, Boris Katsnelson (Voronezh Univ., Voronezh 394006 Russia), and James Lynch (WHOI, Woods Hole, MA 02543)

Time-frequency (TF) analysis for propagation of broadband sound pulses in shallow water in presence of perturbations of waveguides parameters, such as internal waves, temperature fronts, or bottom variability is considered. Due to waveguide dispersion one can construct a TF diagram for individual normal modes using Gabor transform for each received pulse. If we have a temporal sequence of propagating pulses then we can find a temporal sequence of TF diagrams. It is shown that in the presence of spatial and temporal waveguide perturbations (such as the sound-speed profile in the water column or the sea bottom) structure of a TF diagram and its temporal behavior depends on waveguide parameters, on the source-receiver positions, and on the character of mechanisms influencing the sound propagation, such as mode coupling, horizontal refraction, or adiabatic regime. Dependence of the TF diagram on waveguide variability in geotime provides information about temporal behavior of the perturbations. Structure of TF diagram is analyzed for a shallow-water waveguide model as well as for the SWARM95 experimental data. [Work supported by ONR and RFBR.]

\section{$3: 30$}

3pUW10. Propagation of impulsive broadband signals in a coastal ocean setting during the 1996 Shelfbreak Primer experiment. Steven E. Crocker (Naval Undersea Warfare Ctr., Newport, RI 02841), James H. Miller, Gopu R. Potty, Georges A. Dossot (Univ. of Rhode Island, Narragansett, RI 02882), and James F. Lynch (Woods Hole Oceanogr. Inst., Woods Hole, MA 02543)

The 1996 Shelfbreak PRIMER experiment provided an opportunity to study the impulse response of a coastal ocean waveguide using broadband acoustic signals from explosive sources. Acoustic transmission paths in the vicinity of the shelfbreak included various orientations ranging from upslope to along-slope, including interactions with a shelfbreak front. Acoustic receptions were acquired with a 16-element vertical line array (VLA). In addition, thermistor string data acquired at the receiving array suggest that a group of high-amplitude, internal waves traversed the test site during this portion of the experiment. Modal decomposition of broadband signal arrivals was performed to investigate variation of the impulse response during source deployments. Variations in the received spectra of individual normal modes were compared with numerical predictions for impulsive broadband signals propagating in range-dependent environments constructed using oceanographic data available for the test site. The potential for mode coupling and three-dimensional effects to shape the modal and spectral distribution of received energy is being investigated. Numerical and experimental results will be discussed. [Work supported by Office of Naval Research.] 
3pUW11. Underwater propagation at $16 \mathrm{~Hz}$ from French Polynesia to New Zealand: Echoes from islands, banks, and seamounts, and attenuation by seamounts. Marshall V. Hall (9 Moya Crescent, Kingsgrove, New South Wales 2208, Australia, marshallhall@optushome.com.au)

Underwater signals from two nuclear shots fired in Fangataufa and three in Mururoa during 1995/96 were recorded off northern New Zealand, a geoid distance of $4660 \mathrm{~km}$. The signals have been bandpass filtered at 16 $\mathrm{Hz}$ and found to contain up to six echoes. The causative scattering seafloor features were estimated from the travel times. For some echoes there was ambiguity in the feature and hence some uncertainty in the deflection angle (DA). Amongst the six echoes, the DA ranged between 20 and 56 deg. In the two Fangataufa signals, echo intensities relative to the intensity of the direct pulse ranged between -44 and $-25 \mathrm{~dB}$, and this relative intensity (RI) decreased with increasing DA. The highest feature on the direct path from Fangataufa, a seamount whose peak is $1.6 \mathrm{~km}$ deep, appears to have had little effect on the signal. In the three Mururoa signals, there were fewer echoes, and RI did not vary significantly with DA. The highest feature on the direct path from Mururoa was a seamount whose peak is $0.7 \mathrm{~km}$ deep. This seamount attenuated the direct signal at New Zealand by approximately $23 \mathrm{~dB}$, as well as paths to features at small angles from the direct.

\section{4:00}

3pUW12. Features of the broadband acoustic propagation in very shallow water. Seongwook Lee, Kyu-Chil Park, Jong Rak Yoon (Div. of Electron., Comput. and Telecommunication Eng., Pukyong Natl. Univ., 599-1 Daeyon-3dong, Namgu, Pusan 608-737, Korea), and Phil-Ho Lee (Agency for Defense Development, Jinhae, Gyungnam 645-600, Korea)

In shallow water, acoustic propagation is severely influenced by the property and structure of the bottom. We present the results of acoustic measurement conducted in a very shallow water of about $11-\mathrm{m}$ depth to characterize the features of broadband acoustic propagation. In the measurements using both of the ship and projectors as sources, the signal was received at four hydrophones, two of them were located in the water and another two were located in the sediment. Broadband propagation losses derived from ship noise revealed the frequency band that has high losses around $120-140 \mathrm{~Hz}$. Analysis results on the possible causes of high losses at that frequency band are presented.

\section{$4: 15$}

3pUW13. A stochastic model for underwater acoustic channel impulse function in the Kauai experiment. Aijun Song, Jing Luo, and Mohsen Badiey (College of Marine Studies, Univ. of Delaware, Newark, DE 19716)

Modeling of underwater acoustic channel impulse function has been of interest to the research community. As is well known, the underwater acoustic wave propagation depends on a number of environmental parameters, such as the sound speed profile along the propagation track, sea surface condition, and source/receiver geometry. The fluctuations of these environmental parameters cause the variability of the acoustic wave propagation in the shallow water region. In this paper, a stochastic model for the channel impulse function is proposed. The characteristics of the each eigenray, such as arrival time, average intensity, and coherence time, are extracted from the Kauai experiment (2003). The correlation between the model parameters and the environmental variability will also be shown.
3pUW14. Measurements of 3-D propagation effects at a shelfbreak front. Kristy A. Moore, James H. Miller, Gopu R. Potty, Georges A. Dossot (Dept of Ocean Eng., Univ. of Rhode Island, 217 Sheet Bldg., Narragansett, RI 02882), Scott M. Glenn ((Rutgers Univ., NB, NJ 08901), and James F. Lynch (Woods Hole Oceanogr. Inst., Woods Hole, MA 02543)

Acoustic data collected on two Webb Slocum gliders deployed by Rutgers University during the Shallow Water Experiment (SW06) on the continental shelf off New Jersey are presented. Acoustic recording systems were attached to the two gliders, which also collected oceanographic data. These gliders periodically surfaced for GPS fixes and data transfer via satellite phone. A major goal of these measurements was to quantify 3-D propagation effects including the horizontal analog to the classical Lloyd's mirror. The Lloyd's mirror effect is produced by the fact that fronts can totally internally reflect sound incident upon them at low grazing angles. The direct and reflected arrivals can add up to produce an interference pattern in range, potentially increasing the received intensity level by $6 \mathrm{~dB}$ [Lynch et al., IEEE J. Ocean. Eng., in press]. The gliders crossed the shelfbreak front at ranges of approximately $20 \mathrm{~km}$ from the sources and in water depths of approximately $80 \mathrm{~m}$. Measurements of the acoustic arrivals from 224 and $400 \mathrm{~Hz}$ tomography sources are compared to 3D modeling results. The increase in the intensity level predicted by the horizontal ray-vertical mode model is discussed. [Work supported by Office of Naval Research.]

\section{$4: 45$}

3pUW15. Arrival time change of long distance sound travel in the central Pacific. Hanako Ogasawara, Toshiaki Nakamura (Dept. of Earth and Ocean Sci., Natl. Defense Acad., 1-10-20 Hashirimizu, Yokosuka, Kanagawa 239-8686, Japan), Hiroyuki Hachiya (Chiba Univ., Inage-ku, Chiba 269-8522, Japan), Hidetoshi Fujimori (Japan Agency for Marine-Earth Sci. and Technol., Yokosuka, Kanagawa 237-0061, Japan), and Koichi Mizutani (Univ. of Tsukuba, Tsukuba, Ibaraki 205-8573, Japan)

Ocean acoustic tomography (OAT) is a valuable method for observations of oceanographic phenomena over wide areas. A tomography experiment using seven $200-\mathrm{Hz}$ transceivers at depths around $1000 \mathrm{~m}$ was executed in the central equatorial Pacific Ocean during January through December 2000 to monitor ocean phenomena related to El Niño and southern oscillation (ENSO). Because sound that propagates in the ocean takes various paths depending on its incident angle, the sound arrival times showed time lags. Arrival times of the first arrival signals were used to evaluate fluctuations throughout the year. Positional data of transceivers calculated from transponders indicated that major fluctuations of arrival times resulted from location movements of transceivers. The arrival times were corrected by calculating the distance using the positional data to remove distance effects in each transmission. Tidal effects were observed from spectral analyses of the corrected arrival times throughout the experimental period. Reciprocal arrival time differences between one pair of transceivers showed different changes during mid-September through November in comparison to other reciprocal arrival times of several paths. This change might have resulted from some kind of oceanic variation. Acoustic observations are advantageous for detecting various changes over long periods. 